\title{
Neutrino oscillation phenomenology in the standard model and beyond
}

\author{
Tesi Doctoral \\ Programa de Doctorat en Física \\ Christoph Andreas Ternes \\ Directora: Maria Amparo Tórtola Baixauli

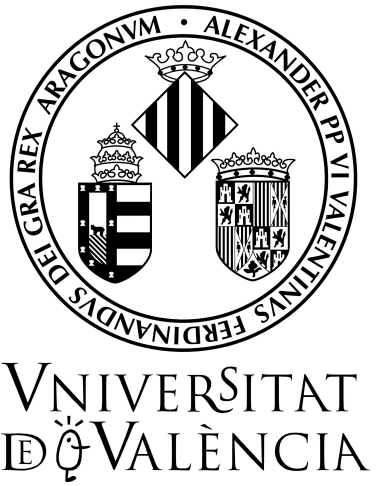

IFIC (CSIC - Universitat de València)

Departament de Física Teòrica

Març 2020 

A mi familia y mis amigos no físicos.

Por siempre apoyarme sin tener idea de lo que hacía. 

María Amparo Tórtola Baixauli, investigadora Ramón y Cajal de la Universitat de València,

\section{CERTIFICA:}

Que la presente memoria "Neutrino oscillation phenomenology in the standard model and beyond" ha sido realizada bajo su dirección en el Institut de Física Corpuscular, centro mixto de la Universitat de València y del CSIC, por Christoph Andreas Ternes y constituye su Tesis para optar al grado de Doctor en Física.

Y para que así conste, en cumplimiento de la legislación vigente, presenta en el Departament de Física Teòrica de la Universitat de València la referida Tesis Doctoral, y firma el presente certificado.

Valencia, a 24 de marzo de 2020.

María Amparo Tórtola Baixauli 


\section{List of publications}

The thesis is based on the following scientific publications :

1. Status of neutrino oscillations 2018: $3 \sigma$ hint for normal mass ordering and improved CP sensitivity

P.F. de Salas, D.V. Ferero, C.A. Ternes, M. Tórtola, J.W.F. Valle

Physics Letters B (2018), Volume 782, arXiv:1708.01186

and its updated preliminary version

2. Neutrinos, DUNE and the world best bound on CPT invariance

G. Barenboim, C.A. Ternes, M. Tórtola

Physics Letters B (2018), Volume 780, arXiv:1712.01714

3. New physics vs new paradigms: distinguishing CPT violation from NSI G. Barenboim, C.A. Ternes, M. Tórtola

The European Physical Journal C (2019), Volume 79, Number 5, arXiv:1804.05842

4. Constraining the invisible neutrino decay with KM3NeT-ORCA

P.F. de Salas, S. Pastor, C.A. Ternes, T. Thakore, M. Tórtola

Physics Letters B (2019), Volume 789, arXiv:1810.10916

5. Neutrino oscillation probabilities through the looking glass

G. Barenboim, P.B. Denton, S.J. Parke, C.A. Ternes

Physics Letters B (2019), Volume 791, arXiv:1902.00517

6. Neutrino mass ordering at DUNE: an extra $\nu$ bonus

C.A. Ternes, S. Gariazzo, R. Hajjar, O. Mena, M. Sorel, M. Tórtola

Physical Review D (2019), Volume 100, Issue 9, arXiv:1905.03589

7. Quasi-Dirac neutrino oscillations at DUNE and JUNO

G. Anamiati, V. De Romeri, M. Hirsch, C.A. Ternes, M. Tórtola

Physical Review D (2019), Volume 100, Issue 3, arXiv:1907.00980

Other publications, related to the topic of the thesis, but not discussed:

1. Testing a lepton quarticity flavor theory of neutrino oscillations with the DUNE experiment

R. Srivastava, C.A. Ternes, M. Tórtola, J.W.F. Valle

Physics Letters B (2018), Volume 778, arXiv:1711.10318 
2. Neutrino masses and their ordering: Global Data, Priors and Models

S. Gariazzo, M. Archidiacono, P.F. de Salas, O. Mena, C.A. Ternes, M. Tórtola

Journal of Cosmology and Astroparticle Physics (2018), Volume 2018, March 2018, arXiv:1801.04946

3. Zooming in on neutrino oscillations with DUNE R. Srivastava, C.A. Ternes, M. Tórtola, J.W.F. Valle

Physical Review D (2018), Volume 97, Issue 9, arXiv:1803.10247

4. Exploring the intrinsic Lorentz-violating parameters at DUNE

G. Barenboim, M. Masud, C.A. Ternes, M. Tórtola

Physics Letters B (2019), Volume 788, arXiv:1805.11094

5. Neutrino Mass Ordering from Oscillations and Beyond: 2018 Status and Future Prospects

P.F. de Salas, S. Gariazzo, O. Mena, C.A. Ternes, M. Tórtola

Frontiers in Astronomy and Space Sciences, Issue 5 (2018), arXiv:1806.11051

6. Sterile neutrinos with altered dispersion relations revisited G. Barenboim, P. Martínez-Miravé, C.A. Ternes, M. Tórtola

Journal of High Energy Physics 2020, 70 (2020), arXiv:1911.02329

7. Probing neutrino quantum decoherence at reactor experiments

A. de Gouvêa, V. De Romeri, C.A. Ternes arXiv:2005.03022

8. $C P T$ and $C P$, an entangled couple G. Barenboim, C.A. Ternes, M. Tórtola arXiv:2005.05975 



\section{Contents}

$\begin{array}{ll}\text { Introduction } & 1\end{array}$

I The present 5

$\begin{array}{lll}1 & \text { Neutrino oscillations } & 7\end{array}$

1.1 Neutrino oscillations in vacuum . . . . . . . . . . . . . . . 7

1.2 Neutrino oscillations in matter . . . . . . . . . . . . . . 11

1.3 Neutrino oscillations in constant matter . . . . . . . . . . . . . . . 14

1.3.1 Expansions under consideration . . . . . . . . . . . . . 15

1.3.2 Comparison of expansions . . . . . . . . . . . . . . . 29

1.4 Final remarks . . . . . . . . . . . . . . . . . . . . 36

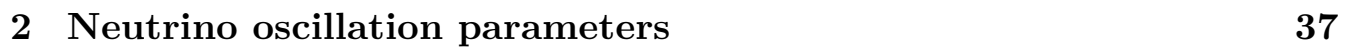

2.1 Experiments included in the global fit . . . . . . . . . . . . . . 39

$2.1 .1 \quad$ Solar experiments and KamLAND . . . . . . . . . . . . . 40

2.1 .2 Short-baseline reactor experiments . . . . . . . . . . . . 43

2.1 .3 Atmospheric experiments . . . . . . . . . . . . . . 46

2.1 .4 Accelerator experiments . . . . . . . . . . . . . . . . . . 49

2.2 Global fit results . . . . . . . . . . . . . . . . . . . 51

2.3 Summary and discussion $\ldots \ldots \ldots \ldots \ldots$

\begin{tabular}{|lll}
3 & Global fit to oscillation data without CPT invariance & 63
\end{tabular}

3.1 Introduction . . . . . . . . . . . . . . . . . 63

3.2 Global fit to $\nu$ and $\bar{\nu}$ oscillation data . . . . . . . . . . . . 67

3.3 Current bounds on CPT violation in the neutrino sector . . . . . . 68 
\begin{tabular}{ll}
\hline II The future & 71
\end{tabular}

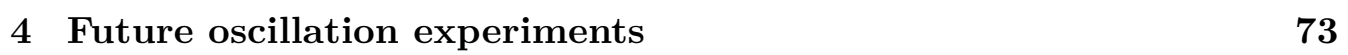

\begin{tabular}{|lll}
5 & Standard neutrino oscillations at DUNE & 81
\end{tabular}

5.1 Simulation of the DUNE experiment . . . . . . . . . . . . . . 81

5.2 Beam determination of oscillation parameters . . . . . . . . . . 82

5.3 Mass ordering determination with atmospheric neutrinos . . . . . . 83

5.3 .1 Matter effects and atmospheric neutrinos $\ldots \ldots$. . . . 85

5.3 .2 Atmospheric neutrino events in DUNE: muon capture in

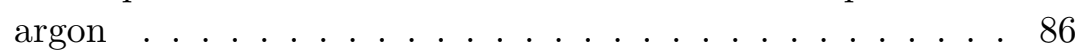

$5.3 .3 \quad$ Analyses and Results . . . . . . . . . . . . . . . . . . 94

$\begin{array}{lll}6 & \text { CPT violation and DUNE } & 99\end{array}$

6.1 Improving the current bounds on CPT violation at DUNE . . . . . 99

6.1 .1 Simulation of the DUNE experiment . . . . . . . . . . 99

6.1.2 DUNE sensitivity to CPT-violating neutrino oscillation pa-

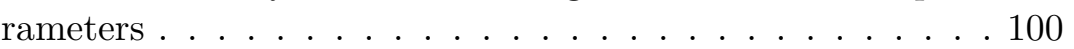

6.1 .3 Obtaining imposter solutions . . . . . . . . . . . . 103

6.1.4 Probing the T2K neutrino and antineutrino analysis in DUNE106

6.2 Robustness of the CPT results: CPT versus NSI . . . . . . . . 107

6.2 .1 Theoretical background . . . . . . . . . . . . . 110

$6.2 .2 \quad$ Analytical results at the probability level $\ldots . . . . . .111$

6.2 .3 Results from the simulation of DUNE . . . . . . . . . . . . 114

\begin{tabular}{|lll}
7 & Quasi-Dirac neutrino oscillations at DUNE and JUNO & 119
\end{tabular}

7.1 Introduction . . . . . . . . . . . . . . . 119

7.2 Quasi-Dirac neutrino oscillations . . . . . . . . . . . . . . 120

7.3 Simulation of experiments $\ldots \ldots \ldots$. . . . . . . . . . . . 126

7.4 Results and discussion . . . . . . . . . . . . . . . . . . . . . 128

8 Long live the neutrino 135

8.1 Introduction . . . . . . . . . . . . . . . . . . . . . . . . 135

8.2 Invisible neutrino decay $\ldots \ldots \ldots$. . . . . . . . . . . 136

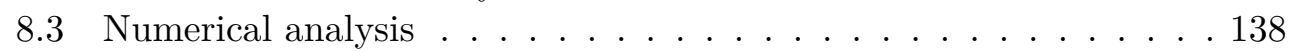

8.3 .1 Simulation of the neutrino signal in ORCA . . . . . . . . 140

8.3 .2 The role of systematic uncertainties . . . . . . . . . . 143

8.4 Results and discussion . . . . . . . . . . . . . . . . . . . . . . 144

8.4.1 Estimated sensitivity to the invisible decay . . . . . . . . 145 
$8.4 .2 \quad$ Effect of decay on standard oscillation parameters . . . . . 147

$8.4 .3 \quad$ Other future prospects for invisible neutrino decay . . . . . 148

Conclusions

151

\begin{tabular}{|l|l|}
\hline A Neutrino oscillation parameters 2017 & 155
\end{tabular}

A.1 Data included in the $2017 \mathrm{fit}$. . . . . . . . . . . . . . . . . 155

A.2 Global fit 2017 . . . . . . . . . . . . . . . . . . . . . 157

B Neutrino oscillation parameters 2020 161

B.1 Data to be included in the $2020 \mathrm{fit}$. . . . . . . . . . . . . . . 161

Statement of contributions 165

\begin{tabular}{ll}
\hline Resumen de la Tesis & 167
\end{tabular}

\begin{tabular}{ll}
\hline References & 183
\end{tabular} 



\section{Introduction}

The principal topic of this thesis is the phenomenology of neutrino physics. It is currently one of the most active research fields interconnecting particle physics, astrophysics and cosmology. Here, the focus is set on the phenomenology of neutrino oscillations, which are a result of the quantum superposition of neutrino mass eigenstates and neutrino mixing. In order to be able to oscillate, at least two neutrinos need to be massive. Therefore, the observation of neutrino oscillations confirms that neutrinos are massive particles, resulting, together with the overwhelming evidence for the existence of dark matter from cosmological observations, in our first evidence for physics beyond the Standard Model (SM) of particle physics. However, the neutrino mass-mechanism is unknown, although several models explaining neutrino masses have been suggested. In these models, testable at current and future experiments, the particle content has to be extended with respect to the SM and also new interactions can be present. Therefore, the "easiest" manifestation of neutrino oscillations, the so-called 3-neutrino paradigm, might be incomplete. Current experiments are, unfortunately, not sensitive enough to observe different, sub-leading effects in neutrino oscillations. However, future experiments might be able to detect these effects. During this $\mathrm{PhD}$, several projects related to neutrino oscillations, in the standard 3-neutrino paradigm and beyond, have been studied, but not all the results will be covered in this thesis. Here, we simultaneously give a general introduction to the topic of the thesis and review the results obtained in this period. This $\mathrm{PhD}$ thesis is divided in two parts: the first one deals with the current status of neutrino oscillations, while in the second part we discuss the discovery potential at future experiments, where a special emphasis is set on the DUNE experiment.

In the first part, we start in Chapter 1 with a general overview on neutrino oscillations and how the neutrino oscillation probability can be calculated. We will see that, when neutrinos travel through matter, their flavor oscillations are altered through the interactions with electrons in the medium. In this case, it is difficult to derive exact formulas for the oscillation probabilities. However, many 
approximate formulas exist. In the main part of Chapter 1 we compare these approximations in terms of their usefulness [1. Next, we discuss how to extract the parameters describing neutrino oscillations from experimental data. This is done by performing a global fit to neutrino oscillation data [2], as done in Chapter 2 . When combining these results with observations from cosmology, additional information on the neutrino mass ordering can be obtained. This has been done in Refs. [3] and 4, but will not be discussed further in this thesis. Using oscillation data one can bound CPT invariance by analyzing neutrino and antineutrino data separately. This was performed in Chapter 3 , where we give some more details than in Ref. [5]. This is the first departure from the SM discussed in this thesis, since in the SM neutrinos and antineutrinos should oscillate the same way apart from the $\mathrm{CP}$-violating effects present in the SM! Another beyond the standard model (BSM) scenario analyzed during this thesis is the possible existence of an eV-scale "sterile" neutrino. A global fit to neutrino oscillation data in this so-called $3+1$ picture is currently being performed [7]. Although there exist anomalous results which could indicate the existence of a fourth neutrino in several data sets, large tensions between different data arise in the global analysis. Since there are no final results available yet, we will not discuss this topic further here. Several scenarios have been then proposed to ease these tensions. One of these proposals is considering several new sterile neutrinos with altered dispersion relations. This way, a resonant behavior in the oscillation probability arises in the energy range relevant for experiments with results in favor of sterile neutrinos, while for higher energies where no evidences for new states have been found, the standard 3-neutrino paradigm is recovered. Although promising, we have shown that a scenario like this is excluded by current oscillation data 8]. Since these results are closely related to sterile neutrinos we do not discuss them here either 2 .

In the second part of the thesis, we discuss the capabilities of future neutrino oscillation experiments. We start with a general overview of the future facilities in Chapter 4. In the first part of Chapter 5 we describe the simulation of DUNE and how well it will improve the measurement of several neutrino oscillation parameters. In the second part of this chapter, we focus on the possibility to extract the neutrino mass ordering using atmospheric neutrinos at DUNE 10 where we show how the use of muon capture in argon can improve this measurement. Note that the neutrino mixing pattern could be due to some underlying flavor symme-

\footnotetext{
${ }^{1}$ During the evaluation process of the thesis, we finished another work 6 related to $\mathrm{CP}$ and CPT violation which will not be discussed here.

${ }^{2}$ Another project, related to coherence loss in propagation of reactor antineutrinos, was finished while the thesis has been evaluated 9]. This topic will not be discussed here.
} 
try. In this case, the neutrino oscillation parameters could be predicted from the intrinsic parameters of the model. Flavor models predicting a vanishing reactor mixing angle, for example, are already ruled out by neutrino oscillation experiments. Most of the models predicting atmospheric mixing and $\mathrm{CP}$ violation, however, remain allowed. Since DUNE will measure these neutrino oscillation parameters with extremely good precision, this question could be addressed in the near future. Several different scenarios of this type have been analyzed in Refs. [11] and [12], but are not going to be discussed in this thesis.

In Chapter 6, we resume the discussion of Chapter 3 in the context of DUNE. We show how the current limits on CPT invariance violation could be improved by DUNE. We also discuss a method to distinguish a possible signal of CPT violation from general neutrino non-standard interactions [13. The results discussed here are model-independent, since they do not depend on a theory of CPT violation; our only assumption is that neutrinos and antineutrinos oscillate with different oscillation parameters. However, in Ref. [14 we also discussed CPT and Lorentz violation in the context of the so-called standard model extension (SME), a model which extends the SM by including all renormalizable CPT and Lorentz-violating operators.

Some models of neutrino masses contain six neutrinos ( 3 active and 3 sterile), with very small Majorana mass terms. This results in active-sterile neutrino pairs with very small mass splittings among them. They are referred to as quasi-Dirac neutrinos and they would alter the standard 3-neutrino oscillation pattern. In Chapter 7, we discuss the implications of such neutrinos on DUNE and also on the future reactor experiment JUNO [15]. Finally, in Chapter 8 we turn our attention to atmospheric neutrinos at the next generation experiment ORCA. There, we study the sensitivity of the experiment to the invisible neutrino decay [16], which can be present, for example, in Majoron models, relating neutrino physics with the physics of dark matter. We will close with a few final remarks. 



\section{Part I}

\section{The present}





\section{Chapter 1}

\section{Neutrino oscillations}

Neutrino oscillations are a quantum mechanical phenomenon analogous to the oscillation of kaons $K^{0}-\bar{K}^{0}$ introduced in the 1950s by Pontecorvo 17.18. Originally, they were introduced as oscillations between neutrinos and antineutrinos, because at this time only the electron neutrino had been observed. However, soon later the muon neutrino was discovered and Pontecorvo's idea was adapted to oscillations between electron and muon neutrinos 19. Even before the discovery of the tau neutrino, physicists were convinced that a third neutrino existed. In 1962, Maki, Nakagawa and Sakata developed the idea that oscillation arise from the not equality between neutrino interaction and neutrino matter states [20]. The oscillations occur due to interference between different massive neutrinos. This idea was then further developed by Pontecorvo [21]. In this sense, the massive neutrinos, the matter eigenstates, are described as a superposition of the flavor neutrinos, the weak interaction eigenstates or vice versa.

Experimentally, the first hints of neutrino oscillations were observed in solar neutrino experiments and then confirmed by the observation of a reduction in the atmospheric neutrino flux in Super Kamiokande 22. The confirmation that one initial solar neutrino is converted into any other type of active neutrino came then in the observation of solar neutrinos by the SNO experiment [23]. This discovery lead to the Nobel prize in physics in 2015 [24, 25].

\subsection{Neutrino oscillations in vacuum}

In this section, we derive the formula for neutrino oscillations in vacuum. We will refer to the weak interaction eigenstates as $\nu_{\alpha}$, where in general $\alpha \in\{e, \mu, \tau\}$. These are the neutrinos who participate in charged current (CC) interactions, i.e. 
via $W^{ \pm}$-bosons, and neutral current $(\mathrm{NC})$ interactions, i.e. via $Z$-bosons. The massive neutrinos are referred to as $\nu_{i}$, where $i=1,2,3$. Hence, we use Greek indices for flavor neutrinos and Latin indices for the mass eigenstates. A flavor neutrino $\nu_{\alpha}$ can be described as a superposition of massive neutrinos,

$$
\left|\nu_{\alpha}\right\rangle=\sum_{k} U_{\alpha k}^{*}\left|\nu_{k}\right\rangle
$$

where each of the massive neutrinos is weighted with a factor $U_{\alpha k}^{*}$. These weights are given by the entries of a unitary matrix, called the lepton mixing matrix or PMNS (after Pontecorvo, Maki, Nakagawa and Sakata) matrix, $U$. The eigenstates satisfy

$$
\left\langle\nu_{\alpha} \mid \nu_{\beta}\right\rangle=\delta_{\alpha \beta}
$$

and because of the unitarity of the the lepton mixing matrix

$$
\left\langle\nu_{k} \mid \nu_{j}\right\rangle=\delta_{k j}
$$

In vacuum, the massive neutrinos are eigenstates of the (free) Hamiltonian, satisfying

$$
\mathcal{H}_{0}\left|\nu_{k}\right\rangle=E_{k}\left|\nu_{k}\right\rangle, \quad \text { with } \quad E_{k}=\sqrt{\vec{p}^{2}+m_{k}^{2}} .
$$

Therefore, the Schrödinger equation

$$
i \partial_{t}\left|\nu_{k}\right\rangle=\mathcal{H}_{0}\left|\nu_{k}\right\rangle
$$

has the solution

$$
\left|\nu_{k}(t)\right\rangle=e^{-i E_{k} t}\left|\nu_{k}\right\rangle .
$$

This behavior can be related directly to the weak eigenstates, because Eq. (1.1) holds at any time. Hence, we have

$$
\left|\nu_{\alpha}(t)\right\rangle=U_{\alpha k}^{*}\left|\nu_{k}(t)\right\rangle=\sum_{k} U_{\alpha k}^{*} e^{-i E_{k} t}\left|\nu_{k}\right\rangle,
$$

where $\left|\nu_{k}\right\rangle=\left|\nu_{k}(0)\right\rangle$. We can use the inverse of Eq. 1.1

$$
\left|\nu_{k}\right\rangle=\sum_{\alpha} U_{\alpha k}\left|\nu_{\alpha}\right\rangle
$$

and substitute into Eq. 1.7 to obtain 


$$
\left|\nu_{\alpha}(t)\right\rangle=\sum_{k, \beta} U_{\alpha k}^{*} U_{\beta k} e^{-i E_{k} t}\left|\nu_{\beta}\right\rangle .
$$

Therefore, the flavor neutrino at time $t$ is a superposition of the flavor eigenstates at time $t=0$. Using this last equation, we can easily calculate the transition amplitude for an initial neutrino of flavor $\alpha$ to oscillate into a flavor $\beta$

$$
A_{\alpha \beta}(t)=\left\langle\nu_{\beta} \mid \nu_{\alpha}(t)\right\rangle=\sum_{k} U_{\alpha k}^{*} U_{\beta k} e^{-i E_{k} t}
$$

and from here also the oscillation probability

$$
P_{\alpha \beta}(t)=\left|A_{\alpha \beta}(t)\right|^{2}=\sum_{k, j} U_{\alpha k}^{*} U_{\beta k} U_{\alpha j} U_{\beta j}^{*} e^{-i\left(E_{k}-E_{j}\right) t} .
$$

For relativistic neutrinos, the eigenvalues of the free Hamiltonian can be approximated as

$$
E_{k} \approx E+\frac{m_{k}^{2}}{2 E}
$$

where we used the equal momentum approximation, $E=\left|\vec{p}_{k}\right|$. Using also the fact that neutrinos travel very close to the speed of light and, therefore, $t=L$, where $L$ is the distance traveled, we obtain

$$
P_{\alpha \beta}(E, L)=\sum_{k, j} U_{\alpha k}^{*} U_{\beta k} U_{\alpha j} U_{\beta j}^{*} e^{i \frac{\Delta m_{k j}^{2}}{2 E} L},
$$

where $\Delta m_{k j}^{2}=m_{k}^{2}-m_{j}^{2}$ are the mass squared differences. We also write the probability now as a function of the energy $E$ and the baseline $L$. We can split the summation to obtain

$$
P_{\alpha \beta}(E, L)=\sum_{k}\left|U_{\alpha k}\right|^{2}\left|U_{\beta k}\right|^{2}+\sum_{k \neq j}\left[U_{\alpha k}^{*} U_{\beta k}\right]\left[U_{\alpha j} U_{\beta j}^{*}\right] e^{i \frac{\Delta m_{k j}^{2}}{2 E} L} .
$$

Note that, for $k \leftrightarrow j$, the term in the second sum gets replaced by its complex conjugate. Therefore, we have 


$$
\begin{aligned}
P_{\alpha \beta}(E, L)= & \sum_{k}\left|U_{\alpha k}\right|^{2}\left|U_{\beta k}\right|^{2}+2 \operatorname{Re}\left[\sum_{k>j} U_{\alpha k}^{*} U_{\beta k} U_{\alpha j} U_{\beta j}^{*} e^{i \frac{\Delta m_{k j}^{2}}{2 E} L}\right] \\
= & \sum_{k}\left|U_{\alpha k}\right|^{2}\left|U_{\beta k}\right|^{2}+2 \sum_{k>j} \operatorname{Re}\left[U_{\alpha k}^{*} U_{\beta k} U_{\alpha j} U_{\beta j}^{*}\right] \cos \left(\frac{\Delta m_{k j}^{2} L}{2 E}\right) \\
& -2 \sum_{k>j} \operatorname{Im}\left[U_{\alpha k}^{*} U_{\beta k} U_{\alpha j} U_{\beta j}^{*}\right] \sin \left(\frac{\Delta m_{k j}^{2} L}{2 E}\right) .
\end{aligned}
$$

We can finally use

$$
\sum_{k}\left|U_{\alpha k}\right|^{2}\left|U_{\beta k}\right|^{2}=\delta_{\alpha \beta}-2 \operatorname{Re} \sum_{k>j} U_{\alpha k}^{*} U_{\beta k} U_{\alpha j} U_{\beta j}^{*}
$$

and some trigonometrical manipulations to arrive at

$$
\begin{aligned}
P_{\alpha \beta}(E, L) & =\delta_{\alpha \beta}-4 \sum_{k>j} \operatorname{Re}\left[U_{\alpha k}^{*} U_{\beta k} U_{\alpha j} U_{\beta j}^{*}\right] \sin ^{2}\left(\frac{\Delta m_{k j}^{2} L}{4 E}\right) \\
& +2 \sum_{k>j} \operatorname{Im}\left[U_{\alpha k}^{*} U_{\beta k} U_{\alpha j} U_{\beta j}^{*}\right] \sin \left(\frac{\Delta m_{k j}^{2} L}{2 E}\right) .
\end{aligned}
$$

From this form, we see that in the $3 \nu$ case neutrino oscillations depend on the quantities $\Delta m_{21}^{2}$ and $\Delta m_{31}^{2}\left(\Delta m_{32}^{2}\right.$ is just a combination of these two) and also on the entries of the lepton mixing matrix $U$. Considering three massive neutrino states, this matrix can be written as the product of three different rotations,

$$
\begin{aligned}
U & =\left(\begin{array}{ccc}
1 & 0 & 0 \\
0 & c_{23} & s_{23} \\
0 & -s_{23} & c_{23}
\end{array}\right)\left(\begin{array}{ccc}
c_{13} & 0 & s_{13} e^{-i \delta} \\
0 & 1 & 0 \\
-s_{13} e^{i \delta} & 0 & c_{13}
\end{array}\right)\left(\begin{array}{ccc}
c_{12} & s_{12} & 0 \\
-s_{12} & c_{12} & 0 \\
0 & 0 & 1
\end{array}\right) \\
& =\left(\begin{array}{ccc}
c_{12} c_{13} & s_{12} c_{13} & s_{13} e^{-i \delta} \\
-s_{12} c_{23}-c_{12} s_{23} s_{13} e^{i \delta} & c_{12} c_{23}-s_{12} s_{23} s_{13} e^{i \delta} & s_{23} c_{13} \\
s_{12} s_{23}-c_{12} c_{23} s_{13} e^{i \delta} & -c_{12} s_{23}-s_{12} c_{23} s_{13} e^{i \delta} & c_{23} c_{13}
\end{array}\right)
\end{aligned}
$$

where $s_{i j}=\sin \theta_{i j}$ and $c_{i j}=\cos \theta_{i j}$. Therefore, the oscillations depend, apart from the mass splittings, on three angles $\theta_{23}, \theta_{13}, \theta_{12}$ and a phase $\delta$.

As a final remark, let us mention that it was shown that the imaginary parts in Eq. (1.17) are all equal up to a given sign, 


\begin{tabular}{|c|c|}
\hline Parameter & Value \\
\hline$\Delta m_{21}^{2}$ & $7.5 \times 10^{-5} \mathrm{eV}^{2}$ \\
$\Delta m_{e e}^{2}$ & $2.50 \times 10^{-3} \mathrm{eV}^{2}$ \\
$\sin ^{2} \theta_{12}$ & 0.32 \\
$\sin ^{2} \theta_{23}$ & 0.55 \\
$\sin ^{2} \theta_{13}$ & 0.022 \\
$\delta$ & $-0.40 \pi$ \\
\hline
\end{tabular}

Table 1.1: Neutrino oscillation parameters used in this chapter.

$$
\operatorname{Im}\left[U_{\alpha k}^{*} U_{\beta k} U_{\alpha j} U_{\beta j}^{*}\right]=s_{\alpha \beta k j} J,
$$

where $s_{\alpha \beta k j}= \pm 1$ and $J$ is the Jarlskog invariant

$$
J=\sin \delta s_{12} c_{12} s_{13} c_{13}^{2} s_{23} c_{23} .
$$

Therefore, the final form of the oscillation probability is given by

$$
\begin{aligned}
P(\alpha \rightarrow \beta, t) & =\delta_{\alpha \beta}-4 \sum_{k>j} \operatorname{Re}\left[U_{\alpha k}^{*} U_{\beta k} U_{\alpha j} U_{\beta j}^{*}\right] \sin ^{2}\left(\frac{\Delta m_{k j}^{2} L}{4 E}\right) \\
& +2 J \sum_{k>j} s_{\alpha \beta k j} \sin \left(\frac{\Delta m_{k j}^{2} L}{2 E}\right) .
\end{aligned}
$$

The factors $\operatorname{Re}\left[U_{\alpha k}^{*} U_{\beta k} U_{\alpha j} U_{\beta j}^{*}\right]$ and $s_{\alpha \beta k j}$ can be found nicely summarized in Tab. 13.1 of Ref. [26]. In Fig. 1.1 we show the oscillation probabilities for an original muon neutrino to oscillate into any of the flavors, where a baseline of $1300 \mathrm{~km}$ was assumed. The oscillation parameters used to create this plot are summarized in Tab. 1.11

\subsection{Neutrino oscillations in matter}

In the last section we discussed neutrino oscillations in vacuum. When traveling through matter, however, these oscillations are altered by effective potentials

\footnotetext{
${ }^{1}$ In chapter 2 we will go much more into detail on how to obtain these oscillation parameters. As of now, we only like to ensure that this choice of parameters is reasonable, as they are all within the $1 \sigma$ allowed regions.
} 


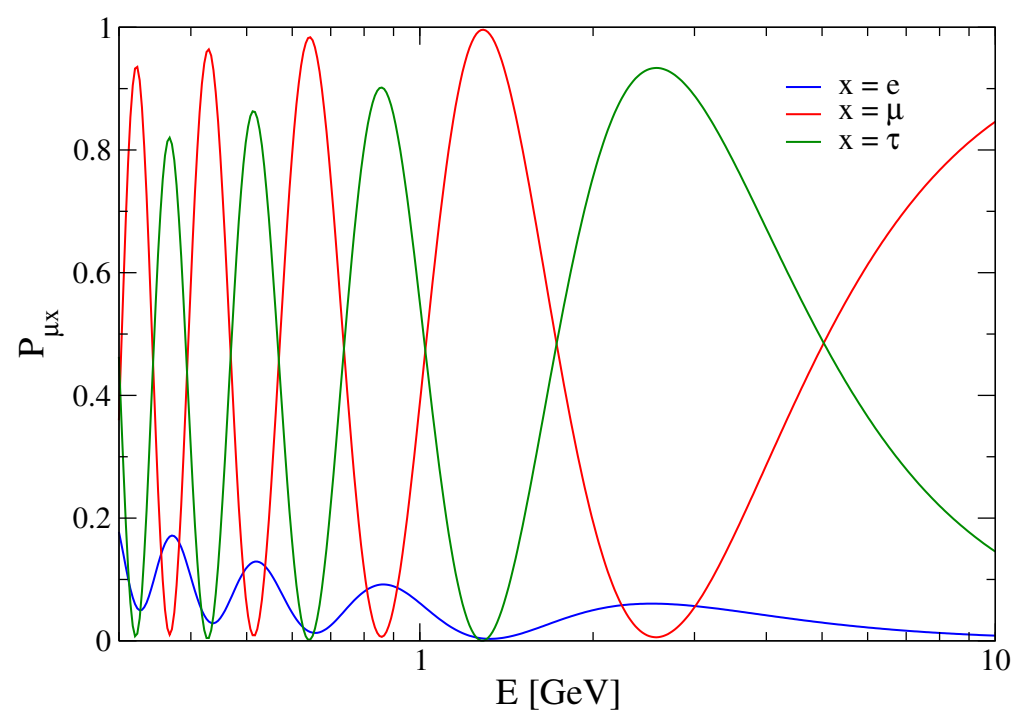

Figure 1.1: The probabilities for an original muon neutrino to be found in the different flavors after traveling $1300 \mathrm{~km}$ as functions of energy.

due to coherent forward scattering with the particles in the medium. The Feynman diagrams for charged current (CC) and neutral current (NC) processes are shown in Fig. 1.2 .

In this case, the Hamiltonian contains, apart from the free part $\mathcal{H}_{0}$, an interaction part $\mathcal{H}_{I}$ and is given by $\mathcal{H}=\mathcal{H}_{0}+\mathcal{H}_{I}$, where

$$
\mathcal{H}_{0}\left|\nu_{k}\right\rangle=E_{k}\left|\nu_{k}\right\rangle, \quad \text { and } \quad \mathcal{H}_{I}\left|\nu_{\alpha}\right\rangle=V_{\alpha}\left|\nu_{\alpha}\right\rangle .
$$

Note that $\mathcal{H}_{0}$ is diagonal in the mass basis while $\mathcal{H}_{I}$ is diagonal in the flavor basis. The matter potential $V_{\alpha}$ is given by 26]

$$
V_{\alpha}=V_{C C} \delta_{\alpha e}+V_{N C}=\sqrt{2} G_{F}\left(n_{e} \delta_{\alpha e}-\frac{n_{n}}{2}\right)
$$

with the electron and neutron number densities, $n_{e}$ and $n_{n}$ respectively. The Schrödinger equation for a flavor state is then

$$
i \partial_{t}\left|\nu_{\alpha}\right\rangle=\mathcal{H}\left|\nu_{\alpha}\right\rangle=\mathcal{H}_{0}\left|\nu_{\alpha}\right\rangle+V_{\alpha}\left|\nu_{\alpha}\right\rangle .
$$

To find a solution we shall work with the transition amplitudes $\psi_{\alpha \beta}(t)=\left\langle\nu_{\beta} \mid \nu_{\alpha}(t)\right\rangle$. Using the last equations we have 



Figure 1.2: The Feynman diagrams for CC (left) and NC (right) elastic scattering of neutrinos on electrons and nucleons.

$$
i \partial_{t} \psi_{\alpha \beta}(t)=\sum_{\gamma}\left(\sum_{k} U_{\beta k} E_{k} U_{\gamma k}^{*}+\delta_{\beta \gamma} V_{\beta}\right) \psi_{\alpha \gamma}(t) .
$$

Applying again the high-energy approximation for relativistic neutrinos we obtain

$$
\begin{aligned}
i \partial_{t} \psi_{\alpha \beta}(t) & =\left(E+\frac{m_{1}^{2}}{2 E}+V_{N C}\right) \psi_{\alpha \beta}(t) \\
& +\sum_{\gamma}\left(\sum_{k} U_{\beta k} \frac{\Delta m_{k 1}^{2}}{2 E} U_{\gamma k}^{*}+\delta_{\beta \gamma} \delta_{\beta e} V_{C C}\right) \psi_{\alpha \gamma}(t) .
\end{aligned}
$$

The first term, which contains the neutral current potential, would result only in a phase-factor in the transition amplitude and has no effect on the transition probability. Note that, therefore, the neutrino oscillation probability does not depend on the neutral current potential and the neutron number density in the medium. We are left with

$$
i \partial_{t} \psi_{\alpha \beta}(t)=\sum_{\gamma}\left(\sum_{k} U_{\beta k} \frac{\Delta m_{k 1}^{2}}{2 E} U_{\gamma k}^{*}+\delta_{\beta \gamma} \delta_{\beta e} V_{C C}\right) \psi_{\alpha \gamma}(t),
$$

which can be rewritten in matrix form as

$$
i \partial_{t} \Psi_{\alpha}=\mathcal{H}_{\mathrm{F}} \Psi_{\alpha}
$$


with

$$
\mathcal{H}_{\mathrm{F}}=\frac{1}{2 E}\left(U \mathbb{M}^{2} U^{\dagger}+\mathbb{A}\right)
$$

This matrix equation can be easily generalized to any number of neutrinos by simply extending the dimensions of the vectors and matrices in the equation. Also, the neutral current potential could be added if needed in the diagonal entries of the matrix $\mathbb{A}$ (for instance, when considering additional sterile neutrinos). In the case of three massive neutrino we have

$$
\Psi_{\alpha}=\left(\begin{array}{c}
\psi_{\alpha e} \\
\psi_{\alpha \mu} \\
\psi_{\alpha \tau}
\end{array}\right), \quad \mathbb{M}^{2}=\left(\begin{array}{ccc}
0 & 0 & 0 \\
0 & \Delta m_{21}^{2} & 0 \\
0 & 0 & \Delta m_{31}^{2}
\end{array}\right), \quad \mathbb{A}=\left(\begin{array}{ccc}
a & 0 & 0 \\
0 & 0 & 0 \\
0 & 0 & 0
\end{array}\right)
$$

where $a=2 \sqrt{2} E G_{F} n_{e}$ and $G_{F}$ is the Fermi constant. From this equations, it becomes clear that we will not be able to write down solutions in an easy manner as in the case of vacuum oscillation. In the most general case of a potential which depends on the position of the neutrino, one needs to discretize the neutrino path, calculate $a$ and diagonalize $\mathcal{H}_{F}$ at each step. These diagonalizations obviously can not be performed by hand and require specialized software.

However, if one assumes a constant matter density profile, exact and approximate solutions can be obtained. In the next section we will consider exactly this case, where a neutrino travels through matter with a constant potential along the neutrino path. Assuming a constant density profile is a very good and reasonable approximation in the context of long-baseline accelerator experiments, where neutrinos travel only through the outermost layer of the Earth density profile.

\subsection{Neutrino oscillations in constant matter}

In this section, we will focus on neutrino oscillations in a constant matter profile. In this scenario, an exact solution to the Schrödinger equation can, in principle, be found. Unfortunately, this solution is very complicated, due to terms containing $\cos \left[\frac{1}{3} \arccos (\ldots)\right]$, where a complicated function of all the oscillation parameters appears inside the arccos, not allowing for a deeper insight into the phenomenology of neutrino oscillations. This term can not be avoided, because it appears in the general exact solution of third order polynomial equations. Many analytic approximate solutions have been derived then, instead, to avoid this type of term. Here we will compare most of these expressions, derived in the last two decades. We will normalize the notation and compare them among each other in 
terms of precision and computation speed. The results of this section have been published in Ref. [1].

As seen in the last sections, we need to solve the Schrödinger equation in order to obtain the neutrino oscillation probabilities. While this was easily done in the case of vacuum oscillations (i.e. a free Hamiltonian), it becomes very difficult to do in the presence of matter due to alterations of the oscillation patterns due to the Mikheyev-Smirnov-Wolfenstein (MSW) effect [27-30]. However, if one assumes a approximately constant matter profile in the trajectory of the neutrino, analytic solutions can be found [31]. These expressions are very complicated, unfortunately, and do not permit for a deeper understanding of the phenomenology of three-neutrino oscillations.

Therefore, to obtain better insights one may try to find simpler analytic expansions around naturally appearing small parameters. Some commonly used small parameters are $a / \Delta m_{31}^{2}\left[32\right.$, $\sin \theta_{13}$ or $\sin ^{2} \theta_{13}$ [33, 34, and the ratio of mass splittings $\Delta m_{21}^{2} / \Delta m_{31}^{2}$ or $\Delta m_{21}^{2} / \Delta m_{e e}^{2} 32,35,40$, where $\Delta m_{e e}^{2} \equiv \cos ^{2} \theta_{12} \Delta m_{31}^{2}+$ $\sin ^{2} \theta_{12} \Delta m_{32}^{2}$ [41,42]. We analyze, with an eye for both precision and computational speed, different expansions and show how accurate they are and how they have aged as the measurement of the oscillation parameters has evolved over the past twenty years. It is clear that simplicity is an important trait for an approximate expression. While simplicity may be somewhat in the eye of the beholder, we use computational speed as a rough proxy for simplicity.

We focus on the Deep Underground Neutrino Experiment (DUNE) [43] experimental conditions $\mathrm{s}^{2}$ of $L=1300 \mathrm{~km}$ and Earth density of $\rho=3 \mathrm{~g} / \mathrm{cm}^{3}$. We take the matter density to be constant, which is a good approximation since effects due to the variability of the density of the Earth are beyond the sensitivity of DUNE [4]. The results will also generally apply to other long-baseline experiments such as NOVA [45], T2K/HK [46, 47]. We also discuss the second oscillation maximum which is relevant for T2HKK [48] and ESSnuSB [49].

\subsubsection{Expansions under consideration}

In this section, we present the expansions under consideration. We will later compare them to the exact result obtained from numerical integration. Before commenting on the prediction of each expression, we categorize them into three groups based on their forms. The first is the "Madrid-like" group named for the common city for which one expression was first written down. Other very similar expressions followed and they will be grouped together accordingly. The next

\footnotetext{
${ }^{2}$ We will go into more detail regarding the experimental configuration in further chapters. Here the only important things are the baseline length and matter density.
} 
group is the AKT, MP and DMP group. This is a series of works that performs two flavor rotations and then perturbation theory. The final group contains the remaining expressions. Expressions generally drop terms proportional to various smallness parameters including the ratio of $\Delta m^{2}$ 's, $s_{13}$, or the matter potential.

For historical reasons (i.e. neutrino factory [50]), many of these expansions have been performed in the channel $\nu_{e} \rightarrow \nu_{\mu}$. However, in the context of longbaseline accelerators the most important channel is $\nu_{\mu} \rightarrow \nu_{e}$. Therefore, we will present our results in this channel. They are related to each other through the T-relation $P\left(\nu_{\mu} \rightarrow \nu_{e} ; \delta\right)=P\left(\nu_{e} \rightarrow \nu_{\mu} ;-\delta\right)$, just switching the sign of the CPphas $\AA^{3}$. If one is interested in antineutrinos, namely $P\left(\bar{\nu}_{\mu} \rightarrow \bar{\nu}_{e} ; E\right)=P\left(\nu_{\mu} \rightarrow\right.$ $\left.\nu_{e} ;-E\right)$, one only has to switch the sign of the neutrino energy. We will focus on neutrinos, but our results generally apply to antineutrinos as well. Note that matter effects are not very important in the disappearance channels $\nu_{\mu} \rightarrow \nu_{\mu}$ and, therefore, focusing only on the appearance channel will also not affect the main message.

While the choice of notation does not affect the precision of these formulas, it does affect their general clarity and overall usefulness. To this end, we have chosen to use uniform notation throughout this chapter as much as possible with the various terms defined in Tab. 1.2. In doing so we have made several simplifying manipulations, in each case maintaining the exact same mathematical expression. The relationship between the notation used here and the original notation used is mentioned below whenever applicable. While these definitions represent fairly commonly used definitions in the literature, some differ by factors of two or other slight changes, so care is required when making comparisons.

\subsubsection{The Madrid-like expressions}

Here we list a few nearly identical expressions and discuss their similarities and differences.

The Madrid expression (2000) This expression was derived in Ref. 33 (by Cervera, Donini, Gavela, Gomez Cádenas, Hernández, Mena, and Rigolin,

\footnotetext{
${ }^{3}$ Note that sending $\delta \rightarrow-\delta$ is equivalent to sending $L \rightarrow-L$ under the assumption of CPT invariance.
} 


\begin{tabular}{|l|l|}
\hline$E$ & Neutrino energy \\
$L$ & Baseline \\
\hline$a$ & $2 E \sqrt{2} G_{F} n_{e}$ \\
$G_{F}$ & Fermi's constant \\
$n_{e}$ & Electron \# density \\
\hline$s_{i j}$ & $\sin \theta_{i j}$ \\
$c_{i j}$ & $\cos \theta_{i j}$ \\
$\Delta m_{i j}^{2}$ & $m_{i}^{2}-m_{j}^{2}$ \\
$\Delta m_{e e}^{2}$ & $c_{12}^{2} \Delta m_{31}^{2}+s_{12}^{2} \Delta m_{32}^{2}$ \\
\hline$\Delta_{i j}$ & $\Delta m_{i j}^{2} L /(4 E)$ \\
$\Delta_{x}$ & $x L /(4 E)$ \\
\hline$\epsilon$ & $\Delta m_{21}^{2} / \Delta m_{e e}^{2}$ \\
$\varepsilon$ & $\Delta m_{21}^{2} / \Delta m_{31}^{2}$ \\
\hline$J_{r}$ & $s_{23} c_{23} s_{13} c_{13}^{2} s_{12} c_{12}$ \\
\hline$\widehat{x}$ & $x$ in matter \\
$\widetilde{x}$ & Approx. $x$ in matter \\
\hline
\end{tabular}

Table 1.2: The various terms used throughout this chapter.

Madrid hereafter) and can be written $\left.\right|^{4}$

$$
\begin{aligned}
P_{\mu e}= & 4 s_{23}^{2} s_{13}^{2} c_{13}^{2}\left(\frac{\Delta m_{31}^{2}}{b}\right)^{2} \sin ^{2} \Delta_{b}+4 c_{23}^{2} s_{12}^{2} c_{12}^{2}\left(\frac{\Delta m_{21}^{2}}{a}\right)^{2} \sin ^{2} \Delta_{a} \\
& +8 J_{r} \frac{\Delta m_{21}^{2}}{a} \frac{\Delta m_{31}^{2}}{b} \sin \Delta_{a} \sin \Delta_{b} \cos \left(\delta+\Delta_{31}\right),
\end{aligned}
$$

where $b \equiv a-\Delta m_{31}^{2}$.

The form of this expression suggests the square of two summed amplitudes. It is not exactly such a sum due to an extra factor of $c_{13}$ in the interference term which provides the correct CP-violating term in vacuum. Writing Eq. (1.32) as the sum of two amplitudes has been examined in various forms in Refs. [37,51,53].

There are two ways to correct this. The first is to drop one of the $c_{13}$ 's in the Jarlskog invariant $\left(J_{r}\right)$. Alternatively, if we add a factor of $c_{13}$ to one of the amplitudes, we recover the Jarlskog in vacuum correctly while still writing the

\footnotetext{
${ }^{4}$ In Ref. 33$] \tilde{J} \equiv 8 J_{r}$ is used instead, the definition of $\Delta_{i j}$ differs by a factor of $L / 2$, and terms $A \equiv a /(2 E)$ and term $B \equiv|b| /(2 E)$ are used. While Ref. 33 defines their $b$-like parameter with absolute value signs, we note that they are not necessary since the probability is even in $b$.
} 
expression as the sum of two amplitudes. The natural place to put it is on the $a$ (21) term [51 54 as this reproduces the vacuum expression exactly. We note that this provides a negligible change to the precision of the equation as we are correcting an already subleading term (the solar term) by a small amount. On the other hand we can add the $c_{13}$ term to the $b$ (31) term. Doing so improves the precision of the Madrid expression for neutrinos by about an order of magnitude at the first oscillation maximum, although this effect is due to a lucky cancellation for the parameters used. The improvement is more modest elsewhere, and for antineutrinos the precision is a bit worse than the original Madrid expression. As such, we do not include such an expression in our subsequent analyses.

In addition, considering the previously identified importance of $\Delta m_{e e}^{2}[42$ and the fact that there is no reason to use $\Delta m_{31}^{2}$ or $\Delta m_{32}^{2}$ unless both are treated separately, we have also examined how Eq. 1.32 performs with $\Delta m_{31}^{2} \rightarrow \Delta m_{e e}^{2}$. We find that this change results in somewhat better performance in some cases (modest improvement at $E \gtrsim$ few $\mathrm{GeV}$ and considerable improvement at and below the second maximum). However, in the region of interest for DUNE around a few $\mathrm{GeV}$ the performance is essentially the same as the Madrid expression.

The AJLOS(31) expression (2004) In Ref. [36] (by Akhmedov, Johansson, Lindner, Ohlsson, and Schwetz, AJLOS hereafter), several expressions are introduced each with different expansion parameters. We label them by the equation numbers in the original paper 5 .

The first expression (\#31) drops higher order terms proportional to $\varepsilon$ and $s_{13}$.

$$
\begin{aligned}
P_{\mu e}= & 4 s_{13}^{2} s_{23}^{2}\left(\frac{\Delta m_{31}^{2}}{b}\right)^{2} \sin ^{2} \Delta_{b}+4 s_{12}^{2} c_{12}^{2} c_{23}^{2}\left(\frac{\Delta m_{21}^{2}}{a}\right)^{2} \sin ^{2} \Delta_{a} \\
& +8 \frac{J_{r}}{c_{13}^{2}} \frac{\Delta m_{21}^{2}}{a} \frac{\Delta m_{31}^{2}}{b} \sin \Delta_{a} \sin \Delta_{b} \cos \left(\Delta_{31}+\delta\right) .
\end{aligned}
$$

We note that, up to a factor of $c_{13}^{2}$ in each the second and third term, this expression is otherwise identical to the Madrid expression in Eq. 1.32.

The FL expression (2006) Also the authors of Ref. 37] (Friedland and Lunardini, FL hereafter) write the probability as the sum of two amplitudes. Using

\footnotetext{
${ }^{5}$ In Ref. 36] $\alpha=\varepsilon, A=a / \Delta m_{31}^{2}$, and $\Delta=\Delta_{31}$ are used. We refer to the equation numbers in the version on the arXiv, not in the journal.
} 
our notation 6 , they obtain

$$
P_{\mu e}=\left|\Delta_{32} e^{i \delta} s_{13} c_{13} s_{23} \frac{e^{2 i\left(\Delta_{32}-\Delta_{a}\right)}-1}{\Delta_{32}-\Delta_{a}}-\Delta_{21} s_{12} c_{12} c_{23} \frac{e^{-2 i \Delta_{a}}-1}{\Delta_{a}}\right|^{2} .
$$

Note that, this expression was derived actually in the context of Non-Standard neutrino Interactions (NSI) and that it reduces to Eq. (1.34) once all the NSI parameters are set to zero. This expression is identical to Eq. 11.32 up to using $\Delta m_{32}^{2}$ instead of $\Delta m_{31}^{2}$ and factors of $c_{13}$.

\subsubsection{The AKT, MP and DMP expressions}

In Refs. 38, 39,55], a different technique was used. Two-flavor rotations were performed to simply diagonalize the Hamiltonian by focusing on the largest offdiagonal terms first. This means that all channels $\left(\nu_{\alpha} \rightarrow \nu_{\beta}\right.$ for $\left.\alpha, \beta \in\{e, \mu, \tau\}\right)$ are handled simultaneously. In AKT, the focus is on the vacuum mass eigenstate basis whereas MP and DMP focus on the flavor basis. This choice affects the order of the two two-flavor rotations and, hence, the precision. Roughly speaking, AKT performs a 12 rotation followed by a 13 rotation whereas MP and DMP first perform a 13 rotation followed by a 12 rotation as sketched below.

The AKT expression (2014) The authors (Agarwalla, Kao, and Takeuchi) of Ref. [55] (AKT hereafter) perform the rotations going from the largest contribution in the Hamiltonian to the smallest one in the mass basis. They begin with the 12 rotation, followed by the 23 rotation and then, after commuting with the 12 rotation, they absorb this 23 rotation into a 13 rotation.

Using this approach, the effective mixing angles can be written as

$$
\begin{aligned}
\tan 2 \widetilde{\theta}_{12} & =\frac{\Delta m_{21}^{2} \sin 2 \theta_{12}}{\Delta m_{21}^{2} \cos 2 \theta_{12}-a c_{13}^{2}}, \\
\tan 2 \widetilde{\theta}_{13} & =\frac{\Delta m_{e e}^{2} \sin 2 \theta_{13}}{\Delta m_{e e}^{2} \cos 2 \theta_{13}-a} .
\end{aligned}
$$

\footnotetext{
${ }^{6}$ In Ref. 37. $\Delta_{1}=2\left(\Delta_{32}-\Delta_{a}\right), \Delta_{2}=-2 \Delta_{a}, G_{1}=\Delta_{32} \sin 2 \theta_{13} e^{i \delta}$, and $G_{2}=-\Delta_{21} \sin 2 \theta_{12}$ were used.
} 
Note that $\delta$ and $\theta_{23}$ are treated as constant in matter. The eigenvalues of the Hamiltonian are now given by

$$
\begin{aligned}
& \lambda_{1 / p}=\frac{\left(\Delta m_{21}^{2}+a c_{13}^{2}\right)(-/+) \sqrt{\left(\Delta m_{21}^{2}-a c_{13}^{2}\right)^{2}+4 a c_{13}^{2} s_{12}^{2} \Delta m_{21}^{2}}}{2}, \\
& \lambda_{2 / 3}=\frac{\left(\lambda_{p}+\Delta m_{31}^{2}+a s_{13}^{2}\right)(-/+) \sqrt{\left(\lambda_{p}-\Delta m_{31}^{2}-a s_{13}^{2}\right)^{2}+4 a^{2} s_{\widetilde{12}}^{2} c_{13}^{2} s_{13}^{2}}}{2} .
\end{aligned}
$$

From these expressions we obtain the mass splittings in matter simply via the relation $\widetilde{\Delta m^{2}} i j=\lambda_{i}-\lambda_{j}$. The oscillation probability can now be obtained by replacing the vacuum parameters with the matter parameters in the vacuum oscillation probability, see Eqs. 1.42 and 1.43 below. While $\Delta m_{e e}^{2}$ was not explicitly used in Ref. [55, it appears in several places nonetheless and we made the substitution here for simplicity.

The MP expression (2015) After one rotation in the 13 sector, we have an expression that is an expansion in $\epsilon c_{12} s_{12}$. This is the expression in Ref. [38] (by Minakata and Parke, MP hereafter). The expression is

$$
\begin{aligned}
P_{\mu e}= & \left\{s_{23}^{2} \sin ^{2} 2 \theta_{13}+4 \epsilon J_{r} \cos \delta\left[\frac{\left(\lambda_{+}-\lambda_{-}\right)-\left(\Delta m_{e e}^{2}-a\right)}{\left(\lambda_{+}-\lambda_{0}\right)}\right]\right\} \\
& \times\left(\frac{\Delta m_{e e}^{2}}{\lambda_{+}-\lambda_{-}}\right)^{2} \sin ^{2} \frac{\left(\lambda_{+}-\lambda_{-}\right) L}{4 E} \\
& +8 \epsilon J_{r} \frac{\left(\Delta m_{e e}^{2}\right)^{3}}{\left(\lambda_{+}-\lambda_{-}\right)\left(\lambda_{+}-\lambda_{0}\right)\left(\lambda_{-}-\lambda_{0}\right)} \\
& \times \sin \frac{\left(\lambda_{+}-\lambda_{-}\right) L}{4 E} \sin \frac{\left(\lambda_{-}-\lambda_{0}\right) L}{4 E} \cos \left(\delta+\frac{\left(\lambda_{+}-\lambda_{0}\right) L}{4 E}\right) .
\end{aligned}
$$

where7

$$
\begin{aligned}
& \lambda_{0}=\epsilon \cos 2 \theta_{12} \Delta m_{e e}^{2}, \quad \lambda_{ \pm}=\frac{1}{2}\left[\Delta m_{e e}^{2}+a \pm \Delta \widetilde{m^{2}} e e\right] \\
& \text { with } \quad \widetilde{\Delta m^{2}} e \Delta \equiv \Delta m_{e e}^{2} \sqrt{\left(\cos 2 \theta_{13}-a / \Delta m_{e e}^{2}\right)^{2}+\sin ^{2} 2 \theta_{13}}=\lambda_{+}-\lambda_{-} .
\end{aligned}
$$

This expression is very accurate except near the solar resonance when $\Delta_{21}>1$.

${ }^{7}$ In Ref. 38 $\Delta m_{\mathrm{ren}}^{2}=\Delta m_{e e}^{2}$ is used and the definition of the eigenvalues is shifted by $\epsilon \Delta m_{e e}^{2} s_{12}^{2}$. 
The DMP expression (2016) To address the solar resonance, an additional rotation was performed in Ref. [39] (by Denton, Minakata, and Parke, DMP hereafter). The order of the rotations (12 then 13 after an initial constant 23 rotation) is chosen to diagonalize the largest remaining off-diagonal term at each step. This procedure also removes both level crossings with the minimal number of new angles. After these two rotations, perturbation theory is now possible everywhere, although the zeroth order expression $\left(\mathrm{DMP}^{0}\right)$ is sufficiently precise for future long-baseline experiments.

Here, as in AKT in section 1.3.1.2 above, the authors do not derive formulas for the oscillation probabilities directly, but rather for the oscillation parameters in matter and then write the probability

$$
\widetilde{P}_{\alpha \beta}\left(\Delta m_{i j}^{2}, \theta_{i j}, \delta\right)=P_{\alpha \beta}\left(\Delta \widetilde{m^{2}}{ }_{i j}, \widetilde{\theta}_{i j}, \widetilde{\delta}\right),
$$

where $\widetilde{x}$ refers to the approximate expression for the quantity $x$ evaluated in matter. That is, to an excellent approximation the oscillation probability in matter has the same form as the expression in vacuum with only $\Delta m_{21}^{2}, \Delta m_{31}^{2}$, $\theta_{12}$, and $\theta_{13}$ replaced by their approximate matter equivalents $\left(\widetilde{\theta}_{23}\right.$ and $\widetilde{\delta}$ are roughly constant in matter). The authors obtain to zeroth order the following expressions 8

$$
\begin{aligned}
\sin ^{2} \widetilde{\theta}_{13} & =\frac{1}{2}\left(1-\frac{\Delta m_{e e}^{2} \cos 2 \theta_{13}-a}{\Delta{\widetilde{m^{2}}}_{e e}}\right), \\
\Delta \widetilde{m^{2}}{ }_{e e} & =\Delta m_{e e}^{2} \sqrt{\left(\cos 2 \theta_{13}-a / \Delta m_{e e}^{2}\right)^{2}+\sin ^{2} 2 \theta_{13}}, \\
\sin ^{2} \widetilde{\theta}_{12} & =\frac{1}{2}\left(1-\frac{\Delta m_{21}^{2} \cos 2 \theta_{12}-a_{12}}{\Delta \widetilde{m}^{2}}\right), \\
\text { where } \quad a_{12} & =\frac{1}{2}\left(a+\Delta m_{e e}^{2}-\Delta{\widetilde{m^{2}}}_{e e}\right), \\
\Delta \widetilde{m^{2}}{ }_{21} & =\Delta m_{21}^{2} \sqrt{\left(\cos 2 \theta_{12}-a_{12} / \Delta m_{21}^{2}\right)^{2}+\cos ^{2}\left(\theta_{13}-\widetilde{\theta}_{13}\right) \sin ^{2} 2 \theta_{12}}, \\
\Delta \widetilde{m^{2}} & =\Delta m_{31}^{2}+\frac{a}{4}+\frac{1}{2}\left(\widetilde{m^{2}} 21-\Delta m_{21}^{2}\right)+\frac{3}{4}\left(\Delta \widetilde{m^{2}}{ }_{e e}-\Delta m_{e e}^{2}\right) .
\end{aligned}
$$

${ }^{8}$ In Ref. 39 $\phi=\widetilde{\theta}_{13}, \psi=\widetilde{\theta}_{12}, \lambda_{i}=\widetilde{m^{2}}{ }_{i}$ were used. The current notation is consistent with Ref. 56. The following expression from 39 is also useful: $\cos ^{2}\left(\theta_{13}-\widetilde{\theta}_{13}\right)=c_{\widetilde{13}}^{2} c_{13}^{2}+s_{\widetilde{13}}^{2} s_{13}^{2}+$ $\sin 2 \widetilde{\theta}_{13} c_{13} s_{13}=\left(\widetilde{m^{2}} e e+\Delta m_{e e}^{2}-a \cos 2 \theta_{13}\right) /\left(2 \Delta \widetilde{m^{2}} e e\right)$. 
The expansion parameter is

$$
\epsilon^{\prime} \equiv \epsilon \sin \left(\widetilde{\theta}_{13}-\theta_{13}\right) s_{12} c_{12}<0.015
$$

and is zero in vacuum confirming that this expression returns the exact expression in that case.

We recall the vacuum expression here, which we write in the following form,

$P_{\mu e}=4 \mathbb{C}_{21} \sin ^{2} \Delta_{21}+4 \mathbb{C}_{31} \sin ^{2} \Delta_{31}+4 \mathbb{C}_{32} \sin ^{2} \Delta_{32}+8 \mathbb{D} \sin \Delta_{21} \sin \Delta_{31} \sin \Delta_{32}$,

where the coefficients are,

$$
\begin{aligned}
\mathbb{C}_{21} & =c_{13}^{2} s_{12}^{2} c_{12}^{2}\left(c_{23}^{2}-s_{13}^{2} s_{23}^{2}\right)+\cos 2 \theta_{12} J_{r} \cos \delta \\
\mathbb{C}_{31} & =s_{13}^{2} c_{13}^{2} c_{12}^{2} s_{23}^{2}+J_{r} \cos \delta \\
\mathbb{C}_{32} & =s_{13}^{2} c_{13}^{2} s_{12}^{2} s_{23}^{2}-J_{r} \cos \delta \\
\mathbb{D} & =-J_{r} \sin \delta .
\end{aligned}
$$

$\mathrm{DMP}^{0}$ is then given by Eqs. 1.42 , 1.43), where the vacuum parameters are replaced with the approximate matter ones given in Eq. 1.40).

Also note that $\Delta \widetilde{m^{2}}$ ee (which was further explored in $\left.\mid 56\right]$ ) is the same as $\lambda_{+}-\lambda_{-}$in Eq. (1.38) above from the MP formula in 1.3.1.2 Successive orders of precision can also be calculated by following perturbation theory in a straightforward fashion as is done through second order in 39 with compact expressions provided through first order or by correcting the mixing angles directly [57. Here we focus on zeroth and first orders only $\left(\mathrm{DMP}^{0}\right.$ and $\mathrm{DMP}^{1}$ hereafter respectively) as they are already extremely precise. Successive orders add $\sim 2.5$ additional orders of magnitude of precision if desired and expressions through second order exist in [39]. The first order corrections to the coefficients from Eq. (1.43) are 
given by the following expressions,

$$
\begin{aligned}
& \mathbb{C}_{21}^{(1)}=\epsilon^{\prime} \Delta m_{e e}^{2}\left(\frac{F_{1}}{\Delta \widetilde{m}^{2} 31}+\frac{F_{2}}{\Delta \widetilde{m^{2} 32}}\right), \\
& \mathbb{C}_{31}^{(1)}=\epsilon^{\prime} \Delta m_{e e}^{2}\left(\frac{F_{1}+G_{1}}{\Delta \widetilde{m}^{2} 31}-\frac{F_{2}}{\Delta \widetilde{m^{2}} 32}\right), \\
& \mathbb{C}_{32}^{(1)}=\epsilon^{\prime} \Delta m_{e e}^{2}\left(-\frac{F_{1}}{\Delta \widetilde{m}^{2} 31}+\frac{F_{2}+G_{2}}{\Delta \widetilde{m}^{2} 32}\right) \text {, } \\
& \mathbb{D}^{(1)}=\epsilon^{\prime} \Delta m_{e e}^{2}\left(\frac{K_{1}}{\widetilde{\Delta m^{2} 31}}-\frac{K_{2}}{\Delta \widetilde{m^{2} 32}}\right) \text {, }
\end{aligned}
$$

where $\epsilon^{\prime} \equiv \epsilon \sin \left(\widetilde{\theta}_{13}-\theta_{13}\right) s_{12} c_{12}$ as shown in Eq. (1.41) above, and

$$
\begin{aligned}
& F_{1}=c_{\widetilde{13}} s_{\widetilde{12}}^{2}\left[s_{\widetilde{13}} s_{\widetilde{12}} c_{\widetilde{12}}\left(c_{23}^{2}+\cos 2 \widetilde{\theta}_{13} s_{23}^{2}\right)-s_{23} c_{23}\left(s_{\widetilde{13}}^{2} s_{\widetilde{12}}^{2}+\cos 2 \widetilde{\theta}_{13} c_{\widetilde{12}}^{2}\right) \cos \delta\right] \\
& G_{1}=-2 s_{\widetilde{13}} c_{\widetilde{13}} s_{\widetilde{12}}\left(s_{23}^{2} \cos 2 \widetilde{\theta}_{13} c_{\widetilde{12}}-s_{23} c_{23} s_{\widetilde{13}} s_{\widetilde{12}} \cos \delta\right) \\
& K_{1}=-s_{23} c_{23} c_{\widetilde{13}} s_{\widetilde{12}}^{2}\left(c_{\widetilde{13}}^{2} c_{\widetilde{12}}^{2}-s_{\widetilde{13}}^{2}\right) \sin \delta
\end{aligned}
$$

and the $F_{2}, G_{2}, K_{2}$ expressions are related to the above by making the transformation $c_{\widetilde{12}}^{2} \leftrightarrow s_{\widetilde{12}}^{2}, c_{\widetilde{12}} s_{\widetilde{12}} \rightarrow-c_{\widetilde{12}} s_{\widetilde{12}}$, and $m_{1} \leftrightarrow m_{2}$. This correction is $\mathrm{DMP}^{1}$. Note that the expressions of Eq. 1.43) are also invariant under this transformation 39 .

\subsubsection{Other expressions}

Here we list other expressions in the literature that do not fall into the above two categories.

The AKS expression (1999) The oldest expression we consider is the one derived in Ref. 32 (by Arafune, Koike, and Sato, AKS hereafter). Here the 
authors obtain

$$
\begin{aligned}
P_{\mu e}= & 4 \sin ^{2} \Delta_{31} c_{13}^{2} s_{13}^{2} s_{23}^{2}\left(1+\frac{2 a}{\Delta m_{31}^{2}} \cos 2 \theta_{13}\right) \\
& +4 \Delta_{31} \sin \left(2 \Delta_{31}\right) c_{13}^{2} s_{13} s_{23} \\
& \times\left\{-\frac{a}{\Delta m_{31}^{2}} s_{13} s_{23} \cos 2 \theta_{13}+\varepsilon s_{12}\left(-s_{13} s_{23} s_{12}+\cos \delta c_{23} c_{12}\right)\right\} \\
& -8 J_{r} \Delta_{21} \sin ^{2} \Delta_{31} \sin \delta .
\end{aligned}
$$

The MF expression (2001) In Ref. [35] (by Freund, MF hereafter) the author separates the oscillation probability 9 in sub-terms, $P_{\mu e}=P_{0}+P_{\sin \delta}+P_{\cos \delta}+$ $P_{1}+P_{2}+P_{3}$. These terms are given by 10

$$
\begin{aligned}
P_{0}= & \frac{4 s_{23}^{2} s_{13}^{2} c_{13}^{2}}{C_{13}^{2}} \sin ^{2}\left(\Delta_{31} C_{13}\right), \\
P_{\sin \delta}= & -4 \sin \delta \frac{\Delta m_{21}^{2}}{a} \frac{s_{12} c_{12} s_{13} s_{23} c_{23}}{C_{13}} \sin \left(C_{13} \Delta_{31}\right) \\
& \times\left[\cos \left(C_{13} \Delta_{31}\right)-\cos \left(\Delta_{31}+\Delta_{a}\right)\right] \\
P_{\cos \delta}= & -4 \cos \delta \frac{\Delta m_{21}^{2}}{a} \frac{s_{12} c_{12} s_{13} s_{23} c_{23}}{C_{13}} \sin \left(C_{13} \Delta_{31}\right), \\
& \times\left[\sin \left(C_{13} \Delta_{31}\right)-\sin \left(\Delta_{31}+\Delta_{a}\right)\right] \\
P_{1}= & -4 \varepsilon \frac{1-\frac{a}{\Delta m_{31}^{2}} \cos 2 \theta_{13}}{C_{13}^{3}} s_{12}^{2} s_{13}^{2} c_{13}^{2} s_{23}^{2} \Delta_{31} \sin \left(2 \Delta_{31} C_{13}\right) \\
& -4 \varepsilon \frac{2 \frac{a}{\Delta m_{31}^{2}}\left(\frac{a}{\Delta m_{31}^{2}}-\cos 2 \theta_{13}\right)}{C_{13}^{4}} s_{12}^{2} s_{13}^{2} c_{13}^{2} s_{23}^{2} \sin ^{2}\left(\Delta_{31} C_{13}\right), \\
P_{2}= & 4 \frac{\Delta m_{21}^{2}}{a} \frac{C_{13}+\frac{a}{\Delta m_{31}^{2}} \cos 2 \theta_{13}-1}{C_{13}^{2}} s_{12} c_{12} s_{13} s_{23} c_{23} \sin ^{2}\left(\Delta_{31} C_{13}\right), \\
P_{3}= & 8\left(\frac{\Delta m_{21}^{2}}{a}\right)^{2} \frac{C_{13} c_{23}^{2} s_{12}^{2} c_{12}^{2}}{\cos ^{2} \theta_{13}\left(C_{13}+\cos _{2} 2 \theta_{13}-a / \Delta m_{31}^{2}\right)} \sin ^{2}\left[\frac{1}{2}\left(1-C_{13}\right) \Delta_{31}+\frac{1}{2} \Delta_{a}\right] .
\end{aligned}
$$

The expression in Eq. 1.47) is similar to the AJLOS(48) expression in Eq. 1.48 below.

\footnotetext{
${ }^{9}$ We use Eq. (36) of Ref. 35] since Eq. (38) behaves poorly for large values of $\theta_{13}$.

${ }^{10}$ In Ref. $35 . \hat{C}=C_{13}$ is used and there is a typo wherein $\cos \theta_{13}^{2}$ is written instead of $\cos ^{2} \theta_{13}$.
} 
The AJLOS(48) expression (2004) The second AJLOS (Ref. [36]) expression (\#48) focuses only on $\varepsilon$ as a smallness parameter. The first two orders, $P_{\mu e}=P_{\mu e}^{(0)}+\varepsilon P_{\mu e}^{(1)}$, are

$$
\begin{aligned}
P_{\mu e}^{(0)}= & 4 s_{23}^{2} s_{13}^{2} c_{13}^{2} \frac{\sin ^{2}\left(C_{13} \Delta_{31}\right)}{C_{13}^{2}} \\
P_{\mu e}^{(1)}= & -8 s_{12}^{2} s_{23}^{2} s_{13}^{2} c_{13}^{2} \frac{\sin \left(C_{13} \Delta_{31}\right)}{C_{13}^{2}}\left[\Delta_{31} \frac{\cos \left(C_{13} \Delta_{31}\right)}{C_{13}}\left(1-\frac{a}{\Delta m_{31}^{2}} \cos 2 \theta_{13}\right)\right. \\
& \left.-\frac{a}{\Delta m_{31}^{2}} \frac{\sin \left(C_{13} \Delta_{31}\right)}{C_{13}} \frac{\cos 2 \theta_{13}-a / \Delta m_{31}^{2}}{C_{13}}\right]+4 s_{13} s_{12} c_{12} s_{23} c_{23} \\
& \times \frac{\Delta m_{31}^{2} \sin \left(C_{13} \Delta_{31}\right)}{a C_{13}^{2}}\left\{\sin \delta\left[\cos \left(\Delta_{31}+\Delta_{a}\right)-\cos \left(C_{13} \Delta_{31}\right)\right] C_{13}\right. \\
& \left.+\cos \delta\left[C_{13} \sin \left(\Delta_{31}+\Delta_{a}\right)-\left(1-\frac{a}{\Delta m_{31}^{2}} \cos 2 \theta_{13}\right) \sin \left(C_{13} \Delta_{31}\right)\right]\right\},
\end{aligned}
$$

where $C_{13} \equiv \sqrt{\sin ^{2} 2 \theta_{13}+\left(a / \Delta m_{31}^{2}-\cos 2 \theta_{13}\right)^{2}}$ (this factor is also used in MF in the previous subsection, 1.3.1.3. We note that $C_{13}$ is equivalent to $\Delta \widetilde{m^{2}}{ }_{e e} / \Delta m_{e e}^{2}$ from DMP [39,56] after the change $\Delta m_{31}^{2} \rightarrow \Delta m_{e e}^{2}$.

There is also a third expression in the AJLOS paper, Eq. (66), but this is designed for the solar sector and does quite poorly for long-baseline oscillations which are dominated by the atmospheric term. For this reason we do not consider it here.

The AM expression (2011) We study the expression obtained by the authors of Ref. 34 (by Asano and Minakata, AM hereafter). Here the authors use as expansion parameters $s_{13} \simeq \sqrt{\Delta m_{21}^{2} / \Delta m_{31}^{2}}$. The authors divide their expressions in powers of $s_{13}, P_{\mu e}^{(0)}+P_{\mu e}^{(1)}+P_{\mu e}^{(3 / 2)}+P_{\mu e}^{(2)}+P_{\mu e}^{(5 / 2)}+\ldots$ Each superscript refers 
to the order of $s_{13}^{2}$ and $\Delta m_{21}^{2} / \Delta m_{31}^{2}$. For our channel of interest they obtain 11

$$
\begin{aligned}
P_{\mu e}^{(0)}= & 0 \\
P_{\mu e}^{(1)}= & 4 s_{23}^{2} s_{13}^{2} \frac{\sin ^{2}\left[\left(1-r_{A}\right) \Delta_{31}\right]}{\left(1-r_{A}\right)^{2}} \\
P_{\mu e}^{(3 / 2)}= & 8 J_{r} \frac{\varepsilon}{r_{A}\left(1-r_{A}\right)} \cos \left(\delta+\Delta_{31}\right) \sin \left(r_{A} \Delta_{31}\right) \sin \left[\left(1-r_{A}\right) \Delta_{31}\right] \\
P_{\mu e}^{(2)}= & 4 c_{23}^{2} c_{12}^{2} s_{12}^{2}\left(\frac{\varepsilon}{r_{A}}\right)^{2} \sin ^{2}\left(r_{A} \Delta_{31}\right) \\
& -4 s_{23}^{2}\left[s_{13}^{4} \frac{\left(1+r_{A}\right)^{2}}{\left(1-r_{A}\right)^{4}}-2 s_{12}^{2} s_{13}^{2} \frac{\varepsilon r_{A}}{\left(1-r_{A}\right)^{3}}\right] \sin ^{2}\left[\left(1-r_{A}\right) \Delta_{31}\right] \\
& +4 s_{23}^{2}\left[2 s_{13}^{4} \frac{r_{A}}{\left(1-r_{A}\right)^{3}}-s_{12}^{2} s_{13}^{2} \frac{\varepsilon}{\left(1-r_{A}\right)^{2}}\right] \Delta_{31} \sin \left[2\left(1-r_{A}\right) \Delta_{31}\right],
\end{aligned}
$$

where $r_{A} \equiv a / \Delta m_{31}^{2}$. The $5 / 2$ order term is given by

$$
\begin{aligned}
P_{\mu e}^{(5 / 2)}= & 8 J_{r} s_{13}^{2} \frac{\varepsilon r_{A}}{\left(1-r_{A}\right)^{3}} \cos \delta \sin ^{2}\left[\left(1-r_{A}\right) \Delta_{31}\right] \\
& +8 J_{r} \frac{\varepsilon}{r_{A}\left(1-r_{A}\right)}\left[-2 s_{13}^{2} \frac{r_{A}}{\left(1-r_{A}\right)^{2}}+\left(c_{12}^{2}-s_{12}^{2}\right) \frac{\varepsilon}{r_{A}}+s_{12}^{2} \frac{\varepsilon r_{A}}{1-r_{A}}\right] \\
& \times \cos \left(\delta+\Delta_{31}\right) \sin \left(r_{A} \Delta_{31}\right) \sin \left[\left(1-r_{A}\right) \Delta_{31}\right] \\
& +16 J_{r} s_{13}^{2} \frac{\varepsilon \Delta_{31}}{\left(1-r_{A}\right)^{2}} \cos \left(\delta+\Delta_{31}\right) \sin \left(r_{A} \Delta_{31}\right) \cos \left[\left(1-r_{A}\right) \Delta_{31}\right] \\
& -8 J_{r} s_{12}^{2} \frac{\varepsilon^{2} \Delta_{31}}{r_{A}\left(1-r_{A}\right)} \cos \left(\delta+r_{A} \Delta_{31}\right) \sin \left(r_{A} \Delta_{31}\right) \\
& -8 J_{r} c_{12}^{2} \frac{\varepsilon^{2} \Delta_{31}}{r_{A}\left(1-r_{A}\right)} \cos \left[\delta+\left(1+r_{A}\right) \Delta_{31}\right] \sin \left[\left(1-r_{A}\right) \Delta_{31}\right] \\
& -8 J_{r} \frac{\varepsilon \Delta_{31}}{r_{A}\left(1-r_{A}\right)}\left(s_{13}^{2} \frac{r_{A}}{1-r_{A}}-s_{12}^{2} \varepsilon\right) \\
& \times \cos \left[\delta+\left(1-r_{A}\right) \Delta_{31}\right] \sin \left[\left(1-r_{A}\right) \Delta_{31}\right]
\end{aligned}
$$

We will consider the precision and speed both of the expression through second order $\left(\mathrm{AM}^{2}\right.$ hereafter $)$ and through $5 / 2$ order $\left(\mathrm{AM}^{5 / 2}\right.$ hereafter $)$.

\footnotetext{
${ }^{11}$ In Ref. $34 r_{\Delta}=\varepsilon$ and $\Delta=2 \Delta_{31} / L$ are used.
} 


\subsubsection{Exact expressions}

For completeness, we discuss two different means of exactly calculating the oscillation probabilities. The first uses the analytic solution to a cubic equation and the second involves numerically diagonalizing the Hamiltonian. We have verified that these are equivalent up to numerical precision of order $\sim 10^{-13}$.

Several pieces of software designed to solve neutrino oscillations in matter also exist in the literature $28,58,59$. While these may offer modest improvements in speed over off-the-shelf linear algebra packages, we have verified that they still do not compete with analytic expressions in terms of speed. In fact, several of them are the same, in part or in full, as the exact expression to be introduced in the next section. NuSquids [59] is a bit different than the other options in that, once it has solved the differential equation for a given matter profile and baseline, extracting the probability (or the flux more generally) for a given energy is fairly efficient.

The ZS expression (1988) It is possible to solve the characteristic polynomial of the Hamiltonian in matter directly. Using this approach one can express the eigenvalues in matter in terms of the vacuum parameters which involves solving a completely general cubic equation 12 . This was done in Ref. [31] (by Zaglauer and Schwarzer, ZS hereafter), where the authors obtain

$$
\begin{aligned}
& {\widehat{m^{2}}}_{1}=\frac{A}{3}-\frac{\sqrt{A^{2}-3 B} S}{3}-\frac{\sqrt{3} \sqrt{A^{2}-3 B} \sqrt{1-S^{2}}}{3}, \\
& \widehat{m}^{2}{ }_{2}=\frac{A}{3}-\frac{\sqrt{A^{2}-3 B} S}{3}+\frac{\sqrt{3} \sqrt{A^{2}-3 B} \sqrt{1-S^{2}}}{3}, \\
& {\widehat{m^{2}}}_{3}=\frac{A}{3}+\frac{2 \sqrt{A^{2}-3 B} S}{3} .
\end{aligned}
$$

The mass splittings in matter are then given by

$$
\begin{aligned}
& {\widehat{m^{2}}}_{21}=\frac{2 \sqrt{3}}{3} \sqrt{A^{2}-3 B} \sqrt{1-S^{2}} \\
& {\widehat{m^{2}}}_{31}=\sqrt{A^{2}-3 B} S+\frac{\sqrt{3}}{3} \sqrt{A^{2}-3 B} \sqrt{1-S^{2}}
\end{aligned}
$$

\footnotetext{
${ }^{12}$ The original solution of the cubic equation was from 60 based on work by Scipione del Ferro and Niccolò Fontana Tartaglia in the sixteenth century.
} 
where $\widehat{x}$ refers to the given quantity in matter and 13

$$
\begin{aligned}
& A=\Delta m_{21}^{2}+\Delta m_{31}^{2}+a, \\
& B=\Delta m_{21}^{2} \Delta m_{31}^{2}+a\left[\Delta m_{31}^{2} c_{13}^{2}+\Delta m_{21}^{2}\left(c_{13}^{2} c_{12}^{2}+s_{13}^{2}\right)\right], \\
& C=a \Delta m_{21}^{2} \Delta m_{31}^{2} c_{13}^{2} c_{12}^{2}, \\
& S=\cos \left\{\frac{1}{3} \arccos \left[\frac{2 A^{3}-9 A B+27 C}{2\left(A^{2}-3 B\right)^{3 / 2}}\right]\right\} .
\end{aligned}
$$

The mixing angles and the CP-phase are

$$
\begin{aligned}
s_{\widehat{12}}^{2} & =\frac{-\left[\left({\widehat{m^{2}}}_{2}\right)^{2}-\alpha{\widehat{m^{2}}}_{2}+\beta\right] \Delta{\widehat{m^{2}}}_{31}}{\Delta{\widehat{m^{2}}}_{32}\left[\left({\widehat{m^{2}}}_{1}\right)^{2}-\alpha{\widehat{m^{2}}}_{1}+\beta\right]-\Delta{\widehat{m^{2}}}_{31}\left[\left({\widehat{m^{2}}}_{2}\right)^{2}-\alpha{\widehat{m^{2}}}_{2}+\beta\right]}, \\
s_{\widehat{13}}^{2} & =\frac{\left(\widehat{m}^{2}{ }^{2}\right)^{2}-\alpha{\widehat{m^{2}}}_{3}+\beta}{\Delta \widehat{m}^{2}{ }_{31} \Delta{\widehat{m^{2}}}_{32}}, \\
s_{\widehat{23}}^{2} & =\frac{E^{2} s_{23}^{2}+F^{2} c_{23}^{2}+2 E F c_{23} s_{23} \cos \delta}{E^{2}+F^{2}}, \\
e^{-i \widehat{\delta}} & =\frac{\left(E^{2} e^{-i \delta}-F^{2} e^{i \delta}\right) c_{23} s_{23}+E F\left(c_{23}^{2}-s_{23}^{2}\right)}{\sqrt{\left(E^{2} s_{23}^{2}+F^{2} c_{23}^{2}+2 E F c_{23} s_{23} \cos \delta\right)\left(E^{2} c_{23}^{2}+F^{2} s_{23}^{2}-2 E F c_{23} s_{23} \cos \delta\right)}},
\end{aligned}
$$

where

$$
\begin{aligned}
\alpha & =\Delta m_{31}^{2} c_{13}^{2}+\Delta m_{21}^{2}\left(c_{13}^{2} c_{12}^{2}+s_{13}^{2}\right), \\
\beta & =\Delta m_{21}^{2} \Delta m_{31}^{2} c_{13}^{2} c_{12}^{2} \\
E & =\left[\Delta m_{31}^{2}\left(\widehat{m}^{2}{ }_{3}-\Delta m_{21}^{2}\right)-\Delta m_{21}^{2}\left(\widehat{m^{2}}{ }_{3}-\Delta m_{31}^{2}\right) s_{12}^{2}\right] c_{13} s_{13}, \\
F & =\Delta m_{21}^{2}\left(\widehat{m}^{2}{ }_{3}-\Delta m_{31}^{2}\right) c_{12} s_{12} c_{13} .
\end{aligned}
$$

As in the case of DMP, the oscillation probabilities in matter can now be obtained by simply replacing the vacuum parameters with the matter parameters in the vacuum oscillation probability from Eq. 1.42 .

\footnotetext{
${ }^{13}$ Note, that in Ref. 31 $D=a$ and there are two typos: the root in the denominator of $S$ should be to the $3 / 2$ power not $1 / 3$ and the numerator of $e^{-\widehat{i \delta}}$ should have a factor of $s_{23} c_{23}$ instead of $s_{23} c_{23}^{2}$.
} 
Numerical diagonalization It is also possible to diagonalize the Hamiltonian numerically. The Schrödinger equation in matter can be written in matrix form as in Eqs. 1.29 and (1.30). In principle, this equation can be solved easily by writing $\mathcal{H}_{\mathrm{F}}=R D R^{\dagger}$, where $D=\operatorname{diag}\left(d_{1}, d_{2}, d_{3}\right)$ is the diagonal matrix containing the eigenvalues $d_{i}$ of $\mathcal{H}_{\mathrm{F}}$, and $R$ is the diagonalization matrix of eigenvectors. Then, the solution is easily found to be

$$
\Psi_{\alpha}(t)=R \operatorname{diag}\left(e^{-i L d_{1}}, e^{-i L d_{2}}, e^{-i L d_{3}}\right) R^{\dagger} \Psi_{\alpha}(0) .
$$

This diaginalization can be performed numerically using for example the packages Eigen [61] or HEigensystem [62,63, among others. We will refer to this method as Diag from here on.

\subsubsection{Comparison of expansions}

We now compare the usefulness of each expression. First, we describe the behavior of the various expressions under several useful limits. Next, we define two metrics: precision and speed/simplicity. Precision can be quantified as either the error or fractional error between a given expression and the exact expression. We focus on fractional error since that is more relevant for experiments although it can become misleading when the probability is small or goes to zero, such as in the high energy limit. While the simplicity of an expression is a somewhat subjective metric, the computational efficiency is somewhat more scientific and quantitative.

\subsubsection{Expansion term}

Each approximate expression is an expansion in one or more parameters. In order to clearly show how each expression behaves, Tab. 1.3 shows which parameters each formula is expanded in. In order to qualify as an expansion parameter, we require that the probability recovers the exact (to all orders) expression as that parameter goes to zero. That is, $x$ is an expansion parameter if and only if

$$
\lim _{x \rightarrow 0} P_{\text {approx }}(x)=P_{\text {exact }}(x=0) .
$$

We note that, as many expressions drop higher order terms of more than one parameter at a time, it is quite common for expressions to not be true expansions in the sense of Eq. (1.57) in that all of the parameters that were treated as small numbers simultaneously. 


\begin{tabular}{|l|c|c|c|}
\hline & $\epsilon(\varepsilon)$ & $s_{13}$ & $a / \Delta m_{31}^{2}$ \\
\hline Madrid(like) & $\times$ & $\times$ & $\times$ \\
AKT & $\checkmark$ & $\checkmark$ & $\checkmark$ \\
MP & $\checkmark$ & $\times$ & $\times$ \\
DMP & $\checkmark$ & $\checkmark$ & $\checkmark$ \\
AKS & $\times$ & $\times$ & $\times$ \\
MF & $\checkmark$ & $\times$ & $\times$ \\
AJLOS(48) & $\checkmark$ & $\times$ & $\times$ \\
AM & $\times$ & $\times$ & $\times$ \\
\hline
\end{tabular}

Table 1.3: The expansion terms of each expression. Terms that are expansion parameters in the sense of Eq. (1.57) are denoted with a green check $(\checkmark)$, while terms that are not are denoted with a red cross $(\times)$. Note that Madrid refers also to the expression AJLOS(31) and FL which are all generally quite similar. $\mathrm{AM}$ refers to both $\mathrm{AM}^{2}$ and $\mathrm{AM}^{5 / 2}$, and DMP refers to both $\mathrm{DMP}^{0}$ and $\mathrm{DMP}^{1}$.

We find that DMP (at any order) as well as AKT are the only expressions that are an expansion in $s_{13}$ and the matter potential. Also, while several expressions are expansions in $\epsilon$ or $\varepsilon$ (including DMP and AKT), some are not an expansion in $\epsilon$ or $\varepsilon$ either despite treating $\varepsilon$ as a smallness parameter, such as the Madridlike expressions. In addition to the parameters listed in the table, we note that none of the expressions are exact as $L \rightarrow 0$, the so-called vacuum mimicking regime 64.

\subsubsection{Precision and speed}

In this section we compare the different expressions in terms of precision and speed. As a benchmark point we use the standard oscillation parameters in Tab. 1.1. We take $\delta$ to be slightly off-maximal to avoid any unintentional cancellations and to require both $\sin \delta$ and $\cos \delta$ terms to be correct. We choose as benchmark baseline $L=1300 \mathrm{~km}$ and density $\rho=3 \mathrm{~g} / \mathrm{cm}^{3}$, the configuration for the DUNE experiment 65 67 although our results are applicable to any current or future long-baseline experiment, including those focusing on the second oscillation maximum.

The probabilities for selected expressions are shown in Fig. 1.3 for both neutrinos (left) and antineutrinos (right). They should be compared to the exact curves given by ZS. To compare the precision of the formulas in a more quantitative way, we show $\frac{\left|P_{\text {test }}-P\right|}{P}=\frac{|\Delta P|}{P}$, where $P=P_{\mathrm{ZS}}$ is the exact formula. The result is shown in Fig. 1.4. As one can see, for low energies DMP gives the best 


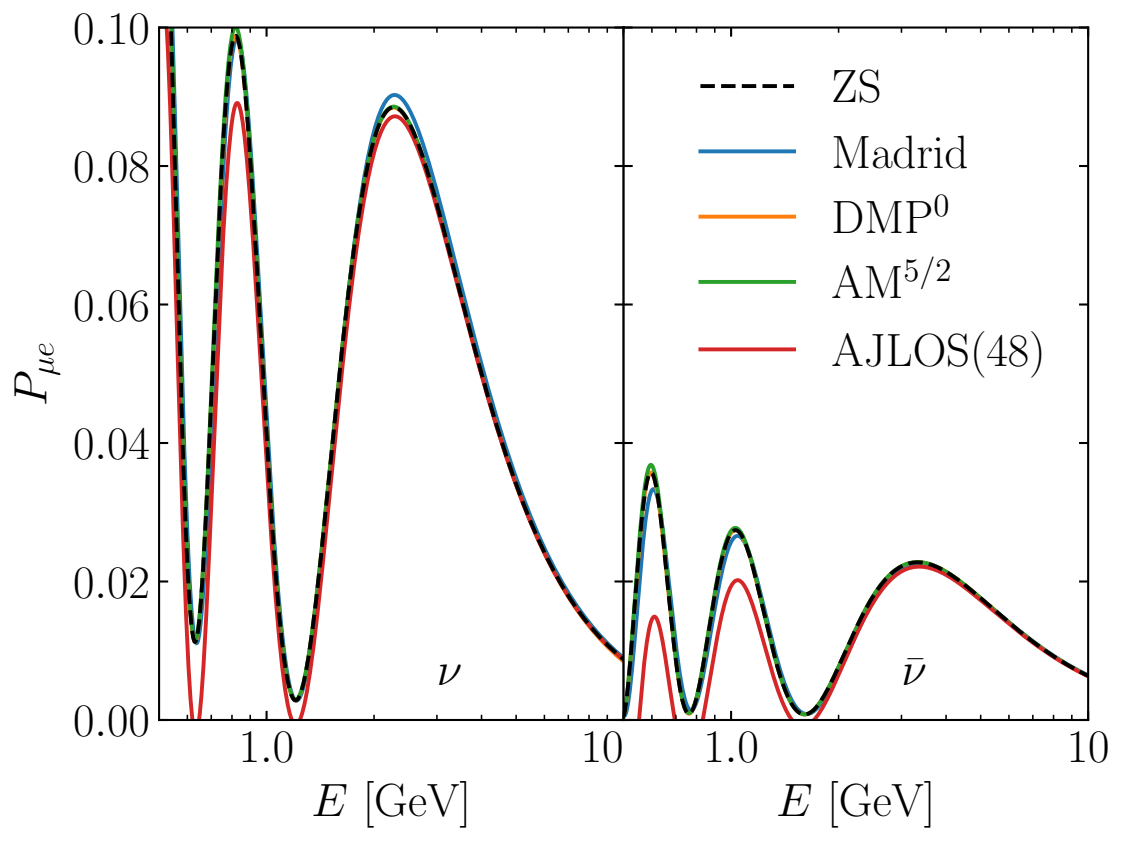

Figure 1.3: Various expressions for the oscillation probabilities for neutrinos (left) and antineutrinos (right) at $L=1300 \mathrm{~km}$. The exact expression (ZS) is shown as a black dashed curve. Figure taken from Ref. 1. 


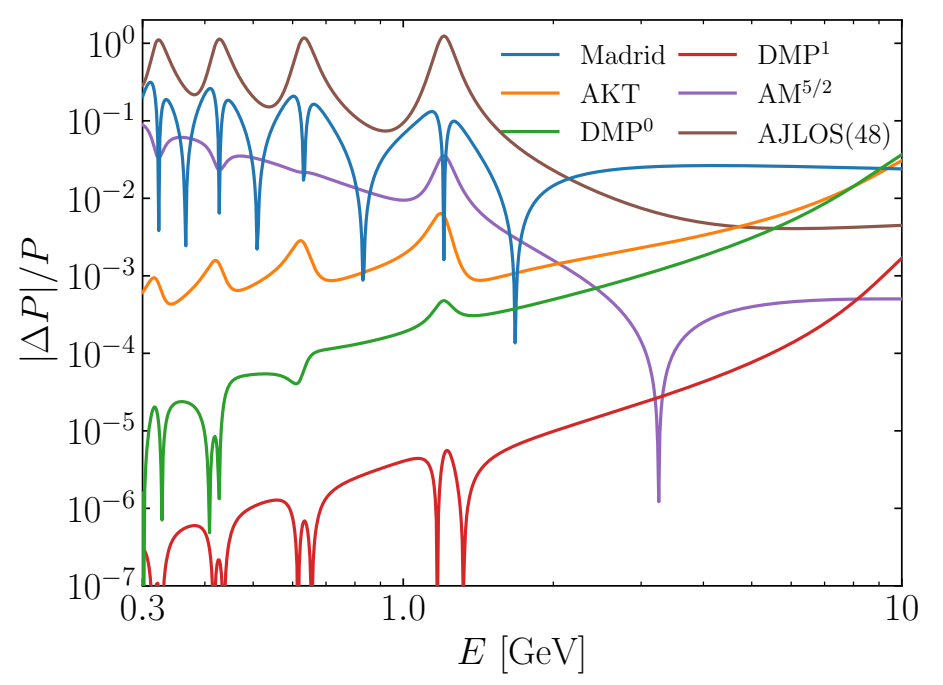

Figure 1.4: The relative precision in the $\nu_{\mu} \rightarrow \nu_{e}$ channel of several of the expressions compared to the exact expression. The various sharp dips down are where the approximate and exact solutions cross. Note that towards lower energies all the expressions tend to do poorly except DMP. At high energies $|\Delta P| / P=\left|P_{\text {test }}-P\right| / P$ with $P=P_{\mathrm{ZS}}$ becomes somewhat misleading as $P \rightarrow 0$, but the precision of each formula levels out (for $\mathrm{DMP}^{0}$ and $\mathrm{AKT}\left(\mathrm{DMP}^{1}\right)$ it levels out at $\Delta P / P=0.057(0.007)$ past the atmospheric resonance). Figure taken from Ref. 1].

results, while for $E>2 \mathrm{GeV}$ the most precise result is $\mathrm{AM}^{5 / 2}$. The precision of $\mathrm{DMP}^{0}$ and $\mathrm{AKT}$ is the worst at the atmospheric resonance $(\sim 11 \mathrm{GeV})$ before leveling off, although the probability is approaching zero thus this region is less relevant experimentally. We also show the precision of the several Madrid-like expressions in Fig. 1.5. The precision of the remaining expressions can be found in Fig. 1.6. Note that the sharp dips are not representative of improved precision, rather they represent a crossing between the exact and approximate expressions. In addition, the peaks in the errors appear when the oscillation probability, and thus the denominator, goes to zero.

In order to more clearly compare the precision of each expression, we show in Fig. 1.7 the precision with which each expression reconstructs the first and second oscillation maxima. We focus on the oscillation maxima because the heights (probability) of the maxima are an important test for $\mathrm{CP}$ violation 68 and the locations (energy) of the maxima are an important test for the atmospheric mass 


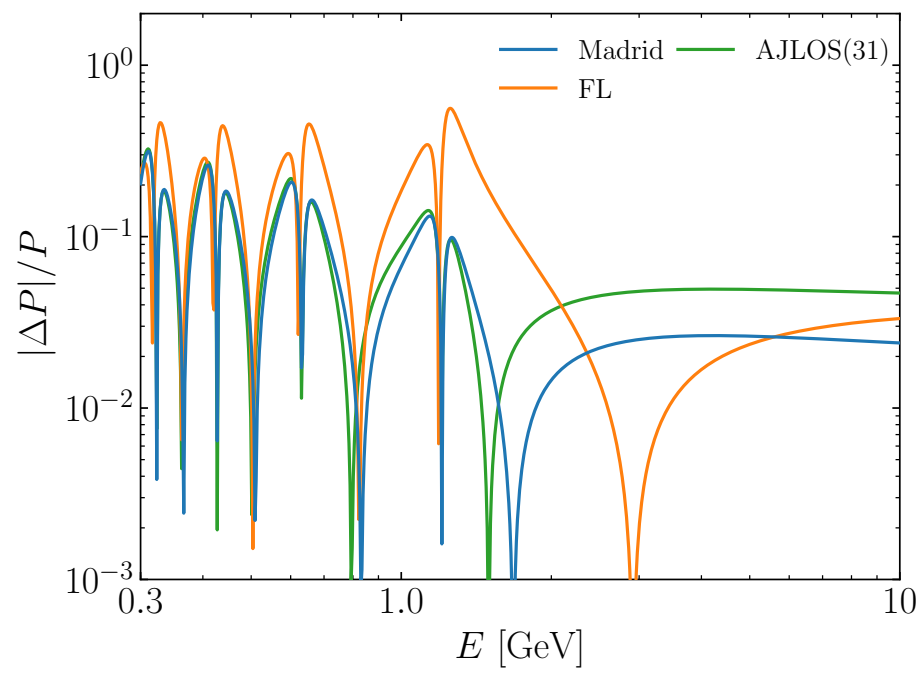

Figure 1.5: The precision of the Madrid-like expressions. Figure taken from Ref. [1.

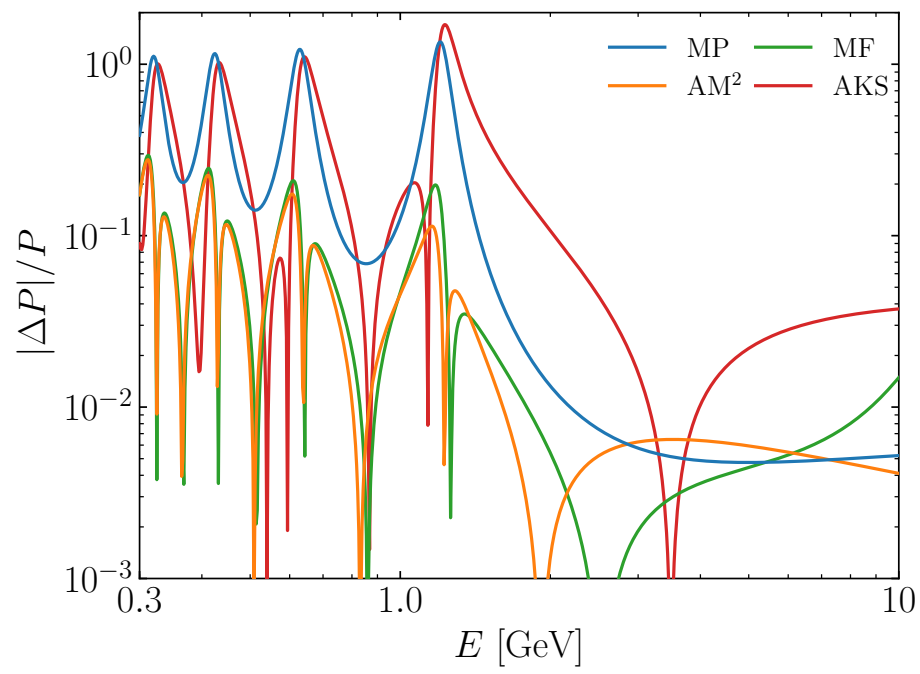

Figure 1.6: The precision of the remaining expressions. Figure taken from Ref. [1]. 


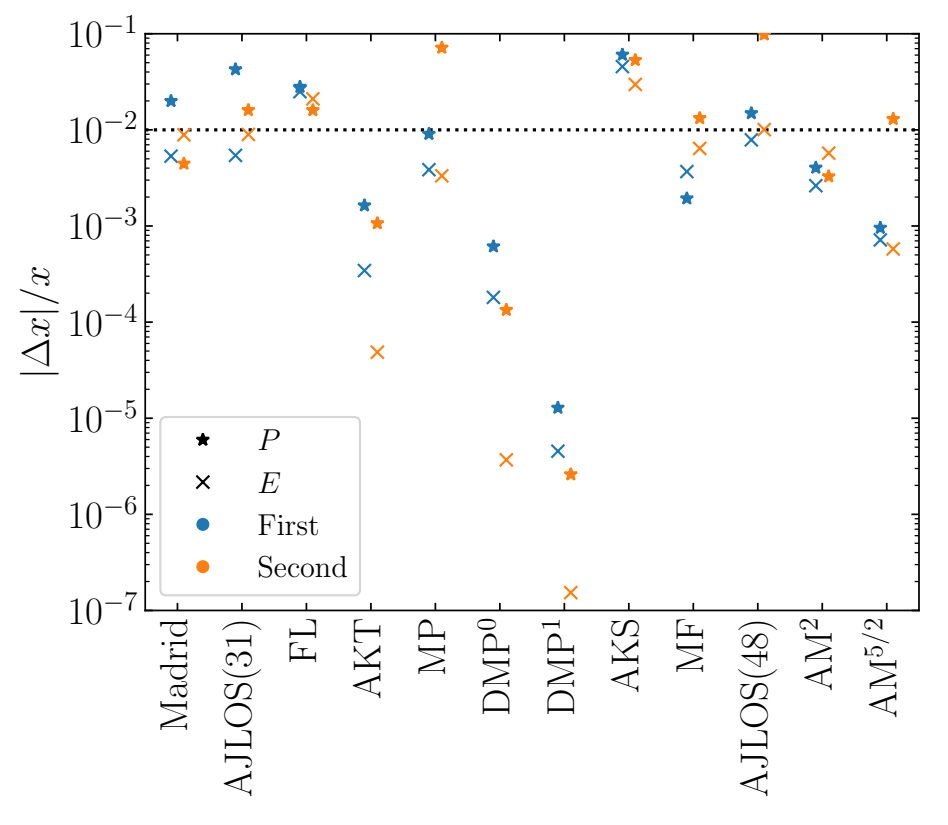

Figure 1.7: The relative error at the first (blue, left) and second (orange, right) oscillation maxima in the probability (star) and energy (cross) for each formula. While evaluating these on the peaks could lead to a chance conspiracy, we have verified that the results are fairly robust under changes in $\delta$ and in energy away from the maxima. Figure taken from Ref. [1.

splitting [69. The horizontal line at $1 \%$ is to guide the eye. Since DUNE and other next generation long-baseline experiments are aiming to reach near the percent level in precision, we cannot introduce theoretical errors larger than $1 \%$. We have also verified that these results are generally robust under changes in the oscillation parameters, although for certain specific values of say the CPviolating phase some of the fairly precise expressions may appear to perform much better if there is a crossing between the approximate and exact expressions at the oscillation maximum.

We also measured the computational time on a single core i7 processor using $\mathrm{c}++$ with the gnu v7.3.0 compiler ${ }^{14}$. Our result can be found in Fig. 1.8 along with the precision at the first oscillation maximum. The green (red) dots are those for which the probability at the first maximum is better (worse) than $1 \%$.

\footnotetext{
${ }^{14}$ We have performed the same test using Fortran with the gfortran compiler, obtaining qualitatively the same results.
} 


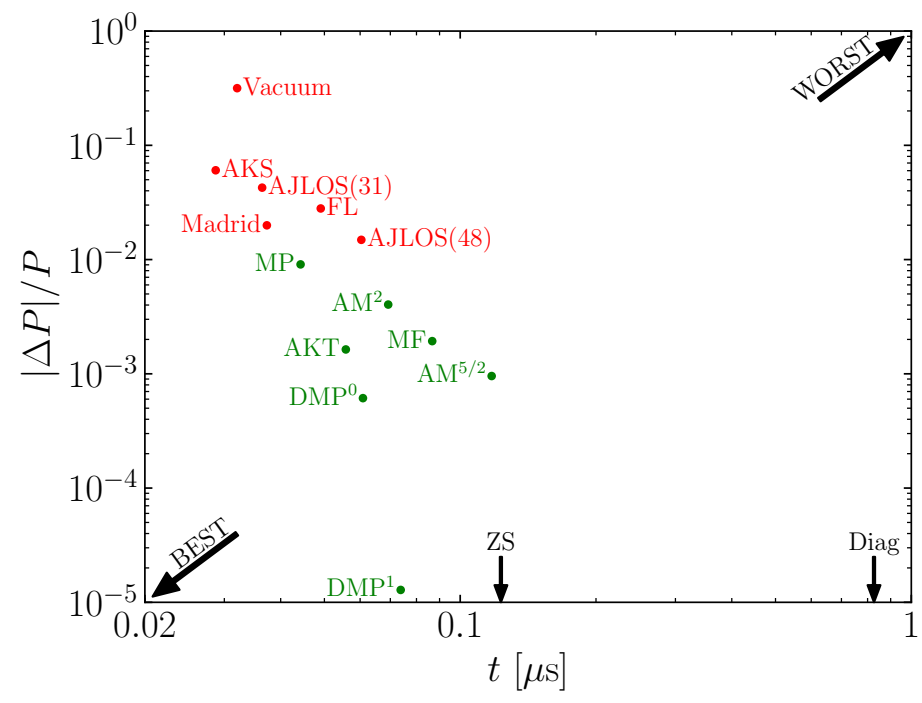

Figure 1.8: Precision of each expression compared with the computational speed. The vacuum dot refers to calculating the exact vacuum expression without any matter effects. The precision is defined as a the relative error in probability at the first maximum and the speed is the time it takes to compute one probability. Figure taken from Ref. [1]. 
We also measure the computational time for the exact vacuum expression from Eq. 1.42 for comparison. All of the approximate expressions and ZS are faster than the diagonalization by about an order of magnitude or more, depending on the expansion of interest. The DMP expressions have the best precision among the approximate expressions by a considerable amount.

When computational speed is the primary, if $1 \%$ precision is required then MP is the best expression, although it is right at $\sim 1 \%$ and has much larger errors at $\sim 1 \mathrm{GeV}$. After MP, the next simplest expression that is also better than $1 \%$ is $\mathrm{DMP}^{0}$ which is precise at the per mille level or better.

\subsection{Final remarks}

If neutrinos travel through a non constant matter profile, the techniques discussed in the last section do not work anymore. This happens, for example, for neutrinos produced in the Earth's atmosphere which travel through all of the Earth interior before being detected or, in the case of solar neutrinos. These neutrinos are produced in the interior of the Sun, where matter effects are important and the matter profile cannot be assumed constant. Next, they travel through the vacuum between Sun and Earth and, finally, again through matter when going through the Earth interior. However, the neutrino oscillation probability can still be calculated by discretizing the path of the neutrino through the medium and then applying iteratively numerical diagonalization as discussed in Sec. 1.3.1.4.

Along this thesis, we will not focus only on standard 3-neutrino oscillations, but will also discuss several extensions of the standard model, where the 3 neutrino paradigm is extended with several new oscillation parameters. Note that most of the current experimental results point towards three standard neutrinos. However, a few extensions are theoretically very well motivated as, for example, non-standard neutrino interactions which can appear at sub-leading orders in the neutrino oscillation probabilities and are not accessible with current and possibly neither with future experiments. Other extensions are motivated by experimental observations such as eV-scale sterile neutrinos. Whenever we depart from the standard picture, it is more convenient to simply make use of numerical tools than derive exact or approximate solutions of the Schrödinger equation. 


\section{Chapter 2}

\section{Neutrino oscillation parameters}

In the last chapter we have seen how to describe neutrino oscillations. In this chapter, we focus on how to extract the values of the oscillation parameters from the analysis of experimental data. To extract the oscillation parameters we have to perform a statistical analysis of the experimental data and can then obtain allowed intervals for the parameters at different confidence levels. The important quantity is, in this case, the expected number of events in a given experiment for a given set of values of the oscillation parameters $\vec{p}, N^{\exp }(\vec{p}, \vec{\alpha})$. The expected number of events also depends on systematic uncertainties $\vec{\alpha}$ related to unknowns, other than the oscillation parameters, which can be very different depending on the type of experiment. This quantity is then compared $\sqrt{1}$ with the observed number of events $N^{\text {dat }}$ by calculating the $\chi^{2}$ function in terms of the oscillation parameters. In general, we have

$$
\chi^{2}(\vec{p})=\min _{\vec{\alpha}}\left\{\sum_{n} \frac{\left[N^{\text {dat }}-N^{\exp }(\vec{p}, \vec{\alpha})\right]^{2}}{\sigma^{2}}+\chi_{\text {sys }}^{2}(\vec{\alpha})\right\}
$$

if we assume Gaussian statistics, or

$$
\chi^{2}(\vec{p})=\min _{\vec{\alpha}}\left\{2 \sum_{n}\left(N^{\exp }-N^{\mathrm{dat}}+N^{\mathrm{dat}} \log \frac{N^{\mathrm{dat}}}{N^{\exp }(\vec{p}, \vec{\alpha})}\right)+\chi_{\text {sys }}^{2}(\vec{\alpha})\right\}
$$

\footnotetext{
${ }^{1}$ Along this thesis, we will always use $\chi^{2}$ distributions.
} 
assuming Poisson statistics. Here $\chi_{\text {sys }}^{2}$ contains penalty terms in the treatment of systematic uncertainties. Usually, these uncertainties are introduced as nuisance parameters with Gaussian priors, as

$$
\chi_{\text {sys }}^{2}(\vec{\alpha})=\sum_{j}\left(\frac{\alpha_{j}-\mu_{j}}{\sigma_{j}}\right)^{2},
$$

where $\mu_{j}$ and $\sigma_{j}$ are the expectation value and the standard deviation for the parameter $\alpha_{j}$. After calculating $\chi_{k}^{2}(\vec{p})$ for each experiment $k$ we include in our analysis, we define the combined $\chi^{2}$ function as

$$
\chi_{\text {comb }}^{2}(\vec{p})=\sum_{k} \chi_{k}^{2}(\vec{p}) .
$$

When plotting our results, we obtain one or two-dimensional projections from this function by marginalizing over all parameters except the ones of interest,

$$
\chi^{2}(x, y)=\min _{\vec{p} \backslash\{x, y\}}\left\{\chi_{\text {comb }}^{2}(\vec{p})\right\} .
$$

Note that here "comb" can refer to the global combination of all experiments, but also to some subset. In a very simplified form, the expected number of events of a flavor $\beta$ can be written as

$$
N_{\beta}^{\exp } \sim \sum_{\gamma=e, \mu, \tau} \phi_{\gamma} P_{\gamma \beta} R_{\beta} .
$$

Here $\phi_{\gamma}$ is the flux of neutrinos of an initial flavor $\gamma, P_{\gamma \beta}$ is the probability to oscillate into the flavor $\beta$ in the trajectory from the production point to the neutrino detector and $R_{\beta}$ is the detector response to detect a neutrino of flavor $\beta$ that contains the relevant cross sections, efficiencies and the event reconstruction. The oscillation probability is the common factor in all experiments, while the fluxes and detector responses are individual to each of the experiments. Of course, this equation is oversimplified, because each of these quantities depends itself on other quantities. For example, all three depend on the neutrino energy, E. Note that both, the flux and the detector response, can be modified by systematic uncertainties which have to be included in the analyses.

We differentiate neutrino oscillation experiments by looking at the way the neutrinos are produced. In general we distinguish four different types of experiments: 
- Solar neutrino experiments (SOL) observe neutrinos which are created in the interior of the Sun.

- Atmospheric neutrino experiments (ATM) observe neutrinos which are created in the atmosphere of our planet.

- Reactor neutrino experiments (REAC) observe neutrinos created in nuclear processes in the reactors of nuclear power plants.

- Long baseline accelerator neutrino experiments (ACC) observe neutrino created in particle accelerators.

Note that we use both, natural sources such as the Sun and the Earth's atmosphere, and artificial sources as reactors and accelerators, to measure neutrino properties. Each type of experiment is sensitive to different parameters. The main sensitivities are indicated in Tab. 2.1. Here LBL and SBL stand for long baseline and short baseline 2 , respectively. Since many of the parameters are measured by several classes of experiments, a combined, or global, fit of all data will give more precise results than a measurement of a single experiment on its own. This way the advantages of every type of experiment are used. This global analysis is exactly the purpose of this chapter. First, we will go into more detail describing each type of experiment. Next, we will discuss the synergies among different types of experiments to obtain an improved determination of the neutrino oscillation parameters.

A former version of the global fit is summarized in Appendix A while preliminary results of a new analysis including the most recent neutrino oscillation data, which is not finished yet, are presented in Appendix B. This chapter describes the results published in Ref. [2], which have been presented in numerous conferences, seminars and workshops and can be regarded as one of the main results of this thesis.

\subsection{Experiments included in the global fit}

In this section, we summarize all of the experimental data included in the global fit to neutrino oscillations. Note that all analyses are performed within the three-neutrino framework. If some experiments are not sensitive to a set of oscillation parameters, we fix them to their best fit values obtained in a different

\footnotetext{
${ }^{2}$ We use the term short baseline for baselines of the order of $\sim 1 \mathrm{~km}$. We will not discuss results from the search of light sterile neutrinos here.
} 


\begin{tabular}{|l|c|c|}
\hline Parameter & Main contribution from & Other contributions from \\
\hline$\Delta m_{21}^{2}$ & KamLAND & SOL \\
$\left|\Delta m_{31}^{2}\right|$ & ACC+ATM+REAC & - \\
$\theta_{12}$ & SOL & KamLAND \\
$\theta_{23}$ & ACC+ATM & - \\
$\theta_{13}$ & REAC & ACC+SK+KamLAND \\
$\delta$ & ACC & SK \\
MO & (ACC+REAC) and SK & - \\
\hline
\end{tabular}

Table 2.1: The main contribution to each of the oscillation parameters from the different classes of experiments. Here, ACC refers to all accelerators, REAC to short baseline reactors, ATM to atmospheric neutrino experiments and SOL to solar experiments.

part of the analysis. For example, the experiments discussed in Sec. 2.1.1 are the only ones sensitive to the solar parameters. Therefore, in the rest of the analyses these parameters are fixed to the best fit values obtained in this subsection.

\subsubsection{Solar experiments and KamLAND}

In this section, we review the data used in our global fit from solar neutrino experiments and from the (unique) long-baseline reactor experiment KamLAND.

\section{Solar neutrino experiments}

Solar neutrinos are produced in thermonuclear reactions in the interior of the Sun when burning hydrogen into helium. The main nuclear chains producing neutrinos are the so-called proton-proton $(p p)$ chain and the CNO cycle, see Fig. 2.1. All the reactions which produce neutrinos are shown in this figure. The fluxes for the neutrinos coming from different reactions are shown in Fig. 2.2. Basically, all processes lead to the fusion of four protons into one Helium nucleus

$$
4 p \rightarrow{ }^{4} \mathrm{He}+2 e^{+}+2 \nu_{e}+\gamma .
$$

Our solar oscillation analysis includes data from several solar neutrino oscillation experiments. We use data corresponding to the total rate from the radiochemical experiments Homestake [72, GALLEX/GNO [73] and SAGE [74]. In these experiments, the detector consisted of different solutions containing chlorine 


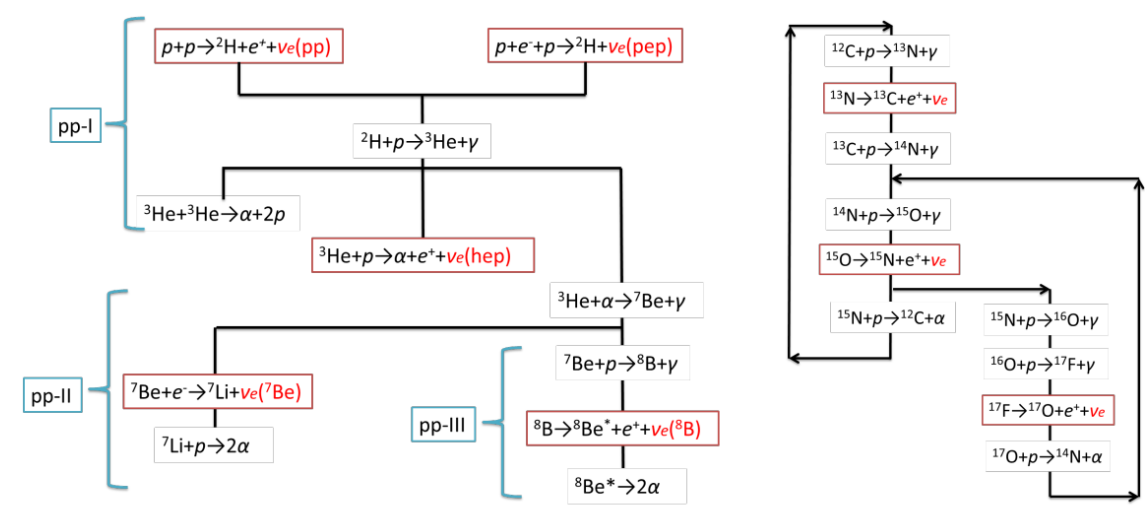

Figure 2.1: A schematic representation of the $p p$ chain (left) and the CNO cycle (right). Figure taken from Ref. 70.

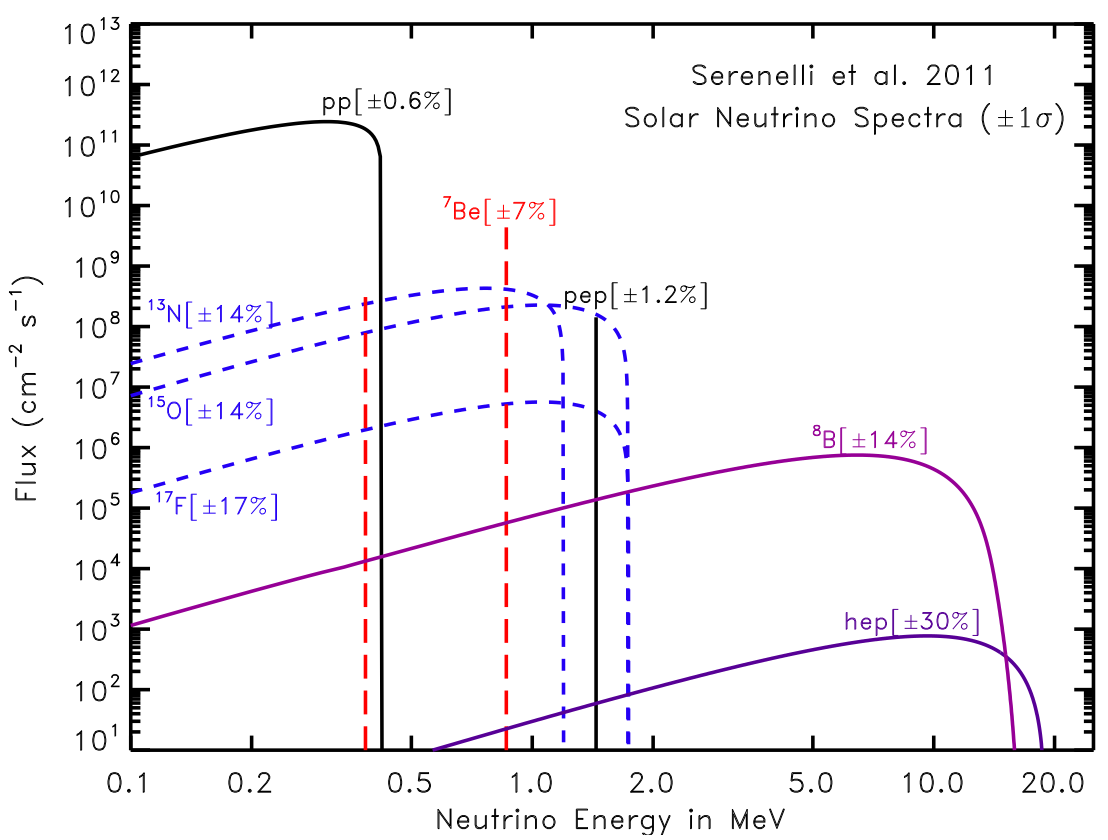

Figure 2.2: The flux of solar neutrino. The lines mean that this type of neutrino is produced mono-energetically. Figure taken from Ref. [71].

(Homestake) and gallium (Gallex/GNO, SAGE). When solar neutrinos interact with the detector material, they produce argon and germanium, 


$$
\begin{gathered}
\nu_{e}+{ }^{37} \mathrm{Cl} \rightarrow{ }^{37} \mathrm{Ar}+e^{-}, \\
\nu_{e}+{ }^{71} \mathrm{Ga} \rightarrow{ }^{71} \mathrm{Ge}+e^{-} .
\end{gathered}
$$

After some time, the amount of argon or germanium can be counted, hence the name "counting experiments", and the solar flux at Earth can be calculated. Because of this detection technology, neutrinos can not be observed in real time.

We include the energy spectrum from low-energy ${ }^{7}$ Be neutrinos observed by Borexino 75, 76. This experiment uses liquid scintillator as detection material and observes neutrinos via quasi-elastic scattering on electrons

$$
\nu+e^{-} \rightarrow \nu+e^{-}
$$

allowing for a real-time detection. The same reaction is used to detect neutrinos at the water cherenkov detector Super-Kamiokande, which uses 50 kton of ultra pure water as detection material. We include data from Super-Kamiokande phases IIV $70,77,79$. For phase I we use the zenith angle spectrum and for the remaining phases the day/night spectrum.

Finally, we include the day/night spectrum of the three SNO phases 80,81. In this experiment, heavy water was used as detection material. This allowed the experiment to measure neutrinos, apart from quasi-elastic scattering on electrons, via charged current interactions with deuterium

$$
\nu_{e}+d \rightarrow p+p+e^{-}
$$

and neutral current interactions with deuterium

$$
\nu_{\alpha}+d \rightarrow \nu_{\alpha}+n+p
$$

Using this last reaction, it was possible to show that the solar neutrino flux was indeed conserved, but the initial electron neutrinos had oscillated into all different flavors when arriving at the detector and, hence, solve the solar neutrino problem.

When neutrinos travel through the Earth, they feel matter effects as explained in Chapter 1. Therefore, we expect an asymmetry for the neutrino flux measured during night (traversing the Earth) with respect to the one measured during the day. This day/night asymmetry is defined in terms of the day/night fluxes as

$$
A_{\mathrm{DN}}=2 \frac{\phi_{D}-\phi_{N}}{\phi_{D}+\phi_{N}}
$$


The measured asymmetry obtained by Super-Kamiokande at phases I-IV $\mid 70$ is given by

$$
A_{\mathrm{DN}}=[-3.3 \pm 1.1(\text { stat }) \pm 0.8(\text { syst })] \% .
$$

This result is $2.4 \sigma$ away from zero, providing an indirect indication for matterenhanced neutrino oscillations inside the Earth. The result of our combined analysis of solar neutrino oscillation data is shown in Fig. 2.3 .

\section{KamLAND}

Nuclear reactor core generate energy through the neutron-induced nuclear fission. While research reactors use fuel highly enriched with ${ }^{235} \mathrm{U}$, commercial reactors contain more fissile isotopes. Especially, many neutrons are captured by ${ }^{238} \mathrm{U}$ which then produces itself the new fissile isotopes ${ }^{241} \mathrm{Pu}$ and ${ }^{239} \mathrm{Pu}$. Reactor neutrinos are then produced through the beta-decay of the daughters of these four isotopes. In this process, a neutron is converted into a proton, producing an electron and an electron antineutrino

$$
n \rightarrow p+e^{-}+\bar{\nu}_{e} .
$$

In general, an average of 6 neutrinos are produced per fission, leading to approximately $2 \times 10^{20} \bar{\nu}_{e}$ emitted isotropically every second for each $\mathrm{GW}_{\text {th }}$ of thermal power of the nuclear core. Roughly $30 \%$ of the neutrinos are created with energies above $1.8 \mathrm{MeV}$, which is the necessary energy to observe them via inverse beta-decay

$$
\bar{\nu}_{e}+p \rightarrow e^{+}+n .
$$

The long-baseline reactor neutrino experiment KamLAND used a single detector measuring neutrinos from 56 Japanese nuclear power plants with an average distance of $180 \mathrm{~km}$. The long distance made KamLAND sensitive to the solar oscillation parameters. In our global fit, we include the final KamLAND data set 82 . The result of our analysis is plotted together with the result from the analysis of solar neutrino oscillation data in Fig. 2.3

\subsubsection{Short-baseline reactor experiments}

Apart from KamLAND, there are several more reactor neutrino experiments. Unlike KamLAND, however, they are located typically at around $1 \mathrm{~km}$ distance to the nuclear power plant. This makes them sensitive to measure $\theta_{13}$ and $\Delta m_{e e}^{2}=$ $\cos ^{2} \theta_{12} \Delta m_{31}^{2}+\sin ^{2} \theta_{12} \Delta m_{32}^{2} 41,42$. Using current data from RENO 83 and 


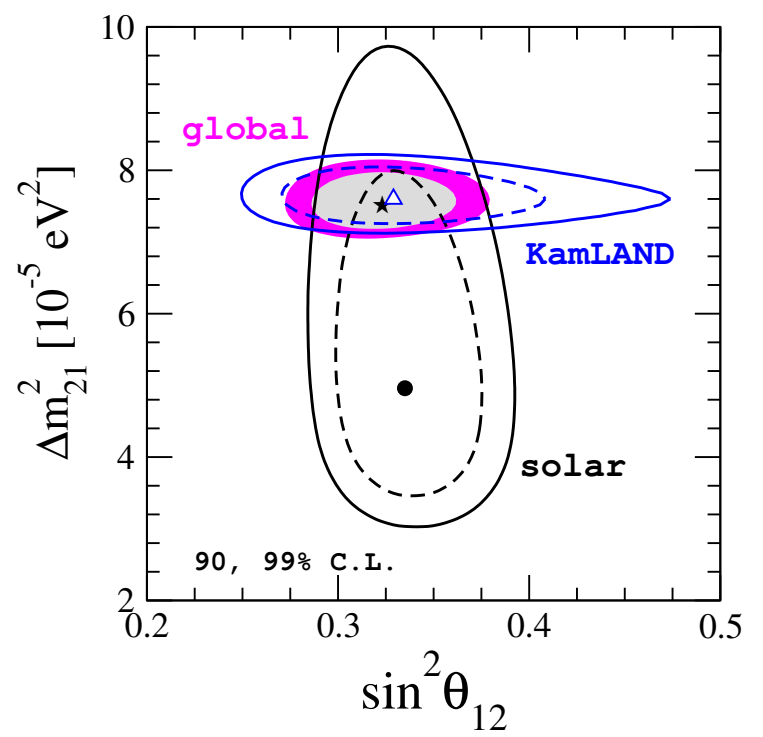

Figure 2.3: 90 and $99 \%$ C.L. (2 d.o.f.) allowed regions at the $\sin ^{2} \theta_{12^{-}} \Delta m_{21}^{2}$ plane after marginalizing over $\theta_{13}$ from the combined solar analysis (black lines), from KamLAND (blue lines) and from the combination of both data sets (colored regions).

Daya Bay [84], it was shown that there is also some sensitivity to bound the solar parameters [85]. Note that these bounds, however, are not competitive with the results coming from KamLAND, so we fix in our analysis the solar parameters to the ones measured by the solar experiments and KamLAND. Here, we use data coming from the reactor experiments Daya Bay [86], RENO [87, 88] and Double Chooz [89], which we will describe with some detail in the following subsections.

\section{Daya Bay}

Daya Bay is a multi-core and multi-detector experiment, with eight 20 ton Gd-doped liquid scintillator antineutrino detectors (ADs) located at three experimental halls (EHs). At EH1 and EH2, two ADs were deployed while the remaining four ADs were assigned to the far site, EH3. The thermal power of each reactor is $2.9 \mathrm{GW}_{\text {th }}$ and the baseline to the near and far sites (EH1 and EH2) are in the range $0.35-0.6 \mathrm{~km}$ and $1.5-1.9 \mathrm{~km}$, respectively. After 1230 days of data taking, Daya Bay has measured approximately two hundred thousand inverse beta decay events at the far site. Thanks to the large statistics and the 
reduction of systematic uncertainties, due to having several functionally identical ADs, Daya Bay has provided the most precise determination of the reactor mixing angle $\theta_{13}$. In this analysis, we have included the antineutrino event energy spectra from the three EHs. Systematic uncertainties accounting for total and detector normalization, as well as core-related systematic uncertainties and energy scale errors were included in the analysis. Systematic uncertainties accounting for the background normalization in each experimental hall have been also included in the analysis, where we have used the background expectations from the ancillary files from Ref. [86. The results of the analysis of Daya Bay data are shown in Fig. 2.4. From this figure it is clear that the analysis of reactor data will be dominated by Daya Bay.

\section{RENO}

The RENO experiment has recently reported 1500 live days of data from antineutrinos produced at six reactor cores each one with $\sim 2.8 \mathrm{GW}_{\text {th }}$ thermal power. The experiment detects neutrinos at a near and at a far detector (each detector with 16 ton of fiducial mass) located at $0.294 \mathrm{~km}$ and $1.383 \mathrm{~km}$ from the line joining the six reactor cores, respectively ${ }^{3}$. Thanks to the improved precision, the spectral fit analysis of RENO data is now sensitive to the neutrino oscillation phase, as reported in Refs. 87, 88. In our analysis, we have considered the near and far detector event energy distribution. We have fitted the measured energy spectrum at each detector after the subtraction of the background, normalizing our simulation to the expected spectra reported by the RENO collaboration. Systematic uncertainties accounting for core-related (0.9\% for each core) and detector uncertainties ( $0.2 \%$ for each detector) [91], have been included in our analysis in the form of nuisance parameters. We have also included a nuisance parameter accounting for the total normalization uncertainty, that has been left completely free in the analysis. The results of our analysis are plotted together with the remaining reactor experiments in Fig. 2.4 .

\section{Double Chooz}

The Double Chooz experiment detects antineutrinos produced at two reactor cores with a $2 \times 4.27 \mathrm{GW}_{\text {th }}$ total thermal power using a near and far detector of 8 ton fiducial mass each, located at $0.4 \mathrm{~km}$ and $1.05 \mathrm{~km}$, respectively. The data set considered in this analysis corresponds to 461 days of data with far detector only (far-I) plus 212 days of far detector data with a near detector (far-II), as

\footnotetext{
${ }^{3}$ The exact detector to reactor distances from Ref. 90 were used in our simulation.
} 

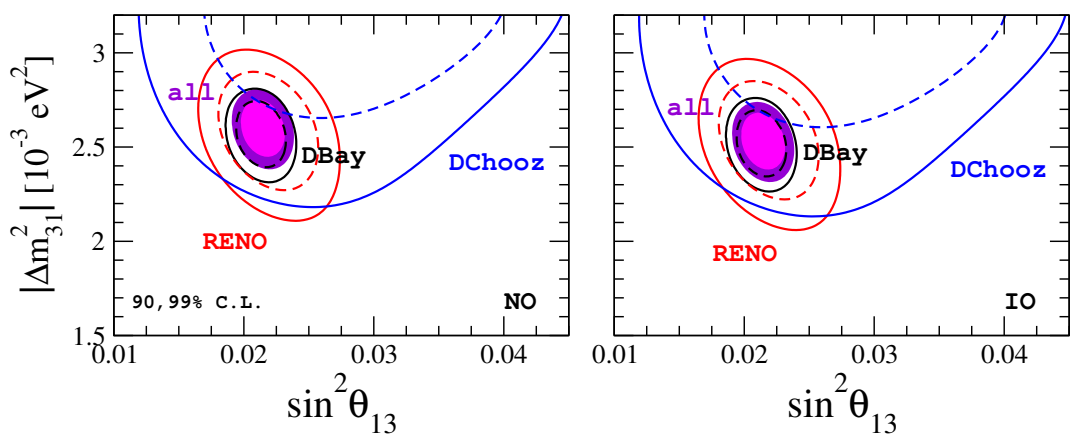

Figure 2.4: 90 and 99\% C.L. (2 d.o.f.) allowed regions at the $\sin ^{2} \theta_{13^{-}} \Delta m_{31}^{2}$ plane from individual reactor neutrino experiments (dashed and solid lines) and from the combination of the three experiments (colored regions). The left (right) panels correspond to normal (inverted) mass ordering. Figure taken from Ref. [2].

reported in Ref. [89]. The event energy spectrum from the far-I and far-II data periods were included in the analysis. Systematic uncertainties considered in our simulation account for the signal and background normalization as well as for the total normalization. The total background has been extracted from the data reported in Ref. 89. The results of the Double Chooz analysis are also shown in Fig. 2.4 .

\subsubsection{Atmospheric experiments}

When cosmic rays collide with particles in the Earth's atmosphere they start a particle shower which eventually creates a lot of neutrinos. The energy of $\nu_{\mu}$ and $\nu_{e}$ (and their antiparticles) produced in the atmosphere can range from a few $\mathrm{MeV}$ up to around $10^{9} \mathrm{GeV}[92$, although only events up to $\sim 100 \mathrm{TeV}$ are currently detectable. However, the energy of neutrinos used to determine oscillation parameters ranges from $\sim 0.1 \mathrm{GeV}$ to $\sim 100 \mathrm{GeV}$. In our global fit, we include data from IceCube DeepCore [93], ANTARES [94] and Super-Kamiokande [95]. Since the largest part of the atmospheric neutrino flux is formed by $\nu_{\mu}$ and $\bar{\nu}_{\mu}$ and, since it is more difficult to identify electrons in the detector, the main channel used in current atmospheric neutrino experiments is $\nu_{\mu} \rightarrow \nu_{\mu}$, which makes them mostly sensitive to $\theta_{23}$ and $\Delta m_{31}^{2}$. Note, however, that the Super-Kamiokande experiment also detected a large sample of electron events from $\nu_{e}$ appearance [95, 96, resulting in a small sensitivity to $\theta_{13}$ and $\delta$. In the following subsections we will describe the analyses performed. 


\section{IceCube DeepCore}

IceCube is a $1 \mathrm{~km}^{3}$ multipurpose neutrino telescope placed near the AmundsenScott South Pole Station, buried beneath the surface and extending up to a depth of about 2500 meters. It uses Cherenkov light to detect high energy neutrinos, using the polar ice as the medium where this light is produced. It has 86 strings with 60 digital optical modules (DOMs) each, placed at a depth that goes from $1450 \mathrm{~m}$ to $2450 \mathrm{~m}$ into the ice. In this analysis we use data from DeepCore, a denser region of strings inside IceCube, designed to measure the atmospheric neutrino flux at low energies. The observed neutrino energy lies between $6.3 \mathrm{GeV}$ and $56.2 \mathrm{GeV}$, way below the energy threshold of IceCube, which is about $100 \mathrm{GeV}$.

In order to determine the atmospheric neutrino oscillation parameters, in this simulation we use data published by IceCube DeepCore in Ref. 93. Neutrino data are presented in 64 bins, with 8 energy-bins and 8 bins in zenith-angle, see 97 . Tables with systematic detector uncertainties, optical efficiencies and uncertainties produced through scattering at holes opened in the ice for the depletion of the DOMs are also provided. The fluxes for atmospheric neutrinos are taken from 98, 99. We perform the numerical integration in matter using the Preliminary Reference Earth Model (PREM) [100.

In Fig. 2.5 we compare the allowed regions in the atmospheric neutrino oscillation parameters $\sin ^{2} \theta_{23}$ and $\Delta m_{31}^{2}$ obtained from DeepCore with the results obtained by the other atmospheric experiments, to be discussed in the following.

\section{The ANTARES neutrino telescope}

ANTARES is a deep sea neutrino telescope located at the Mediterranean Sea, near Toulon (France). It consists of 12 lines with 75 optical modules each, covering a height of $350 \mathrm{~m}$ and anchored at the sea floor at a depth of about $2.5 \mathrm{~km}$, with a separation of around $70 \mathrm{~m}$ between neighboring modules. The neutrino detection is based on the Cherenkov light emitted when the charged leptons produced by the neutrino interactions move through the water. Although ANTARES was not designed to contribute to the determination of the oscillation parameters, it was the first large volume Cherenkov-based neutrino telescope performing such analysis with atmospheric neutrinos. It managed to do it as a result of an important reduction of their threshold neutrino energy, from $50 \mathrm{GeV}$, when only multi-line events are considered, to $20 \mathrm{GeV}$ for single-line events.

We analyze atmospheric data from the ANTARES collaboration following Ref. [94], taking also into account matter effects, and including electron neutrino and neutral current interaction events. In Fig. 2.5 we plot the allowed regions in 

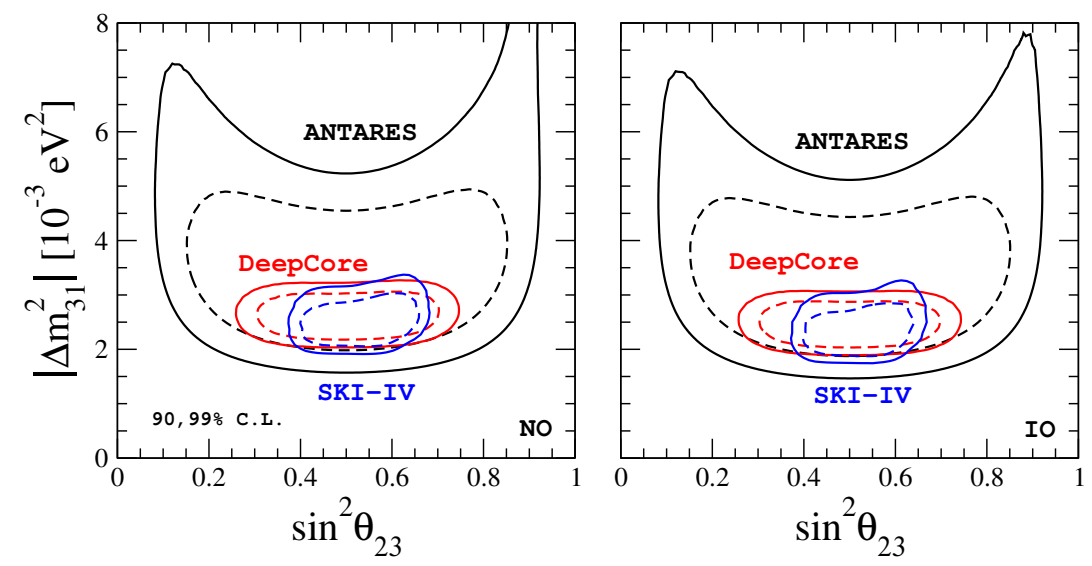

Figure 2.5: 90 and 99\% C.L. (2 d.o.f.) allowed regions at the $\sin ^{2} \theta_{23^{-}} \Delta m_{31}^{2}$ plane obtained from the atmospheric neutrino experiments for normal (left) and inverted ordering (right). Figure taken from Ref. [2].

the atmospheric parameters at 90 and $99 \%$ C.L. from our analysis of ANTARES data. One sees the regions are still very large and, therefore, the sensitivity is not competitive with the other experiments.

\section{Atmospheric neutrinos at Super-Kamiokande}

We include the most recent atmospheric neutrino results from the SuperKamiokande experiment [95], corresponding to the combined analysis of phases I to IV of the experiment, with a total of 328 kton-year exposure of the detector. The data analysis performed by the Super-Kamiokande collaboration, optimized to enhance the sensitivity to the neutrino mass ordering, includes the impact of the atmospheric oscillation parameters as well as the reactor angle and the $\mathrm{CP}$ phase. Since the experimental collaboration does not provide enough information, a reproduction of the results of this analysis is not possible outside of the collaboration. Because of that, we do not analyze Super-Kamiokande data ourselves, but only include the latest $\chi^{2}$-table made available by the collaboration 101. In Fig. 2.5 we show the regions in the atmospheric plane obtained by Super-Kamiokande. In later sections we will also show the results in the $\sin ^{2} \theta_{13}-\delta$ plane. One sees that Super-Kamiokande is the most sensitive experiment among the atmospheric experiments. 


\subsubsection{Accelerator experiments}

Long baseline accelerator neutrino experiments observe neutrinos which are created in accelerators from mesons decays. The mesons (typically pions and kaons) are created in the accelerator and then focused into a beam. Next they decay into muon-neutrinos, while a beam dump absorbs the ones which do not decay. Using different polarities of the focusing horn it is possible to separate mesons from antimesons, resulting in a mostly pure beam of neutrinos or antineutrinos. Note, however, that creating a really pure beam is not possible, hence, there will be always a background contamination of so called wrong-sign neutrinos. The long baseline accelerator experiments always consist of two detectors, one near detector measuring the initial neutrino flux, close to the accelerator complex, and a far detector measuring the oscillated neutrino flux. Long baseline accelerator experiments measure the appearance of $\nu_{e}$ from the initial $\nu_{\mu}$ flux, and also the disappearance of $\nu_{\mu}$. This makes them able to measure $\Delta m_{31}^{2}, \theta_{23}, \theta_{13}, \delta$ and, in principle, also the neutrino mass ordering. For our global fit we use data from several long baseline accelerator experiments: $\mathrm{NO} \nu \mathrm{A}$ [102], T2K 103 105, MINOS [106 and K2K [107.

\section{$\mathrm{NO} \nu \mathrm{A}$}

The $\mathrm{NO} \nu \mathrm{A}$ experiment is a long baseline neutrino oscillation facility with a $810 \mathrm{~km}$ baseline making it the largest long baseline experiment to date. It was designed to observe $\nu_{\mu}$-disappearance as well as $\nu_{e}$-appearance in both neutrino and antineutrino channels. In order to accomplish this, it uses an intense and (nearly) pure beam of $\nu_{\mu}$ generated at the Fermilab accelerator complex. These neutrinos go through the Earth to northern Minnesota to be detected at the Ash River far detector. Because of its $810 \mathrm{~km}$ baseline, it is more sensitive to matter effects than the T2K experiment. The detectors are $14 \mathrm{mrad}$ off-axis, which results in a narrow neutrino energy spectrum, peaked around $2 \mathrm{GeV}$, which coincides with the oscillation maximum for $\nu_{\mu} \rightarrow \nu_{e}$ oscillations.

The $\mathrm{NO} \nu \mathrm{A}$ experiment has collected an equivalent of $8.85 \times 10^{20}$ protons on target (POT) of data in neutrino mode and is now taking data with the antineutrino beam. In our global fit, we include the results for $\nu_{\mu}$-disappearance and $\nu_{e}$-appearance of the $\mathrm{NO} \nu \mathrm{A}$ experiment 102. In the disappearance channel, a total of 126 events have been observed, while 763 events were expected under the no-oscillation hypothesis. In the appearance channel, a total of 66 events have been detected. The neutrino oscillation analysis reported by the $\mathrm{NO} \nu \mathrm{A}$ collaboration imposing a prior on $\theta_{13}$ slightly disfavors inverted mass ordering, 
with a significance of approximately $2 \sigma$. Our simulation of the $\mathrm{NO} \nu \mathrm{A}$ experiment has been performed using GLoBES [108, 109], including all the systematic errors reported in 110,111] and updated in Refs. [102,112.

In Fig. 2.6 we compare the restrictions on the atmospheric neutrino parameters derived from long baseline accelerator data coming from the $\mathrm{T} 2 \mathrm{~K}, \mathrm{NO} \nu \mathrm{A}$ and MINOS experiments, at 90 and $99 \%$ confidence level. Further results are summarized in Figs. 2.7, 2.8, 2.9, 2.10 and 2.11 and discussed in the following section.

\section{T2K}

Apart from solar and atmospheric neutrinos, Super-K receives also neutrinos from the $295 \mathrm{~km}$ distant J-PARC accelerator complex in Tokai. This experiment is referred to as T2K (Tokai-to-Kamioka). This analysis includes data from T2K in both neutrino and antineutrino mode, as published in Refs. 103 105. With an accumulated statistics of $14.6 \times 10^{20}$ POT in the neutrino run, the T2K collaboration now observes 240 disappearance and $74+15$ appearance (charged current quasi-elastic and charged current single-pion, respectively) neutrino events. Note, however, that the CC- $1 \pi$ appearance events have not been included in our simulation, since at this stage their contribution to the total sensitivity is very small. In the antineutrino channel, with $7.6 \times 10^{20} \mathrm{POT}$, a total of 68 disappearance $\bar{\nu}_{\mu}$ events and 7 appearance $\bar{\nu}_{e}$ events were recorded. In the present analysis we have included the newest neutrino fluxes at Super-K provided by the T2K collaboration [113]. The simulation of the experiment and the statistical analysis were performed with the GLoBES package [108, 109], including all systematic uncertainties reported in Ref. [105].

The results of our analysis of $\mathrm{T} 2 \mathrm{~K}$ data projected into the atmospheric plane are shown in Fig. 2.6. T2K has achieved already some CP sensitivity, as seen in Fig. 2.7. that will be discussed in the next section. Indeed, thanks to the combination of the results in the neutrino and the antineutrino channel, T2K is the first experiment able to exclude on its own certain values of the $\mathrm{CP}$ phase at more than $2 \sigma$ for normal ordering (NO), and even at $3 \sigma$ for inverted ordering (IO). The allowed regions for other oscillation parameters, such as $\theta_{13}$ and $\Delta m_{31}^{2}$, are found to be consistent with the measurements of reactor experiments.

\section{MINOS}

The Main Injector Neutrino Oscillation Search (MINOS) experiment was the precursor of $\mathrm{NO} \nu \mathrm{A}$. It consisted of a 1 kton near detector in Fermilab and a 


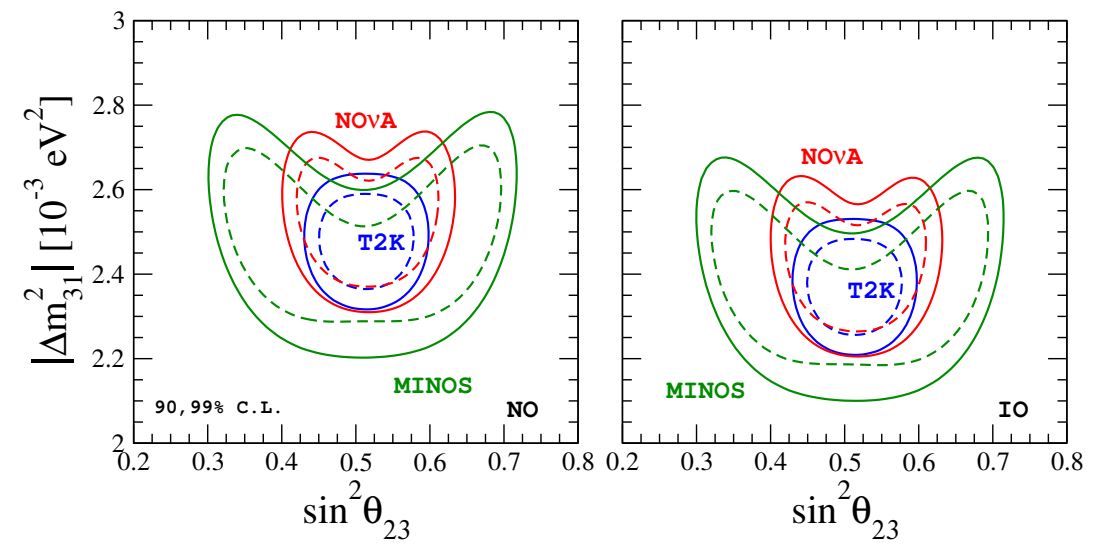

Figure 2.6: 90 and $99 \%$ C.L. (2 d.o.f.) allowed regions at the $\sin ^{2} \theta_{23^{-}} \Delta m_{31}^{2}$ plane for normal (left) and inverted mass ordering (right) as restricted from the long baseline accelerator experiments. Figure taken from Ref. [2].

5.4 kton far detector at the Soudan mine, $735 \mathrm{~km}$ away. A special feature of MINOS was the use of plastic and also magnetized steel-scintillator as detection material. Making use of the magnetic field it was possible to distinguish $\mu^{-}$from $\mu^{+}$, hence distinguishing the incoming neutrinos from antineutrinos.

In our global fit, we use the latest MINOS data release [106], where we consider only the beam data. The data has been collected over nine years and correspond to an exposure of $10.71 \times 10^{20} \mathrm{POT}$ in the neutrino mode and $3.36 \times 10^{20} \mathrm{POT}$ in the antineutrino mode. One of the key features of this data sample is the preference for a non-maximal value of the atmospheric mixing angle $\theta_{23}$. From the official MINOS analysis, one obtains that maximal mixing is disfavored at $86 \%$ C.L. The result of our analysis of MINOS data is shown in Fig. 2.6. One can see that, in the $\theta_{23}$-range preferred by $\mathrm{T} 2 \mathrm{~K}$ and $\mathrm{NO} \nu \mathrm{A}$, the MINOS measurement of $\Delta m_{31}^{2}$ is still comparable to the one of the newer experiments.

\section{$2.2 \quad$ Global fit results}

We now describe the results of the combined neutrino oscillation fit. The solar parameters are measured by the solar experiments and KamLAND, as discussed in Sec. 2.1.1. There is no contribution from the other experiments to these parameters. As can be seen in Fig. 2.3, the solar mixing angle is best determined by the solar experiments, while the best measurement of the solar mass splitting comes from KamLAND. Note that KamLAND on its own would also allow a re- 
gion for $\sin ^{2} \theta_{12}>0.5$, but this region is excluded by solar data. In the analysis of atmospheric data, we use data from the ANTARES and IceCube collaborations as well as from Super-Kamiokande. As seen in Fig. 2.5, the 863-day atmospheric data from ANTARES and the 3-year data from IceCube DeepCore are enough to provide a determination of the atmospheric oscillation parameters. Note, however, that the determination of $\theta_{23}$ from atmospheric data is still dominated by the analysis of Super-Kamiokande. In any case, the neutrino telescope results are in complete consistency with what follows from the Super-Kamiokande atmospheric data, leading to a clear global picture for the all-atmospheric data fit, shown in Fig. 2.5 .

Concerning the long baseline accelerator data, Fig. 2.6 shows the regions allowed by the $\mathrm{NO} \nu \mathrm{A}$ and $\mathrm{T} 2 \mathrm{~K}$ neutrino results, as well as the older MINOS data sample. In comparison with Fig. 2.5, one sees that atmospheric parameters are mainly constrained by long baseline accelerator data, and that all the results are in agreement with maximal atmospheric mixing. On the other hand, Fig. 2.4 shows how the new reactor data, clearly dominated by Daya Bay, provide a significantly improved determination of $\theta_{13}$. It also illustrates the important role of reactor neutrino data in mapping out the allowed region of the atmospheric squared mass splitting parameter.

In what follows, we highlight the main features of our neutrino oscillation global fit results, focusing upon the main open challenges of the three-neutrino picture: $\mathrm{CP}$ violation, the neutrino mass ordering and the $\theta_{23}$ octant problem.

\section{Sensitivity to CP violation}

Long baseline accelerator neutrino oscillation data play an important role in determining the $\mathrm{CP}$ violating phase, $\delta$. In order to highlight this point we present the $\Delta \chi^{2}$-profile for the CP phase, as determined from T2K, NO $\nu \mathrm{A}$ and Super-K atmospheric data alone, as well as by the global oscillation data sample, as shown in the right panels in Fig. 2.7. Note that, here, the $\Delta \chi^{2}$-profile has been obtained from the local minimum for each mass ordering.

This result shows how the current global sensitivity to the CP phase is dominated by the $\mathrm{T} 2 \mathrm{~K}$ experiment, with added rejection against $\delta=\pi / 2$ obtained after combining with the other experiments. Indeed, we find that the combination with reactor data is crucial to enhance the rejection against $\delta=\pi / 2$. As a result, we find that, in the global analysis, $\delta=\pi / 2$ is disfavored with $\Delta \chi^{2}=22.9$ $(4.8 \sigma)$ for normal ordering. The rejection against $\delta=\pi / 2$ is found to be stronger for inverted mass spectrum, where it is excluded with $\Delta \chi^{2}=37.3(6.1 \sigma)$, with respect to the minimum for this ordering. As can also be seen from the figure, the 

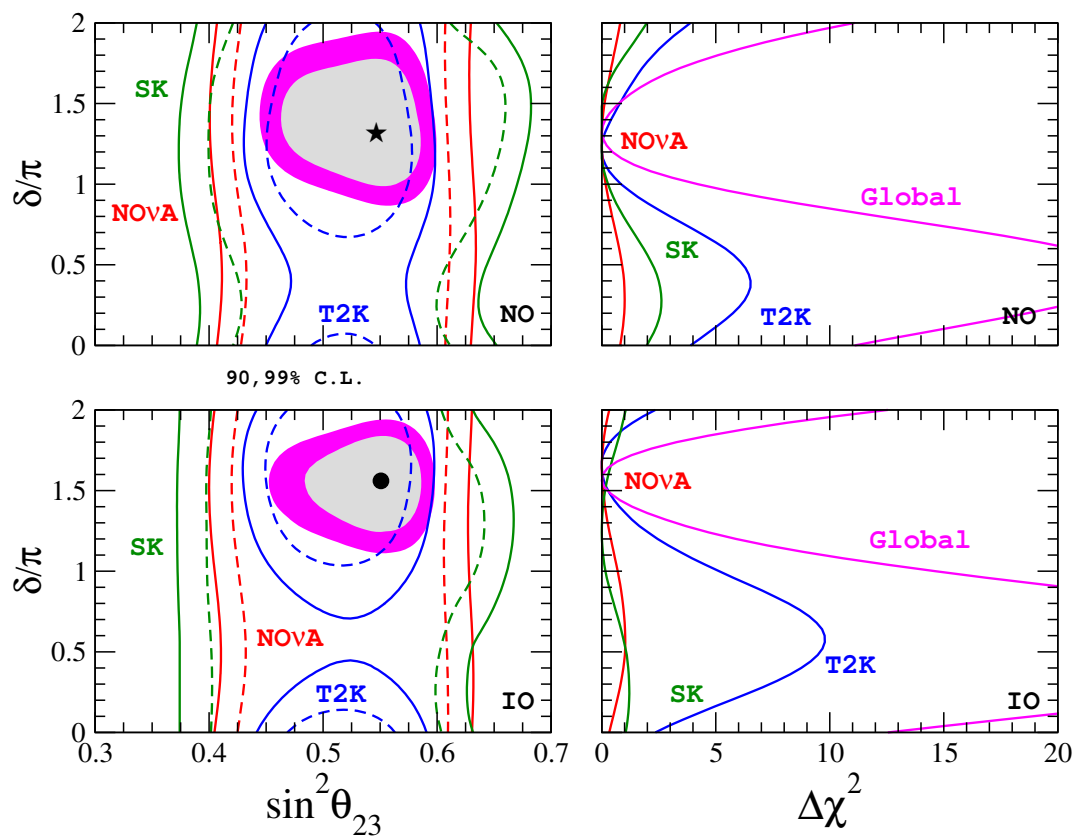

Figure 2.7: Left: 90 and 99\% C.L. (2 d.o.f.) regions from T2K (blue lines) and $\mathrm{NO} \nu \mathrm{A}$ (red) data, from the atmospheric Super-K results (green) and from the global fit of all the oscillation experiments (colored regions). The star indicates the best fit point from our global analysis, found for normal mass ordering, while the black dot indicates the local minimum for inverted mass ordering. Right: $\Delta \chi^{2}$-profile as a function of the $\mathrm{CP}$ phase $\delta$ from $\mathrm{T} 2 \mathrm{~K}, \mathrm{NO} \nu \mathrm{A}$ and Super-K atmospheric (with the same color code as in the left panel) and from the global fit (magenta). In both cases, the upper (lower) panels correspond to normal (inverted) mass ordering. Figure taken from Ref. [2].

current preferred value of $\delta$ depends on the mass ordering, lying closer to $3 \pi / 2$ for inverted ordering. The current best fit values for the $\mathrm{CP}$ violating phase are located at $\delta=1.32 \pi$ for NO and at $\delta=1.56 \pi$ for IO. Due to its cyclic nature, the likelihood of the CP phase does not necessarily behave like a $\chi^{2}$ distribution [114. Our result, however, is still valid. In Ref. [4] we performed a Bayesian analysis, obtaining the same result as in our frequentist treatment here. There, we rearranged the boundaries of the $\mathrm{CP}$ phase such that the best fit value corresponds to the center of the interval. 


\section{Neutrino mass ordering}

Concerning the sensitivity to the neutrino mass ordering, our global fit shows for the first time a hint in favor of normal neutrino mass ordering, with inverted ordering disfavored with $\Delta \chi^{2}=11.7(3.4 \sigma)$. Note that there has been some confusion in the literature when trying to quantify the preference of one mass ordering over the other 115 119. Here, we can safely assume that our result is robust, since we obtain a very similar preference in the Bayesian analysis performed in Ref. [4] with the same data set. In order to disentangle the origin of the preference for NO in our global analysis, we display in Figs. 2.8 and 2.9 the allowed regions for $\theta_{23}, \theta_{13}$ and $\delta$ for NO and IO for different data set combinations: long baseline accelerator data only, long baseline accelerator plus atmospheric, long baseline accelerator plus reactors and the combination of all data sets. Down-triangles indicate the best fit points obtained in the analysis of long baseline accelerator data, squares correspond to the best fit points derived from the combination of long baseline accelerator plus atmospheric, while up-triangles are the best fit point for long baseline accelerator plus reactor data. The star and black dot follow the same convention as in Fig. 2.7.

The black lines in these figures delimit the allowed regions from the combination of all long baseline accelerator data discussed above. In principle, given the small impact of matter effects in the neutrino propagation at such baselines, one would expect a limited sensitivity of the current long baseline accelerator experiments to the neutrino mass ordering. Indeed, this is confirmed by our independent analysis of $\mathrm{T} 2 \mathrm{~K}$ and $\mathrm{NO} \nu \mathrm{A}$ data, that shows only a slight preference for normal mass ordering at the level of $\Delta \chi^{2} \sim 1$. However, the combined analysis of long baseline accelerator and reactor data (see cyan lines in the figures) results in an enhanced sensitivity to the mass ordering which, in all cases, favors normal over inverted mass ordering. This happens due to the mismatch between the values of $\theta_{13}$ preferred by reactor and long baseline accelerator experiments, which is larger for the inverted mass ordering, as shown in Figs. 2.8 and 2.9. While in normal ordering the best fit for long baseline accelerator experiments alone, $\sin ^{2} \theta_{13}=0.026$, is relatively close to the global one, $\sin ^{2} \theta_{13} \simeq 0.022$, mainly constrained by reactors, this is not the case for inverted ordering, where long baseline accelerator data prefer $\sin ^{2} \theta_{13}=0.031$. As a result, the combined analysis of reactor and long baseline accelerator data shows better agreement under the normal mass ordering hypothesis. For instance, a combined analysis of the latest $\mathrm{NO} \nu \mathrm{A}$ results with reactor data indicates a preference for normal ordering with $\Delta \chi^{2}=3.7$. In the case of $\mathrm{T} 2 \mathrm{~K}$, the combination with reactor data results in a stronger preference for normal over inverted mass ordering, with $\Delta \chi^{2}=5.3$. 

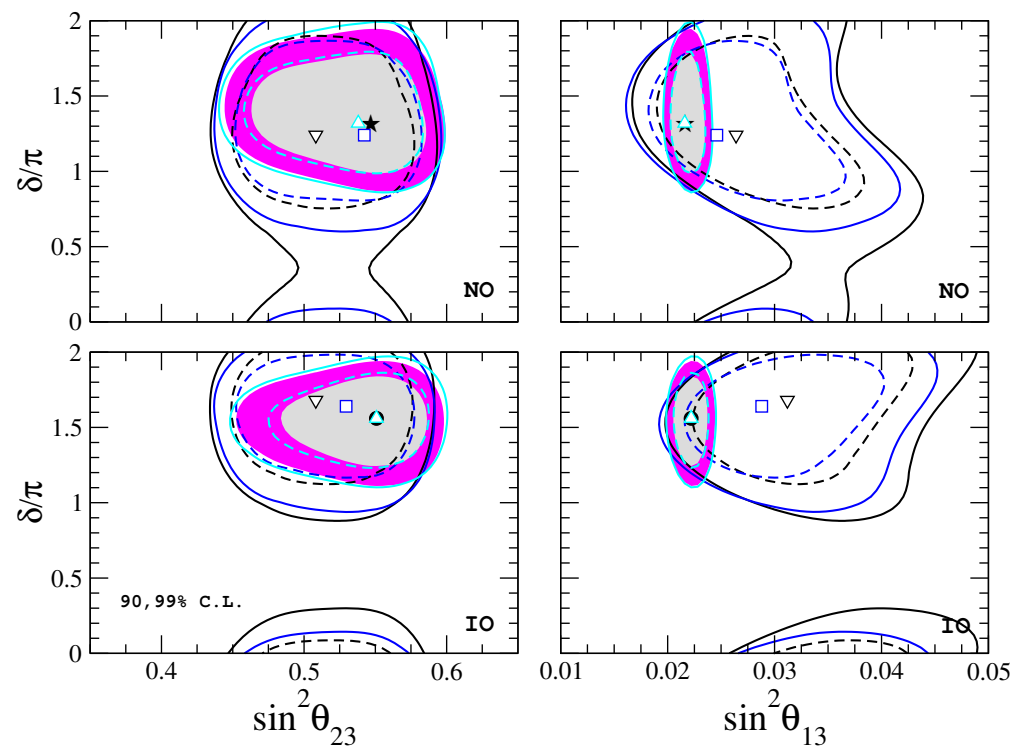

Figure 2.8: 90 and $99 \%$ C.L. (2 d.o.f.) allowed regions in the $\sin ^{2} \theta_{23}-\delta$ (left) and $\sin ^{2} \theta_{13}-\delta$ (right) planes from long baseline accelerator data only (black lines), long baseline accelerator plus atmospheric (blue), long baseline accelerator plus reactors (cyan) and from the global fit of all experiments (colored regions). Upper (lower) panels correspond to normal (inverted) mass ordering. The best fit points are indicated by black down-triangles (long baseline accelerator data), blue squares (long baseline accelerator plus atmospheric), cyan up-triangles (long baseline accelerator plus reactors), as well as stars and black dots, following the same convention as in Fig. 2.7. Figure taken from Ref. [2].

This enhanced sensitivity to the mass ordering is due to the tension that exists between the value of the atmospheric mass splitting preferred by reactor, mainly Daya Bay, and T2K. One finds that Daya Bay prefers a higher value for $\Delta m_{31}^{2}$ with respect to the one indicated by $\mathrm{T} 2 \mathrm{~K}$, and the difference is larger for inverted mass ordering. The combined analysis of all long baseline accelerator and reactor data yields a preference for normal mass ordering with $\Delta \chi^{2}=7.5$.

By combining these data samples with atmospheric data, one gets the final global results indicated by the colored regions in Figs. 2.8 and 2.9. In principle, one may expect the largest sensitivity to the neutrino mass ordering to come from the observation of matter effects in the atmospheric neutrino flux. However, we find that the neutrino telescope experiments IceCube DeepCore and ANTARES are not yet very sensitive to the mass ordering. In fact, the difference between 
normal and inverted mass ordering from the combined analysis of DeepCore and ANTARES is only $\Delta \chi^{2}=0.4$, obtained mainly from IceCube DeepCore data. On the other hand, the most recent analysis of atmospheric data sample of the Super-K experiment shows an enhanced sensitivity to the mass ordering compared to previous ones. Indeed, Super-K data alone disfavors the inverted mass ordering with $\Delta \chi^{2}=3.5$. If a prior on the reactor mixing angle is imposed in the atmospheric data analysis, the sensitivity rises up to $\Delta \chi^{2}=4.3$ [95]. The effect of adding the atmospheric data to the global analysis is not very noticeable in Figs. 2.8 and 2.9 , where the allowed regions with and without atmospheric data are similar. However, the impact of atmospheric data in the global sensitivity to the mass ordering allows one to disfavor inverted mass ordering at $\Delta \chi^{2}=11.7$. This result is very relevant, since from the combination of the different types of neutrino experiments we can obtain for the first time a preference for normal neutrino mass ordering slightly above $3 \sigma$.

\section{The $\theta_{23}$ octant problem}

The role of long baseline accelerator, atmospheric and reactor experiments in selecting the $\theta_{23}$-octant is illustrated in Figs. 2.8 and 2.9. They stress the complementary role of the different oscillation data samples on the possible discrimination of the $\theta_{23}$ octant. In fact, as noticed in Ref. [120] and recently in Ref. [121], an improved measurement of the reactor angle helps resolving the atmospheric octant. From the figures, we see that the analysis of long baseline accelerator data only (indicated by black lines) shows a preference for values of $\theta_{23}$ close to maximal mixing for the two mass orderings, with the best fit points indicated by a down-triangle. For both mass orderings we find a preferred value of $\sin ^{2} \theta_{23}=0.508$. The combination with the atmospheric data sets (illustrated by the blue lines in the figures) provides a further constraint on the allowed region for $\theta_{23}$. Moreover, the inclusion of atmospheric data in the analysis produces a shift of the best fit value of $\theta_{23}$ to larger values for both mass orderings $\left(\sin ^{2} \theta_{23}=0.54\right.$ for $\mathrm{NO}$ and $\sin ^{2} \theta_{23}=0.53$ for IO), although values of $\theta_{23}$ in the first octant are still allowed with $\Delta \chi^{2} \geq 0.8$ (2.0) for NO (IO). The combination with reactor experiments in the global neutrino fit (colored regions in the figures) moves the best fit value of $\theta_{23}$ to larger values for both mass orderings, leading to $\sin ^{2} \theta_{23} \simeq 0.55$ as the preferred value. Moreover, reactor data also modify the preferred values of $\theta_{13}$ and $\delta$, from the values fixed by the combination of long baseline accelerator and atmospheric data, as shown in Figs. 2.8 and 2.9. For both mass orderings $\delta$ is pushed towards $3 \pi / 2$, while the reactor mixing angle is slightly shifted towards smaller values. One should stress that reactor data, 

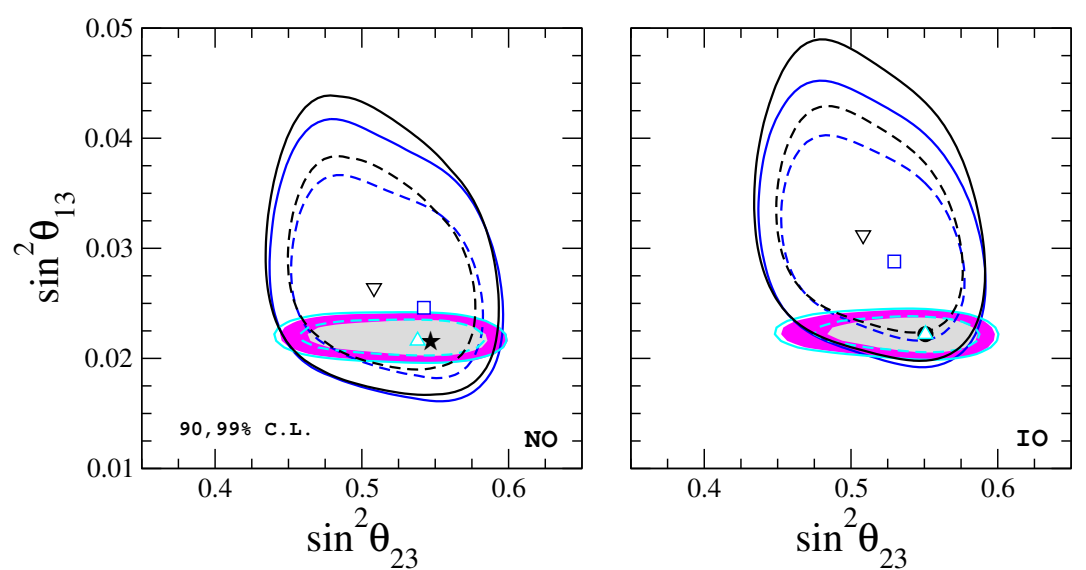

Figure 2.9: 90 and 99\% C.L. (2 d.o.f.) regions from the combination of different neutrino data samples. The convention used to indicate the regions and best fit points is the same as in Fig. 2.8. Figure taken from Ref. [2].

specially Daya Bay and RENO, are crucial to the determination of the allowed region for $\theta_{13}$. Going back to the octant preference, we would like to remark that the indications described above are still far from robust. Indeed, values of the atmospheric mixing angle below $45^{\circ}$ are allowed at $\Delta \chi^{2} \geq 1.6$ for the case of normal ordering and at $\Delta \chi^{2} \geq 3.2$ for inverted ordering with respect to the minimum in this mass spectrum.

Despite the recent progress on this matter, the octant discrimination problem lies far beyond the current generation of neutrino oscillation experiments, and will be a particularly stubborn problem in the years to come. On the positive side, however, it has been noted that the task of octant discrimination and probing for leptonic $\mathrm{CP}$ violation in current and future long baseline accelerator experiments can be facilitated by prior model-specific theoretical knowledge of the predicted pattern of leptonic mixing. See, as an example, Figure 1 given in 122 and the associated discussion.

\subsection{Summary and discussion}

We have discussed in detail the status of the mass ordering, CP violation and octant discrimination, analyzing the interplay among the different neutrino oscillation data samples. The results obtained in our global fit are summarized 


\begin{tabular}{|lccc|}
\hline parameter & best fit $\pm 1 \sigma$ & $2 \sigma$ range & $3 \sigma$ range \\
\hline$\Delta m_{21}^{2}\left[10^{-5} \mathrm{eV}^{2}\right]$ & $7.55_{-0.16}^{+0.20}$ & $7.20-7.94$ & $7.05-8.14$ \\
$\left|\Delta m_{31}^{2}\right|\left[10^{-3} \mathrm{eV}^{2}\right](\mathrm{NO})$ & $2.50 \pm 0.03$ & $2.44-2.57$ & $2.41-2.60$ \\
$\left|\Delta m_{31}^{2}\right|\left[10^{-3} \mathrm{eV}^{2}\right](\mathrm{IO})$ & $2.42_{-0.04}^{+0.03}$ & $2.34-2.47$ & $2.31-2.51$ \\
$\sin ^{2} \theta_{12} / 10^{-1}$ & $3.20_{-0.16}^{+0.20}$ & $2.89-3.59$ & $2.73-3.79$ \\
$\theta_{12} /^{\circ}$ & $34.5_{-1.0}^{+1.2}$ & $32.5-36.8$ & $31.5-38.0$ \\
$\sin ^{2} \theta_{23} / 10^{-1}(\mathrm{NO})$ & $5.47_{-0.30}^{+0.20}$ & $4.67-5.83$ & $4.45-5.99$ \\
$\theta_{23} /^{\circ}$ & $47.7_{-1.7}^{+1.2}$ & $43.1-49.8$ & $41.8-50.7$ \\
$\sin ^{2} \theta_{23} / 10^{-1}(\mathrm{IO})$ & $5.51_{-0.30}^{+0.18}$ & $4.91-5.84$ & $4.53-5.98$ \\
$\theta_{23} /^{\circ}$ & $47.9_{-1.7}^{+1.0}$ & $44.5-48.9$ & $42.3-50.7$ \\
$\sin ^{2} \theta_{13} / 10^{-2}(\mathrm{NO})$ & $2.160_{-0.069}^{+0.083}$ & $2.03-2.34$ & $1.96-2.41$ \\
$\theta_{13} /{ }^{\circ}$ & $8.45_{-0.14}^{+0.16}$ & $8.2-8.8$ & $8.0-8.9$ \\
$\sin ^{2} \theta_{13} / 10^{-2}(\mathrm{IO})$ & $2.220_{-0.076}^{+0.074}$ & $2.07-2.36$ & $1.99-2.44$ \\
$\theta_{13} /^{\circ}$ & $8.53_{-0.15}^{+0.14}$ & $8.3-8.8$ & $8.1-9.0$ \\
$\delta / \pi(\mathrm{NO})$ & $1.32_{-0.15}^{+0.21}$ & $1.01-1.75$ & $0.87-1.94$ \\
$\delta /{ }^{\circ}$ & $238_{-27}^{+38}$ & $182-315$ & $157-349$ \\
$\delta / \pi(\mathrm{IO})$ & $1.56_{-0.15}^{+0.13}$ & $1.27-1.82$ & $1.12-1.94$ \\
$\delta /{ }^{\circ}$ & $281_{-27}^{+23}$ & $229-328$ & $202-349$ \\
\hline
\end{tabular}

Table 2.2: Summary of neutrino oscillation parameters as determined from this global analysis. The ranges for inverted ordering refer to the local minimum for this neutrino mass ordering.

in Table 2.2 as well as Figs. 2.10 and 2.11 for normal and inverted mass ordering. Some comments are in order.

First, we note that the improved precision on $\theta_{13}$ follows mainly from the Daya Bay and RENO data. Thanks to the combination of T2K neutrino and antineutrino data, we have now an improved sensitivity to CP violation. Indeed, $\mathrm{T} 2 \mathrm{~K}$ is the first experiment showing a sensitivity on its own, excluding some values of $\delta$ before combining with reactor data. In this analysis, we have obtained a strong preference for values of the $\mathrm{CP}$ phase in the range $[\pi, 2 \pi]$, excluding values close to $\pi / 2$ at more than $4 \sigma$. Concerning the octant of $\theta_{23}$, this global analysis prefers the second octant slightly. We have found that, for normal neutrino mass ordering, the upper atmospheric octant is now preferred with $\Delta \chi^{2}=1.6$, while for the case of inverted ordering, values of the atmospheric mixing angle in the 
lower octant are allowed with $\Delta \chi^{2} \geq 3.2$. More remarkably, our global analysis favors for the first time the normal mass ordering over the inverted one at $3.4 \sigma$. As discussed in the previous section, part of the sensitivity to the mass ordering comes from the most recent atmospheric analysis of Super-K. This new analysis shows a preference for normal over inverse mass ordering with $\Delta \chi^{2}=3.5$. On the other hand, a mismatch between the values of $\theta_{13}$ preferred by long baseline accelerator and reactor data (larger for IO) also gives a relevant contribution to the global sensitivity to the mass ordering. This effect is also enhanced due to a tension between the preferred values of the atmospheric mass splitting by $\mathrm{T} 2 \mathrm{~K}$ and reactor experiments.
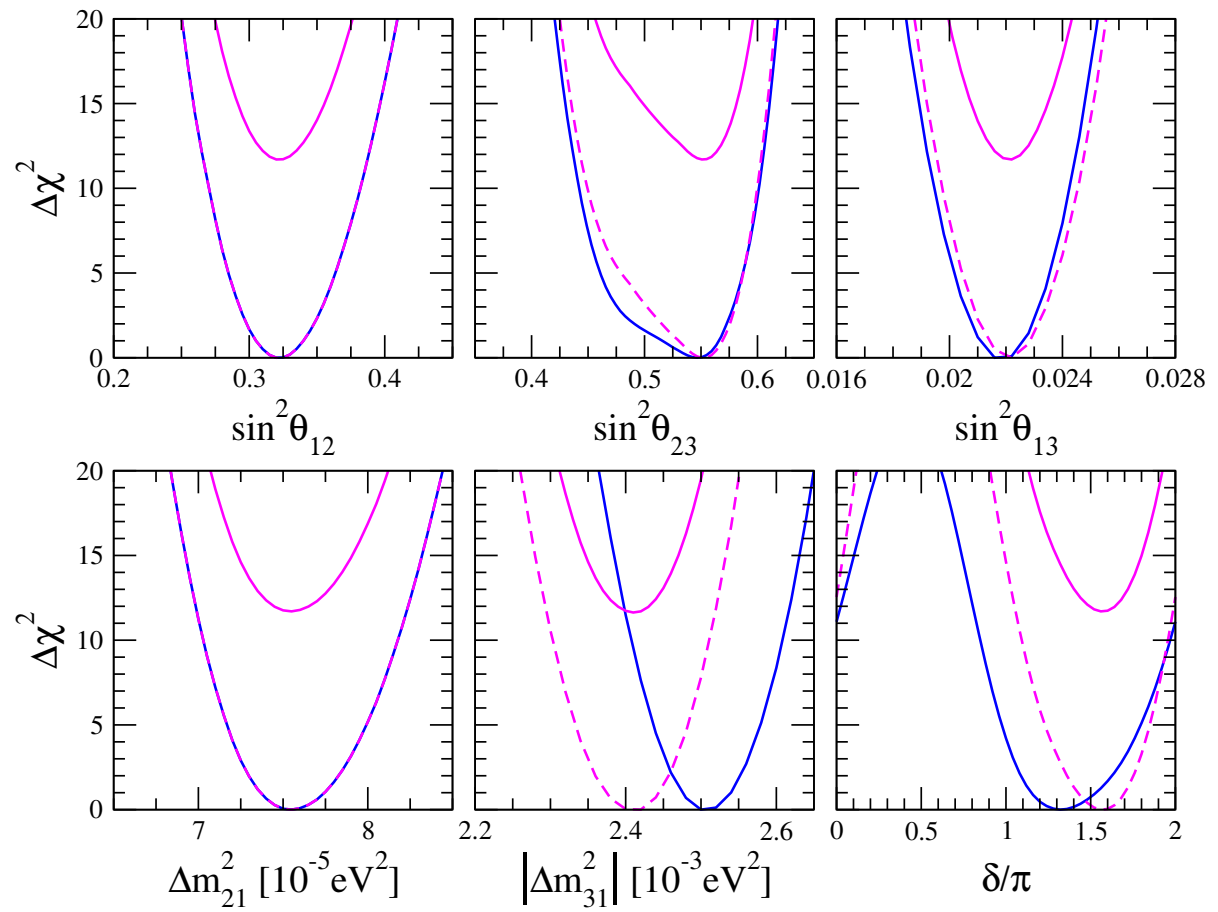

Figure 2.10: Summary of neutrino oscillation parameters, 2018. Blue lines correspond to NO and magenta lines to IO. The $\Delta \chi^{2}$-profiles for inverted ordering are plotted with respect to the minimum for this neutrino mass ordering (dashed) as well as with respect to the global minimum (solid lines). Figure taken from Ref. [2].

In short, we have seen how the precision in the determination of the bestknown oscillation parameters has improved thanks to the recent accelerator and 
reactor neutrino oscillation data. Also the sensitivity to mass ordering, CP violation and the octant of the atmospheric angle has improved, although we are still quite far from a robust measurement, especially of the octant. Note, however, that the presence of new physics beyond the Standard Model may affect significantly the results obtained within the current neutrino oscillation picture. For example, nonstandard neutrino interactions with matter or non-unitary neutrino mixing, expected in seesaw models of neutrino mass generation, may significantly reduce the precision in the determination of neutrino oscillation parameters due to the presence of correlations and degeneracies with new physics parameters. Conversely, however, such well-motivated beyond-standard scenarios can also bring in new opportunities for current and future long baseline accelerator neutrino oscillation experiments.

As a final remark, let us mention that the results from our global fit are in good agreement with the ones performed by $\nu$-fit 123 and the Bari-group [124]. Minor differences are due to slightly different data sets included in the analyses. 

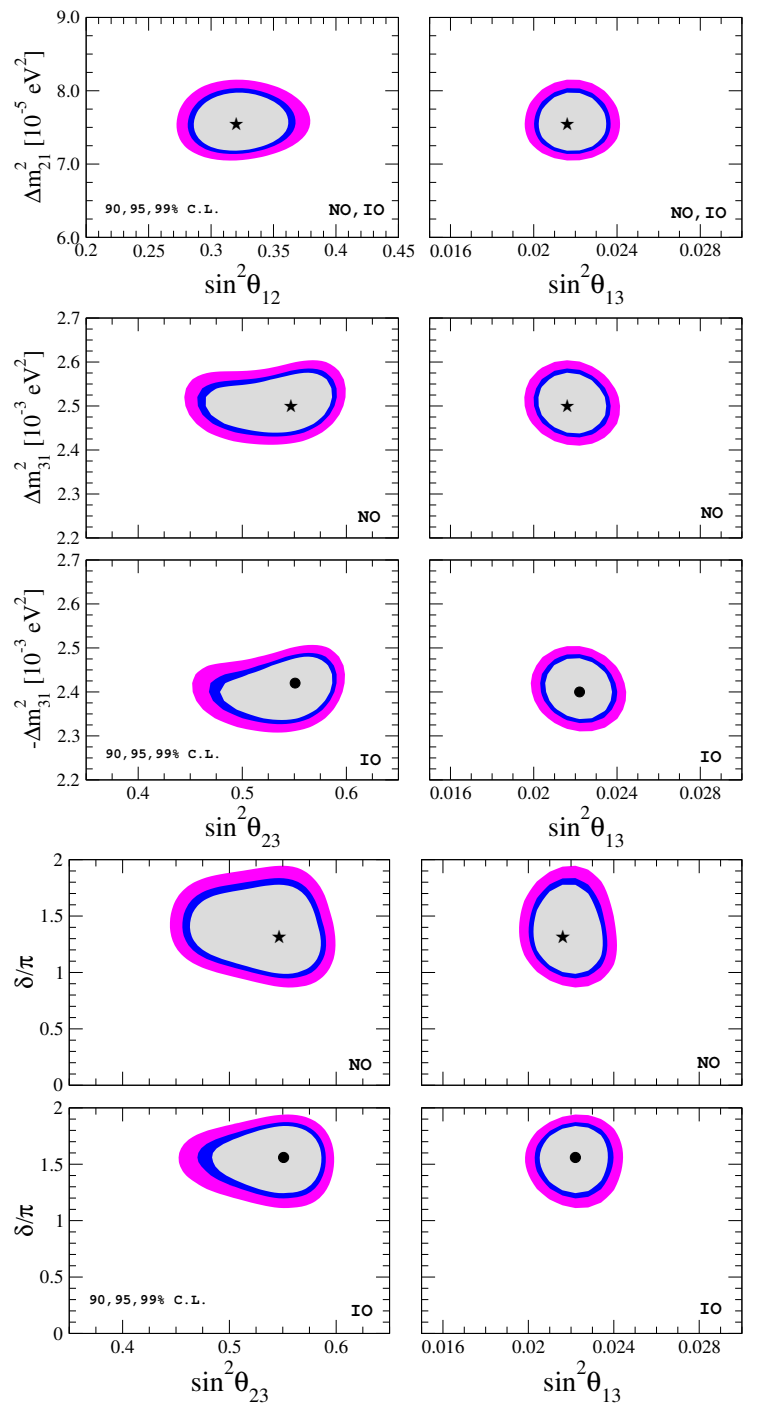

Figure 2.11: Global fit summary 2018. In the two four-panel figures, the upper ones correspond to normal ordering and the lower ones to inverted mass ordering. Global fit regions correspond to 90, 95 and 99\% C.L. (2 d.o.f.). Regions for inverted ordering are plotted with respect to the minimum for this neutrino mass ordering. Figure taken from Ref. [2]. 



\section{Chapter 3}

\section{Global fit to oscillation data without CPT invariance}

This chapter covers our first departure from the standard model. In the last two sections, we first explained the physics behind neutrino oscillations and, next, we obtained the current best fit values and allowed regions of the parameters describing these oscillations. In scenarios containing physics beyond the Standard Model, this description might be incomplete, however. For example, in theories that violate the CPT symmetry, neutrinos and antineutrinos could have, in principle, different masses and also different mixing parameters, resulting in a different oscillation behavior for neutrinos and antineutrinos. Here, we calculate the current bounds on CPT violation in the neutrino sector by assuming different oscillation parameters for neutrinos and antineutrinos which, by CPT theorem, should be equal. We will not assume any specific model of CPT violation but rather compute general (model independent) bounds. The results of this chapter are published in Ref. [5].

\subsection{Introduction}

CPT invariance is arguably one of the few sacred cows of particle physics. Its position as such arises from the fact that CPT conservation is a natural consequence of only three assumptions: Lorentz invariance, locality and hermiticity of the Hamiltonian, all of which have plenty of reasons to be included in our theories, besides CPT itself. In short, the CPT theorem states that particle and antiparticle have the same mass and, if unstable, also the same lifetime (for a nice proof of the CPT theorem see Ref. [125]). Therefore, the consequences of 
finding evidence of CPT non-conservation would be gigantic [126]. At least one of the three ingredients above must be false and our model building strategy would need to be revisited.

It should be noted, however, that testing the predictions of CPT conservation is not strictly equivalent to constraining $\mathrm{CPT}$ violation. Tests of CPT conservation might be performed by comparing the masses of particles and antiparticles. Indeed, these mass differences might be regarded as CPT violating observables. Nevertheless, the interpretation and comparison of bounds from different observables would only be possible with the consideration of a particular model of CPT violation.

Having said that, it is also clear that tests of CPT invariance have been historically associated with the neutral kaon system and, therefore, although in the absence of an explicit model any connection is meaningless, the comparison between kaons and neutrinos seems unavoidable. A superficial face value extrapolation leaves no room to be optimistic: the current limits on CPT violation arising from the neutral Kaon system seem to be quite solid

$$
\frac{\left|m\left(K^{0}\right)-m\left(\bar{K}^{0}\right)\right|}{m_{K}}<0.6 \times 10^{-18} .
$$

However, the strength of this limit is indeed artificial. Its robustness derives from the choice of the scale in the denominator, which is arbitrary at any rate and has nothing to do with a concrete model of CPT violation. Besides, the Kaon is not an elementary particle and, therefore, this test has more to do with testing QCD rather than a fundamental symmetry of (elementary) fermions. Additionally, the parameter present in the Lagrangian is not the mass but the mass squared and, therefore, this limit should be re-written as

$$
\left|m^{2}\left(K^{0}\right)-m^{2}\left(\bar{K}^{0}\right)\right|<0.25 \mathrm{eV}^{2} .
$$

Now it becomes obvious that neutrino experiments can test CPT to an unprecedented extent and, therefore, can provide stronger limits than the ones regarded as the most stringent now ${ }^{1}$ Let us stress again, however, that without an explicit model for CPT violation it is not straightforward or even meaningful to compare the neutrino-antineutrino mass squared differences and the kaon ones.

\footnotetext{
${ }^{1} \mathrm{CPT}$ was tested also using charged leptons. However, these measurements involve a combination of mass and charge and are not a direct CPT test. Only neutrinos can provide CPT tests on an elementary mass not contaminated by charge.
} 
CPT violation may show up only in one of the sectors and, therefore, the strong bounds in one of them might not be directly applicable to the other.

On the other hand, there are reasons to believe neutrinos are an ideal probe for CPT violation: quantum gravity is assumed to be non-local, opening the door to a potential CPT violation. Its effects, however, are expected to be Planck suppressed, i.e. $\langle v\rangle^{2} / M_{\mathrm{P}}$, exactly in the right ballpark for neutrino experiments to see them.

Furthermore, as it is well known, neutrinos offer a unique mass generation mechanism, the see-saw and, therefore, their masses are sensitive to new physics and new scales. Scales where non-locality could be expected to show up. Of course, in lack of a concrete theory of flavor, let alone one of CPT violation, the difference in the spectra of neutrinos and antineutrinos can appear not only in the mass eigenstates but also in the mixing between flavor and mass eigenstates. Neutrino oscillation experiments can test only CPT in the mass differences and mixing angles. An overall shift on the spectrum of neutrinos relative to that of antineutrinos cannot be detected in oscillation experiments and can be bound only by cosmological data, see Ref. [127]. It is important to notice that future kinematical direct searches for neutrino mass [128, 129 are not competitive with antineutrino mass searches [130] and thus cannot be used as a CPT test on the absolute mass scale either. For a schematic CPT-violating spectrum see Fig. 3.1.

Studies separating neutrinos and antineutrinos were done in the past 103 131133 under several assumptions. In Ref. 134 the authors obtained the following model-independent bounds on CPT invariance for the different parameters:

$$
\begin{aligned}
\left|\Delta m_{21}^{2}-\Delta \bar{m}_{21}^{2}\right| & <5.9 \times 10^{-5} \mathrm{eV}^{2}, \\
\left|\Delta m_{31}^{2}-\Delta \bar{m}_{31}^{2}\right| & <1.1 \times 10^{-3} \mathrm{eV}^{2}, \\
\left|\sin ^{2} \theta_{12}-\sin ^{2} \bar{\theta}_{12}\right| & <0.25, \\
\left|\sin ^{2} \theta_{13}-\sin ^{2} \bar{\theta}_{13}\right| & <0.03, \\
\left|\sin ^{2} \theta_{23}-\sin ^{2} \bar{\theta}_{23}\right| & <0.44,
\end{aligned}
$$

at $3 \sigma$. Note that we assume the neutrino oscillations being parameterized by the usual PMNS matrix $U_{\text {PMNS }}$, with parameters $\theta_{12}, \theta_{13}, \theta_{23}, \Delta m_{21}^{2}, \Delta m_{31}^{2}, \delta$, while the antineutrino oscillations are parameterized by a matrix $\bar{U}_{\text {PMNS }}$ with parameters $\bar{\theta}_{12}, \bar{\theta}_{13}, \bar{\theta}_{23}, \Delta \bar{m}_{21}^{2}, \Delta \bar{m}_{31}^{2}, \bar{\delta}$. This results in the same probability functions for antineutrinos as for neutrinos with the neutrino parameters replaced by their antineutrino counterparts, besides the standard change of sign in the $\mathrm{CP}$ phase. 

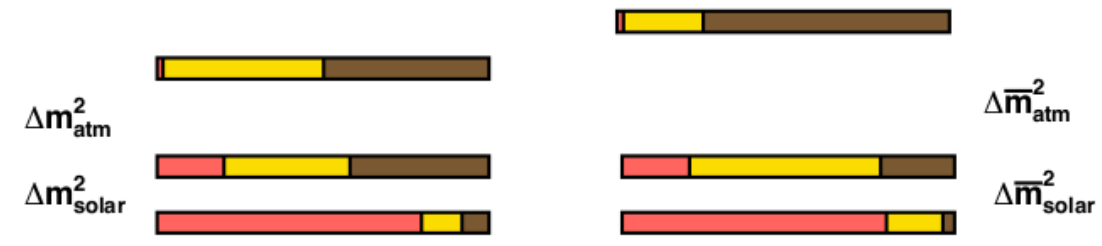

Figure 3.1: Generic CPT violating spectrum. We have not included an overall shift between the neutrino and antineutrino sector as it cannot be tested by oscillation experiments.

MINOS has also bounded the difference in the atmospheric mass-splitting to be

$$
\left|\Delta m_{31}^{2}-\Delta \bar{m}_{31}^{2}\right|<0.8 \times 10^{-3} \mathrm{eV}^{2}
$$

at $3 \sigma$, see Ref. 132 . Although this latter bound is stronger than the one in Eq. (3.3), it is not indicated whether it has been obtained after marginalizing over the atmospheric mixing angle or not. In any case, it seems clear that the previous bounds in Eqs. (3.3) and (3.4) have been derived assuming the same mass ordering for neutrinos and antineutrinos. Note that different mass orderings for neutrinos and antineutrinos would automatically imply CPT violation, even if the same value for the mass difference is obtained.

In the next section, we update the bounds from Eq. (3.3) using more recent oscillation data. At this point it is worth repeating that we are not considering any particular model of CPT violation and therefore all the results obtained can be regarded as model-independent. 


\subsection{Global fit to $\nu$ and $\bar{\nu}$ oscillation data}

In the light of the new experimental data, mainly from reactor and longbaseline accelerator experiments, we are going to update the bounds on CPT from neutrino oscillation data. Before, we have to perform two separate fits to neutrino and antineutrino oscillation data. For this analysis, we used the same data as was considered in the global fit to neutrino oscillations in Appendix A corresponding to version 1 of Ref. [2], uploaded to arXiv. Given that current atmospheric experiments, such as Super-Kamiokande [95], IceCube-DeepCore 93, 135 and ANTARES [94], can not distinguish neutrinos from antineutrinos event by event, we will not include them in this study. Let us summarize the neutrino samples considered, indicating in each case the neutrino or antineutrino parameters they are sensitive to

- solar neutrino data $70,72,74,76,81]: \theta_{12}, \Delta m_{21}^{2}, \theta_{13}$

- neutrino mode in long-baseline experiments K2K [107, MINOS [106, 132], $\mathrm{T} 2 \mathrm{~K}$ 103, 104 and $\mathrm{NO} \nu \mathrm{A}$ 110, 111]: $\theta_{23}, \Delta m_{31}^{2}, \theta_{13}$

- KamLAND reactor antineutrino data $\left[136\right.$ : $\bar{\theta}_{12}, \Delta \bar{m}_{21}^{2}, \bar{\theta}_{13}$

- short-baseline reactor antineutrino experiments Daya Bay 86, RENO 91 and Double Chooz 137: $\bar{\theta}_{13}, \Delta \bar{m}_{31}^{2}$

- antineutrino mode in long-baseline experiments ${ }^{2}$ MINOS $[106,132$ and T2K 103, 104: $\bar{\theta}_{23}, \Delta \bar{m}_{31}^{2} \bar{\theta}_{13}$

There is no reason to put bounds on $|\delta-\bar{\delta}|$ at the moment, since all possible values of $\delta$ or $\bar{\delta}$ are allowed. The exclusion of certain values of $\delta$ could only be obtained after combining neutrino and antineutrino data. In Fig. 3.2 we show the profiles for the neutrino (blue) and antineutrino (red) oscillation parameters obtained from the combined analyses of all relevant experiments for both cases. All parameters not plotted have been marginalized over. Note that, in some cases, the determination of the parameter is better for neutrinos and in other cases for antineutrinos. For example, the neutrino-reactor angle is much worse measured than its antineutrino counterpart, as can be seen in the upper right panel. This is of course due to the very good sensitivity of the current reactor experiments. This leads to a (weak) breaking in the degeneracy of the atmospheric angle, see the middle upper panel. The solar angle is better determined by neutrinos

\footnotetext{
${ }^{2}$ The K2K experiment took only data in neutrino mode. The NO $\nu \mathrm{A}$ experiment had taken only neutrino data when this analysis was performed.
} 

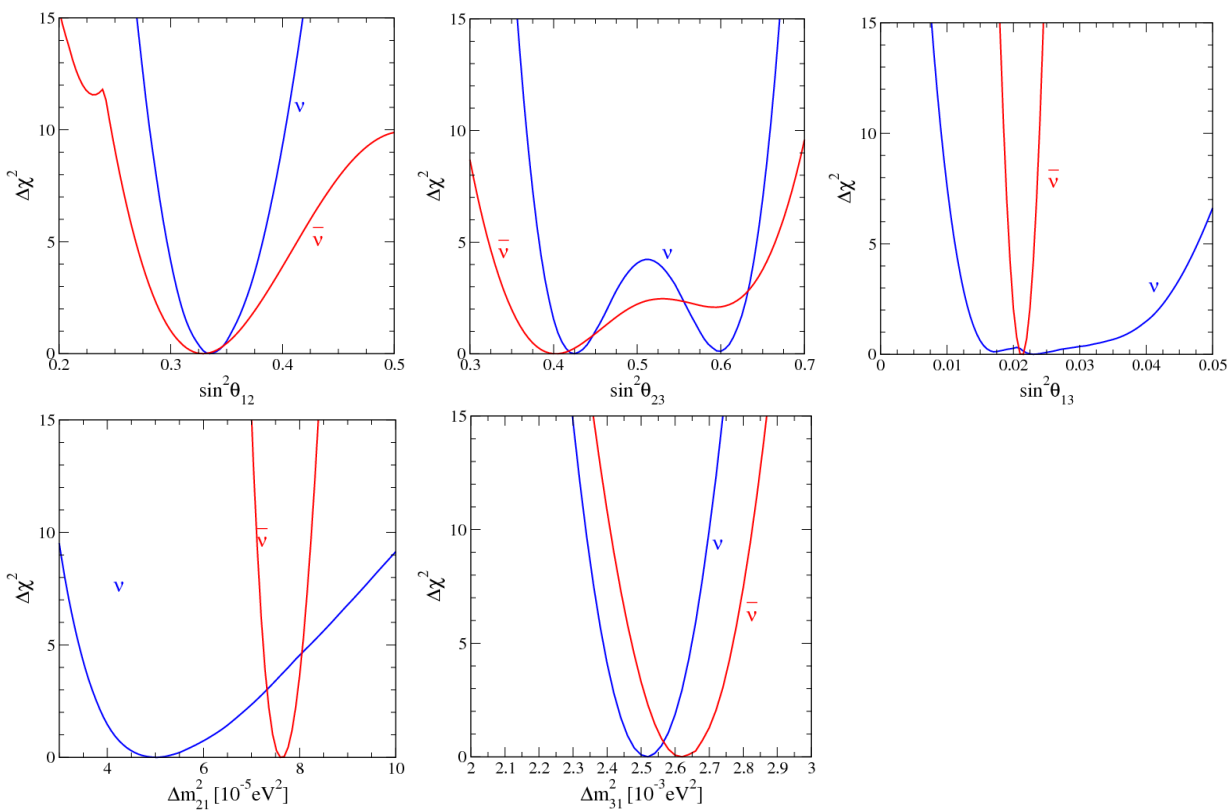

Figure 3.2: The profiles of the oscillation parameters obtained from a global fit to neutrino (blue) and antineutrino (red) data.

than antineutrinos (upper left panel), while the determination of the atmospheric mass splitting is equally good for neutrinos and antineutrinos. For the solar mass splitting we observe a tension due to a mismatch in the measurements of KamLAND and the solar experiments. This tension is already at the $2 \sigma$ level and if not resolved could hint towards new physics.

\subsection{Current bounds on CPT violation in the neutrino sector}

In this section we calculate the current bounds on CPT violation in the neutrino sector using the data discussed in the last section. We can use the profiles to calculate the CPT variables

$$
\Delta x=|x-\bar{x}|,
$$



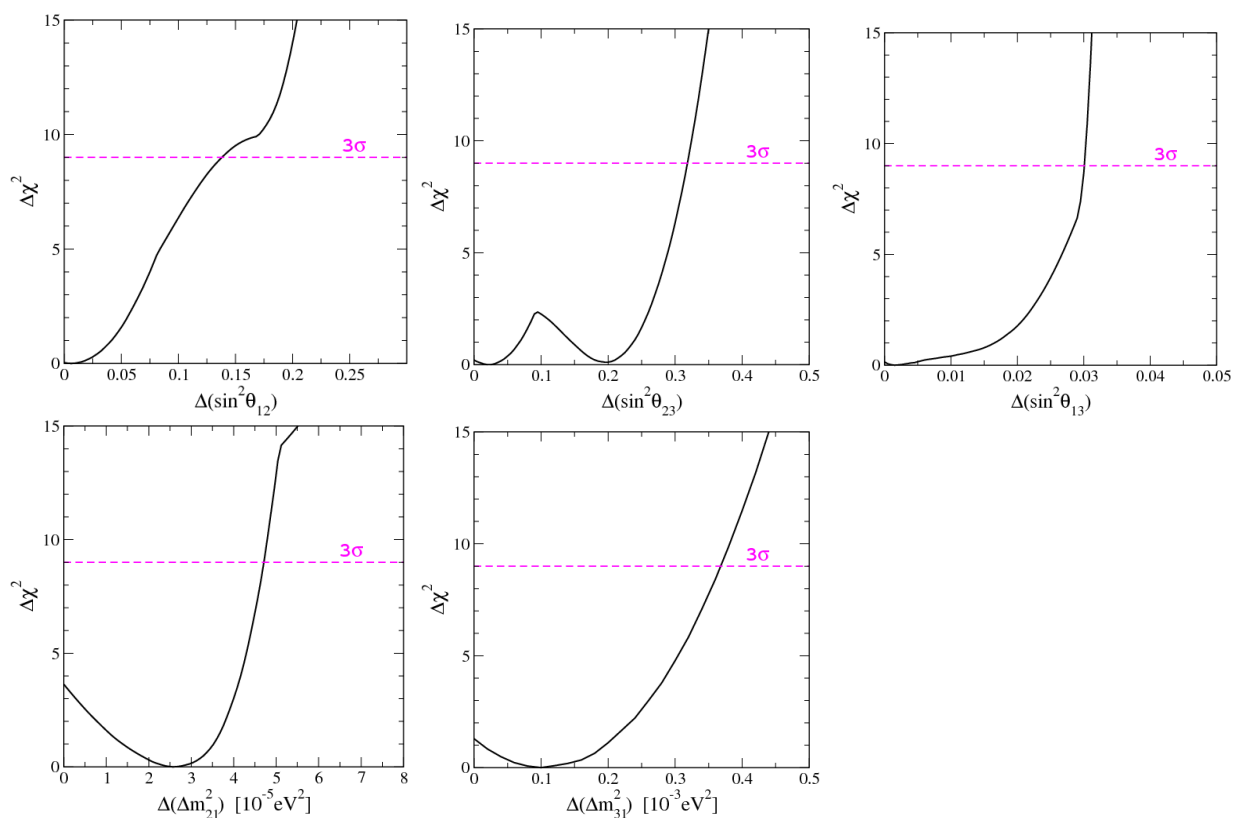

Figure 3.3: The profiles of the CPT violating variables.

where $x$ is any of the oscillation parameters. We assign to them the $\chi^{2}$-values

$$
\chi^{2}(\Delta x)=\chi^{2}(|x-\bar{x}|)=\min _{\substack{x, \bar{x} \\|x-\bar{x}|=\Delta x}}\left\{\chi^{2}(x)+\chi^{2}(\bar{x})\right\} .
$$

Doing this, we obtain the CPT profiles in Fig. 3.3 .

Hence, performing this exercise, we can read of the most up-to-date bounds on CPT violation to be

$$
\begin{aligned}
\left|\Delta m_{21}^{2}-\Delta \bar{m}_{21}^{2}\right| & <4.7 \times 10^{-5} \mathrm{eV}^{2}, \\
\left|\Delta m_{31}^{2}-\Delta \bar{m}_{31}^{2}\right| & <3.7 \times 10^{-4} \mathrm{eV}^{2}, \\
\left|\sin ^{2} \theta_{12}-\sin ^{2} \bar{\theta}_{12}\right| & <0.14 \\
\left|\sin ^{2} \theta_{13}-\sin ^{2} \bar{\theta}_{13}\right| & <0.03 \\
\left|\sin ^{2} \theta_{23}-\sin ^{2} \bar{\theta}_{23}\right| & <0.32,
\end{aligned}
$$

at $3 \sigma$ confidence level, improving the older bounds in Eqs. (3.3) and (3.4), except for $\sin ^{2} \theta_{13}$, that remains unchanged. Note that the limits on $\Delta\left(\Delta m_{31}^{2}\right)$ and $\Delta\left(\Delta m_{21}^{2}\right)$ are already better than the one of the neutral Kaon system and should 
be regarded as the best bounds on CPT violation on the mass squared so far. Note that the latter has a best fit point which is not zero, as already indicated in the last section. Here we can see that the aforementioned tension is indeed at $2 \sigma$ level. We observe a similar behavior for the atmospheric mass splitting although here it is much less pronounced. Another interesting result is the one for the atmospheric angle. We find a local minimum at the $\chi^{2}$ profile for $\Delta \sin ^{2} \theta_{23} \approx 0.2$, which arises as an artifact from the degeneracy observed in neutrino data in Fig. 3.2. This means that, if CPT is violated in nature with a difference of oscillation parameters at this value, we are not going to be able to observe it (at least at the moment). Note that the bound on $\Delta \sin ^{2} \theta_{13}$ is very weak, since the $3 \sigma$ exclusion is some $50 \%$ bigger than the best fit for antineutrinos itself. The only profile without any interesting features is the one for $\Delta \sin ^{2} \theta_{12}$.

It should be noted as well that, to obtain these bounds, we have assumed that neutrinos and antineutrinos have the same definition of $\Delta m^{2}$, i.e. the mass difference has the same sign. In principle, the mass difference in neutrinos and antineutrinos may have a different sign but, in this case one may argue that the sign difference is already a sign of CPT violation in itself.

We will come back to this topic in the context of the future experiment DUNE in chapter 6, where we will analyze if DUNE can further improve the bounds presented here. 


\section{Part II}

\section{The future}





\section{Chapter 4}

\section{Future oscillation experiments}

In the last chapters, we discussed the current status of neutrino oscillations in the standard picture and in some of its extensions. In the following chapters, we turn our attention to sensitivity studies for future experiments. Some of these sensitivity studies have been performed for standard oscillations, while others address certain models of physics beyond the standard model. Before turning to the main results, in the current chapter we will very briefly give an overview over most of the planned experiments, which will measure neutrino oscillations in the near future.

As we have seen in chapter 2, the combination of data from all current neutrino oscillation experiments leads to a precise oscillation picture. Many of the oscillation parameters are measured with good precision. The remaining unknowns are, basically, the octant of the atmospheric angle $\theta_{23}$ and the exact value of the phase $\delta$. Regarding the neutrino mass ordering, a $3.4 \sigma$ preference for normal ordering is obtained from the combination of all data. Note that this preference comes indeed from the global combination and that none of the current experiments can measure the mass ordering on its own. One may expect that measurements of current experiments will further improve, since they will still run for some time before the new experiments take over. However, it is not easy to predict the final results of current experiments, since the sensitivity is highly correlated to the true value of the $\mathrm{CP}$ phase $\delta$. The $\mathrm{NO} \nu \mathrm{A}$ experiment alone expects a $3 \sigma$ sensitivity for $30-50 \%$ of the values of $\delta$ by 2024 [138. If $\delta=3 \pi / 2$, the expected sensitivity would be higher than that and, then, a very strong result could be obtained by 2024 138. Note, however, that the $\mathrm{NO} \nu \mathrm{A}$ sensitivity analysis considers a fixed value of $\theta_{13}$. Upgrading T2K to T2K-II [139 will improve the sensitivity substantially, since the experiment should gather around $20 \times 10^{21}$ POT by 2026 , 
which would be roughly 6 times the current amount of data ${ }^{1}$ Combining beam data from $\mathrm{T} 2 \mathrm{~K}$ with atmospheric data from SK can improve the sensitivity even further, as shown in Ref. [95]. Performing a combined fit of T2K, $\mathrm{NO} \nu \mathrm{A}$ and eventually SK could bring the sensitivity to the $5 \sigma$ level within a few years. In any case, apart from the combinations of different experiments, a very robust determination of the neutrino mass ordering from a single current experiment seems rather unlikely. Indeed, one of the main goals of the next-generation neutrino oscillation experiments, including new long-baseline, reactor, and atmospheric neutrino detectors, will be to perform the determination of the mass ordering by a single experiment. The upcoming facilities will be able to measure the neutrino mass ordering with astonishing precision.

In the following we briefly discuss some of the proposed projects and their physics potential regarding neutrino oscillation parameters and neutrino mass ordering.

\section{Long-baseline experiments}

The Deep Underground Neutrino Experiment (DUNE) [43, 140 146] will be a new long-baseline accelerator experiment, with a small near detector and a huge far detector with a fiducial mass of $40 \mathrm{kton}$ located $1300 \mathrm{~km}$ away from the neutrino source at Fermilab. With its powerful 1.1 MW beam, it will be exposed to around $15 \times 10^{20}$ POTs (protons on target) per year, which will lead to a huge number of events and, therefore, to high precision measurements of the neutrino oscillation parameters. With the longest baseline ever for an accelerator neutrino experiment, DUNE will also be able to measure the neutrino mass ordering with a significance above $5 \sigma$ for any set of the oscillation parameters $\left(\theta_{23}, \delta\right)$ after 7 years of data taking. Note that this sensitivity could be further increased by using an improved energy reconstruction method, as shown in Ref. 147.

There are also plans to build a larger version of the Super-Kamiokande detector, Hyper-Kamiokande [148], that will be very similar to its predecessor but with a fiducial mass of 190 kton, 8.4 times larger than Super-Kamiokande. The Hyper-Kamiokande detector will be a requirement for the upgrade of T2K, the T2HK (Tokai-to-Hyper-Kamiokande) experiment [48. The very massive detector together with the upgraded neutrino beam with a target power of $1.3 \mathrm{MW}$ from J-PARC will guarantee a huge number of neutrino events and therefore larger statistics. As a consequence, T2HK will improve significantly the T2K measure-

\footnotetext{
${ }^{1}$ We are not aware of any study showing the T2K or SK expectations to the mass ordering in the next few years.
} 
ment of oscillation parameters and also will be able to determine the neutrino mass ordering after a few years of running time with very high significance.

In combination with atmospheric data from Hyper-Kamiokande, a $3 \sigma$ rejection of the wrong mass ordering would be expected after five years of data taking. A third project has been proposed as an extension of T2HK to Korea, the T2HKK (Tokai-to-Hyper-Kamiokande-and-Korea) experiment 149. This proposal includes a second far detector facility for the J-PARC neutrino beam, located at 1000-1300 $\mathrm{km}$ from the source. The longer path traveled within the Earth by the neutrinos detected in T2HKK will result in an enhanced sensitivity to the neutrino mass ordering if compared to T2HK alone.

The synergies and complementarities among the three long-baseline proposals above, DUNE, T2HK and T2HKK, have been discussed in Ref. [150]. It is found that the combination of their experimental results may significantly mitigate the limitations of a given experiment, improving the precision in both the determination of the mass ordering and the measurement of $\mathrm{CP}$ violation.

Note that, although here we have focused on the long-baseline side of DUNE and Hyper-Kamiokande, they are actually designed as multi-purpose experiments, with a rich physics program aiming to study the neutrino oscillations with accelerator, atmospheric (see chapter 5) and solar neutrinos [151] as well as to detect neutrinos from astrophysical sources and proton decay.

\section{Atmospheric experiments}

In atmospheric neutrino experiments, the sensitivity to the neutrino mass ordering comes from the matter effects that distort the pattern of neutrino oscillations inside the Earth. Based on the oscillatory pattern that depends on the reconstructed neutrino energy and zenith angle, an ideal experiment would observe a given number of events in each energy and zenith angle bin as shown in Fig. 4.1. Comparing the observed two-dimensional histograms with the theoretical ones for normal (left panel) or inverted ordering (right panel) allows to determine the true mass ordering that is realized in nature. In the following we list some of the future projects with this aim.

The Oscillation Research with Cosmics in the Abyss (ORCA) experiment 152 will be a large neutrino telescope placed deep inside the Mediterranean sea. It will detect the Cherenkov light emitted by the muons and electrons created by the interactions of atmospheric neutrinos in the sea. Unlike its precursor, ANTARES, with 12 lines and a separation of 70 meters between neighboring optical modules, ORCA will have 60 lines with modules separated by only 9 meters. Due to the matter effects on the propagation of atmospheric neutrinos, the ORCA 

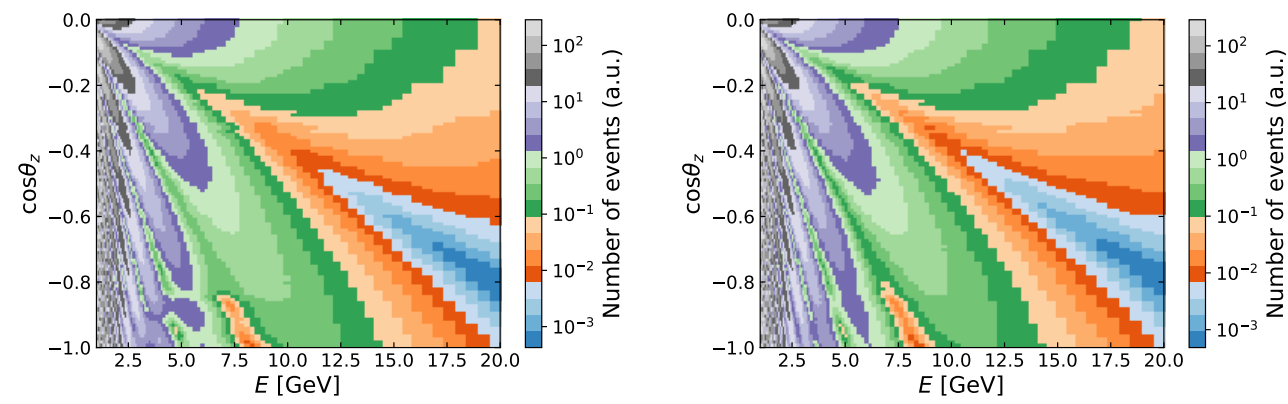

Figure 4.1: Expected number of events (arbitrary normalization) for an hypothetical atmospheric neutrino detector with perfect energy resolution as a function of the reconstructed neutrino energy $E$ and the cosine of the zenith angle $\cos \theta_{z}$, for normal (left) and inverted (right) ordering. Figure taken from Ref. [4].

experiment will be able to measure the neutrino mass ordering with very good precision. In particular, a $3 \sigma$ determination of the mass ordering can be expected after only three years of data taking, with even higher significance for the case in which nature has chosen normal ordering and the upper octant for the atmospheric mixing angle. Several studies have been performed in order to analyze the sensitivity of ORCA to the standard oscillation parameters [153,154 and also its potential to determine the Earth matter density through neutrino oscillation tomography 155 .

The current experiment IceCube aims at the detection of very high energy neutrinos using the antarctic ice as detection medium. Unfortunately, the energy threshold is above the relevant energy range for neutrino oscillations. However, the denser instrumented region DeepCore allows IceCube to decrease its energy threshold down to $E_{\text {th }}=6.3 \mathrm{GeV}$. A further improvement with an even denser zone, could lower $E_{\text {th }}$ to around $1 \mathrm{GeV}$. This is the goal of PINGU (Precision IceCube Next Generation Upgrade) [156]. With this very low-energy threshold, one of the main purposes of PINGU is the precise measurement of the atmospheric oscillation parameters and the determination of the neutrino mass ordering, with expected sensitivities similar to the ORCA experiment 2. Besides that, PINGU is expected to have the best sensitivity to $\nu_{\tau}$ appearance and to determine accurately the octant of the atmospheric mixing angle.

\footnotetext{
${ }^{2}$ The effect of statistic and systematic uncertainties on the PINGU sensitivity to the mass ordering has been presented in Ref. 157.
} 
The India-based Neutrino Observatory (INO) is a very ambitious project, aiming to detect atmospheric neutrinos with a 50 kton magnetized iron calorimeter (ICAL) [158]. The most outstanding feature of the INO experiment will be its capability to distinguish neutrinos from antineutrinos in an event by event basis. As a result, the identification of the matter effects in the neutrino and antineutrino propagation will be much cleaner in comparison with the sea water/ice Cherenkov detectors. Indeed, one of the main scientific goals of INO will be the determination of the neutrino mass ordering [159. According to the Physics White Paper of the ICAL (INO) Collaboration 158, after 10 years run, INO will be able to identify the correct neutrino mass ordering with a significance larger than $3 \sigma$. Note that, because of the neutrino and antineutrino separation, INO could be used to measure CPT violation as well, which is not possible with other atmospheric neutrino experiments, as explained in chapter 3

\section{Medium-baseline reactor experiments}

We have focused so far on extracting the neutrino mass ordering from matter effects in the neutrino propagation through the Earth's interior. An alternative technique is that provided by medium-baseline reactor neutrino experiments 160 . For baselines of the order of $50 \mathrm{~km}$, the survival probability for reactor antineutrinos exhibits a pattern that may allow the discrimination between normal and inverted mass orderings. Indeed, for such distances, the electron antineutrino survival probability is given by the following expression:

$$
\begin{aligned}
P_{\bar{\nu}_{e} \rightarrow \bar{\nu}_{e}}=1 & -\cos ^{4} \theta_{13} \sin ^{2} 2 \theta_{12} \sin ^{2} \Delta_{21} \\
& -\sin ^{2} 2 \theta_{13}\left[\sin ^{2} \Delta_{31}+\sin ^{2} \theta_{12} \sin ^{2} \Delta_{21} \cos 2 \Delta_{31}\right. \\
& \left.\mp \frac{\sin ^{2} \theta_{12}}{2} \sin 2 \Delta_{21} \sin 2\left|\Delta_{31}\right|\right]
\end{aligned}
$$

where $\Delta_{i j}=\frac{\Delta m_{i j}^{2} L}{4 E}$ and the minus (plus) sign in the last term corresponds to normal (inverted) mass ordering. This probability contains a main oscillatory term with a frequency given by the solar neutrino mass splitting $\Delta m_{21}^{2}$, plus an additional term whose frequency depends on the sign of the atmospheric splitting $\Delta m_{31}^{2}$, i.e. on the neutrino mass ordering. The effect of the ordering over the neutrino survival probability in a medium-baseline reactor experiment is illustrated in Figure 4.2. The black (red) line corresponds to the full neutrino survival probability for normal (inverted) mass ordering. This plot was obtained using the best-fit values from Table 2.2 for each ordering. Note that, to achieve 


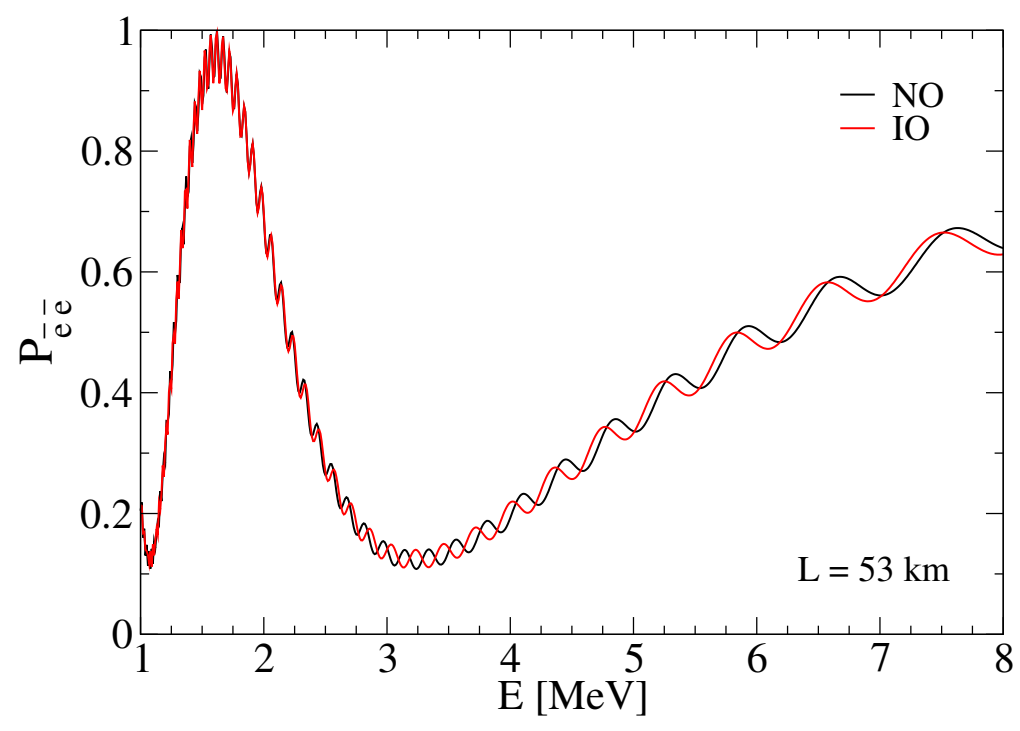

Figure 4.2: Electron antineutrino survival probabilities in a medium-baseline reactor experiment with $L=53 \mathrm{~km}$. The black (red) line corresponds to normal (inverted) mass ordering using the best-fit values from Table 2.2

this resolution of frequencies, an excellent knowledge of the relevant oscillation parameters is necessary.

The Jiangmen Underground Neutrino Observatory (JUNO) [161] is a 20 kton multi-purpose underground liquid scintillator detector. The site of the experiment, located $53 \mathrm{~km}$ away from the Yangjiang and Taishan nuclear power plants in China, was chosen to optimize its sensitivity to the neutrino mass ordering, one of its main physics goals. Like any other reactor neutrino experiment, JUNO will be sensitive to the disappearance of electron antineutrinos, with about $10^{5}$ events expected after six years of run time. From this high-statistics data sample, JUNO will try to reconstruct with extremely good precision the neutrino oscillation spectrum and to discriminate the different high-frequency behavior for normal and inverted mass ordering, as illustrated in Eq. (4.1) and Fig. 4.2. For a projected energy resolution of $3 \%$ at $1 \mathrm{MeV}$, JUNO will be able to establish the neutrino mass ordering at the level of $3-4 \sigma$ in 6 years. Apart from the mass ordering, JUNO will also provide precision measurements of the oscillation parameters $\theta_{12}$, $\Delta m_{21}^{2}$ and $\Delta m_{31}^{2}$ with an accuracy of below $1 \%$ for each of them. In this sense, JUNO might help to solve the observed disagreement between the mass splitting measured at solar experiments and at the reactor experiment KamLAND. If the 
discrepancy persists after new measurements by JUNO and future solar results by Super(Hyper)-Kamiokande, it could be considered as an indication for new physics 162 . 



\section{Chapter 5}

\section{Standard neutrino oscillations at DUNE}

The search for neutrino oscillations at the upcoming long baseline experiments, such as DUNE, will play a key role in the agenda of neutrino physics experimentation over the coming decades 140,142 . The new generation of experiments will be able to substantially improve our current measurement of the $\theta_{23}$ angle, the atmospheric mass splitting $\Delta m_{31}^{2}$ and its sign (the neutrino mass ordering), and can potentially provide a precise measurement of $\delta$. Therefore, DUNE might resolve all the remaining issues in the 3 neutrino paradigm, addressed in chapter 2. The capability of DUNE to tackle these problems is the topic of this chapter. After giving some details on the simulation of DUNE in Sec. 5.1, we focus shortly on the determination of oscillation parameters with DUNE in Sec. 5.2 and then show in Sec. 5.3 how DUNE will be able to measure the neutrino mass ordering using atmospheric neutrinos, which will give an extra bonus to the total sensitivity of the experiment.

\subsection{Simulation of the DUNE experiment}

In order to simulate the long-baseline experiment DUNE, introduced in the last chapter, we use the GLoBES package [108, 109 together with the auxiliary file presented in Ref. 163. In general, our simulations of DUNE consider a period of 3.5 years running time in both neutrino and antineutrino mode, taking into account the disappearance and appearance channels for neutrinos and antineutrinos. Following Refs. [140] and [163, we include several types of background events. These are due to the misinterpretation of neutrinos as antineutrinos and 
vice-versa, contamination of electron neutrinos and antineutrinos in the beam, misinterpretation of muon as electron neutrinos, as well as the appearance and misinterpretation of tau neutrinos and neutral current interactions. We associate to each of the backgrounds a nuisance normalization parameter, ranging between $5 \%$ and $20 \%$, over which we later marginalize. In addition, we assign a $2 \%$ error on the signals in the appearance channels and a $5 \%$ error in the disappearance channels, as indicated in the studies performed by the DUNE Collaboration in Ref. 140. We generate future DUNE data (fake data) using some set of parameters $\overrightarrow{p_{\text {true }}}$ and define the $\chi^{2}$ function

$$
\chi^{2}(\vec{p})=\min _{\vec{\alpha}} \sum_{\text {channels }} 2 \sum_{n}\left[N_{n}^{\text {test }}-N_{n}^{\text {dat }}+N_{n}^{\text {dat }} \log \left(\frac{N_{n}^{\text {dat }}}{N_{n}^{\text {test }}}\right)\right]+\sum_{i}\left(\frac{\alpha_{i}}{\sigma_{i}}\right)^{2},
$$

where $\vec{p}$ denotes some set of neutrino oscillation parameters. Here $N_{n}^{\text {dat }}$ corresponds to the simulated event number in the $n$-th bin using $\vec{p}_{\text {true }}, N_{n}^{\text {test }}$ is the event number in the $n$-th bin associated to the parameters $\vec{p}$ and $\alpha_{i}$ and $\sigma_{i}$ are the nuisance parameters and their corresponding standard deviations, respectively. Note that $N_{n}^{\text {test }}$ also depends on $\vec{\alpha}$, since these can change the number of signal and background events.

\subsection{Beam determination of oscillation parameters}

Following the description of the last section, we can perform a simulation of DUNE assuming standard neutrino oscillations. We create the fake data using the best fit parameter from the global analysis of chapter 2. Next, we try to reconstruct this spectrum varying the oscillation parameters. We keep the solar parameters fixed throughout this simulation, because their effect is negligible in long baseline experiments. The rest of the parameters are varied. We perform one fit with the reactor angle free and another one adding a penalization due to the measurement of current reactor neutrino experiments. The results of these fits are shown in Fig. 5.1. Note that the variables not shown are always marginalized over. We also show for comparison the regions allowed by current data. We see that the determination of $\delta$ and $\Delta m_{31}^{2}$ is mostly independent of $\theta_{13}$. The sensitivity to the atmospheric angle $\theta_{23}$ on the other hand improves considerably once imposing the prior. This behavior can be appreciated also in Fig. 5.2, where we plot the $\chi^{2}$ profiles for each parameter. In general, one sees that DUNE will improve the measurement of $\Delta m_{31}^{2}, \delta$ and $\theta_{23}$. In combination with the results 


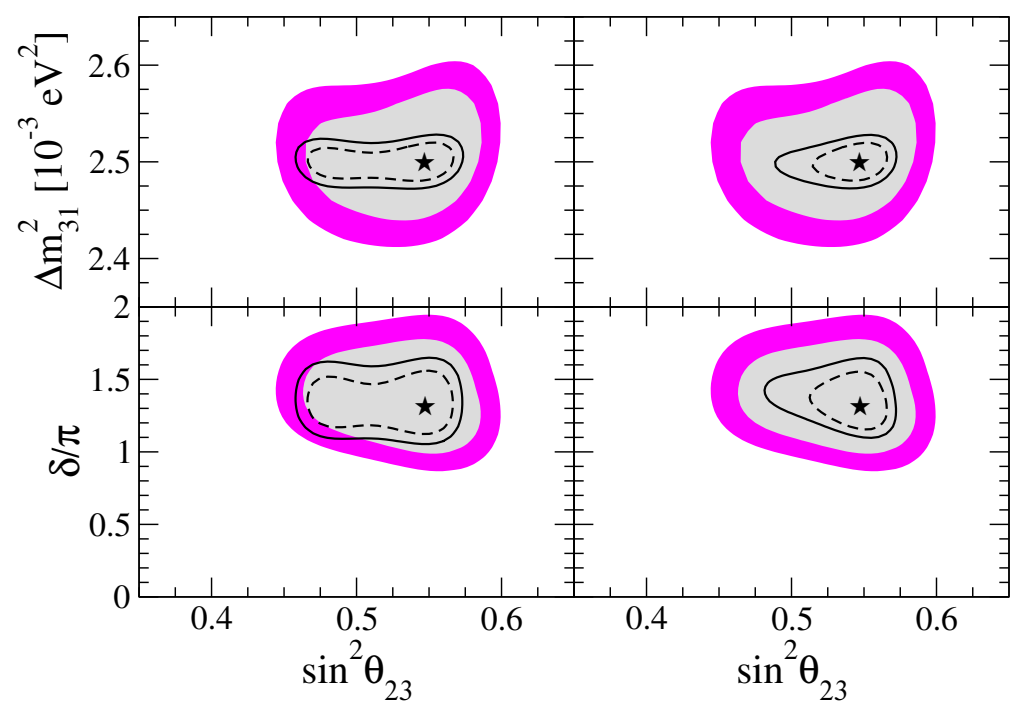

Figure 5.1: Sensitivity of DUNE (black lines) to measure some of the oscillation parameters in comparison with the current knowledge (filled regions) at 90 and 99\% confidence level. The left panels use only DUNE, while the right panels show the results after imposing a prior on $\theta_{13}$ in the analysis.

expected from the medium baseline reactor experiment JUNO, all of the neutrino oscillation parameters will be measured with high precision.

\subsection{Mass ordering determination with atmospheric neutrinos}

The future long baseline facility DUNE [65 67] aims to extract the sign of the atmospheric mass splitting and the $\mathrm{CP}$ violating phase $\delta$ through the oscillation channels $\nu_{\mu} \rightarrow \nu_{e}$ and $\bar{\nu}_{\mu} \rightarrow \bar{\nu}_{e}$, the same channels exploited by the current $\mathrm{T} 2 \mathrm{~K}[164$ and $\mathrm{NO} \nu \mathrm{A}[102]$ experiments. However, both quantities can also be extracted using atmospheric neutrino beam $₫$. The idea of using atmospheric neutrino fluxes to distinguish the type of mass ordering is well-known in the literature since a long time $[171-173$. Indeed, as discussed in Chapter 2, a preference at the $2 \sigma$ level in favor of normal ordering has been obtained at SuperKamiokande [95]. These pioneer studies focused mostly on muon calorimeter

\footnotetext{
${ }^{1}$ See Refs. $165 \quad 169$ and the recent work of 170 for a CP violation measurement using sub-GeV atmospheric neutrinos.
} 

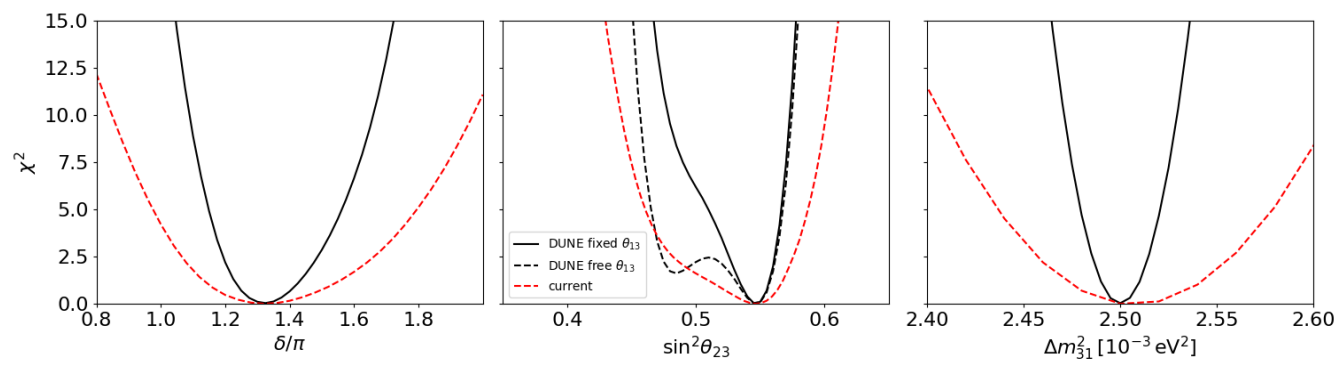

Figure 5.2: The $\chi^{2}$ profiles for $\delta$ (left), $\sin ^{2} \theta_{23}$ (center) and $\Delta m_{31}^{2}$ (right). In the central panel we plot the profile obtained from the analysis of DUNE with and without a prior on the reactor angle $\theta_{13}$. For comparison we also plot in red the current profiles, as obtained in chapter 2 .

detectors, such as MONOLITH 174, MINOS [175] or INO in which the muon charge can be determined, see also Refs. $159,176,188$.

Neutrino observatories can also extract the sign of the atmospheric mass difference with lower energy detection thresholds for atmospheric neutrino extensions by looking at the less sensitive but higher statistics muon disappearance channels $\nu_{\mu} \rightarrow \nu_{\mu}$ and $\bar{\nu}_{\mu} \rightarrow \bar{\nu}_{\mu}$ [189. The IceCube collaboration has recently reported a preference for NO with a p-value of $p_{\mathrm{IO}}=15.3 \%$ for the IO hypothesis 190 using data collected by the DeepCore extension. This will also be the main target for ORCA [152, 191] and PINGU 156, 157, 192, 193, see e.g. 117, 154, 168, 194,198 .

Here we exploit the atmospheric neutrino signatures at the DUNE detector, a Liquid Argon Time Projection Chamber (LArTPC). Despite this detection technology, in the absence of a magnetic field, does not allow for a charge identification of the final lepton state, one can make use of a particular event topology available in argon detectors: muon capture 199 . This bonus process will provide a clean measurement of the muon charge, that will considerably improve the capabilities of DUNE to perform mass ordering measurements with atmospheric neutrinos. Notice that the advantage is twofold, as (i) measurements of the mass ordering could be available before the beam starts, and (ii) the combination with the beam information will enhance the expected sensitivity reach. We shall show that muon capture events could greatly enhance the sensitivity to the mass ordering from atmospheric neutrinos only. For an earlier, and preliminary, appraisal of the neutrino mass ordering sensitivity in DUNE using atmospheric neutrinos, including a statistical discrimination between neutrinos and antineutrinos, see Ref. [140. The latter work was largely based on previous studies in the framework of the 
LBNE project, see 200. The results of the following sections are published in Ref. [10].

\subsubsection{Matter effects and atmospheric neutrinos}

In atmospheric neutrino experiments, the size of matter effects is given by the effective mixing angle $\theta_{13}$ in matter, which leads to the golden channel transitions $\nu_{\mu} \rightarrow \nu_{e}, \nu_{e} \rightarrow \nu_{\mu}, \bar{\nu}_{\mu} \rightarrow \bar{\nu}_{e}$ and $\bar{\nu}_{e} \rightarrow \bar{\nu}_{\mu}$ and reads, within the simple two-flavor mixing framework and for constant matter density, as

$$
\sin ^{2} 2 \theta_{13}^{\mathrm{m}}=\frac{\sin ^{2} 2 \theta_{13}}{\sin ^{2} 2 \theta_{13}+\left(\cos 2 \theta_{13} \mp \frac{A}{\Delta m_{31}^{2}}\right)^{2}},
$$

where the minus (plus) sign refers to neutrinos (antineutrinos). The matter potential is given by $A=2 \sqrt{2} G_{F} N_{e} E$ and $N_{e}$ is the electron number density in the Earth's interior. Consequently, matter effects will enhance (deplete) the neutrino (antineutrino) oscillation probabilities $P\left(\nu_{\mu} \rightarrow \nu_{e}\right)$ and $P\left(\nu_{e} \rightarrow \nu_{\mu}\right)$ $\left(P\left(\bar{\nu}_{\mu} \rightarrow \bar{\nu}_{e}\right)\right.$ and $\left.P\left(\bar{\nu}_{e} \rightarrow \bar{\nu}_{\mu}\right)\right)$ if the mass ordering is normal. When the resonance condition

$$
\Delta m_{31}^{2} \cos 2 \theta_{13}=2 \sqrt{2} G_{F} N_{e} E
$$

is satisfied, matter effects are expected to have their largest contribution. In the case of atmospheric neutrinos, which travel distances of several thousand of kilometers, and for $\Delta m_{31}^{2} \sim 2.5 \times 10^{-3} \mathrm{eV}^{2}[2]$, the resonance condition will take place at neutrino energies $\sim 3-8 \mathrm{GeV}$, depending on the precise value of $N_{e}$ in the Earth's interior and, in the case of core crossing neutrinos, also on the exact path length.

Matter effects are also marginally present in the muon disappearance channels $P\left(\nu_{\mu} \rightarrow \nu_{\mu}\right)$ and $P\left(\bar{\nu}_{\mu} \rightarrow \bar{\nu}_{\mu}\right)$, relevant for both long-baseline and atmospheric neutrino beams. In the simplified case of a constant matter density, the disappearance probability at terrestrial baselines is given by

$$
\begin{aligned}
P\left(\stackrel{(-)}{\nu}_{\mu} \rightarrow \stackrel{(-)}{\nu}\right) & =1-\cos ^{2} \theta_{13}^{\mathrm{m}} \sin ^{2} 2 \theta_{23} \times \sin ^{2}\left[1.27\left(\frac{\Delta m_{31}^{2}+A+\left(\Delta m_{31}^{2}\right)^{\mathrm{m}}}{2}\right) \frac{L}{E}\right] \\
& -\sin ^{2} \theta_{13}^{\mathrm{m}} \sin ^{2} 2 \theta_{23} \times \sin ^{2}\left[1.27\left(\frac{\Delta m_{31}^{2}+A-\left(\Delta m_{31}^{2}\right)^{\mathrm{m}}}{2}\right) \frac{L}{E}\right] \\
& -\sin ^{4} \theta_{23} \sin ^{2} 2 \theta_{13}^{\mathrm{m}} \sin ^{2}\left[1.27\left(\Delta m_{31}^{2}\right)^{\mathrm{m}} \frac{L}{E}\right]
\end{aligned}
$$


where $\theta_{13}^{\mathrm{m}}$ is that of Eq. $(5.2)$ and

$$
\left(\Delta m_{31}^{2}\right)^{\mathrm{m}}=\Delta m_{31}^{2} \sqrt{\sin ^{2} 2 \theta_{13}+\left(\cos 2 \theta_{13} \mp \frac{A}{\Delta m_{31}^{2}}\right)^{2}} .
$$

The muon survival probabilities will be suppressed (enhanced) if the ordering is normal (inverted), so the effect is opposite to the one present in the $\nu_{e} \rightarrow \nu_{\mu}$ oscillation channel. Therefore, when dealing with atmospheric neutrino beams, since there is an irreducible muon neutrino background from $\nu_{e} \rightarrow \nu_{\mu}$ oscillations, the size of the matter effects will be reduced. The distance $L$ traveled through the Earth by these atmospheric neutrino beams is fixed by their arrival zenith angle $\theta_{z}$ (with $\cos \theta_{z}=1$ for vertical down-going neutrinos and $\cos \theta_{z}=-1$ for vertical up-going neutrinos),

$$
L=R_{\oplus}\left(\sqrt{\left(1+\frac{h}{R_{\oplus}}\right)^{2}-\left(1-\cos \theta_{z}\right)^{2}}-\cos \theta_{z}\right),
$$

with $R_{\oplus}$ the Earth's radius and $h \simeq 15 \mathrm{~km}$ the neutrino production distance from the Earth's surface. The dependence of the survival probabilities $P\left(\nu_{\mu} \rightarrow \nu_{\mu}\right)$ and $P\left(\bar{\nu}_{\mu} \rightarrow \bar{\nu}_{\mu}\right)$ on the neutrino energy $E$ and the cosine of the zenith angle, $\cos \theta_{z}$, is shown in the left and right panels of Fig. 5.3 for normal and inverted ordering (top and bottom figures). Notice that, in the case of normal ordering, the resonance takes place at the aforementioned energies $(3-8 \mathrm{GeV})$ for almost vertical up-going neutrinos, $-1<\cos \theta_{z}<-0.8$, while for the inverted ordering, such a resonant enhancement in the transition probabilities will take place in the antineutrino channel instead. Therefore, even if both the angular and the energy resolution of the detector should be optimal, the key ingredient to disentangle matter effects (and, ultimately, the neutrino mass ordering) is to have a detector with muon charge tagging, generally achieved with a magnetized detector. However, as we shall shortly see, LArTPCs allow for such a possibility without the need of a magnetic field.

\subsubsection{Atmospheric neutrino events in DUNE: muon capture in argon}

Our statistical analyses will deal with three possible fully contained event samples at atmospheric neutrino detectors: $\mu^{-}$-like events that undergo muon 

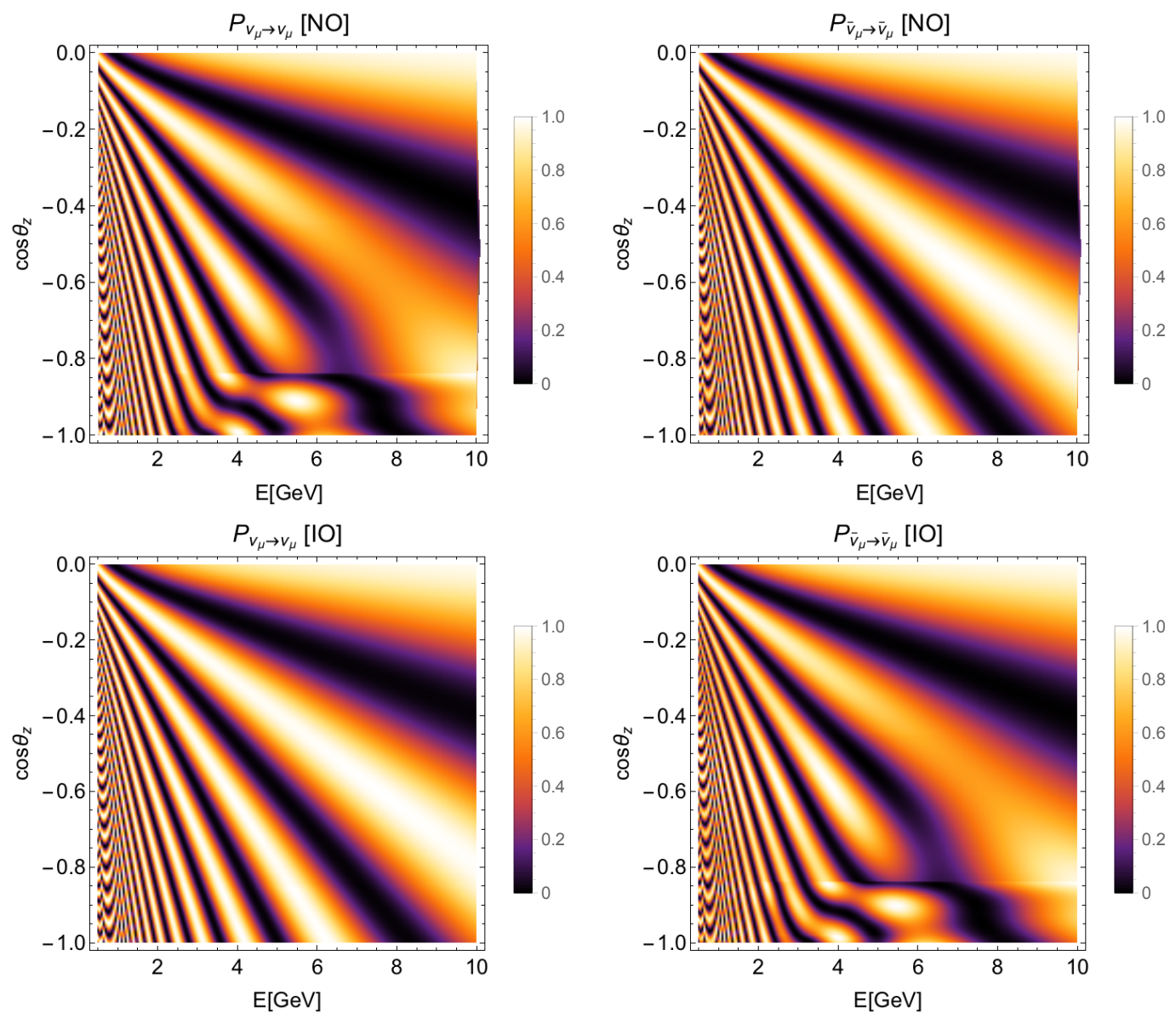

Figure 5.3: Left panels: survival probability $P\left(\nu_{\mu} \rightarrow \nu_{\mu}\right)$ as a function of the neutrino energy $E$ and the cosine of the zenith angle, $\cos \theta_{z}$, for normal (inverted) ordering in the top (bottom) line. Right panels: same as in the left panels, but for the antineutrino channel. Figure taken from Ref. 10 . 
capture $\left(N_{i, j, \mu}^{\mathrm{cap}}\right)$, the rest of the muons and all of the antimuons that undergo muon decay $\left(N_{i, j, \mu}^{\text {rest }}\right)$, and $e$-like events $\left(N_{i, j, e}\right){ }^{2}$,

Let us start with the $\mu^{-}$-like contained events produced by the interactions of atmospheric up-going neutrinos in the LArTPC DUNE detector. In a LArTPC, both ionization charge 201,202 and scintillation light 203 information can be used to infer the neutrino/antineutrino content in a muon neutrino beam. This is possible by exploiting the signature of $\mu^{-}$capture on argon nuclei, only available for contained events. In argon, the effective $\mu^{-}$lifetime resulting from the competing decay and nuclear capture processes is given by:

$$
\tau=\left(1 / \tau_{\text {cap }}+Q / \tau_{\text {free }}\right)^{-1}
$$

where $\tau_{\text {cap }}$ is the lifetime of the capture process, $Q=0.988$ is the Huff correction factor [204 205], which is a small correction taking into account the fact that the normal muon decay rate is reduced for a bound muon, and $\tau_{\text {free }}=2197.0 \mathrm{~ns}[206$ is the muon lifetime in vacuum. The resulting $\mu^{-}$capture fraction is then given by:

$$
\epsilon^{\text {cap }}=\tau / \tau_{\text {cap }}=1-\tau / \tau_{\text {free }}
$$

The most precise determination of the $\mu^{-}$lifetime in argon was obtained in 207, $\tau=(616.9 \pm 6.7) \mathrm{ns}$, resulting in

$$
\epsilon^{\text {cap }}=(71.9 \pm 0.3) \%
$$

This measurement is fully compatible with the earlier measurement of $\tau=(606 \pm$ 29) ns in 205 and the preliminary result from LArIAT of $\tau=(626 \pm 48) \mathrm{ns} 208$. In our analysis, we use the central value and uncertainty in Eq. 5.9$)^{3}$

For our sensitivity estimates, we also assume a $100 \%$ efficiency for tagging Michel electrons and positrons from $\mu^{ \pm}$decays at rest, as done in 200. Any tagging inefficiency would cause decay events to be mis-interpreted as capture events, and should therefore be avoided for optimal muon neutrino/antineutrino separation. We consider this approximation to be sufficient for the purposes of this feasibility study. Efficiency estimates using detailed DUNE simulations are not yet publicly available. Still, early data from ICARUS 210 and LArIAT 208 have already shown that the Michel electron tagging efficiency can reach values close to unity in LArTPC detectors using either charge or light information. In any case, any Michel electron tagging inefficiency smaller than $\left(1-\epsilon^{\text {cap }}\right) \simeq 28 \%$

\footnotetext{
${ }^{2}$ Electron charge identification is impossible at $\mathrm{GeV}$ energies and we shall consider just one event sample which accounts for both $e^{+}$and $e^{-}$-like events.

${ }^{3}$ Note that the first measurement of the capture rate was performed in Ref. 209].
} 



Figure 5.4: The event topologies of capture (left) and decay events as observed in the argon detectors ArgoNeuT and $\mu$ BooNE, respectively. Figures taken from Refs. 201,211].

will have a sub-dominant contribution to the mixing of muon neutrino and muon antineutrino stopping samples in our analysis, compared to the effect of $\mu^{-}$tracks that do not capture and decay. Note that captured muons will also decay into electrons. This, however, happens only after some time, making the distinction possible. Fig. 5.4 depicts the different topologies expected for capture events (left) and decay events (right). These figures correspond to real events in argon detectors; ArgoNeuT on the left [201] and $\mu$ BooNE on the right [211].

Therefore, it appears possible to select a statistically significant, highly pure, sample of $\mu^{-}$-like atmospheric neutrino interactions, with an identification efficiency of $\epsilon^{\text {cap }}$, as given in Eq. (5.9). The number of muon-like contained events in the $i$-th neutrino energy $\left(E_{r}\right)$ and $j$-th cosine of the zenith angle $\left(c_{r, \nu}\right)$ bin (both reconstructed quantities) reads as

$$
\begin{aligned}
& N_{i, j, \mu^{-}\left(\mu^{+}\right)}=\frac{2 \pi N_{\mathrm{T}} t}{V_{\operatorname{det}}} \int_{E_{r, i}}^{E_{r, i+1}} d E_{r, \nu} \int_{c_{r, \nu, j}}^{c_{r, \nu, j+1}} d c_{r, \nu} \int_{0}^{\infty} d E_{\nu} \int_{-1}^{1} d c_{\nu} V_{\mu}
\end{aligned}
$$

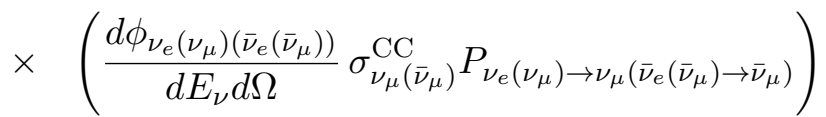

$$
\begin{aligned}
& \times R_{e}^{\mu}\left(E_{r, \nu}, E_{\nu}\right) R_{\theta}^{\mu}\left(\theta_{r, \nu}, \theta_{\nu}\right),
\end{aligned}
$$

where $d \phi_{\nu}$ 's are the atmospheric neutrino differential fluxes, $\sigma^{\mathrm{CC}}$ is the $\mathrm{CC}$ neutrino cross sections in argon, $N_{\mathrm{T}}$ is the number of available targets, $V_{\text {det }}$ is the total volume of the detector, $V_{\mu}$ is the effective detector volume and $t$ is the exposure time. Finally, $R_{e}^{\mu}\left(E_{r, \nu}, E_{\nu}\right)$ and $R_{\theta}^{\mu}\left(\theta_{r, \nu}, \theta_{\nu}\right)$ account for the energy and angular smearing. 



Figure 5.5: Number of expected muon events after $400 \mathrm{kt} \cdot \mathrm{yr}$ exposure time, separating capture and decay events (first two panels) and combining all muon type events (right panel), using the parameters in Tab.5.3 and $\sin ^{2} \theta_{23}=0.547$. Figure taken from Ref. 10 .

The $\mu^{-}$-like contained events that undergo muon capture are given by

$$
N_{i, j, \mu}^{\mathrm{cap}}=\epsilon^{\mathrm{cap}} N_{i, j, \mu^{-}},
$$

while the remaining muon-like events are given by

$$
N_{i, j, \mu}^{\mathrm{rest}}=\left(1-\epsilon^{\mathrm{cap}}\right) N_{i, j, \mu^{-}}+N_{i, j, \mu^{+}} .
$$

In Fig. 5.5 we show an example for the expected number of events, fixing the value the oscillation parameters to the ones in Tab. 5.3 and the atmospheric mixing angle to $\sin ^{2} \theta_{23}=0.547$. In the first two panels, we plot the capture and decay events separately, respectively, while showing the combination of both samples in the right panel. Note that when trying to reconstruct the right panel there can be more degeneracies among parameters in the analysis than when fitting the two sets independently and, therefore, one expects to obtain stronger results. This is indeed the case, as we will see below.

In the case of electrons, the number of $e$-like events in the $i$-th and $j$-th bin in $\left(E_{r}, c_{r, \nu}\right)$ reads as

$$
\begin{aligned}
N_{i, j, e^{-}\left(e^{+}\right)} & =2 \pi N_{\mathrm{T}} t \int_{E_{r, i}}^{E_{r, i+1}} d E_{r, \nu} \int_{c_{r, \nu, j}}^{c_{r, \nu, j+1}} d c_{r, \nu} \int_{0}^{\infty} d E_{\nu} \int_{-1}^{1} d c_{\nu} \\
& \times\left(\frac{d \phi_{\nu_{e}\left(\nu_{\mu}\right)\left(\bar{\nu}_{e}\left(\bar{\nu}_{\mu}\right)\right)}}{d E_{\nu} d \Omega} \sigma_{\nu_{e}\left(\bar{\nu}_{e}\right)}^{\mathrm{CC}} P_{\nu_{e}\left(\nu_{\mu}\right) \rightarrow \nu_{e}\left(\bar{\nu}_{e}\left(\bar{\nu}_{\mu}\right) \rightarrow \bar{\nu}_{e}\right)}\right) \\
& \times R_{e}^{e}\left(E_{r, \nu}, E_{\nu}\right) R_{\theta}^{e}\left(\theta_{r, \nu}, \theta_{\nu}\right) .
\end{aligned}
$$


As previously stated, we just consider one electron-like event sample $N_{i, j, e}$ which is computed as the sum of $N_{i, j, e^{-}}$and $N_{i, j, e^{+}}$.

Regarding the atmospheric electron and muon (anti) neutrino fluxes, for the differential fluxes $\frac{d \phi_{\nu \alpha}}{d E_{\nu} d \Omega}$ that appear in Eqs. $(\sqrt{5.10})$ and $((5.13))$, we use the results from Ref. 212], albeit very similar numbers would have been obtained using the fluxes from Refs. [98, 213,214]. We shall comment in the following section on the errors on these atmospheric neutrino fluxes, that have been properly added to other sources of systematic uncertainties in our numerical studies.

The cross sections for muon and electron (anti)neutrino interactions on argon nuclei in the $0-10 \mathrm{GeV}$ neutrino energy range have been simulated by means of the GENIE Monte Carlo neutrino event generator [215. GENIE is extensively used by the neutrino physics community and by the DUNE Collaboration in particular. As our cross section model, we use the total charged-current (anti)neutrino cross sections provided by GENIE version 2.12 .10 on ${ }^{40} \mathrm{Ar}$ nuclei (18 protons and 22 neutrons). The model accounts for a comprehensive list of interaction processes, including quasi-elastic scattering, baryon resonance production, coherent pion production in neutrino-nucleus scattering and deep inelastic scattering. Nuclear effects affecting total cross sections are included. Final state hadronic interactions occurring within the argon target nucleus are not simulated, but indirectly accounted for via our assumed energy and angular resolution functions.

To compute the effective volume fraction $V_{\mu} / V_{\text {det }}$ in Eq. (5.10) for contained muon events, we have approximated the DUNE detector to be made of four independent modules with approximately 13 kton of LAr active mass each, each of them assumed to have an elliptical cylindrical shape with a height of $h=12 \mathrm{~m}$ and major and minor semi-axis of $a=29 \mathrm{~m}$ and $b=7.25 \mathrm{~m}$, respectively. For the calculation of the effective volume we have taken into account the muon range 216 in argon $R_{\mu}\left(E_{\mu}\right)$, which depends on the lepton energy. It generalizes the formulas in Refs. 189, 216 to an elliptic base and is given by

$$
V_{\mu}=4 \times 2 a b h \arcsin \left(1-\frac{R_{\mu}^{2}\left(E_{\mu}\right)}{D_{b}^{2}} \sin ^{2} \theta_{\nu}\right)\left(1-\frac{R_{\mu}\left(E_{\mu}\right)}{h}\left|\cos \theta_{\nu}\right|\right),
$$

where we approximate the base diameter as $D_{b}=a+b$, and where we already account for all four modules. Conservatively, we have also computed the number of $\mu^{+}$-like events restricting ourselves to the contained topology. This assumption eases the comparison with respect to the case in which no flavor tagging is available and ensures good energy reconstruction for the full muon like event sample. 



Figure 5.6: Relative neutrino energy resolution $\sigma_{E} / E_{\nu}$ (left) and absolute neutrino angular resolution $\sigma_{\theta}$ (right) as a function of neutrino energy $E_{\nu}$ assumed in this study, for $\nu_{e}, \bar{\nu}_{e}, \nu_{\mu}$ and $\bar{\nu}_{\mu}$ charged-current interactions on argon nuclei. Figure taken from Ref. [10].

\begin{tabular}{|c|c|c|c|c|}
\hline Parameter & $\nu_{e}$ & $\bar{\nu}_{e}$ & $\nu_{\mu}$ & $\bar{\nu}_{\mu}$ \\
\hline $\mathrm{A}$ & 22.4 & 20.8 & 22.0 & 20.3 \\
$\mathrm{~B}$ & 0.582 & 0.680 & 0.548 & 0.625 \\
\hline
\end{tabular}

Table 5.1: Numerical values for the parameters appearing in Eq. (5.15), and defining the energy dependence of the neutrino energy resolution assumed in this work.

As for the energy and angular smearing inherent to reconstruction processes and final state hadronic interactions within Ar-nuclei, $R_{e}^{\mu}\left(E_{r, \nu}, E_{\nu}\right)$ and $R_{\theta}^{\mu}\left(\theta_{r, \nu}, \theta_{\nu}\right)$ in Eq. (5.10), and $R_{e}^{e}\left(E_{r, \nu}, E_{\nu}\right)$ and $R_{\theta}^{e}\left(\theta_{r, \nu}, \theta_{\nu}\right)$ in Eq. (5.13), are taken to be Gaussian functions. The assumed Gaussian widths $\sigma_{E} / E$ and $\sigma_{\theta}$ for $\nu_{e}, \bar{\nu}_{e}, \nu_{\mu}$ and $\bar{\nu}_{\mu}$ charged-current interactions on argon are shown in Fig. 5.6. We use the dashed curves in the figure to parameterize the resolutions as functions of the neutrino energy $E_{\nu}$, according to:

$$
\begin{gathered}
\sigma_{E} / E_{\nu}=A / E_{\nu}^{B}, \\
\sigma_{\theta}=C / E_{\nu}+D .
\end{gathered}
$$

The $A$ and $B$ parameters describing the relative neutrino energy resolution (in percent), and the $C$ and $D$ parameters describing the neutrino angle resolution (in degrees), are given in Tabs. 5.1 and 5.2, respectively. The parameters are given separately for each neutrino flavor: $\nu_{e}, \bar{\nu}_{e}, \nu_{\mu}$ and $\bar{\nu}_{\mu}$. The parameters in Eq. (5.15) assume that $E_{\nu}$ is expressed in $\mathrm{GeV}$. 


\begin{tabular}{|c|c|c|c|c|}
\hline Parameter & $\nu_{e}$ & $\bar{\nu}_{e}$ & $\nu_{\mu}$ & $\bar{\nu}_{\mu}$ \\
\hline $\mathrm{C}$ & 7.85 & 8.42 & 7.79 & 8.46 \\
$\mathrm{D}$ & 3.70 & 2.31 & 3.71 & 2.29 \\
\hline
\end{tabular}

Table 5.2: Numerical values for the parameters appearing in Eq. (5.15), and defining the energy dependence of the neutrino angle resolution assumed in this work.

These resolution functions were obtained via fast Monte-Carlo simulations as follows, similarly to what was done in 140,200 . First, large samples of monoenergetic neutrino-argon interactions are simulated with GENIE, for the various neutrino flavors $\left(\nu_{e}, \bar{\nu}_{e}, \nu_{\mu}\right.$ and $\left.\bar{\nu}_{\mu}\right)$ and for the relevant neutrino energy range 0.5-8 GeV. The GENIE simulation includes nuclear effects. Second, for each event, each final state particle exiting the nucleus has its kinetic energy and angular direction smeared according to the assumptions described in [200]. The relative energy resolutions are taken to be $1 \% / \sqrt{E_{e}}+1 \%, 3 \%$ and $30 \% / \sqrt{E_{\text {had }}}$ for electrons, muons and hadrons, respectively, where $E_{e}$ and $E_{\text {had }}$ are expressed in $\mathrm{GeV}$. The absolute angular resolutions are taken to be $1^{\circ}, 1^{\circ}$ and $10^{\circ}$ for the same three final state particle categories and for all energies. Third, the incoming neutrino energy and direction of each interaction is reconstructed as follows:

$$
\begin{gathered}
E_{r, \nu}=K_{r, l}+m_{l}+\sum_{h} K_{r, h}, \\
\theta_{r, \nu}=\arccos \left(p_{z r, \nu} /\left|\vec{p}_{r, \nu}\right|\right),
\end{gathered}
$$

where $K_{r, l}$ and $K_{r, h}$ are the reconstructed charged lepton and hadron kinetic energies, $m_{l}$ is the charged lepton mass, the sum $\sum_{h}$ is intended over all final state hadrons, and $\vec{p}_{r, \nu} \equiv \vec{p}_{r, l}+\sum_{h} \vec{p}_{r, h}$ is the reconstructed 3-momentum of the incoming neutrino, where the true neutrino direction is defined along the $\mathrm{z}$ axis. Fourth, histograms of the reconstructed neutrino energy and direction are obtained for each (neutrino flavor, neutrino energy) simulated data sample. Fifth, $\sigma_{E} / E_{\nu}$ and $\sigma_{\theta}$ for each data sample are obtained from a Gaussian fit to the energy histogram and from the mean of the angle histogram, respectively. The resolution functions are shown in Fig. 5.6 for each sample via marker symbols. Sixth, the energy dependence of the resolutions functions is parameterized according to Eq. 5.15). The behavior of these functions can be easily understood. The main effect is that both $\sigma_{E} / E$ and $\sigma_{\theta}$ improve noticeably as the neutrino energy increases. For $\sigma_{E} / E$, this is a direct consequence of the relative energy resolutions assumed for electrons and (especially) hadrons, improving as the particle energies increase. 
For $\sigma_{\theta}$, this is due to the Fermi momentum of the target nucleon, whose angular smearing effect is more important at low neutrino energies. A second, smaller, effect can also be appreciated in Fig. 5.6, namely that antineutrino resolutions are slightly better than neutrino ones, both for $\sigma_{E} / E$ and $\sigma_{\theta}$. On the one hand, this is due to the fact that the average inelasticity (or energy fraction carried away by final state hadrons) is somewhat lower in antineutrino interactions [217, and on the other because hadron resolutions are substantially worse than charged lepton ones. An even smaller difference can be appreciated between the relative energy resolutions of electron and muon antineutrinos of the same energy. In this case, electron antineutrino energy resolutions are slightly better, because of the better assumed accuracy in reconstructing electron energy $\left(1 \% / \sqrt{E_{e}}+1 \%\right)$ compared to muon energy (3\%).

Our energy resolution assumptions in Fig. 5.6 are similar to the ones in 140 , 200], that use similar methodologies and assumptions. They are qualitatively similar also to the ones obtained in more recent studies, see Refs. 147,217. On the other hand, we are not aware of other neutrino angular resolutions studies in LArTPCs to compare our findings with.

\subsubsection{Analyses and Results}

Here, we describe the statistical analysis and how we extract the sensitivity to the neutrino mass ordering. In order to emphasize the impact of the muon capture in argon, we present two possible analyses. The first case will assume that no charge identification is possible. Then, we will focus on the extra bonus that the muon capture in argon process provides.

In the following, we define a fiducial mass ordering, true ordering (TO), in order to generate mock data. Then, we try to reconstruct the event rates using the wrong ordering (WO) assumption. Although there is some preference for normal neutrino mass ordering, as previously stated, we shall study also the case of inverted ordering as TO.

We use Eqs. $((5.10))$ and $((5.13))$ to generate our mock data, using the oscillation parameters from Tab. 5.3 and assuming a $400 \mathrm{kt} \cdot \mathrm{yr}$ exposure. We will present our results as a function of the atmospheric angle $\theta_{23}$. Therefore, there is no fixed value for this angle in the table. Notice that, since our main sensitivity comes from the $\nu_{\mu} \rightarrow \nu_{\mu}$ channel, the effects of the $\mathrm{CP}$ violating phase $\delta$ are negligible and therefore we set $\delta=0$, finding very similar results for other values of the CP phase.

Next, we try to reconstruct the event rates following the two methods mentioned above. Before presenting our results, let us discuss our treatment of sys- 


\begin{tabular}{|c|c|c|}
\hline parameter & Normal ordering & Inverted Ordering \\
\hline$\Delta m_{21}^{2}$ & $7.55 \times 10^{-5} \mathrm{eV}^{2}$ & $7.55 \times 10^{-5} \mathrm{eV}^{2}$ \\
$\Delta m_{31}^{2}$ & $2.50 \times 10^{-3} \mathrm{eV}^{2}$ & $-2.42 \times 10^{-3} \mathrm{eV}^{2}$ \\
$\sin ^{2} \theta_{12}$ & 0.320 & 0.320 \\
$\sin ^{2} \theta_{13}$ & 0.02160 & 0.0222 \\
$\delta$ & 0 & 0 \\
\hline
\end{tabular}

Table 5.3: The oscillation parameters used to generate the mock data 2]. We use various values for the atmospheric angle $\theta_{23}$.

tematic uncertainties. We consider several sources of systematic uncertainties in our analyses, coming from the fact that we do not have a perfect knowledge of the atmospheric flux and detector response. In particular, we include an overall rate normalization error accounting for both flux normalization and detector efficiency uncertainties, an error on the $\nu / \bar{\nu}$ atmospheric flux ratio, and an error on the $\nu_{\mu} / \nu_{e}$ atmospheric flux ratio. We follow Ref. [200] and assume a 15\%, 5\%, and $2 \%$ Gaussian error on these three quantities, respectively. We have verified that adding a systematic on the spectral index of the neutrino flux would have a negligible effect. As explained in the previous section, we also add an uncertainty on $\epsilon^{\text {cap }}$, see Eq. (5.9). Apart from the systematic uncertainties, we also marginalize over the oscillation parameters $\Delta m_{31}^{2}, \sin ^{2} \theta_{13}$ and $\sin ^{2} \theta_{23}$ within their current $3 \sigma$ ranges for both orderings, namely $\left|\Delta m_{31}^{2}\right| \in[2.31,2.60] \times 10^{-3}$ $\mathrm{eV}^{2}, \sin ^{2} \theta_{13} \in[0.0196,0.0244]$ and $\sin ^{2} \theta_{23} \in[0.455,0.599]$. It is well known that the solar parameters do not have big effects in atmospheric neutrino oscillations, hence, they are fixed to their best-fit values throughout the analysis.

\section{Method A: Analysis without muon capture tagging}

In this case, muons and antimuons cannot be distinguished. We therefore build a $\chi^{2}$ function in the following way:

$$
\chi_{A}^{2}\left(\sin ^{2} \theta_{23}^{\text {true }}\right)=\min _{\text {sys }, \Delta m_{31}^{2}, \theta_{13}, \theta_{23}}\left\{\chi_{\mu^{-}+\mu^{+}}^{2}+\chi_{e^{-}+e^{+}}^{2}\right\} .
$$

We use a Poissonian $\chi^{2}$, which for muons is

$$
\chi_{\mu^{-}+\mu^{+}}^{2}=2 \sum_{i, j} N_{i, j, \mu}^{\mathrm{WO}}-N_{i, j, \mu}^{\mathrm{TO}}+N_{i, j, \mu}^{\mathrm{TO}} \log \left(\frac{N_{i, j, \mu}^{\mathrm{TO}}}{N_{i, j, \mu}^{\mathrm{WO}}}\right),
$$


where $N_{i, j, \mu}^{\mathrm{TO}(\mathrm{WO})}=N_{i, j, \mu^{+}}^{\mathrm{TO}(\mathrm{WO})}+N_{i, j, \mu^{-}}^{\mathrm{TO}(\mathrm{WO})}$ is the sum of the muon and antimuon contributions. The same formula applies to $\chi_{e^{-}+e^{+}}^{2}$, with the replacement $\mu \rightarrow e$. The results of our analysis with method A are shown as red curves in Fig. 5.7. Note that the sensitivity ranges between 1.5 and $3.5 \sigma$ approximately when normal ordering is the TO (solid lines) and between 1.5 and $2 \sigma$ for true inverted ordering (dashed line).

\section{Method B: Analysis with muon capture tagging}

In this other strategy, we use muon capture to distinguish $\sim 72 \%$ of the muons from antimuons. Therefore, this time our $\chi^{2}$ function contains three terms, namely

$$
\chi_{B}^{2}\left(\sin ^{2} \theta_{23}^{\text {true }}\right)=\min _{\text {sys }, \Delta m_{31}^{2}, \theta_{13}, \theta_{23}}\left\{\chi_{\mu}^{2, \text { cap }}+\chi_{\mu}^{2, \text { rest }}+\chi_{e^{-}+e^{+}}^{2}\right\} .
$$

The electron term is the same as for method A, while the other two terms, corresponding to the events with muon capture (cap) and all other events (rest), are given by

$$
\chi_{\mu}^{2, X}=2 \sum_{i, j} N_{i, j, \mu}^{\mathrm{WO}, X}-N_{i, j, \mu}^{\mathrm{TO}, X}+N_{i, j, \mu}^{\mathrm{TO}, X} \log \left(\frac{N_{i, j, \mu}^{\mathrm{TO}, X}}{N_{i, j, \mu}^{\mathrm{WO}, X}}\right),
$$

where $X \in\{$ cap, rest $\}$, see Eqs. (5.11) and 5.12) . The results of the analysis with muon capture are shown in Fig. 5.7 by the blue curves. As before, true normal ordering is shown as a solid line, while the case of true inverted ordering is represented by a dashed line. The grey band in the figure represents the current $1 \sigma$ allowed region for $\sin ^{2} \theta_{23}$. Note how the sensitivity to the mass ordering is now at the 2.5-4 $\sigma$ level, implying an important improvement with respect to the results obtained with method A. In particular, for the current best-fit point [2] we find that, using atmospheric neutrinos with muon capture, DUNE could measure the neutrino mass ordering at the $3.5 \sigma$ level. Our method B results can also be compared with the results in the DUNE Conceptual Design Report [140], where a similar sensitivity reach and dependence on $\sin ^{2} \theta_{23}$ were obtained. Compared to [140], however, our results more clearly highlight the importance of the muon capture tag. 


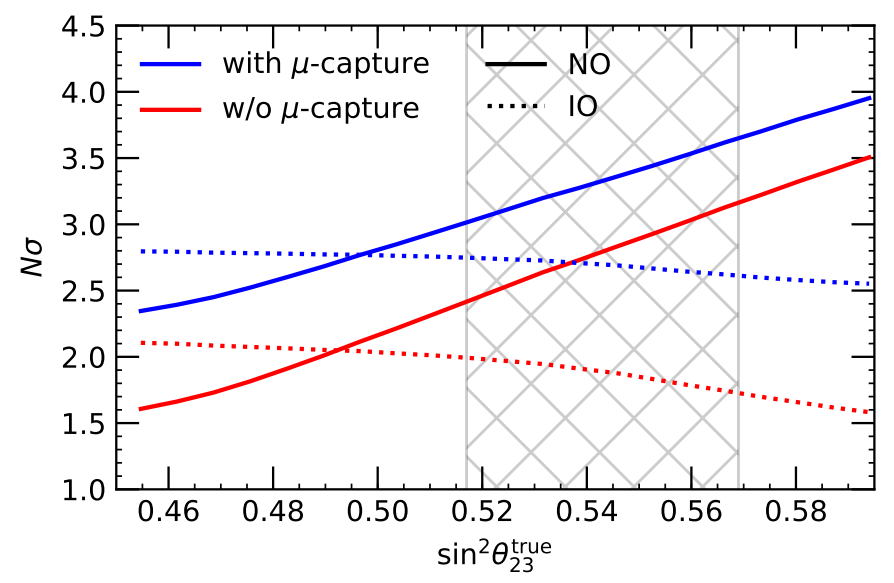

Figure 5.7: The DUNE sensitivity to the neutrino mass ordering as a function of $\sin ^{2} \theta_{23}^{\text {true }}$. Red (blue) lines correspond to the analysis method A (B). Solid lines are for normal ordering as true ordering, while dashed lines show the sensitivity in the case of true inverted ordering. The grey band corresponds to the current $1 \sigma$ region for the atmospheric angle. Figure taken from Ref. $[10]$. 



\section{Chapter 6}

\section{CPT violation and DUNE}

In this chapter, we study the sensitivity of the DUNE experiment to the scenario introduced in chapter 3 . There, we presented the current bounds on CPT violation in the neutrino sector. Here, we will see how much DUNE can improve these bounds. In addition, we consider one certain benchmark point as a case study for the estimation of the DUNE potential to detect CPT violation and distinguish it from other new physics scenarios. The results of this chapter are published in Refs. [5] and [13].

\subsection{Improving the current bounds on CPT violation at DUNE}

\subsubsection{Simulation of the DUNE experiment}

The simulation of DUNE follows the description given in chapter 5. The only difference is that, this time, we study the neutrino and antineutrino modes separately, where as in chapter 3 neutrinos oscillate with parameters $\theta_{12}, \theta_{13}, \theta_{23}$, $\Delta m_{21}^{2}, \Delta m_{31}^{2}, \delta$ and antineutrinos with $\bar{\theta}_{12}, \bar{\theta}_{13}, \bar{\theta}_{23}, \Delta \bar{m}_{21}^{2}, \Delta \bar{m}_{31}^{2}, \bar{\delta}$. Unfortunately, using GLoBES has one disadvantage in the treatment of backgrounds, since for a given channel, for instance the neutrino channel, the antineutrino backgrounds are oscillated with the same probability as the neutrino signals and vice versa. While it is possible to use a customized probability engine in GLoBES, we have actually checked that the effect of the backgrounds is negligible. Therefore, in our analysis we oscillate the backgrounds with the same parameters as the signal. In any case, in order to mitigate the impact such a simplification can have, we have increased the systematic errors due to misidentification of neutrinos by 


\begin{tabular}{lc}
\hline parameter & value \\
\hline$\Delta m_{21}^{2}$ & $7.56 \times 10^{-5} \mathrm{eV}^{2}$ \\
$\Delta m_{31}^{2}$ & $2.55 \times 10^{-3} \mathrm{eV}^{2}$ \\
$\sin ^{2} \theta_{12}$ & 0.321 \\
$\sin ^{2} \theta_{23}$ & $0.43,0.50,0.60$ \\
$\sin ^{2} \theta_{13}$ & 0.02155 \\
$\delta$ & $1.50 \pi$ \\
\hline
\end{tabular}

Table 6.1: Oscillation parameters used to simulate neutrino and antineutrino data analyzed in Sec. 6.1 .2

antineutrinos and vice versa by a further $25 \%$ over the original error given by the collaboration 1

\subsubsection{DUNE sensitivity to CPT-violating neutrino oscillation parameters}

In this section, we study the sensitivity of DUNE to measure CPT violation in the neutrino and antineutrino oscillation parameters. For a given oscillation parameter $x$, we first perform simulations of the DUNE experiment with $\Delta x=$ $|x-\bar{x}|=0$, i.e., assuming equal parameters for neutrinos and antineutrinos. Next, we estimate the sensitivity of DUNE to $\Delta x \neq 0$. In our analysis of the DUNE neutrino and antineutrino mode, we vary freely all the oscillation parameters except the solar ones, since their effect is negligible in DUNE. The treatment of the reactor angle $\bar{\theta}_{13}$ in the case of the antineutrino mode is also slightly different, since we put a prior on $\sin ^{2} \bar{\theta}_{13}=0.02155 \pm 0.0009086$ motivated by the current knowledge due to the reactor experiments which use only antineutrinos. To simulate the data in DUNE, we consider as true parameters the values in Table 6.1. To explore possible correlations between DUNE CPT sensitivity and the atmospheric octant, we have chosen three values for $\theta_{23}$. First, we choose one true value in the lower octant. Then, we also consider $\theta_{23}$ in the upper octant as well as maximal atmospheric mixing. After minimizing over all parameters except $x$ and $\bar{x}$, we calculate

$$
\chi^{2}(\Delta x)=\chi^{2}(|x-\bar{x}|)=\min _{\substack{x, \bar{x} \\|x-\bar{x}|=\Delta x}}\left\{\chi^{2}(x)+\chi^{2}(\bar{x})\right\}
$$

\footnotetext{
${ }^{1}$ Note that using the technique of Chap. 5 the muon neutrino background in antineutrino mode could be importantly reduced.
} 
where we have considered all possible combinations of $|x-\bar{x}|$. Note that this last equation is a simplification, since actually also the neutrino (antineutrino) sample depends on antineutrino (neutrino) parameters because there is always a socalled wrong-sign contamination present. Also, other backgrounds might contain both neutrinos and antineutrinos in both modes. Therefore, one could actually use a more general $\chi_{\nu / \bar{\nu}}^{2}(x, \bar{x})$ to compute the total $\chi^{2}$ function, considering the appropriate neutrino or antineutrino parameters for the signal and also for the backgrounds 2 However, as explained in the last subsection, we have checked that the effect of the backgrounds is very small and in our simplified treatment can be counted in for by simply increasing the systematic uncertainties. Our results are presented in Fig. 6.1, where we plot three different lines, labeled as "high", "max" and "low". These refer to the assumed value for the atmospheric angle: in the lower octant (low), maximal mixing $(\max )$ or in the upper octant (high). There, one can see that there is no sensitivity to $\Delta\left(\sin ^{2} \theta_{13}\right)=\left|\sin ^{2} \theta_{13}-\sin ^{2} \bar{\theta}_{13}\right|$, nor to $\Delta \delta=|\delta-\bar{\delta}|$. Note that, in the case of $\Delta\left(\sin ^{2} \theta_{13}\right)$, there would be a $3 \sigma$ exclusion only for $\Delta\left(\sin ^{2} \theta_{13}\right) \approx 0.015$, which is basically of the order of $\sin ^{2} \bar{\theta}_{13}=0.02155$. For $\Delta \delta$ we would not disfavor any value at more than $2 \sigma$ confidence level.

On the contrary, we obtain very interesting results for $\Delta\left(\Delta m_{31}^{2}\right)$ and $\Delta\left(\sin ^{2} \theta_{23}\right)$. First of all, we find that DUNE should be able to set bounds on $\Delta\left(\Delta m_{31}^{2}\right)$ tighter than $8.1 \times 10^{-5}$ at $3 \sigma$ confidence level. This would imply an improvement of one order of magnitude with respect to the old bound in Ref. [132 and four orders of magnitude with respect to the neutral Kaon bound, once it is viewed as a bound on the mass squared. Concerning the atmospheric mixing angle, we obtain different results depending on the true value assumed to simulate DUNE data. In the lower right panel of Fig. 6.1 we see the different behavior obtained for maximal $\theta_{23}$ and $\theta_{23}$ in the upper or lower octant. In the case of true maximal mixing, the sensitivity increases with $\Delta\left(\sin ^{2} \theta_{23}\right)$, as one might expect. However, if we assume the true values to be in the first or second octant, a degenerate solution appears in the complementary octant, as can be seen in Fig. 6.2. Since there is no prior on $\sin ^{2} \theta_{13}$ in the neutrino mode, the second fake solution survives with $\Delta \chi^{2} \approx 0.15$. Hence, in minimizing over $\left|\sin ^{2} \theta_{23}-\sin ^{2} \bar{\theta}_{23}\right|$, a second minimum appears if one value is in the lower octant and the other one in the upper one close to the degenerate solution. This means that, if in nature, for example, $\sin ^{2} \theta_{23} \approx 0.43$ and $\sin ^{2} \bar{\theta}_{23} \approx 0.60$, DUNE would be blind to this difference, as long as no better determination of $\theta_{13}$ is obtained. This behavior can be understood by looking at the $\Delta \chi^{2}$ profiles of the atmospheric angles in Fig. 6.2. Note that the neutrino channel alone is basically blind to the octant

\footnotetext{
${ }^{2}$ This technique was used in Ref. [218 to analyze atmospheric neutrino data at Super-K.
} 

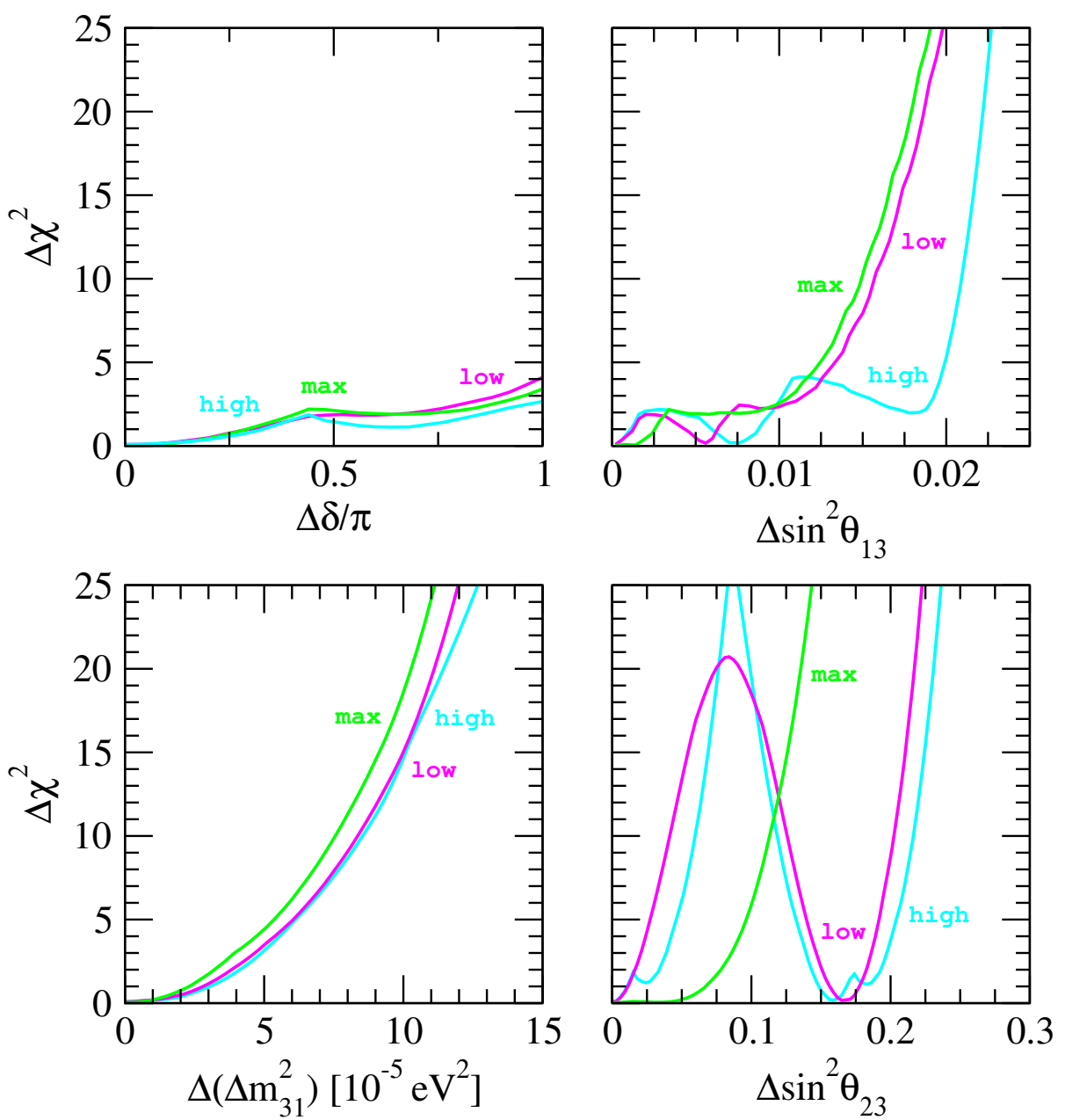

Figure 6.1: The sensitivities of DUNE to the difference of neutrino and antineutrino parameters: $\Delta \delta, \Delta\left(\Delta m_{31}^{2}\right), \Delta\left(\sin ^{2} \theta_{13}\right)$ and $\Delta\left(\sin ^{2} \theta_{23}\right)$ for the atmospheric angle in the lower octant (magenta line), in the upper octant (cyan line) and for maximal mixing (green line). Figure taken from Ref. [5].

discrimination and, then, the degenerate solution always appears. Even in the antineutrino channel, the degeneration disappears only if $\sin ^{2} \bar{\theta}_{23}$ lies in the lower octant. If it lies in the upper octant, the degenerate solution also shows up. This is because the constraint on $\sin ^{2} \bar{\theta}_{13}$ from Daya Bay pulls $\sin ^{2} \bar{\theta}_{23}$ into the lower octant. Hence, both solutions appear also in this case. 



Figure 6.2: The sensitivity to the atmospheric angle for true values in the lower (left) and upper (right) octant. Figure taken from Ref. [5].

\subsubsection{Obtaining imposter solutions}

In neutrino experiments whose beam is produced at accelerators, neutrino and antineutrino data are obtained on separated runs. However, courtesy of the smallness of the antineutrino cross section as compared to the neutrino one, roughly only one third of the data are obtained with the former, implying larger statistical errors. Because of that, and under the seemingly "light" assumption of CPT conservation, it is tempting to perform a joint analysis. Such a path, as we have shown so far, is not risk-free. First of all, the opportunity to set the best limit on the possible CPT violation in the mass-squared of elementary particles and antiparticles is lost. And most important, if CPT is violated in Nature, the gain in statistics is done by sacrificing the physics. The outcome of the joint data analysis will not be that of either channel but what we call an imposter solution. A solution which results from the combined analysis but does not correspond to the true solution of either channel.

Nevertheless, in experiments and also global fits one normally assumes CPT to be conserved. In this case, the $\chi^{2}$-functions are computed according to

$$
\chi_{\text {total }}^{2}=\chi^{2}(\nu+\bar{\nu}) .
$$




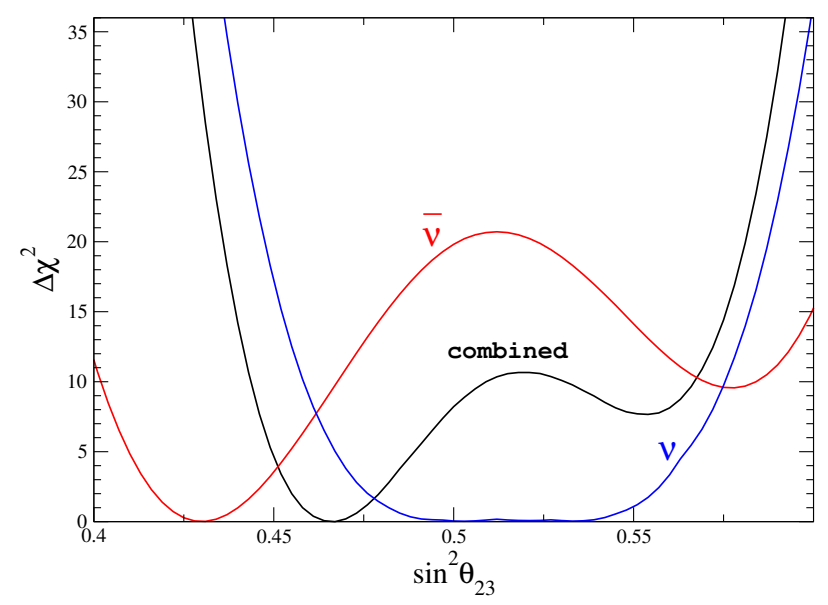

Figure 6.3: DUNE sensitivity to the atmospheric angle for neutrinos (blue), antineutrinos (red) and to the combination of both under the assumption of CPT conservation (black). Figure taken from Ref. [5].

Here, the contributions from neutrino and antineutrino channels are added, before marginalizing over any of the parameters to obtain $\chi^{2}$ profiles. In contrast, in Eq. (6.1) we first analyzed data in neutrino and antineutrino mode separately, obtaining $\chi^{2}$ profiles for neutrino and antineutrino oscillation parameters and then created the profiles for $\Delta x$. For this reason, in the CPT conserving case, we do not encounter the possible problems in the treatment of the background mentioned in Sec. 6.1.1.

In this section, we assume that CPT is violated when creating the fake data, but treat our results as if it was conserved in the statistical analysis. We assume that the true value for atmospheric neutrino mixing is $\sin ^{2} \theta_{23}=0.5$, while the antineutrino mixing angle is given by $\sin ^{2} \bar{\theta}_{23}=0.43$. The remaining oscillation parameters are fixed to the values in Tab. 6.1. If we now combine the results of our simulations for these values, but assume the same mixing for neutrinos and antineutrinos in the reconstruction analysis, we obtain the sensitivity to the atmospheric angle presented in Fig. 6.3. We also plot the individual reconstructed profiles for neutrinos and antineutrinos for comparison. By combining the two results, we obtain the best-fit value at $\sin ^{2} \theta_{23}^{\text {comb }}=0.467$, disfavoring the true values at close to $3 \sigma$ and more than $5 \sigma$ for neutrino and antineutrino parameters, respectively.

We also performed a similar study fixing $\sin ^{2} \theta_{23}=\sin ^{2} \bar{\theta}_{23}=0.430$, but choosing $\delta=0(0.5 \pi)$ and $\bar{\delta}=0.5 \pi(0)$. The results are presented in the left 

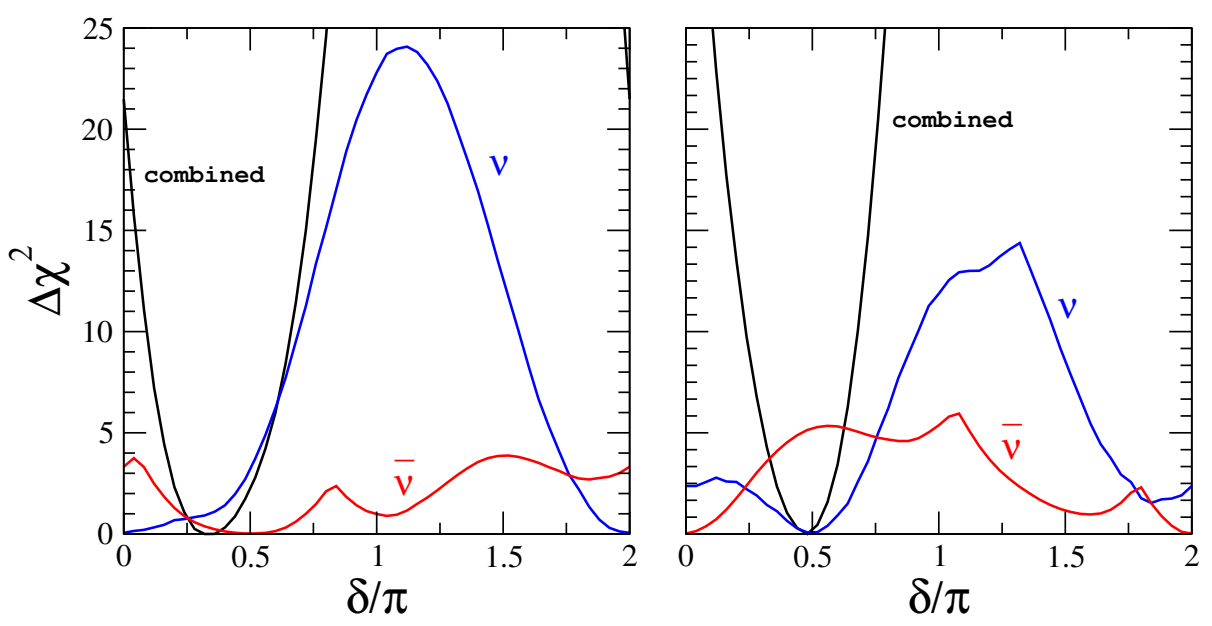

Figure 6.4: Obtaining imposter solutions for the CP-phases. As before, blue (red) line corresponds to DUNE sensitivity for neutrinos (antineutrinos). Black line corresponds to the combined neutrino + antineutrino sensitivity if CPT conservation is assumed. We assume the true values to be $\delta=0(0.5 \pi)$ and $\bar{\delta}=0.5 \pi(0)$ in the left (right) panel. Figure taken from Ref. 5 .

(right) panel of Fig. 6.4. On combining both channels the value $\delta=0$ gets highly disfavored, close to $5 \sigma$ in one case and more than $7 \sigma$ in the other, even though it is one of the true values chosen to simulate neutrino oscillations.

\begin{tabular}{lc}
\hline parameter & value \\
\hline$\Delta m_{31}^{2}$ & $2.60 \times 10^{-3} \mathrm{eV}^{2}$ \\
$\Delta \bar{m}_{31}^{2}$ & $2.62 \times 10^{-3} \mathrm{eV}^{2}$ \\
$\sin ^{2} \theta_{23}$ & 0.51 \\
$\sin ^{2} \bar{\theta}_{23}$ & 0.42 \\
\hline \multicolumn{1}{c}{$m_{21}^{2}, \Delta \bar{m}_{21}^{2}$} & $7.56 \times 10^{-5} \mathrm{eV}^{2}$ \\
$\sin ^{2} \theta_{12}, \sin ^{2} \bar{\theta}_{12}$ & 0.321 \\
$\sin ^{2} \theta_{13}, \sin ^{2} \bar{\theta}_{13}$ & 0.02155 \\
$\delta, \bar{\delta}$ & $1.50 \pi$ \\
\hline
\end{tabular}

Table 6.2: Oscillation parameters considered in the analysis of Sec. 6.1.4. 


\subsubsection{Probing the $\mathrm{T} 2 \mathrm{~K}$ neutrino and antineutrino analysis in DUNE}

In this section, we explore the sensitivity of DUNE to the separate analysis of neutrino and antineutrino data performed by the T2K Collaboration in Ref. [103. Therefore, we consider the best fit values of this analysis as the true values for the atmospheric parameters: $\Delta m_{31}^{2}=2.60 \times 10^{-3} \mathrm{eV}^{2}$ and $\sin ^{2} \theta_{23}=0.51$ for neutrinos and $\Delta \bar{m}_{31}^{2}=2.62 \times 10^{-3} \mathrm{eV}^{2}$ and $\sin ^{2} \bar{\theta}_{23}=0.42$ for antineutrinos. The analysis considers only normal mass ordering, as we assume that the current hint on this being the path followed by Nature will be solid when DUNE turns on. The oscillation parameters used to create the fake data of this section are summarized in Table 6.2 .

We, then, follow the same procedure as before and try to reconstruct the fake data within the sensitivity of the DUNE experiment. As before, in the antineutrino channel, a prior on the determination of the reactor mixing angle, $\sin ^{2} \bar{\theta}_{13}=0.02155 \pm 0.00090$ 86, is considered. We present the results of the analysis of neutrino and antineutrino data together in the same plot, projecting over two-dimensional regions and marginalizing over the other parameters not plotted. In Fig. 6.5 (left) we present the allowed regions at $2 \sigma, 3 \sigma$ and $4 \sigma$ in the atmospheric plane $\left(\sin ^{2} \theta_{23}, \Delta m_{31}^{2}\right)$. There, one can see that, unlike what happens for T2K, in DUNE there would be no overlap of the allowed regions at the $3 \sigma$ level, although there would be still some overlap at the $4 \sigma$ level. To make this point even clearer, we have plotted in Fig. 6.7 the sensitivity to $\Delta\left(\Delta m_{31}^{2}\right)=\left|\Delta m_{31}^{2}-\Delta \bar{m}_{31}^{2}\right|$ and $\Delta \sin ^{2} \theta_{23}=\left|\sin ^{2} \theta_{23}-\sin ^{2} \bar{\theta}_{23}\right|$ from this analysis. There, one can see that, for the mass splittings, CPT conservation remains allowed at $1 \sigma$ while, for the mixing angles, the hypothesis of CPT conservation is disfavored at around the $5 \sigma$ level.

Note also that the antineutrino run in DUNE alone could resolve the octant of the atmospheric mixing angle at $3 \sigma$ if $\sin ^{2} \bar{\theta}_{23}=0.42$ turns out to be the true value. In the right panel of Fig. 6.5 we see that the DUNE neutrino mode is not very sensitive to the reactor angle $\theta_{13}$, since values as large as $\sin ^{2} \theta_{13}=0.034$ (far from the Daya Bay upper bound) are allowed at the $2 \sigma$ level. On the other hand, in the right panel of Fig. 6.6 one can see the impact of considering a prior on $\sin ^{2} \bar{\theta}_{13}$ on the determination of $\bar{\delta}$. The lack of a prior in the neutrino mode results in a reduced sensitivity to the CP phase $\delta$. This can also be observed in Fig. 6.8, where we plot the $\Delta \chi^{2}$ profiles of the oscillation parameters.

As commented above, the main constraint on the reactor angle in antineutrino oscillations comes from the Daya Bay experiment [86], while in the case of neutrino oscillations no such measurement exists. So, even though the neutrino 

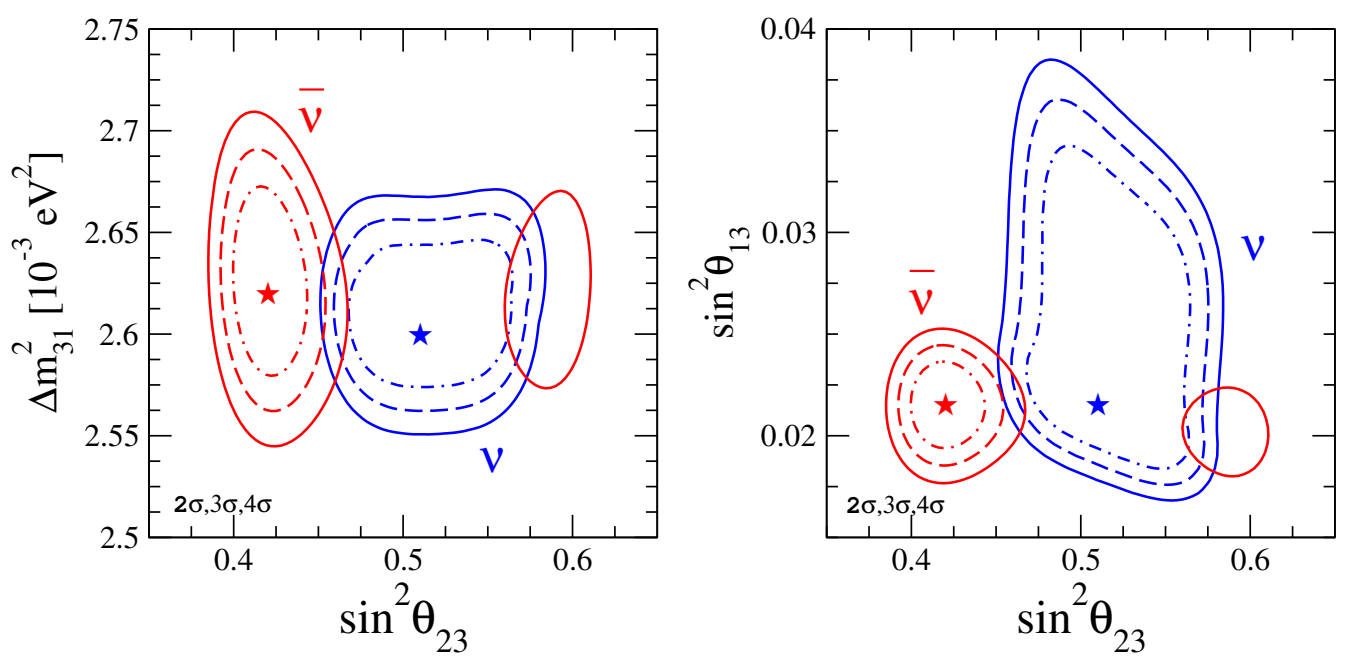

Figure 6.5: DUNE expected regions at $2 \sigma, 3 \sigma$ and $4 \sigma$ in the atmospheric plane $\sin ^{2} \theta_{23}-\Delta m_{31}^{2}$ (left) and the $\sin ^{2} \theta_{23}-\sin ^{2} \theta_{13}$ plane (right) for neutrinos (blue) and antineutrinos (red). The stars indicate the assumed true values for neutrino (blue) and antineutrino (red) oscillation parameters. Figure taken from Ref. [5].

channel has higher statistics because of the reduced cross section for antineutrinos, the constraints from Daya Bay improve drastically the sensitivity in the antineutrino channel.

\subsection{Robustness of the CPT results: CPT versus NSI}

Given the impact an observation of CPT violation would have, it is crucial to distinguish a true, genuine CPT violation from just a new, unknown interaction, no matter how sophisticated.

In this section, we confront the presence of intrinsic CPT violation with new neutrino interactions with matter, usually known as neutrino non-standard interactions (NSI) ${ }^{3}$. NSI are an agnostic way to parameterize all the CPT preserving possibilities economically and, therefore, provide the ideal tool to discriminate between a legitimate CPT violation, a phenomenon that challenges our description of Nature in terms of local relativistic quantum fields, and a more or less

\footnotetext{
${ }^{3}$ Note that CPT violation might also be induced through different new physics scenarios, such as decoherence $219 \mid 221$, or Lorentz violation $14|222| 224$.
} 


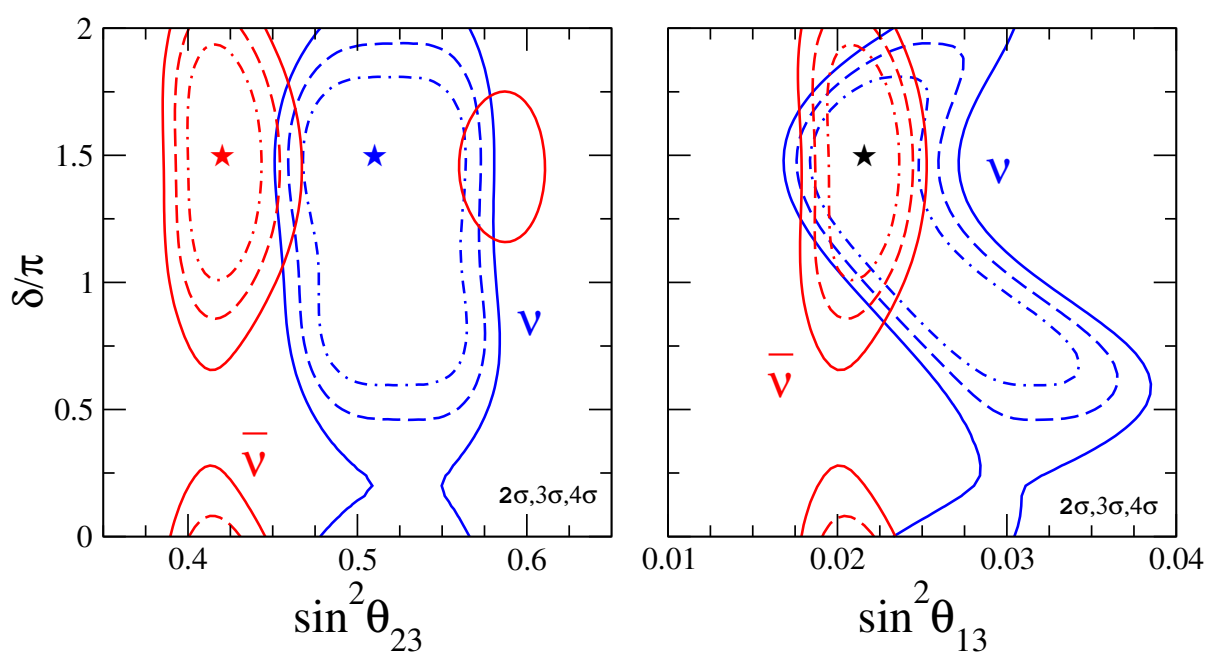

Figure 6.6: DUNE expected regions at $2 \sigma, 3 \sigma$ and $4 \sigma$ in the $\sin ^{2} \theta_{23}-\delta$ plane (left) and the $\sin ^{2} \theta_{13}-\delta$ plane (right) for neutrinos (blue) and antineutrinos (red). The stars indicate the assumed true values for neutrino (blue) and antineutrino (red) oscillation parameters and for both (black) in the right panel. Figure taken from Ref. [5].
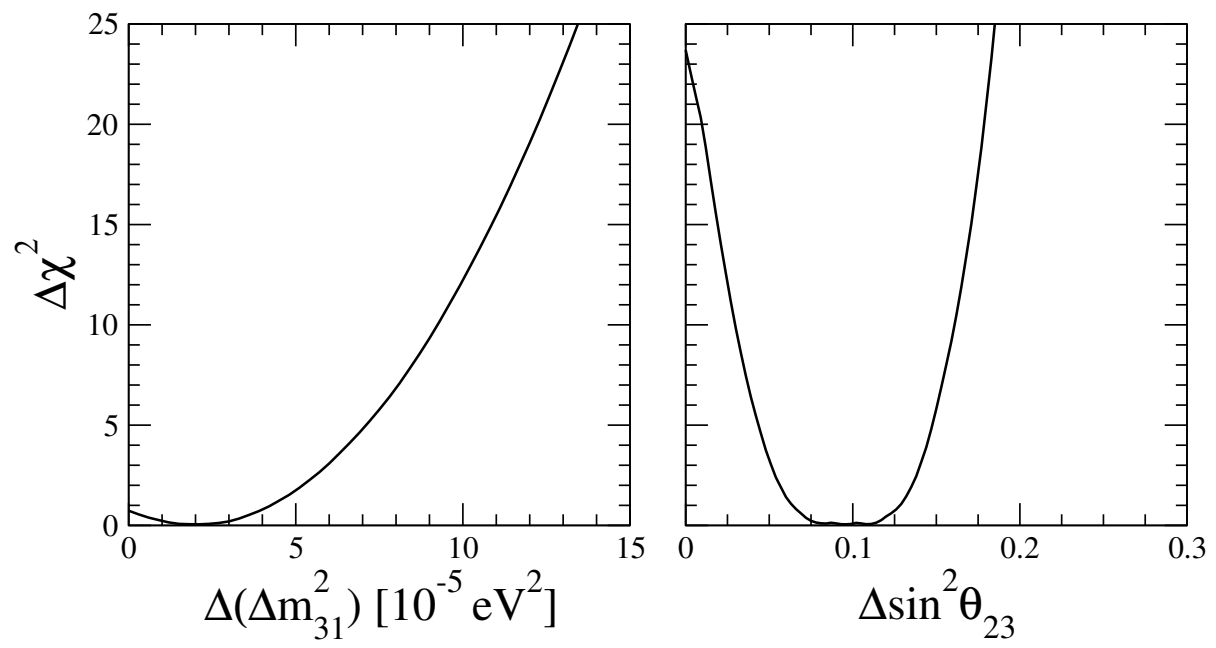

Figure 6.7: $\Delta \chi^{2}$ profiles as a function of $\Delta\left(\Delta m_{31}^{2}\right)$ and $\Delta \sin ^{2} \theta_{23}$ assuming the parameters obtained by T2K as true values. Figure taken from Ref. [5]. 

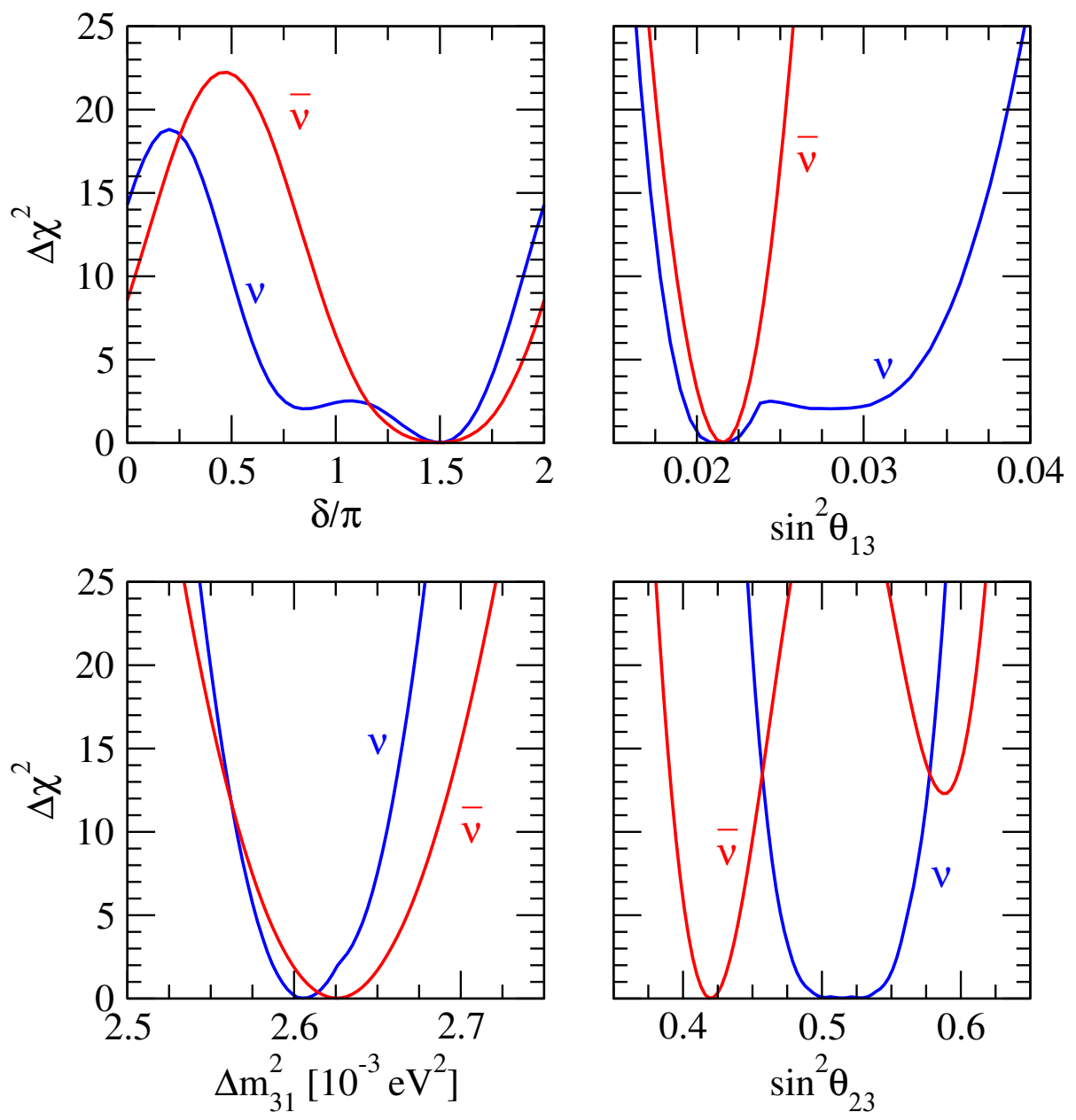

Figure 6.8: $\Delta \chi^{2}$ profiles as a function of $\delta, \sin ^{2} \theta_{13}, \Delta m_{31}^{2}$ and $\sin ^{2} \theta_{23}$ from the separate analysis of neutrino and antineutrino data in DUNE assuming the atmospheric parameters as determined by the separate analysis of the $\mathrm{T} 2 \mathrm{~K}$ collaboration. Figure taken from Ref. [5].

complicated interaction that can be accommodated in the current paradigm. The results of this section are published in Ref. [13. 


\subsubsection{Theoretical background}

Neutrino non-standard interactions with matter are generically parameterized in terms of a low-energy effective four-fermion Lagrangian 27, 225, 227]:

$$
\mathcal{L}_{\mathrm{NC}-\mathrm{NSI}}=-2 \sqrt{2} G_{F} \epsilon_{\alpha \beta}^{f X}\left(\bar{\nu}_{\alpha} \gamma^{\mu} P_{L} \nu_{\beta}\right)\left(\bar{f} \gamma_{\mu} P_{X} f\right),
$$

where $P_{X}$ denotes the left and right chirality projection operators $P_{R, L}=(1 \pm$ $\left.\gamma_{5}\right) / 2$ and $G_{F}$ is the Fermi constant. The dimensionless coefficients $\epsilon_{\alpha \beta}^{f X}$ quantify the strength of the NSI between neutrinos of flavor $\alpha$ and $\beta$ and the fundamental fermion $f \in\{e, u, d\}$.

In the presence of such new non-standard interactions of neutrinos with matter, the effective two-neutrino Hamiltonian governing neutrino propagation in the $\mu-\tau$ sector takes the form

$$
H=\frac{1}{2 E}\left[U\left(\begin{array}{cc}
0 & 0 \\
0 & \Delta m^{2}
\end{array}\right) U^{\dagger}+A\left(\begin{array}{cc}
\epsilon_{\mu \mu}^{m} & \epsilon_{\mu \tau}^{m *} \\
\epsilon_{\mu \tau}^{m} & \epsilon_{\tau \tau}^{m}
\end{array}\right)\right]
$$

where

$$
U=\left(\begin{array}{cc}
\cos \theta & \sin \theta \\
-\sin \theta & \cos \theta
\end{array}\right)
$$

represents the leptonic mixing parameterized by the mixing angle in vacuum $\theta, E$ is the neutrino energy and $A=2 \sqrt{2} G_{F} N_{e} E$ is the matter potential depending on the electron number density, $N_{e}$, along the neutrino trajectory. The parameters $\epsilon_{\mu \mu}^{m}, \epsilon_{\mu \tau}^{m}$ and $\epsilon_{\tau \tau}^{m}$ give the relative strength of the non-standard interactions compared to the Standard Model weak interactions. The superscript $m$ indicates that these parameters describe non-standard neutrino matter effects in a medium. In this case, the relevant effective operator corresponds to the vector part of the interaction described in Eq. 6.3, namely $\epsilon_{\alpha \beta}^{f V}=\epsilon_{\alpha \beta}^{f L}+\epsilon_{\alpha \beta}^{f R}$. Considering a medium formed by first generation fermions, the effective NSI coupling in matter affecting neutrino propagation is given by

$$
\epsilon_{\alpha \beta}^{m} \equiv \epsilon_{\alpha \beta}^{e V}+\frac{N_{u}}{N_{e}} \epsilon_{\alpha \beta}^{u V}+\frac{N_{d}}{N_{e}} \epsilon_{\alpha \beta}^{d V},
$$

with $N_{f}$ being the number density for the fermion $f \in\{e, u, d\}$. The flavor changing NSI parameters $\epsilon_{\alpha \beta}^{m}(\alpha \neq \beta)$ can in general be complex, while the flavor conserving coefficients $\epsilon_{\alpha \alpha}^{m}$ have to be real to preserve the hermiticity of the Hamiltonian. On the other hand, diagonal terms in the Hamiltonian proportional to the identity matrix do not affect the neutrino oscillation probability and, therefore, 
one can subtract $\epsilon_{\mu \mu}^{m}$ from the diagonal in the Hamiltonian in Eq. 6.4. In consequence, oscillation experiments are only sensitive to the combination $\epsilon_{\tau \tau}^{m}-\epsilon_{\mu \mu}^{m}$. For simplicity, and given the existence of stronger constraints on the NSI couplings for muon neutrinos compared to tau neutrinos, in the following we will set $\epsilon_{\mu \mu}^{m}=0$.

\subsubsection{Analytical results at the probability level}

In the two-neutrino approximation, the neutrino survival probability for muon neutrinos in matter of constant density is given by

$$
P_{\mu \mu}=1-\sin ^{2} 2 \theta_{m} \sin ^{2}\left(\frac{\Delta m_{m}^{2} L}{4 E}\right)
$$

where $\theta_{m}$ and $\Delta m_{m}^{2}$ are the effective mixing angle and effective mass squared difference in matter, respectively. Since there is no CP-phase in the two-neutrino picture, and, since we focus on the 2-3 sector, the oscillation probability is the same for neutrinos and antineutrinos. However, this is not true if non-standard neutrino interactions are present, since they affect differently neutrinos and antineutrinos. This difference results basically in a shift in the effective neutrino and antineutrino oscillation parameters, so for neutrinos one would have

$$
\begin{aligned}
& \Delta m_{\nu}^{2} \cos 2 \theta_{\nu}=\Delta m^{2} \cos 2 \theta+\epsilon_{\tau \tau}^{m} A, \\
& \Delta m_{\nu}^{2} \sin 2 \theta_{\nu}=\Delta m^{2} \sin 2 \theta+2 \epsilon_{\mu \tau}^{m} A .
\end{aligned}
$$

Similarly, for antineutrinos we write

$$
\begin{aligned}
& \Delta m_{\bar{\nu}}^{2} \cos 2 \theta_{\bar{\nu}}=\Delta m^{2} \cos 2 \theta-\epsilon_{\tau \tau}^{m} A, \\
& \Delta m_{\bar{\nu}}^{2} \sin 2 \theta_{\bar{\nu}}=\Delta m^{2} \sin 2 \theta-2 \epsilon_{\mu \tau}^{m} A .
\end{aligned}
$$

Here the subscript $\nu(\bar{\nu})$ indicates the effective mixing parameters in matter for neutrinos (antineutrinos). It is straightforward to see that these equations can be rearranged to express the unknown parameters $\epsilon_{\mu \tau}^{m} A, \epsilon_{\tau \tau}^{m} A, \Delta m^{2}$ and $\sin 2 \theta$, i.e. the "physical" parameters, in terms of the four observables $\Delta m_{\nu}^{2}, \Delta m_{\bar{\nu}}^{2}, \sin 2 \theta_{\nu}$ 
and $\sin 2 \theta_{\bar{\nu}}$ :

$$
\begin{aligned}
4 \Delta m^{4} & =\Delta m_{\nu}^{4}+\Delta m_{\bar{\nu}}^{4}+2 \Delta m_{\nu}^{2} \Delta m_{\bar{\nu}}^{2} \cos \left(2 \theta_{\nu}-2 \theta_{\bar{\nu}}\right) \\
\sin ^{2} 2 \theta & =\frac{\left(\Delta m_{\nu}^{2} \sin 2 \theta_{\nu}+\Delta m_{\bar{\nu}}^{2} \sin 2 \theta_{\bar{\nu}}\right)^{2}}{\Delta m_{\nu}^{4}+\Delta m_{\bar{\nu}}^{4}+2 \Delta m_{\nu}^{2} \Delta m_{\bar{\nu}}^{2} \cos \left(2 \theta_{\nu}-2 \theta_{\bar{\nu}}\right)}, \\
2 A \epsilon_{\tau \tau}^{m} & =\Delta m_{\nu}^{2} \cos 2 \theta_{\nu}-\Delta m_{\bar{\nu}}^{2} \cos 2 \theta_{\bar{\nu}}, \\
4 A \epsilon_{\mu \tau}^{m} & =\Delta m_{\nu}^{2} \sin 2 \theta_{\nu}-\Delta m_{\bar{\nu}}^{2} \sin 2 \theta_{\bar{\nu}} .
\end{aligned}
$$

From these formulas, it is obvious that a measurement of different neutrino and antineutrino mass splittings and/or mixing angles can in principle be explained by neutrino NSI with matter without resorting to CPT violation. Indeed, this idea was used to interpret different measurements in the neutrino and the antineutrino channel in the MINOS experiment [228], although this result was not confirmed by more precise data from MINOS. However, since there are already significant bounds on the values of $\epsilon_{\tau \tau}^{m}$ and $\epsilon_{\mu \tau}^{m}$, it is also clear that there will be regions in the NSI parameter space which are excluded and, then, an experimental preference for such values would rather indicate a signal of CPT violation.

Going back to Eq. (6.14), if we assume for simplicity the same mixing angles for neutrinos and antineutrinos, $\theta_{\nu}=\theta_{\bar{\nu}}$, this equation can be rewritten to

$$
\Delta\left(\Delta m^{2}\right)=\frac{2 A \epsilon_{\tau \tau}^{m}}{\cos 2 \theta}
$$

Therefore, in the case of equal mixing angles, we obtain a very simple equation for $\Delta\left(\Delta m^{2}\right)$, which is linear in $\epsilon_{\tau \tau}^{m}$. In consequence, it is straightforward to interpret a different measurement of the neutrino and antineutrino mass splittings as caused by the presence of neutrino NSI with matter. Nevertheless, as mentioned before, there are experimental bounds available on the NSI couplings that should be taken into account. In Ref. 162 one finds all the current bounds on the NSI parameters. Considering the definition of the effective NSI couplings in Eq. 6.6, the experimental bound on the diagonal NSI coupling is

$$
\left|\epsilon_{\tau \tau}^{m}\right|<0.11 \text {, at } 90 \% \text { C.L., }
$$

obtained in Ref. [229] from the analysis of atmospheric and MINOS long-baseline data. Assuming a Gaussian distribution, we can extrapolate the previous bound to other confidence levels 


$$
\left|\epsilon_{\tau \tau}^{m}\right|<n(0.067) \text {, at } n \sigma \text { C.L. }
$$

Therefore, in the presence of NSI, and according to Eq. 6.16), one can expect a difference in the effective values of the mass splitting measured in the neutrino and antineutrino channel. The size of this deviation is restricted by the existing bounds on the NSI couplings. For instance, at $n \sigma$ C.L., the maximum deviation will be given by

$$
\Delta\left(\Delta m^{2}\right) \leq \frac{2 A}{\cos 2 \theta} \times n(0.067),
$$

where we have taken

$$
A=2.27 \times 10^{-4} \mathrm{eV}^{2}\left(\frac{E}{\mathrm{GeV}}\right) .
$$

That corresponds to the density of the Earth crust, $\rho=3 \mathrm{~g} / \mathrm{cm}^{3}$. This result also applies to the flavor violating NSI coupling $\epsilon_{\mu \tau}^{m}$. In this case, the current bound is given by

$$
\left|\epsilon_{\mu \tau}^{m}\right|<0.018 \text { at } 90 \% \text { C.L., }
$$

originally given in Refs. 230,231. Therefore, assuming equal mixing angles in both sectors, the deviation NSI can induce on the neutrino mass splitting over the antineutrino one can be expressed as

$$
\Delta\left(\Delta m^{2}\right)=\frac{4 A \epsilon_{\mu \tau}^{m}}{\sin 2 \theta} \leq \frac{4 A}{\sin 2 \theta} \times n(0.011) .
$$

The allowed deviations between the neutrino and antineutrino mass splitting as a function of the NSI coupling $\epsilon_{\tau \tau}^{m}\left(\epsilon_{\mu \tau}^{m}\right)$ are shown in the left (right) panel of Fig. 6.9. There, we have chosen $\theta=41^{\circ}$ and the energies $E=1.0,2.5,10 \mathrm{GeV}$, being $2.5 \mathrm{GeV}$ the peak energy for $\nu_{e}$ appearance at DUNE. From this figure, one can read to which amount a measurement of $\Delta\left(\Delta m^{2}\right)$ different from zero could be induced by NSI instead of being a signal of intrinsic CPT violation. The vertical dashed black lines indicate the current (1-4) $\sigma$ bounds on $\epsilon_{\tau \tau}^{m}$ and $\epsilon_{\mu \tau}^{m}$. The difference in the slope of the two graphs can be understood from two facts: first, the bound on $\epsilon_{\mu \tau}^{m}$ is stronger than the one for $\epsilon_{\tau \tau}^{m}$ and, second, for the mixing angle of choice, we have $\cos 2 \theta \approx 0.14$ and $\sin 2 \theta \approx 0.99$, so the deviation potentially induced by $\epsilon_{\tau \tau}^{m}$ can be much larger. Note that, in this section, we have assumed equal mixing angles, so that the situation could be even more confusing if one allows for different mixing angles in the neutrino and antineutrino sector. Notice 

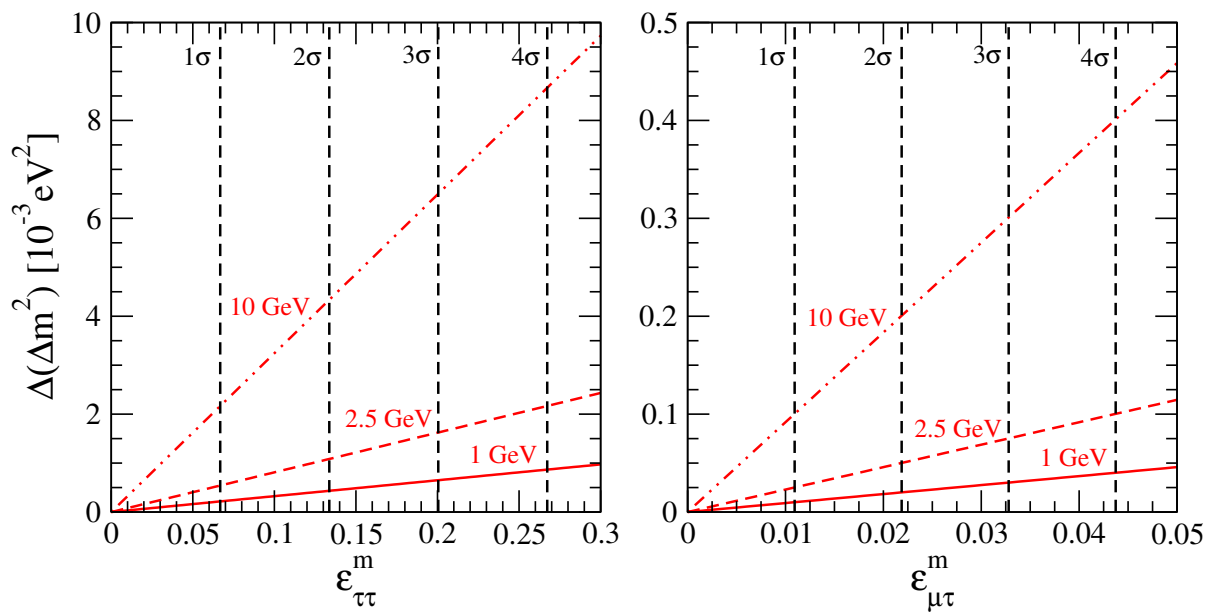

Figure 6.9: Left: $\Delta\left(\Delta m^{2}\right)$ as a function of $\epsilon_{\tau \tau}^{m}$, showing the allowed deviations of $\Delta\left(\Delta m^{2}\right)$ that could be explained with NSI for $\theta=41^{\circ}$ and $E=1.0 \mathrm{GeV}$ (solid), $E=2.5 \mathrm{GeV}$ (dashed) and $E=10 \mathrm{GeV}$ (dot-dashed line). Right: The same for $\Delta\left(\Delta m^{2}\right)$ as a function of $\epsilon_{\mu \tau}^{m}$. Figure taken from Ref. 13 .

also that these results have been derived at the probability level and, in principle, one can expect the picture to be somehow blurred when the complete analysis of a neutrino experiment is carried out taking into account the full simulated neutrino spectrum and the associated statistical and systematical errors.

\subsubsection{Results from the simulation of DUNE}

We have shown that, if the results from the separate neutrino and antineutrino analysis of the T2K collaboration 103 turn out to be true, DUNE could measure CPT violation at more than $3 \sigma$. In this section, we will analyze if indeed these results could be confused with NSI performing the full DUNE simulation.

The simulation of the experiment is performed as in Sec.6.1.1. However, since here we focus on the parameters relevant for $P_{\mu \mu}$, we consider only the disappearance channel in our simulation this time. When considering non-standard interactions the DUNE signal is simulated using the GLoBES extension snu.c [58,232].

We again perform two simulations of DUNE running 3.5 years only in neutrino mode and 3.5 years only in antineutrino mode. To generate the future data, we assume the parameters presented in Tab. 6.2, assuming different atmospheric mixing angle and mass splitting for neutrinos and antineutrinos, but no NSI. Then, in the statistical analysis, we scan over the relevant oscillation 
parameters $\delta, \theta_{13}, \theta_{23}, \Delta m_{31}^{2}$ and their antineutrino counterparts. We again put a prior on $\bar{\theta}_{13}$, due to the precise measurements by the short baseline reactor experiments [86, 91, 137]. Additionally, we scan over the two NSI parameters in Eq. (6.4), $\epsilon_{\tau \tau}^{m}$ and $\epsilon_{\mu \tau}^{m}$, setting the rest of the parameters including all of the new phases to zero. In this way, we obtain two $\chi^{2}$ grids, one for neutrinos and another one for antineutrinos. Then, we try to reconstruct the oscillation parameters under the hypothesis of CPT invariance. To do so we calculate the total $\chi^{2}$ distribution from the sum of the neutrino and antineutrino disappearance contributions

$$
\chi_{\text {total }}^{2}=\chi^{2}(\nu)+\chi^{2}(\bar{\nu})
$$

and then marginalize over all the parameters except the one of interest. First of all, we focus our attention to the NSI parameter $\epsilon_{\mu \tau}^{m}$. The $\Delta \chi^{2}$ profile obtained for this coupling is the orange line in the left panel of Fig. 6.10. Here we see that data are consistent with this NSI coupling equal to zero. However, the result is different for the flavor diagonal NSI coupling $\epsilon_{\tau \tau}^{m}$. The $\Delta \chi^{2}$ profile for this parameter is plotted in the right panel of Fig. 6.10 (orange line). From the figure, we see our analysis excludes the value $\epsilon_{\tau \tau}^{m}=0$ at approximately $4 \sigma$, while the best fit is obtained for $\epsilon_{\tau \tau}^{m}=-0.33$. Besides the absolute minimum, three other local minima are obtained with $\Delta \chi^{2}<0.4$. This multiplicity is due to the octant degeneracy present in the analysis of the disappearance channel alone. As it is well known, disappearance data are mostly sensitive to $\sin ^{2} 2 \theta_{23}$ and, therefore, can not distinguish the correct octant of $\theta_{23}$. In this case, the degeneracy in the determination of the atmospheric mixing angle appears twice, for the neutrino and the antineutrino mixing angle, and two different values of $\cos 2 \theta_{\nu}$ and $\cos 2 \theta_{\bar{\nu}}$ are preferred at the same confidence level. As a result, and as it can be easily understood from Eq. (6.14), four different values of $\epsilon_{\tau \tau}^{m}$ are allowed at roughly the same confidence level. This degeneracy could be partially broken by including the appearance channel in our analysis. However, doing this, we would also have to include more NSI parameters in the analysis, since other NSI parameters as $\epsilon_{e \mu}^{m}$ and $\epsilon_{e \tau}^{m}$ will be very relevant for the appearance channel. Our results can be explained from the naive formulae in Eqs. ( (6.14) $)$ and $(6.15)$. Indeed, if we substitute the T2K best fit values for the oscillation parameters given in Table 6.2, used to simulate future DUNE data, into these formulae, for the peak energy of DUNE, $E=2.5 \mathrm{GeV}$, we obtain $\epsilon_{\mu \tau}^{m}=5.8 \times 10^{-3}$ and $\epsilon_{\tau \tau}^{m}=-0.42$. Therefore, one can conclude that what seems to be CPT violation could be also explained with neutrino NSI with matter. This can also be illustrated through the energy distribution of the number of expected events in DUNE for both scenarios, as shown in Fig. 6.11. The left panel corresponds 



Figure 6.10: $\Delta \chi^{2}$ profile for the NSI couplings $\epsilon_{\mu \tau}^{m}$ (left) and $\epsilon_{\tau \tau}^{m}$ (right panel) obtained in our analysis (orange lines). For comparison we show the current experimental bounds (see Eqs. (6.17) and (6.21) and the corresponding discussions) on both parameters assuming Gaussian errors (black lines). Figure taken from Ref. [13].

to the event distribution for neutrinos (blue) and antineutrinos (red), assuming CPT violation, with the oscillation parameters for neutrinos and antineutrinos given in Tab. 6.2. The right panel, on the contrary, shows the spectrum of events expected in the CPT-conserving case with NSI. It should be noted that, for this last scenario, we perform a combined analysis of the neutrino and antineutrino channel assuming equal values for the oscillation parameters. As a result, we not only obtain a non-zero coefficient $\epsilon_{\tau \tau}^{m}$, but also different standard oscillation parameters from the ones used to simulate the neutrino signal in DUNE ${ }^{4}$ Indeed, a new best fit point is found, located at $\epsilon_{\tau \tau}^{m}=-0.33, \sin ^{2} \theta_{23}^{\text {comb }}=0.56$, $\sin ^{2} \theta_{13}^{\mathrm{comb}}=0.0216, \Delta m_{31}^{2 \mathrm{comb}}=2.62 \times 10^{-3} \mathrm{eV}^{2}$ and $\delta^{\mathrm{comb}}=1.4 \pi$. As one can see, both panels are almost identical, showing the equivalence between the two scenarios under analysis. As a final remark, we would like to add that the two analyzed possibilities can not be distinguished by means of $\chi^{2}$ either. Since we create fake data under the assumption of $\mathrm{CPT}$ violation, the best fit point for the independent analysis of neutrino and antineutrino data automatically gets the value $\chi^{2}=0$. However, when we perform a CPT-conserving analysis with NSI,

\footnotetext{
${ }^{4}$ We had already discussed this type of imposter solutions in Sec. 6.1.3
} 

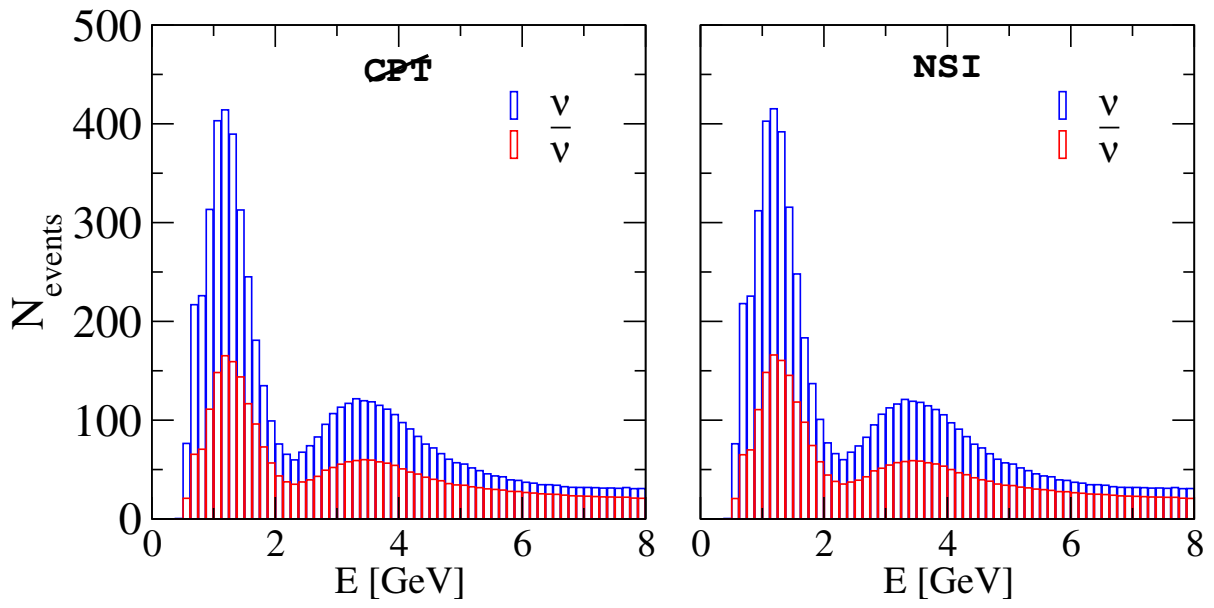

Figure 6.11: Number of muon neutrino (blue) and muon antineutrino (red) events for CPT violation (left) and for the CPT-conserving scenario with NSI (right). The parameters used to create the plot in the CPT-violating case are the ones of Tab. 6.2. For the NSI case, we used the combined new best fit, namely $\epsilon_{\tau \tau}^{m}=$ $-0.33, \sin ^{2} \theta_{23}^{\text {comb }}=0.56, \sin ^{2} \theta_{13}^{\text {comb }}=0.0216, \Delta m_{31}^{2}{ }^{\text {comb }}=2.62 \times 10^{-3} \mathrm{eV}^{2}$ and $\delta^{\mathrm{comb}}=1.4 \pi$. Figure taken from Ref. $[13$.

the new best fit point has a value of only $\chi^{2}=0.55$ and, therefore, one can not claim the fit to data is significantly worse in that case.

Nevertheless, it should be noted that the best fit value we have obtained in this NSI case for the flavor diagonal coupling, $\epsilon_{\tau \tau}^{m}=-0.33$, is highly excluded from current experimental data. To visualize this, together with the results obtained in our analysis, we have plotted in Fig. 6.10 the profile for both NSI parameters from current data assuming Gaussian errors (see black lines in both panels). In the right graph, one can see that our best fit value for $\epsilon_{\tau \tau}^{m}$ is actually excluded at close to $5 \sigma$. Nevertheless, some of the values remain allowed at $2 \sigma$ level, as can be seen in the figure. Note, however, that we plot the current bounds on $\epsilon_{\tau \tau}^{m}$ and $\epsilon_{\mu \tau}^{m}$, which will also further improve in the future. Therefore, if the results from T2K (that we take as input parameters for the simulation of the CPT-violating data sample in DUNE) turn out to be true, they could hint towards CPT violation rather than to NSI. 



\section{Chapter 7}

\section{Quasi-Dirac neutrino oscillations at DUNE and JUNO}

This chapter is dedicated to a sensitivity study of the DUNE and JUNO experiments to quasi-Dirac neutrino oscillations. The presence of Majorana and Dirac mass terms in the Lagrangian density produces new mixing angles and mass splittings which can alter the standard oscillations. The results of this chapter are published in Ref. [15.

\section{$7.1 \quad$ Introduction}

Since neutrino oscillations are blind to the Dirac or Majorana nature of neutrinos, one needs other types of experiments, for example, those searching for neutrinoless double beta decay to determine it 233, 234. In general, one can say that the Dirac case, consisting of $n$ neutrinos, is a limiting case of the more general Majorana scenario, with $2 n$ neutrinos. This limit is performed by putting the Majorana mass terms in the Lagrangian to zero. Quasi-Dirac neutrinos arise from the presence of both Majorana and Dirac mass terms in the Lagrangian simultaneously, where the Majorana terms are small, but not exactly zero. As we will show, the departure from Diracness - i.e. non-zero Majorana mass terms leads to the presence of new mixing angles and new mass splittings, which will affect neutrino oscillation probabilities.

Let us clarify that, we will use "quasi-Dirac neutrinos" to refer to active-sterile neutrino pairs 235 . In order to distinguish this scenario from the one with active- 
active pairs, we denote the latter ones as pseudo-Dirac neutrinos 236. Many aspects of pseudo-Dirac neutrinos have been studied in the literature, see for example Refs. 237 244]. Note, however, that models with pseudo-Dirac neutrinos do not fit oscillation data anymore 245 247]. In the context of quasi-Dirac neutrinos, many papers appeared in the literature proposing explanations for the solar and atmospheric neutrino problems [248 250], as well as consistent descriptions of standard and short baseline neutrino oscillations 251,252 . Several papers derived limits on quasi-Dirac neutrino properties from different data sets [253.254], while others discussed them in the context of neutrino telescopes 255 258.

From a theoretical point of view, there are several options on how quasi-Dirac neutrinos can be created. They can be produced, for instance, in models with a singular seesaw 259 260], double seesaw [261] or Dirac-seesaw 262] mechanisms. Another possibility is to obtain them from extended gauge groups 263, 264 or even in super-gravity theories [265].

Because of the presence of new spinors in Dirac neutrino models, there is some overlap between the study of quasi-Dirac neutrinos and the scenario with sterile neutrinos. Several experimental hints point towards the existence of sterile neutrinos, which have been extensively investigated in many experiments. The possible observation of short baseline oscillations in some of these experiments 266 273] \begin{tabular}{ll|l|l|l|l|l|l|l|l|}
\hline together with the non-observation of neutrino oscillations in others & 23 & 274 & 283 \\
\hline
\end{tabular} lead to large tensions in the global $3+1$ picture 284288 . Even though there is some theoretical overlap, these results would point towards new mass splittings at the $\sim 1 \mathrm{eV}^{2}$ scale. Therefore, this type of oscillations cannot be explained with quasi-Dirac neutrinos, whose additional mass splittings are constrained to be much below the eV scale.

In this chapter, we study the sensitivity of DUNE and JUNO to quasi-Dirac neutrino oscillations.

\subsection{Quasi-Dirac neutrino oscillations}

A pair of quasi-Dirac neutrinos is a pair of Majorana neutrinos with a small mass splitting and a relative CP-sign between the two states. For the sake of illustration, let us start considering only one neutrino generation. In this case, in the basis $\left(\nu, N^{c}\right)$, where $\nu$ and $N^{c}$ are the active and the sterile neutrinos, respectively, the most general neutrino mass matrix is

$$
m_{\nu}=\left(\begin{array}{cc}
m_{L} & m_{D} \\
m_{D} & m_{R}
\end{array}\right)
$$


Here, $m_{L}$ and $m_{R}$ are the terms that violate lepton number, while $m_{D}$ is the standard Dirac neutrino mass term. In the limit in which $m_{L}$ and $m_{R}$ are equal to zero, lepton number is conserved and neutrinos are Dirac particles. This limiting case is characterized by two degenerate mass eigenstates

$$
\begin{aligned}
& \nu_{1}=\frac{1}{\sqrt{2}}\left(\nu+N^{c}\right), \\
& \nu_{2}=\frac{i}{\sqrt{2}}\left(-\nu+N^{c}\right),
\end{aligned}
$$

where the factor $i$ is introduced such that both mass eigenvalues are positive. Note that, in this mass eigenstate basis, both $\nu_{1}$ and $\nu_{2}$ are equal mixtures of active and sterile neutrinos. Small deviations from the limit $m_{L}=m_{R}=0$ then lead to quasi-Dirac neutrinos. If we define the new variables $\varepsilon=\left(m_{L}+m_{R}\right) /\left(2 m_{D}\right)$ and $\theta=\left(m_{L}-m_{R}\right) /\left(4 m_{D}\right)$, in the limit $\varepsilon, \theta \ll 1$, one can rewrite Eq. 7.2$)$ as

$$
\begin{aligned}
& \nu_{1} \simeq \frac{1}{\sqrt{2}}\left[(1+\theta) \nu+(1-\theta) N^{c}\right], \\
& \nu_{2} \simeq \frac{i}{\sqrt{2}}\left[(-1+\theta) \nu+(1+\theta) N^{c}\right],
\end{aligned}
$$

where the quasi-degenerate pairs are nearly maximally mixed and $\theta$ is a small angle describing the departure from maximality. The masses are given by

$$
m_{1,2} \simeq m_{D}(1 \pm \varepsilon) .
$$

Quasi-Dirac neutrinos are therefore characterized by new mass splittings and new mixing angles.

Let us now consider the extension of the standard model with three sterile neutrinos $N^{c}$. In the physical mass eigenstate basis, the charged current SM Lagrangian is modified to

$$
\mathcal{L}_{C C}=-\frac{g}{\sqrt{2}} W_{\mu}^{-} \sum_{l=1}^{3} \sum_{j=1}^{6} \mathbf{V}_{l j} \bar{\ell}_{l} \gamma^{\mu} P_{L} \nu_{j}+\text { h.c. },
$$

where $P_{L, R}=\left(1 \mp \gamma_{5}\right) / 2$ are the chirality projectors, $l=1,2,3$ denote the flavor of the charged leptons, and $j=1, \ldots, 6$ the physical neutrino states. The mixing is parameterized by a rectangular $3 \times 6$ mixing matrix, $\mathbf{V}_{l j} 289$. Moreover, the 
addition of the three sterile neutrinos allows for the mass term

$$
\mathcal{L}_{\text {mass }}=\frac{1}{2} \bar{\nu}_{\alpha} M_{\alpha \beta} \nu_{\beta}+\text { h.c. }
$$

Here, indices $\alpha, \beta=1,2,3(4,5,6)$ are for active (sterile) neutrinos and $M_{\alpha \beta}$ is the generalization of Eq. $(7.1)$ for three generations. The full neutrino mass matrix is now diagonalized by a $6 \times 6$ unitary matrix, $\tilde{\mathbf{U}}$. We parameterize the neutrino mixing matrix as

$$
\tilde{\mathbf{U}}\left(\theta_{i j}, \delta_{i j}\right)=\widehat{R}_{56} \widehat{R}_{46} \widehat{R}_{36} \widehat{R}_{26} \widehat{R}_{16} \widehat{R}_{45} \widehat{R}_{35} \widehat{R}_{25} \widehat{R}_{15} \widehat{R}_{34} \widehat{R}_{24} \widehat{R}_{14} \widehat{R}_{23} \widehat{R}_{13} \widehat{R}_{12},
$$

where $\widehat{R}_{i j}$ are complex rotation matrices which depend on the mixing angles $\theta_{i j}$ and CP-violating phases $\delta_{i j}$. The rotation matrices $\widehat{R}_{i j}$ are parameterized in the usual way. For example, for $\widehat{R}_{14}$ we have

$$
\widehat{R}_{14}=\left(\begin{array}{cccccc}
\cos \theta_{14} & 0 & 0 & e^{-i \delta_{14}} \sin \theta_{14} & 0 & 0 \\
0 & 1 & 0 & 0 & 0 & 0 \\
0 & 0 & 1 & 0 & 0 & 0 \\
-e^{i \delta_{14} \sin \theta_{14}} & 0 & 0 & \cos \theta_{14} & 0 & 0 \\
0 & 0 & 0 & 0 & 1 & 0 \\
0 & 0 & 0 & 0 & 0 & 1
\end{array}\right) .
$$

Note that the matrix $\tilde{\mathbf{U}}$ in Eq. 7.6 contains the mixing among sterile neutrinos, not observable in neutrino oscillation experiments. Thus, we will neglect these rotations in the following. In the remaining rotations, we have in general 12 angles and 12 phases. However, in our numerical studies we will limit ourselves to two phases only, namely $\delta_{13}$ and $\delta_{16}$. This means that the mixing matrix above can be reduced to

$$
\tilde{\mathbf{U}}\left(\theta_{i j}, \delta_{i j}\right)=R_{36} R_{26} \widehat{R}_{16} R_{35} R_{25} R_{15} R_{34} R_{24} R_{14} R_{23} \widehat{R}_{13} R_{12},
$$

where $R_{i j}$ denote real rotations. It proves convenient to multiply $\tilde{\mathbf{U}}$ by the following $6 \times 6$ rotation matrix (as in Eq. (16) of Ref. 290):

$$
\mathbf{U}\left(\theta_{i j}, \delta_{i j}\right) \equiv \tilde{\mathbf{U}}\left(\theta_{i j}, \delta_{i j}\right) W, \quad \text { with } W=\frac{1}{\sqrt{2}}\left(\begin{array}{cc}
I_{3} & i I_{3} \\
I_{3} & -i I_{3}
\end{array}\right),
$$

with $I_{3}$ being the $3 \times 3$ identity matrix. This redefinition allows to recover trivially the Dirac limit for the mixing matrix, by putting to zero all non-standard angles. The probability of a neutrino oscillating from a flavor $\alpha$ to a flavor $\beta$ can then 
be written as

$$
P\left(\nu_{\alpha} \rightarrow \nu_{\beta}\right)=\left|\sum_{j=1}^{6} \mathbf{U}_{\beta j} \mathbf{U}_{\alpha j}^{*} \exp \left(-\frac{i m_{j}^{2} L}{2 E}\right)\right|^{2},
$$

where $L$ is the length traveled by the neutrino and $E$ its energy. Therefore, neutrino oscillations are described by the Hamiltonian

$$
\mathcal{H}_{0}=\frac{1}{2 E} \mathbf{U M}^{2} \mathbf{U}^{\dagger}
$$

where $\mathbb{M}^{2}=\operatorname{diag}\left(0, \Delta m_{21}^{2}, \Delta m_{31}^{2}, \epsilon_{1}^{2}, \Delta m_{21}^{2}+\epsilon_{2}^{2}, \Delta m_{31}^{2}+\epsilon_{3}^{2}\right)^{1}$ and the square of the lightest neutrino mass, $m_{1}^{2}$, has been subtracted from the diagonal elements in the $\mathbb{M}^{2}$ matrix, as usual. To include matter effects on the neutrino propagation, one should add the effective matter potential to the neutrino Hamiltonian above. Quasi-Dirac neutrinos feel the same potential in the 4-5-6 sector as in the 1-2-3 sector. Thus,

$$
\mathcal{H}=\frac{1}{2 E}\left(\mathbf{U M}^{2} \mathbf{U}^{\dagger}+\mathbb{A}\right),
$$

where the potential is now given by $\mathbb{A}=\operatorname{diag}\left(V_{\mathrm{CC}}+V_{\mathrm{NC}}, V_{\mathrm{NC}}, V_{\mathrm{NC}}, V_{\mathrm{CC}}+\right.$ $\left.V_{\mathrm{NC}}, V_{\mathrm{NC}}, V_{\mathrm{NC}}\right)$. The charged current potential is given by $V_{\mathrm{CC}}=2 E \sqrt{2} G_{F} n_{e}$, where $G_{F}$ is the Fermi constant and $n_{e}$ is the electron number density. The neutral current potential, $V_{\mathrm{NC}}$, is a common term to all the diagonal entries and, therefore, it can be removed from the effective Hamiltonian, that will read as follows

$$
\mathbb{A}=\operatorname{diag}\left(V_{\mathrm{CC}}, 0,0, V_{\mathrm{CC}}, 0,0\right) .
$$

This Hamiltonian will lead to a different oscillation behavior compared to the standard case, as soon as any $\epsilon_{i}$ or any non-standard mixing angle is different from zero. As an example, we show in Fig. 7.1 (top panels) the oscillation probabilities for the two channels relevant for DUNE, $\nu_{\mu} \rightarrow \nu_{e}$ and $\nu_{\mu} \rightarrow \nu_{\mu}$. The standard oscillation parameters in these plots are fixed to the ones in Tab. 7.1. In the left panel we show the disappearance probability $P_{\mu \mu}$ as a function of the neutrino energy, turning on one new mixing angle at a time - which is always set to $\sin ^{2} \theta_{\text {new }}=0.2$. The new angle $\theta_{16}$ has no visible effect on the disappearance probability, while $\theta_{26}$ has a visible effect close to the oscillation minima. In the right panel of Fig. 7.1 we show the appearance probability $P_{\mu e}$. Here both angles

\footnotetext{
${ }^{1}$ From this expression it is clear why the convention chosen in Eq. $\sqrt{7.9}$ is useful. Setting $\epsilon_{i}^{2}$ and the non-standard angles to zero, $P\left(\nu_{\alpha} \rightarrow \nu_{\beta}\right)$ reduces to the standard expression for three generations, despite the fact that we sum over six states.
} 

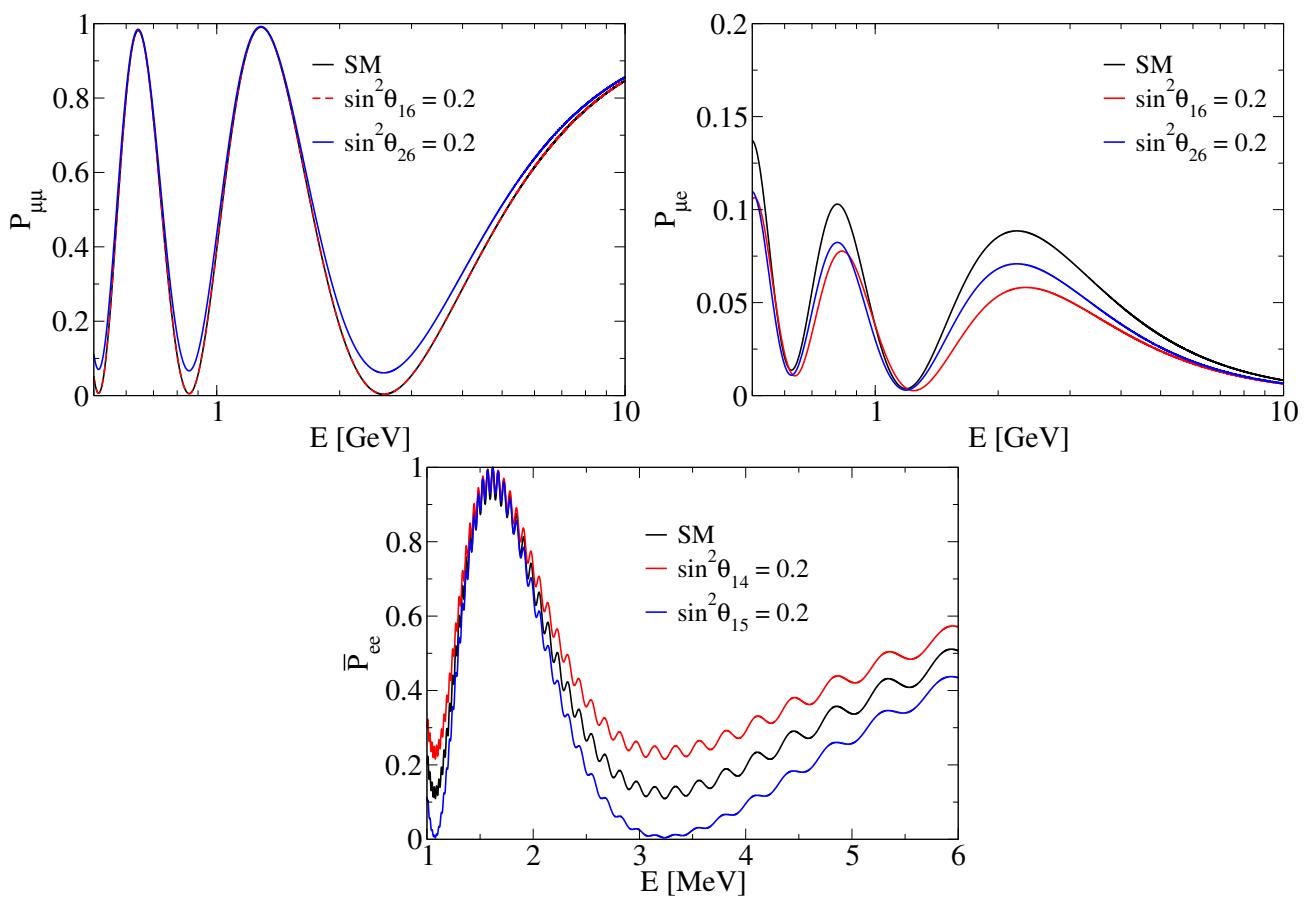

Figure 7.1: Disappearance (upper left) and appearance (upper right) probability, $P_{\mu \mu}$ and $P_{\mu e}$, at DUNE as a function of the neutrino energy. Lower panel: antineutrino survival probability, $\bar{P}_{e e}$, in JUNO. In all cases, the black line corresponds to the expected probability in the SM with only three active neutrinos, while the red and blue curves are obtained setting a new mixing angle in the quasi-Dirac scenario to the value indicated in the legend. All the other new mixing angles are set to zero. Figure taken from Ref. 15 .

have a visible impact in the oscillation probability. This is expected from the fact that the new angles $\theta_{16}$ and $\theta_{26}$ take the role of the standard angles $\theta_{13}$ and $\theta_{23}$, respectively. On the other hand, the lower panel of Fig. 7.1 shows the effect of the new mixing angles $\theta_{14}$ and $\theta_{15}$ on the survival probability of electron antineutrinos at JUNO. In this case, the two mixing angles have opposite effects. Note that the survival probability shown here does not include the experimental energy resolution, which is included in our simulation of JUNO in Sec. 7.3.

Fig.7.1 is meant for illustration purposes only: as we shall see later in Sec. 7.4. standard and non-standard angles are highly correlated in the quasi-Dirac neutrino scenario and one can obtain perfect degeneracies among certain parameters. 


\begin{tabular}{|c|c|}
\hline Parameter & Value \\
\hline$\Delta m_{21}^{2}$ & $7.55 \times 10^{-5} \mathrm{eV}^{2}$ \\
$\Delta m_{31}^{2}$ & $2.50 \times 10^{-3} \mathrm{eV}^{2}$ \\
$\sin ^{2} \theta_{12}$ & 0.32 \\
$\sin ^{2} \theta_{23}$ & 0.547 \\
$\sin ^{2} \theta_{13}$ & 0.0216 \\
$\delta$ & $1.5 \pi$ \\
\hline
\end{tabular}

Table 7.1: Standard neutrino oscillation parameters used in the analysis.

That is, even very different combinations of angles can lead to similar oscillation probabilities, which makes the establishment of limits on quasi-Dirac angles particularly difficult experimentally. For this reason, Ref. [290] introduced a particular set of variables, $X_{i}$, which are parametrization independent combinations of entries in the neutrino mixing matrix $\mathbf{U}$. Not considering transitions to $\nu_{\tau}$, due to the scarcity of $\nu_{\tau}$ appearance data, one can show that only seven independent combinations of neutrino mixing angles enter the oscillation probabilities. The corresponding $X_{i}$ are defined as

$$
\begin{aligned}
& X_{1}=\left|\mathbf{U}_{e 3}\right|^{2}+\left|\mathbf{U}_{e 6}\right|^{2}, X_{2}=\left|\mathbf{U}_{e 2}\right|^{2}+\left|\mathbf{U}_{e 5}\right|^{2}, \\
& X_{3}=\left|\mathbf{U}_{\mu 3}\right|^{2}+\left|\mathbf{U}_{\mu 6}\right|^{2}, X_{4}=\left|\mathbf{U}_{\mu 2}\right|^{2}+\left|\mathbf{U}_{\mu 5}\right|^{2}, \\
& X_{5}=\left|\mathbf{U}_{e 3} \mathbf{U}_{\mu 3}^{*}+\mathbf{U}_{e 6} \mathbf{U}_{\mu 6}^{*}\right|^{2}, X_{6}=\left|\mathbf{U}_{e 2} \mathbf{U}_{\mu 2}^{*}+\mathbf{U}_{e 5} \mathbf{U}_{\mu 5}^{*}\right|^{2}, \\
& X_{7}=\left(\mathbf{U}_{e 3} \mathbf{U}_{\mu 3}^{*}+\mathbf{U}_{e 6} \mathbf{U}_{\mu 6}^{*}\right)\left(\mathbf{U}_{e 2} \mathbf{U}_{\mu 2}^{*}+\mathbf{U}_{e 5} \mathbf{U}_{\mu 5}^{*}\right),
\end{aligned}
$$

where $\mathbf{U}$ is the full mixing matrix defined in Eq. (7.9). Note that $\left|X_{7}\right|^{2}=X_{5} X_{6}$, i.e. only the phase in $X_{7}$ is a free parameter. The oscillation probabilities in vacuum can be written in terms of the $X_{i}$ as 290

$$
\begin{aligned}
P\left(\nu_{e} \rightarrow \nu_{e}\right) & =1+\left(1-X_{1}-X_{2}\right) X_{2} \mathcal{A}_{21} \\
& +\left(1-X_{1}-X_{2}\right) X_{1} \mathcal{A}_{31}+X_{1} X_{2} \mathcal{A}_{32} \\
P\left(\nu_{\mu} \rightarrow \nu_{\mu}\right) & =1+\left(1-X_{3}-X_{4}\right) X_{4} \mathcal{A}_{21} \\
& +\left(1-X_{3}-X_{4}\right) X_{3} \mathcal{A}_{31}+X_{3} X_{4} \mathcal{A}_{32} \\
P\left(\nu_{e} \rightarrow \nu_{\mu}\right) & =-\left(X_{6}+\operatorname{Re} X_{7}\right) \mathcal{A}_{21}-\left(X_{5}+\operatorname{Re} X_{7}\right) \mathcal{A}_{31} \\
& +\operatorname{Re} X_{7} \mathcal{A}_{32}+\operatorname{Im} X_{7}\left(\mathcal{B}_{21}-\mathcal{B}_{31}+\mathcal{B}_{32}\right)
\end{aligned}
$$

where $\mathcal{A}_{i j} \equiv-4 \sin ^{2}\left[\left(m_{i}^{2}-m_{j}^{2}\right) L /(4 E)\right]$ and $\mathcal{B}_{i j} \equiv 2 \sin \left[\left(m_{i}^{2}-m_{j}^{2}\right) L /(2 E)\right]$. 
The usefulness of defining these $X_{i}$ lies in the fact that, for a three-generation Dirac scenario, there are only four independent parameters entering these seven quantities: the three standard mixing angles and the phase $\delta_{13}$. Thus, in the Dirac limit, one can find three relations among the seven $X_{i}$ :

$$
\begin{gathered}
X_{5}=X_{1} X_{3}, \quad X_{6}=X_{2} X_{4}, \\
\operatorname{Re}\left(X_{7}\right)=\frac{1}{2}\left(1-X_{1}-X_{2}-X_{3}-X_{4}+X_{1} X_{4}+X_{2} X_{3}\right) .
\end{gathered}
$$

Eq. (7.18) allows to formulate quantitative tests of "quasi-Diracness". We will come back to this in Sec. 7.4. Note that, although there are seven $X_{i}$, DUNE will not be sensitive to $X_{1}$ and $X_{2}$, since they depend on the solar parameters. However, JUNO (and Daya Bay, whose measurement of $X_{1}$ we will include as a prior in our analysis) will provide stringent constraints on $X_{1}$ and $X_{2}$, see section 7.4. On the contrary, DUNE will be able to put severe restrictions on $X_{3}$ and $X_{5}$ and some improvements on the remaining parameters $X_{4}, X_{6}$ and $X_{7}$, as we will show below.

Beyond the new mixing angles, we show DUNE's sensitivity to the new mass splittings $\epsilon_{i}$ in Fig. 7.2. These results have been obtained by varying only one of the new mass splittings at a time and fixing the new angles to zero. In comparison with previous results derived in Ref. [290, one can see that DUNE will not be competitive with other current experiments, which give bounds on $\epsilon_{1}$ and $\epsilon_{2}$ several orders of magnitude stronger than the ones shown in Fig. 7.2, The only comparable bound is the one for $\epsilon_{3}$. Note, however, that in Ref. 290 the authors marginalized over some of the oscillation parameters, while we kept all of them fixed. Marginalizing over additional parameters would result in weaker bounds, also for $\epsilon_{3}$. Therefore, given the poor sensitivity of DUNE to the new splittings $\epsilon_{i}$, we will set them to very small values in our analysis. The sensitivity of JUNO to the mass splittings $\epsilon_{i}$ has been discussed in Ref. [290. We can infer from Tab. 1 in 290 that JUNO will neither be able to improve the current bounds on any of the new mass splittings.

\subsection{Simulation of experiments}

Our simulation of DUNE follows the description given in chapter 5 . To include quasi-Dirac neutrino oscillations in our simulation of DUNE, we have modified the GLoBES extension snu.c 58,232. Specifically, we have modified the definition of the neutrino oscillation probability function inside snu.c by adding the additional rotation matrix of Eq. (7.9) and the matter potential of Eq. (7.13). 


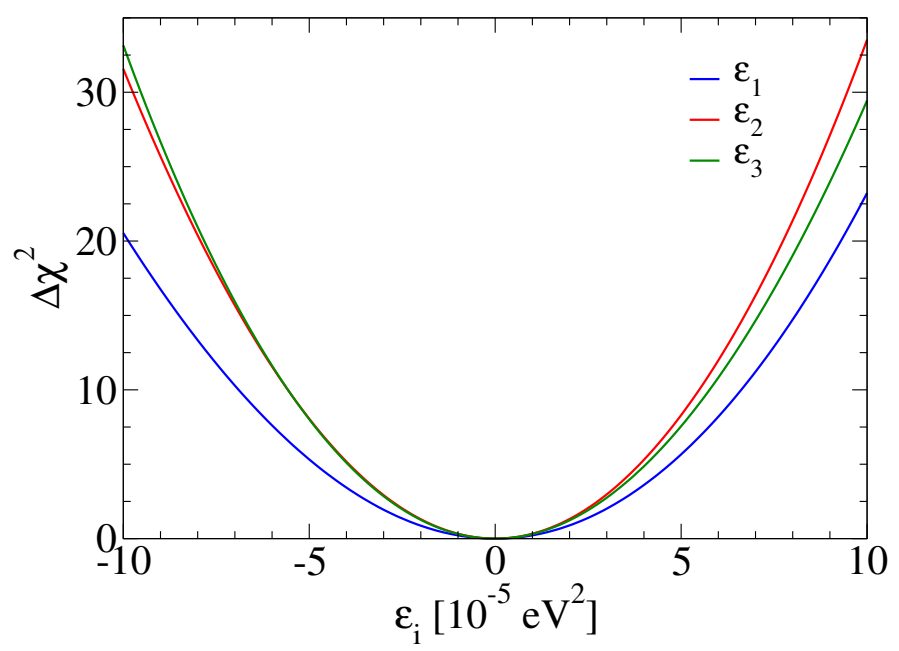

Figure 7.2: $\chi^{2}$ profiles for the new mass splittings $\epsilon_{i}$. New angles are fixed to zero. Figure taken from Ref. [15.

For the statistical analysis, we create a fake DUNE data sample using the standard oscillation parameters from Tab. 7.1. Next, we try to reconstruct the simulated data varying the mixing angles $\theta_{13}, \theta_{23}, \theta_{16}$ and $\theta_{26}$ (most relevant for DUNE) and the two CP-violating phases $\delta_{13}$ and $\delta_{16}$. The remaining new mixing angles are fixed to zero and the new mass splittings $\epsilon_{i}^{2}$ are fixed to very small values. As discussed in former chapters, DUNE has no sensitivity to the solar parameters. Therefore, they are fixed at their best fit values, in Tab. 7.1. On the other hand, given that we are mostly interested in correlations between the standard and new mixing angles, we have also kept $\Delta m_{31}^{2}$ fixed to its best fit value. Currently, there is a preference for normal mass ordering slightly above $3 \sigma\left[2 \sqrt[4]{4}\right.$, so we will not consider negative values of $\Delta m_{31}^{2}$ here. We use GLoBES to calculate the event numbers for a given set of oscillation parameters $p$ and then we calculate the $\chi^{2}$ for DUNE using Eq. (5.1).

In order to integrate JUNO in the analysis, one can realize that the factors in front of the $\mathcal{A}_{i j}$ in Eq. (7.15) are functions of the standard and new oscillation parameters. Knowing the standard sensitivity of the experiment, it is possible to parameterize the corresponding $\chi^{2}$ function, $\chi_{\mathrm{JUNO}}^{2}$. It is obtained by allowing the variation of the five parameters which are relevant for JUNO, namely $\theta_{12}, \theta_{13}, \theta_{14}, \theta_{15}$ and $\theta_{16}$. The solar mass splitting $\Delta m_{21}^{2}$ is fixed to its best fit value. 
To get the global future sensitivity to the quasi-Dirac scenario, in our analysis we combine the individual sensitivities obtained for DUNE and JUNO. Besides the two $\chi^{2}$ functions discussed above, $\chi_{\text {DUNE }}^{2}$ and $\chi_{\text {JUNO }}^{2}$, we introduce a penalty function associated to some of the mixing angles under study. As it was shown in [290], the current reactor experiments cannot univocally measure the reactor angle $\theta_{13}$ in presence of quasi-Dirac neutrinos. However, it is still possible to simultaneously constrain several of these angles. If not, Daya Bay would have observed a different signal. This penalty can be obtained from Eq. (7.15) by imposing $\left(1-X_{1}-X_{2}\right) X_{1}+X_{1} X_{2}=\sin ^{2} \theta_{\mathrm{DB}}$ where $\sin ^{2} \theta_{\mathrm{DB}} \approx 0.022$ is the value currently measured by the Daya Bay reactor experiment [84]. Hence, our global $\chi^{2}$ function can be written as

$$
\chi^{2}(p)=\chi_{\mathrm{DUNE}}^{2}(p)+\chi_{\mathrm{JUNO}}^{2}(p)+f_{\mathrm{DB}}(p) .
$$

The penalty function in terms of the relevant mixing angles is given by

$$
f_{\mathrm{DB}}(p)=\left[\frac{\left(\left(c_{14} c_{15} c_{16} s_{13}\right)^{2}+s_{16}^{2}-1\right)\left(\left(c_{14} c_{15} c_{16} s_{13}\right)^{2}+s_{16}^{2}\right)-\sin ^{2} \theta_{\mathrm{DB}}}{\sigma_{\mathrm{DB}} \sin ^{2} \theta_{\mathrm{DB}}}\right]^{2},
$$

where $c_{i j}=\cos \theta_{i j}, s_{i j}=\sin \theta_{i j}$ and $\sigma_{\mathrm{DB}}$ is the expected uncertainty in the final measurement of the reactor mixing angle by Daya Bay, set to $3 \%$. This is a generalization of the standard reactor prior used in several studies on neutrino oscillations.

\subsection{Results and discussion}

In this section, we present the results of the statistical analysis performed in this work. Before discussing the results of the combined analysis of DUNE and JUNO, we discuss the results of the two experiments separately. Note, however, that we always add the penalty term in Eq. 7.20 to the $\chi^{2}$ function obtained from the sensitivity analysis of each experiment. In Fig. 7.3 we show the twodimensional allowed regions obtained by scanning over the parameters $\theta_{13}, \theta_{23}$, $\theta_{16}, \theta_{26}, \delta_{13}$ and $\delta_{16}$ in DUNE. The parameters not shown are marginalized over in each panel. The colored regions correspond to the 1 (cyan), 2 (blue), 3 (red) $\sigma$ confidence levels for 2 degrees of freedom. In the upper panels, we see that the two reactor angles, $\theta_{13}$ and $\theta_{16}$, and their corresponding phases, $\delta_{13}$ and $\delta_{16}$, behave in a very similar way. In principle, small values of the phases are allowed, although these require very small values for the associated mixing angles. From 

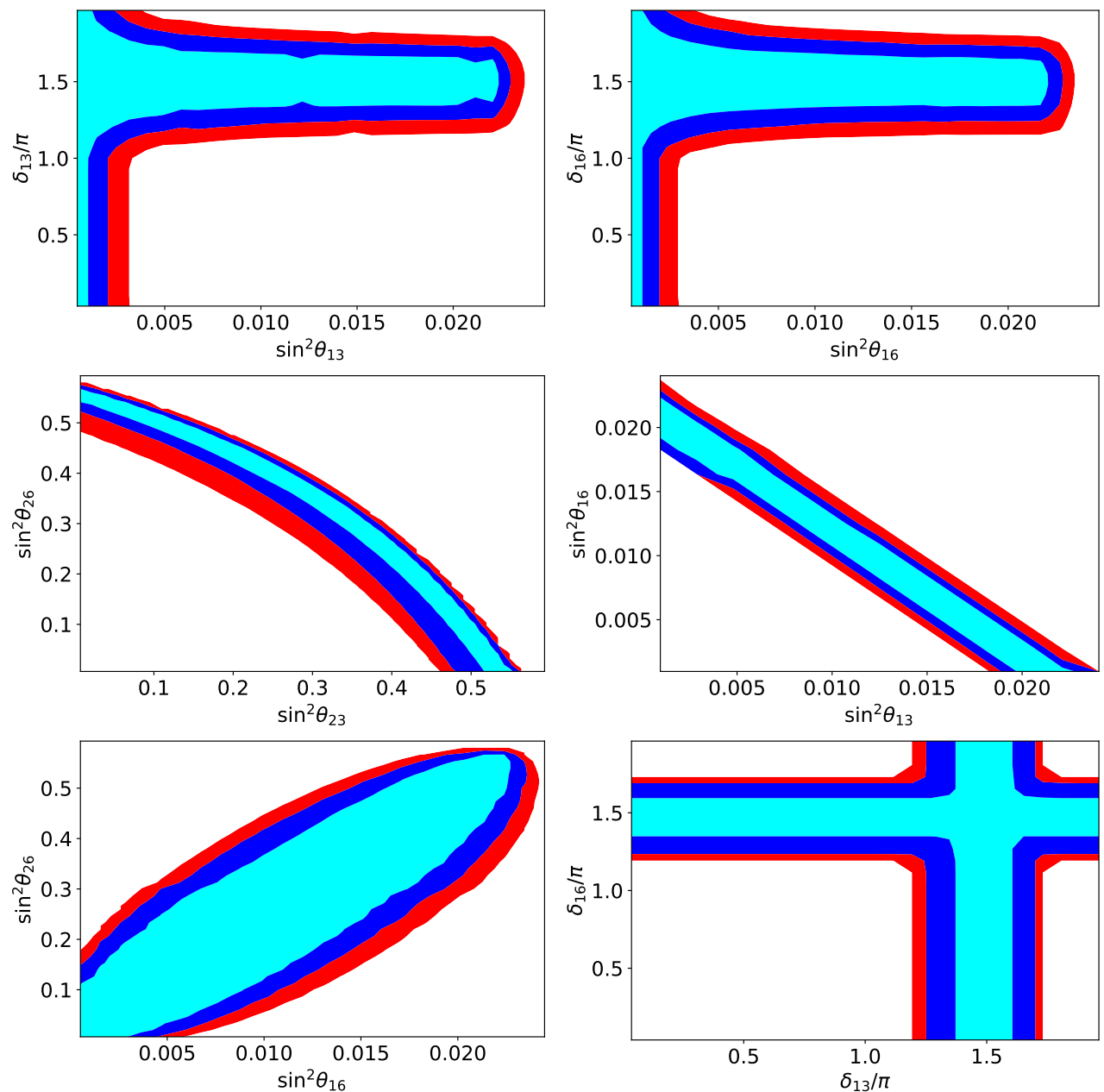

Figure 7.3: DUNE sensitivity to the oscillation parameters under study. The colored regions shown correspond to 1 (cyan), 2 (blue), 3 (red) $\sigma$ confidence levels for 2 degrees of freedom. In each two-dimensional plot we have marginalized over the other parameters which are not displayed. Figure taken from Ref. 15. 
the right panel of the second row, however, we see that both angles cannot be small at the same time: if $\theta_{13}$ is small, $\theta_{16}$ has to be large and vice versa. In the former case, $\delta_{13}$ can take any value in the interval $[0,2 \pi]$, while $\delta_{16}$ is rather restricted around its maximal value $1.5 \pi$. This is an interesting point, because the fake data were created with $\delta_{16}=0$. The reason behind this is that the new angles and phases are correlated to the standard angles, e.g. $\theta_{13}$ and $\theta_{16}$ (see the definitions in Eq. (7.14)), hence they are interchangeable. Note as well that all the sensitivity to the reactor angle is lost, since DUNE can only reproduce the prior [290] that we introduced as an input for our analysis, as explained in Sec. 7.3. The interchangeability of the mixing parameters can also be seen from the left panel of the second row in Fig. 7.3. There, we see that $\theta_{23}$ and $\theta_{26}$ are also fully correlated: having a large $\theta_{23}$ and a small $\theta_{26}$ is equivalent to having a small $\theta_{23}$ and a large $\theta_{26}$. The same applies to the CP-violating phases, as can be seen in the right panel of the last row. The left panel of the last row shows that there are correlations also between the atmospheric and reactor angles, which are not present in the standard case of three-neutrino oscillations anymore, given the very good level of precision achieved in the determination of the mixing angles. Since $\theta_{16}$ and $\theta_{13}$, as well as $\theta_{26}$ and $\theta_{23}$, are equivalent, a similar result is obtained in the two-dimensional plane $\left(\theta_{13}, \theta_{23}\right)$.

In Fig. 7.4 we show the result of our simulation of JUNO. In this case, we find that the new angles $\theta_{14}$ and $\theta_{15}$ are highly correlated with the standard solar angle $\theta_{12}$. In particular, one sees that, for $\sin ^{2} \theta_{14}=0$, all values of $\theta_{12}$ and $\theta_{15}$ lying along the correspondingly labeled line are possible, showing a similar correlation as in the case of $\theta_{13}$ and $\theta_{16}$ or $\theta_{23}$ and $\theta_{26}$. For different values of $\sin ^{2} \theta_{14}$ the correlating line is shifted as indicated in the figure. If we now marginalize over all possible values for $\theta_{14}$, we find that a large region of parameter space is still allowed. It is however important to notice that a point in the $\left(\sin ^{2} \theta_{12}, \sin ^{2} \theta_{15}\right)$ plane always corresponds to one specific value of $\sin ^{2} \theta_{14}$.

Now let us discuss the results of the combined analysis of DUNE and JUNO in terms of the variables $X_{i}$ introduced in Eq. (7.14). Compared to Ref. 290, where most of the parameter space was allowed by current neutrino oscillation data, here we find that DUNE and JUNO will be able to strongly constrain some of the $X_{i}$ parameters. This is illustrated in Fig. 7.5, where we show the $\Delta \chi^{2}$ profiles for the $X_{i}$ variables. In the left panel, we see how precise DUNE and JUNO could measure some of these quantities. Notably, $X_{1}, X_{2}$ and $X_{3}$ can be measured with a precision below $\%$. The sensitivity to $X_{4}, X_{5}, X_{6}$ and $X_{7}$ will also be improved with respect to the current results obtained in [290], although not 


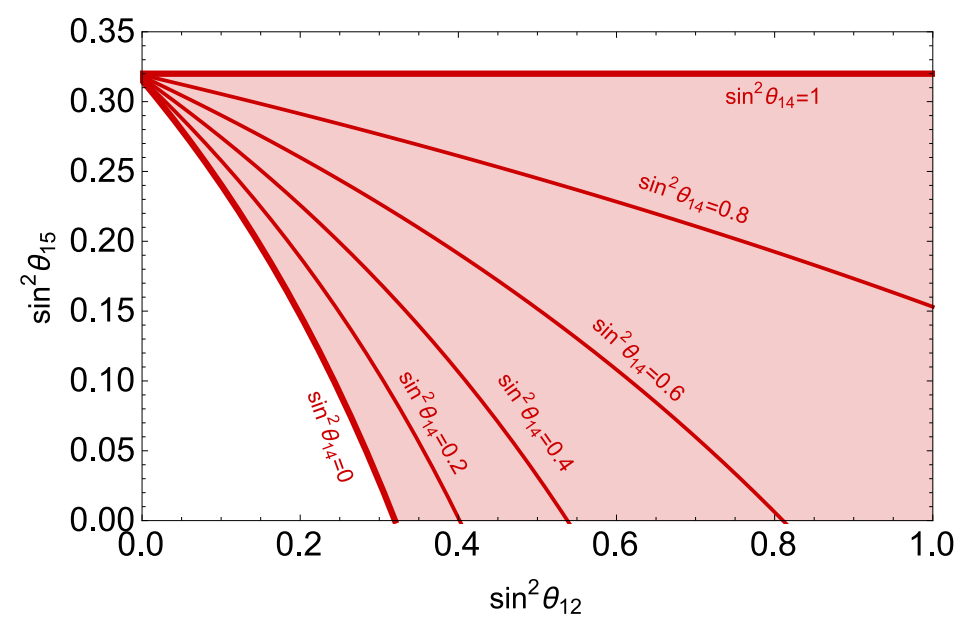

Figure 7.4: Sensitivity region in the $\left(\sin ^{2} \theta_{12}, \sin ^{2} \theta_{15}\right)$ plane for the JUNO experiment. The shaded region corresponds to the $1 \sigma$ allowed region once marginalized over $\sin ^{2} \theta_{14}$. See the text for more details. Figure taken from Ref. [15].

as dramatically as for the previous three parameters. Note, however, that DUNE will not be able to set a lower limit on $X_{4}, X_{6}$ and $X_{7}$, which are allowed to be zero in our combined fits. In the case of $X_{4}$, this can be traced to the fact that DUNE does not have the resolution to demonstrate that there are three independent oscillation frequencies contributing to $P\left(\nu_{\mu} \rightarrow \nu_{\mu}\right)$ (see Eq. (7.16)). An upper limit on $X_{4}$ can instead be obtained from the unitarity relation $X_{3}+X_{4}<1$. Similar comments apply to $X_{6}$ and $X_{7}$.

In the right panel of Fig. 7.5, we construct a quantity to test directly the Diracness of neutrino oscillations. This quantity is obtained by assuming that neutrinos are Dirac particles in Eq. (7.14), see Ref. 290 for more details. In this case one can derive that

$$
1-\frac{X_{5}}{X_{1} X_{3}}=0
$$

Any deviation from zero in this expression would be an indication for quasiDirac neutrinos. Since we created our fake data assuming neutrinos to be Dirac particles, our best-fit point is automatically located at zero. However, DUNE could restrict the allowed deviation considerably, as shown in the plot.

Finally, to further investigate the discrimination power of the experiments to the quasi-Dirac scenario, we have created another fake data set using a quasi- 

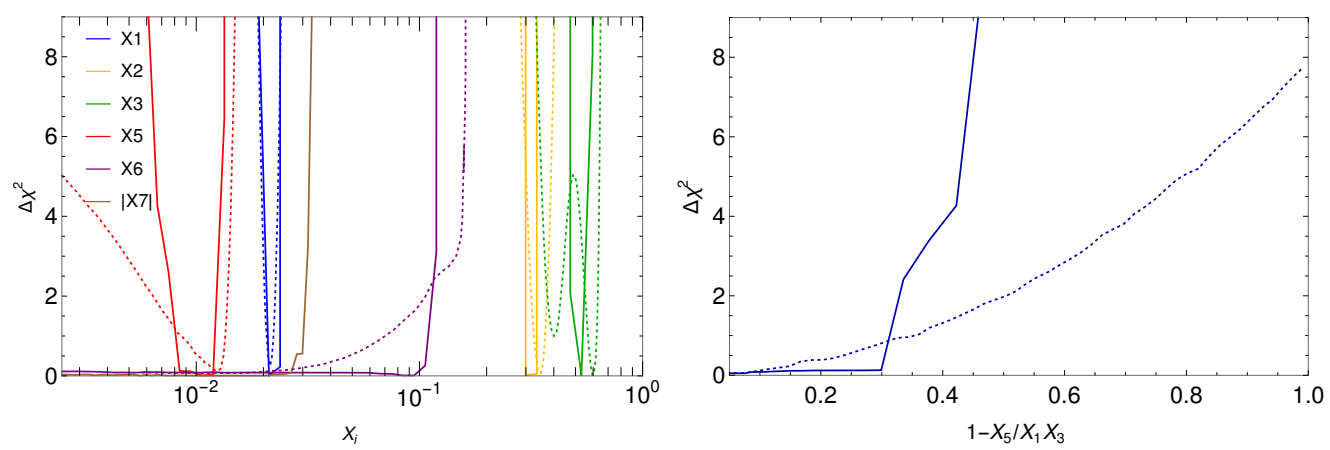

Figure 7.5: Left panel: $\chi^{2}$ profiles for the $X_{i}$ variables obtained in this analysis (solid lines) compared to the current bounds from Ref. 290 (dashed lines). Right panel: Diracness test for a Dirac input point obtained in this analysis (solid line) compared to the current bound from Ref. [290] (dashed line). Figure taken from Ref. 15 .

Dirac point as an input. For the particular point we have chosen, we expect

$$
1-\frac{X_{5}}{X_{1} X_{3}}=0.5
$$

Our choice falls inside the $1 \sigma$ contours of Fig. 7.3 and it corresponds to $\sin ^{2} \theta_{23}=0.30, \sin ^{2} \theta_{26}=0.37, \sin ^{2} \theta_{13}=0.0108, \sin ^{2} \theta_{16}=0.0108$. The CPviolating phases are assumed as in the first analysis. The result for this simulation is shown in Fig. 7.6. In the left panel one can see how most of the $X_{i}$ are mostly unaffected by the selected input point, while there is a visible difference in the profiles corresponding to $X_{3}$ and $X_{5}$. Nevertheless, the most visible effect appears in the right panel of the figure. There, we see that the Dirac-point with $1-\frac{X_{5}}{X_{1} X_{3}}=0$ - could be completely excluded in this scenario. This is an important result, because it means that DUNE and JUNO would be able to distinguish standard three-neutrino oscillations from quasi-Dirac oscillations. Note, however, that this statement is true for our benchmark point. If the true value lies very close to Diracness, it would be more difficult to discriminate between the two scenarios.

Let us mention that many of the degeneracies observed in the analysis could be broken by including a $\nu_{\tau}$ appearance channel in the DUNE analysis. This possibility has been recently discussed in Ref. 291,292. If neutrinos are quasi-Dirac particles, $\sum_{\beta} P_{\alpha \beta}<1$, with $\beta=\{e, \mu, \tau\}$. Hence, a more precise observation of 



Figure 7.6: Left panel: $\chi^{2}$ profiles for the $X_{i}$ variables. Right panel: Diracness test for a quasi-Dirac input point. Figure taken from Ref. [15.

the unitarity of neutrino oscillations including the $\nu_{\tau}$ channel would be extremely helpful to test the quasi-Dirac neutrino hypothesis, as well as other non-unitary neutrino scenarios 293,294]. 



\section{Chapter 8}

\section{Long live the neutrino}

The last new physics scenario we study in this thesis is the possibility of unstable neutrinos. If neutrinos decay, this decay should alter the expected event rates for a given experiment. In this chapter, we analyze the effect a decaying neutrino could have on the ORCA experiment, turning our attention from long baseline accelerators to atmospheric neutrino oscillation experiments. The results of this chapter are published in Ref. [16].

\subsection{Introduction}

The determination of the mass ordering of neutrinos is one of the main physics goals of the future atmospheric experiment ORCA $[152$, that will provide precise measurements of the atmospheric parameters too, see chapter 4 . ORCA seems a very promising candidate not only to improve the current sensitivity to neutrino oscillation parameters, but also to look for signals of physics beyond the Standard Model. The presence of new physics might change the well established picture of neutrino oscillations and, hence, it is crucial to improve the precision of current measurements to look for potential deviations of the standard scenario, which would only arise at sub-leading order.

One of these new physics scenarios, able to alter the neutrino oscillation pattern, is based on the existence of unstable neutrinos. Taking, for example, the Majoron model 295 299], a neutrino $\nu_{i}$ can decay into a lighter neutrino $\nu_{j}$ and a new boson, the Majoron $J$, through $\nu_{i} \rightarrow \nu_{j}+J$ or $\nu_{i} \rightarrow \bar{\nu}_{j}+J$. Likewise, Dirac neutrinos could decay through $\nu_{i L} \rightarrow \nu_{j R}+\xi$ into a scalar $\xi$ and a light right-handed neutrino $\nu_{j}$. If the decay product $\nu_{j}$ is an active neutrino, we talk about a visible neutrino decay, otherwise it is an invisible decay. Here we focus 
on the latter one. For different studies on visible neutrino decay at current or future experiments, we refer to Refs. [300 306].

Actually, the idea of unstable neutrinos is not new, since it was already proposed to explain the solar neutrino anomaly with a decaying mass state $\nu_{2}$ [307]. However, at some point, neutrino decay alone could not explain the solar neutrino deficit anymore and flavor oscillations were needed anyway 308. Therefore, it is usually assumed that this process can appear at subdominant level in combination with neutrino oscillations, as studied in Refs. 309 312. For the invisible neutrino decay, the best bound from oscillation experiments on the $\nu_{2}$ lifetime comes from the combination of solar and reactor data and corresponds to approximately $\tau_{2} / m_{2}>2 \times 10^{-3} \mathrm{~s} / \mathrm{eV}$ at $90 \%$ C.L. 313. See also Ref. 314 for a similar result and Ref. [315] for a recent forecast analysis on these parameters using dark matter detectors.

The decay of the third neutrino mass eigenstate, $\nu_{3}$, was also considered as an attempt to explain the atmospheric neutrino problem, but it was found that also here it can contribute only with sub-leading effects [316]. Several studies using atmospheric and long-baseline experiments have been performed in this direction. For instance, the analysis in Ref. 317] combines neutrino data from SuperKamiokande, K2K and MINOS to obtain the limit $\tau_{3} / m_{3}>2.9 \times 10^{-10} \mathrm{~s} / \mathrm{eV}$ at $90 \%$ C.L. The authors of Ref. [318], on the other hand, combine long-baseline neutrino data from T2K and MINOS, obtaining the bound $\tau_{3} / m_{3}>2.8 \times 10^{-12}$ $\mathrm{s} / \mathrm{eV}$ at $90 \%$ C.L. Note, however, that these results have been derived under the two-neutrino approximation and, therefore, a full three-neutrino analysis might loosen this bound. Recently, following a three-neutrino approach, a new constraint on the neutrino decay lifetime has been calculated from the combination of $\mathrm{T} 2 \mathrm{~K}$ and $\mathrm{NO} \nu \mathrm{A}$ data $[319]$, giving approximately $\tau_{3} / m_{3}>2 \times 10^{-12} \mathrm{~s} / \mathrm{eV}$ at 90\% C.L.

Prompted by the good sensitivity of the forthcoming experiment KM3NeTORCA to the atmospheric neutrino oscillation parameters in the $\mathrm{GeV}$ energy range, it is worth studying whether it can provide better bounds on the invisible neutrino decay, in comparison to other neutrino oscillation experiments.

\subsection{Invisible neutrino decay}

Here we discuss how the presence of an invisible neutrino decay would affect the calculation of the neutrino oscillation probability. Besides the three light known neutrinos, we consider the presence of a fourth sterile neutrino, $\nu_{4}$. Along this work, we will assume the decay of the heaviest mass eigenstate $\left(\nu_{3}\right.$ in NO) 
to this new neutrino state

$$
\nu_{3} \rightarrow \nu_{4}+J
$$

where $J$ is a pseudo-scalar singlet, or Majoron. We assume that there is no mixing among the three active neutrinos and the sterile one, so it can not oscillate back into an active state. Therefore, the neutrino mixing matrix in vacuum is given by the standard three-family mixing matrix $U$,

$$
\left(\begin{array}{l}
\nu_{\alpha} \\
\nu_{s}
\end{array}\right)=\left(\begin{array}{ll}
U & 0 \\
0 & 1
\end{array}\right)\left(\begin{array}{l}
\nu_{k} \\
\nu_{4}
\end{array}\right)
$$

where the Greek index $\alpha=e, \mu, \tau$ indicates the flavor eigenstates and the Latin index $k=1,2,3$, the mass eigenstates. Because of the absence of active-sterile mixing, the propagation of the active states is not affected by the presence of $\nu_{4}$. For the mass spectrum we assume normal mass ordering for the active states and a fourth state $\nu_{4}=\nu_{s}$, lighter than the decaying one $m_{4}<m_{3}$. To take into account the neutrino decay in the evolution process, we modify the neutrino Hamiltonian, including a decay constant $\alpha_{3}=m_{3} / \tau_{3}$, where $m_{3}$ is the heaviest neutrino mass and $\tau_{3}$ is its rest-frame lifetime. Hence, the full neutrino Hamiltonian can be written as

$$
H=\frac{1}{2 E}\left[H_{0}+H_{m}+H_{D}\right]
$$

where the first two terms correspond to the standard vacuum and matter terms, namely

$$
H_{0}=U\left(\begin{array}{ccc}
0 & 0 & 0 \\
0 & \Delta m_{21}^{2} & 0 \\
0 & 0 & \Delta m_{31}^{2}
\end{array}\right) U^{\dagger}, \quad H_{m}=\left(\begin{array}{ccc}
V & 0 & 0 \\
0 & 0 & 0 \\
0 & 0 & 0
\end{array}\right)
$$

with $V=2 E \sqrt{2} G_{F} N_{e} . E$ is the neutrino energy, $G_{F}$ the Fermi constant and $N_{e}$ the electron number density. Finally, the last term in Eq. 8.3 represents the neutrino decay part

$$
H_{D}=U\left(\begin{array}{ccc}
0 & 0 & 0 \\
0 & 0 & 0 \\
0 & 0 & -i \alpha_{3}
\end{array}\right) U^{\dagger}
$$

Then, effectively, the only change to the standard oscillation picture is a shift in the 33 entry of the Hamiltonian in the mass basis, from $\Delta m_{31}^{2}$ to $\Delta m_{31}^{2}$ $i \alpha_{3}$. Note, however, that this implies that the sum of the neutrino oscillation 
probabilities might be different from one,

$$
P_{\alpha e}+P_{\alpha \mu}+P_{\alpha \tau}<1, \quad \alpha=e, \mu, \tau .
$$

Therefore, if the heaviest neutrino mass eigenstate $\nu_{3}$ decays, apart from a changed oscillatory pattern, we could also have missing neutrinos. In order to show the effect of the decay, we present in Fig. 8.1 the difference in the survival probability (left panel) of atmospheric muon neutrinos with and without decay, $\Delta P_{\alpha \beta}=P_{\alpha \beta}^{\text {decay }}-P_{\alpha \beta}^{\text {standard }}$, for a value of $\alpha_{3}=10^{-5} \mathrm{eV}^{2}$. The right panel shows the analogous result for the electron neutrino appearance probability. Note that this value of the decay constant is rather large and we have chosen it for illustrative purposes only. As it is seen in the figures, the main effect of the decay is concentrated in the region of neutrino energies close to the resonance $(\sim 3-8 \mathrm{GeV})$ for values of the zenith angle at which matter effects are more relevant. This results in a softening of the oscillation pattern, as better illustrated in Fig. 8.2 . There, we show the muon neutrino survival probability $P_{\mu \mu}$ as well as the electron neutrino appearance probability $P_{\mu e}$ for a particular arrival direction, corresponding to $\cos \theta_{Z}=-0.82$. The solid lines in the plot correspond to the standard case, without neutrino decay, while the dashed, dashed-dotted and dotted lines have been obtained assuming values of the decay constant $\alpha_{3}$ equal to $10^{-5}, 10^{-4}$ and $10^{-3} \mathrm{eV}^{2}$, respectively. In this figure, one can see how the presence of the invisible neutrino decay leads to a reduction of the oscillatory behavior, that becomes almost suppressed for larger values of $\alpha_{3}$, with special impact for the values of $\cos \theta_{Z}$ and neutrino energies close to the matter effect resonance at around $6-7 \mathrm{GeV}$. An interesting result from this suppression is the increment of the survival probability at some regions in the plane $\cos \theta_{Z}-E$, which seems contradictory with the idea of decaying neutrinos. This comes from the softening of flavor oscillations in the presence of the $\nu_{3}$ decay, since it washes out oscillations controlled by $\Delta m_{31}^{2}$ while keeping untouched those driven by $\Delta m_{21}^{2}$. As a consequence, an almost averaged oscillation pattern appears, which enhances the survival muon neutrino probability at its minima. However, Eq. 8.6 holds and the sum of neutrino probabilities below unity tells us that they are actually decaying.

\subsection{Numerical analysis}

In this section, we present the numerical procedure followed to simulate the neutrino signal in ORCA. First, we explain how to calculate the number of events 

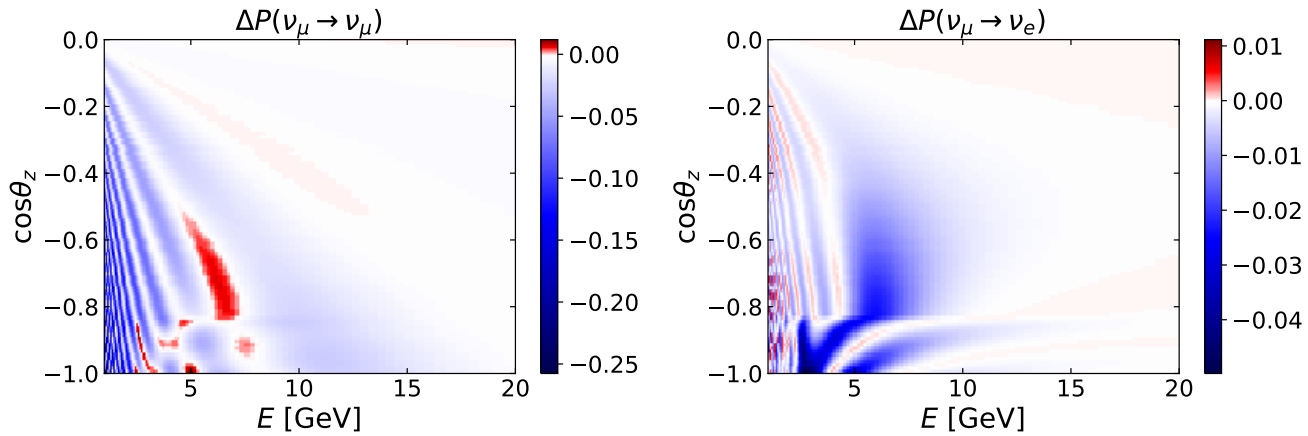

Figure 8.1: Differences in the oscillation probability of atmospheric muon neutrinos with and without decay, $\Delta P=P^{\text {decay }}-P^{\text {standard }}$, for the $\nu_{\mu} \rightarrow \nu_{\mu}$ (left panel) and $\nu_{\mu} \rightarrow \nu_{e}$ (right panel) oscillation channels. A value of $\alpha_{3}=10^{-5} \mathrm{eV}^{2}$ has been assumed in both cases. The difference in the probabilities is shown as a function of the neutrino energy and arrival zenith angle. Figure taken from Ref. [16].

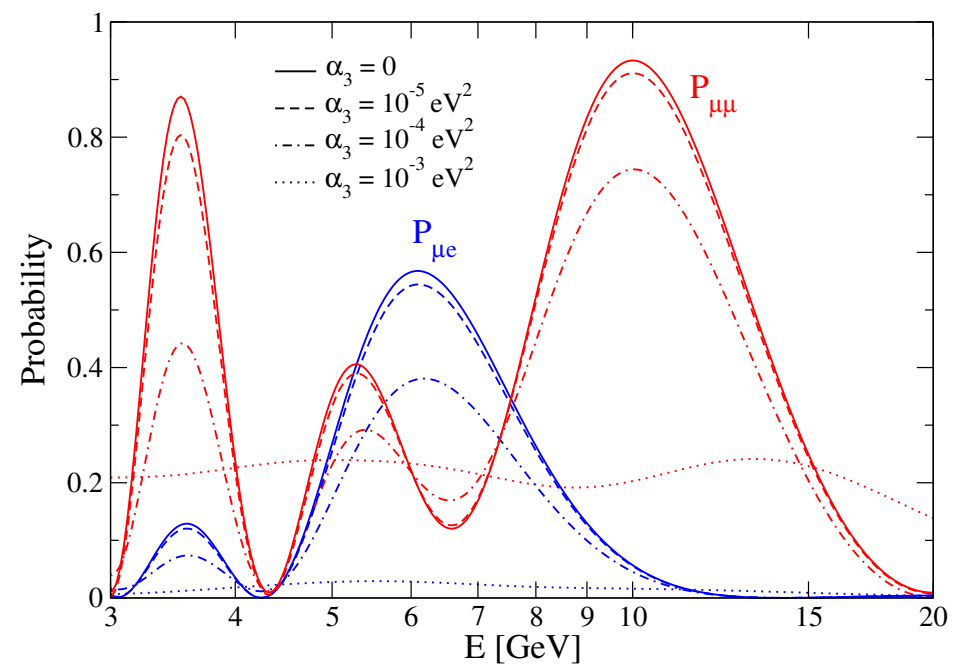

Figure 8.2: Muon neutrino survival probability, $P_{\mu \mu}$, and electron neutrino appearance probability, $P_{\mu e}$, as a function of the energy for the arrival direction $\cos \theta_{Z}=-0.82$. The solid lines correspond to the standard case, without neutrino decay, while the dashed, dashed-dotted and dotted lines have been obtained assuming $\alpha_{3}=10^{-5}, 10^{-4}$ and $10^{-3} \mathrm{eV}^{2}$, respectively. Figure taken from Ref. 16. 
and the $\chi^{2}$ functions. Next, we provide some details about how to handle the systematic uncertainties in the data analysis.

\subsubsection{Simulation of the neutrino signal in ORCA}

The simulation of the neutrino signal expected in ORCA requires the knowledge of the atmospheric neutrino flux, that we take from [98] and further modulate with flavor oscillations. The conversion probability, from the neutrino creation point in the atmosphere to the detector after traversing the Earth, is numerically calculated considering three-neutrino oscillations in matter. In order to do so, we discretize the neutrino path and consider the matter density at each point using the PREM profile [100].

The sensitivity tests presented in this work are performed simulating the number of events detected by ORCA in a binned area of the parameters $\cos \theta_{z \text {,rec }}$ and $\log _{10}\left(E_{\nu, \text { rec }} / \mathrm{GeV}\right)$, where $\theta_{z \text {,rec }}$ is the reconstructed zenith angl $\oint^{1}$ and $E_{\nu \text {,rec }}$ is the reconstructed neutrino energy. Following the indications in Ref. [152, we divide the reconstructed parameter ranges, $\cos \theta_{z \text {,rec }} \in[-1,0]$ and $\varepsilon_{\text {rec }}=$ $\log _{10}\left(E_{\nu, \text { rec }} / \mathrm{GeV}\right)$, in 20 bins each, where $E_{\nu, \text { rec }} \in[1,21] \mathrm{GeV}$. We have also considered larger values for the maximum reconstructed neutrino energy, but the results are essentially unchanged.

Given the incapability of ORCA to distinguish neutrinos from antineutrinos, both contributions are summed in each bin. Our analysis, however, makes a distinction between the two different topologies produced in a neutrino interaction with the sea water molecules, namely track-like or shower-lik $2^{2}$ A track is an elongated signal of deposited energy in ORCA's photomultipliers (PMTs), which is mostly produced when a $\nu_{\mu}$ (or its antiparticle) interacts through chargedcurrent $(\mathrm{CC})$ interactions, producing a muon that travels a long distance before losing all its energy. The same topology can be produced in a $\nu_{\tau} \mathrm{CC}$ interaction if the generated tau decays into a muon, which produces the track. Therefore, this constitutes an unavoidable background for muon neutrinos detected through CC. On the other hand, in all other cases $\left(\nu_{e} \mathrm{CC}, \nu_{\tau} \mathrm{CC}\right.$ with the tau not decaying into a muon and all flavor neutral-current interactions) the neutrino energy is quickly lost into an electromagnetic cascade, a hadronic cascade or both, depending on the interaction, giving rise to a shower-like topology. In current large-volume neutrino telescopes, like ANTARES or IceCube, the directionality of an event

\footnotetext{
${ }^{1}$ The zenith angle is defined such that $\theta_{z}=0$ corresponds to vertical down-going events.

${ }^{2}$ Interestingly, invisible neutrino decay has been recently suggested as a way to explain the tension between the event topologies (tracks or cascades) of the detected high-energy astrophysical neutrinos at the IceCube observatory 320 .
} 
producing a shower is measured with a large uncertainty. However, thanks to the multi-PMT characteristic of ORCA's DOMs, the directionality of a shower-like event will be known with a precision of a few degrees, where the exact accuracy depends on the incoming neutrino energy (less than 10 degrees for energies larger than $5 \mathrm{GeV}) 152$.

The expected number of events per bin, $N_{i j}^{c \alpha}$, corresponding to a given interaction channel, $c$, and a neutrino flavor, $\alpha$, contributing to a given topology, is calculated from the convolution of the neutrino flux at the detector with the corresponding cross section, detector resolutions and detector effective mass,

$$
\begin{aligned}
N_{i j}^{c \alpha} & =2 \pi t \ln ^{2}(10) \int_{\cos \theta_{z, \text { rec }}^{i}}^{\cos \theta_{z, \text { rec }}^{i+1}} \mathrm{~d} x \int_{\varepsilon_{\text {rec }}^{j}}^{\varepsilon_{\text {rec }}^{j+1}} \mathrm{~d} y \int_{-1}^{1} \mathrm{~d} \cos \theta_{z} \\
& \times \int_{-\infty}^{+\infty} \mathrm{d} \varepsilon_{\text {true }} E_{\text {rec }}(y) E_{\text {true }}\left(\varepsilon_{\text {true }}\right) \frac{M_{\text {eff }}^{c \alpha}\left(\varepsilon_{\text {true }}\right)}{m_{p}} \\
& \times R^{c \alpha}\left(x, y, \cos \theta_{z}, \varepsilon_{\text {true }}\right) \frac{\mathrm{d}^{2} \phi_{\text {det }}^{c \alpha}}{\mathrm{d} \cos \theta_{z} \mathrm{~d} E_{\text {true }}}\left(\cos \theta_{z}, \varepsilon_{\text {true }}\right),
\end{aligned}
$$

where $t$ is the total time of data acquisition, $x$ and $y$ are abreviations for the reconstructed zenith angle and the logarithm of the reconstructed energy, respectively, $m_{p}$ the proton mass, $\varepsilon_{\text {true }}=\log _{10}\left(E_{\nu, \text { true }} / \mathrm{GeV}\right) . M_{\text {eff }}^{c \alpha}, R^{c \alpha}$ and $\phi_{\text {det }}^{c \alpha}$ are the detector effective mass, the detector resolution and the number of neutrinos per second at the detector for the corresponding interaction channel and neutrino flavor, respectively. The indices $i$ and $j$ refer to the $i$ th bin in reconstructed zenith angle $\theta_{\mathrm{z} \text {,rec }}$ and the $j$ th bin in reconstructed energy $\varepsilon_{\text {rec }}$. The number of $\nu_{\alpha}$ per second at the detector for the interaction channel $c$ is given by

$$
\frac{\mathrm{d}^{2} \phi_{\text {det }}^{c \alpha}}{\mathrm{d} \cos \theta_{z} \mathrm{~d} E_{\text {true }}}=\sigma^{c \alpha} \sum_{\beta=\{e, \mu\}} \frac{\mathrm{d}^{2} \phi_{\beta}^{0}}{\mathrm{~d} \cos \theta_{z} \mathrm{~d} E_{\text {true }}} P_{\nu_{\beta} \rightarrow \nu_{\alpha}},
$$

where $\sigma^{c \alpha}$ is the cross section for $\nu_{\alpha}$ in the interaction channel $c$ 321, 322, $\phi_{\beta}^{0}$ is the atmospheric neutrino flux $[98]$ and $P_{\nu_{\beta} \rightarrow \nu_{\alpha}}$ is the probability for a $\nu_{\beta}$ to oscillate into a $\nu_{\alpha}$ when arriving at ORCA. To simulate the atmospheric neutrino signal in ORCA, we have fixed the values of the neutrino oscillation parameters to their best-fit values found in chapter 2 and summarized in Tab. 8.1. Note that, to establish ORCA's sensitivity to the invisible neutrino decay, we set the decay parameter $\alpha_{3}$ to zero in the simulated data. 


\begin{tabular}{|c|c|}
\hline parameter & value \\
\hline$\Delta m_{21}^{2}$ & $7.55 \times 10^{-5} \mathrm{eV}^{2}$ \\
$\Delta m_{31}^{2}$ & $2.50 \times 10^{-3} \mathrm{eV}^{2}$ \\
$\sin ^{2} \theta_{12}$ & 0.32 \\
$\sin ^{2} \theta_{23}$ & 0.547 \\
$\sin ^{2} \theta_{13}$ & 0.0216 \\
$\delta$ & $1.32 \pi$ \\
\hline$\alpha_{3}$ & 0 \\
\hline
\end{tabular}

Table 8.1: Neutrino oscillation parameters and decay constant used to simulate the atmospheric data in ORCA.

The detector resolutions in Eq. (8.7), $R^{c \alpha}$, include both the zenith angle and energy resolutions

$$
R^{c \alpha}\left(\theta_{z, \text { rec }}, E_{\nu, \text { rec }}, \theta_{z, \text { true }}, E_{\nu, \text { true }}\right)=r_{E_{\nu}}^{c \alpha}\left(E_{\nu, \text { rec }}, E_{\nu, \text { true }}\right) r_{\theta_{z}}^{c \alpha}\left(\theta_{z, \text { rec }}, \theta_{z, \text { true }}, E_{\nu, \text { true }}\right),
$$

where the dimensions of the individual resolutions are given by $\left[r_{E_{\nu}}^{c \alpha}\right]=\mathrm{GeV}^{-1}$ and $\left[r_{\theta_{z}}^{c \alpha}\right]=\operatorname{rad}^{-1}$. For simplicity, here we have neglected the dependence of the energy resolution on the arrival direction, that is actually very small $[152$.

Finally, with all these ingredients, one can calculate the total number of events for a given topology (track-like or shower-like) expected in ORCA. The total event number is obtained by convolving each interaction channel with the corresponding particle identification performance: $T_{\text {pid }}^{c \alpha}$ for tracks and $S_{\text {pid }}^{c \alpha}$ for showers. Each of them represents the probability that ORCA identifies an event produced from a $\nu_{\alpha}$ via an interaction channel $c$ with the given topology. Thus, the number of events inside the $i j$-bin identified with a topology $\mathcal{T}$ is

$$
N_{i j}^{\mathcal{T}}=\sum_{\alpha=\{e, \mu, \tau\}} \sum_{c} \mathcal{T}_{\mathrm{pid}}^{c \alpha} N_{i j}^{c \alpha}
$$

with $\mathcal{T}_{\text {pid }}^{c \alpha}=T_{\text {pid }}^{c \alpha}, S_{\text {pid }}^{c \alpha}$. The detector-dependent quantities $\left(M_{\text {eff }}^{c \alpha}, R^{c \alpha}\right.$ and $\left.\mathcal{T}_{\text {pid }}^{c \alpha}\right)$ corresponding to ORCA have been obtained by fitting the information provided in 152 for a configuration of $9 \mathrm{~m}$ spacing between DOMs in a line, chosen as the final experimental setup by the KM3NeT collaboration. 


\subsubsection{The role of systematic uncertainties}

We estimate ORCA's sensitivity to the invisible neutrino decay defining a $\chi^{2}$ function in terms of the most relevant parameters: the decay constant $\alpha_{3}$ and the atmospheric parameters, $\Delta m_{31}^{2}$ and $\sin ^{2} \theta_{23}$. With this $\chi^{2}$, we fit the simulated signal in ORCA, obtained as explained in the previous subsection. Besides the details commented there, one should also consider the presence of systematic uncertainties that may affect the simulation of the experiment. For instance, the uncertainties on the determination of the atmospheric neutrino flux or the limited knowledge of the detector response will certainly modify the calculation of the expected number of events in a given experiment. These systematic uncertainties are usually included in the numerical analysis with some nuisance parameters $\epsilon_{i}$, implemented in the definition of the $\chi^{2}$ function

$$
\begin{aligned}
\chi^{2}\left(\sin ^{2} \theta_{23}, \Delta m_{31}^{2}, \alpha_{3}\right) & =\min _{\vec{\epsilon}}\left\{\sum_{i, j}\left(\frac{N_{i j}\left(\sin ^{2} \theta_{23}, \Delta m_{31}^{2}, \alpha_{3} ; \vec{\epsilon}\right)-N_{i j}^{\mathrm{dat}}}{\sqrt{N_{i j}^{\mathrm{dat}}}}\right)^{2}\right. \\
& \left.+\sum_{k}\left(\frac{\epsilon_{k}-\mu_{k}}{\sigma_{k}}\right)^{2}\right\} .
\end{aligned}
$$

Here $i(j)$ indicates the $i$ th $\left(j\right.$ th) bin in azimuth angle (energy), $N_{i j}^{\text {dat }}$ is the simulated number of events in this bin (in analogy with the observed one for a running experiment) and $N_{i j}\left(\sin ^{2} \theta_{23}, \Delta m_{31}^{2}, \alpha_{3} ; \vec{\epsilon}\right)$ is the event number for the oscillation parameters $\sin ^{2} \theta_{23}$ and $\Delta m_{31}^{2}$, the decay constant $\alpha_{3}$ and the nuisance parameters $\vec{\epsilon}=\left(\epsilon_{1}, \epsilon_{2}, \ldots\right)$. These parameters are fitted after the minimization of the $\chi^{2}$ function, that includes a pull factor penalizing large deviations from their corresponding expectation values, $\mu_{k}$, compared to the associated errors, $\sigma_{k}$. The systematic uncertainties used in our analysis are listed in Tab. 8.2, together with their corresponding expectation values and errors. As explained above, we consider systematic uncertainties related to the detector functionality and to the theoretical predictions of the atmospheric neutrino fluxes. The first four entries in Tab. 8.2 are related to the atmospheric neutrino flux as, for instance, a global normalization uncertainty, $\mathcal{N}$. We also consider $\gamma$, the deviation of the spectral index (with a pivot point in neutrino energy at $20 \mathrm{GeV}$ ), and two systematic uncertainties related to the composition of the atmospheric flux, namely the fraction of muon neutrinos to electron neutrinos $\phi_{\nu_{\mu}} / \phi_{\nu_{e}}$ in the original fluxes: $f_{\mu e}$, and another one regarding the fraction of neutrinos to antineutrinos $\phi_{\nu} / \phi_{\bar{\nu}}: f_{\nu / \bar{\nu}}$. These last two modify the flux composition, such that $\phi \rightarrow \tilde{\phi}$. In combination 


\begin{tabular}{|c|c|c|}
\hline Systematic & $\begin{array}{c}\text { Expectation } \\
\text { value }\left(\mu_{k}\right)\end{array}$ & $\begin{array}{c}\text { Standard } \\
\text { deviation }\left(\sigma_{k}\right)\end{array}$ \\
\hline $\mathcal{N}$ & 1 & flat \\
$\gamma$ & 0 & flat \\
$f_{\mu e}$ & 0 & 0.1 \\
$f_{\nu / \bar{\nu}}$ & 0 & 0.1 \\
\hline$f_{r, \text { shower }}$ & 0 & 0.2 \\
$f_{r, \text { track }}$ & 0 & 0.2 \\
$f_{E}$ & 0 & 0.01 \\
\hline
\end{tabular}

Table 8.2: List of systematic uncertainties used to reproduce ORCA's functionality and uncertainties in the atmospheric neutrino fluxes, where $\mu_{k}$ and $\sigma_{k}$ are the corresponding central value and Gaussian dispersion, respectively.

with the other systematic uncertainties, the flux is changed as

$$
\phi\left(E, \theta_{z}\right) \rightarrow \mathcal{N} \tilde{\phi}\left(E, \theta_{z}\right)\left(\frac{E}{E_{\text {pivot }}}\right)^{\gamma} .
$$

The rest of the systematic uncertainties $\left(f_{r \text {,shower }}, f_{r \text {,track }}\right.$ and $\left.f_{E}\right)$ are associated to detector-related effects that might affect, respectively, the resolution of shower and track events, as well as the reconstructed energy. The first two simply modify the detector response function in Eq. (8.9), discussed in the last subsection, while $f_{E}$ modifies the incoming (or true) neutrino energy. This systematic uncertainty affects the event number calculation in a more complicated way, since it replaces $E_{\text {true }}$ by $E_{\text {true }}\left(1+f_{E}\right)$ everywhere in the simulation and, therefore, modifies directly the oscillation probabilities. Note that all the systematic uncertainties named with an $f$ account for small deviations from their corresponding central value. Therefore, their effect can be accounted for by modifying the corresponding variable $X$ to which they affect, such that $X \rightarrow X\left(1+f_{X}\right)$.

\subsection{Results and discussion}

In this section, we present the results of our analysis, assuming that the ORCA experiment is running for three or ten years. First, we present the bounds it could put on the invisible decay of $\nu_{3}$ from the observation of atmospheric neutrino oscillations. Next, we show how the invisible neutrino decay can affect the determination of the atmospheric neutrino parameters when marginalizing 
over $\alpha_{3}$. Since current global data prefer normal neutrino mass ordering [3, 4], here we will focus only on this case.

\subsubsection{Estimated sensitivity to the invisible decay}

Our results for the sensitivity to the decay constant $\alpha_{3}$ are presented in Fig. 8.3. We have followed two approaches to estimate ORCA's sensitivity to the invisible decay. First, we have fixed the oscillation parameters, $\Delta m_{31}^{2}$ and $\sin ^{2} \theta_{23}$, to their best fit point and we have calculated the $\chi^{2}$ function following Eq. 8.11). The results of this analysis are reported with dashed lines in Fig. 8.3, where we have considered 3 and 10 years of running time. Then, we have followed a more realistic procedure, minimizing the $\chi^{2}$ function defined in Eq. 8.11 over the oscillation parameters for each value of $\alpha_{3}$,

$$
\chi_{\text {decay }}^{2}\left(\alpha_{3}\right)=\min _{\sin ^{2} \theta_{23}, \Delta m_{31}^{2}}\left[\chi^{2}\left(\sin ^{2} \theta_{23}, \Delta m_{31}^{2}, \alpha_{3}\right)\right] .
$$

The minimization is performed over all possible values of $\Delta m_{31}^{2}$ and $\sin ^{2} \theta_{23}$, where no priors on the parameters have been used. Our findings for this case correspond to the solid lines plotted in Fig. 8.3. Thanks to the expected good resolution of ORCA to $\Delta m_{31}^{2}$ and $\theta_{23}$, the prior knowledge on the oscillation parameters affects the sensitivity to neutrino decay only for very large values of $\alpha_{3}$, as can be seen by comparing the solid and dashed lines in the two panels of Fig. 8.3. This worsening of the sensitivity comes mostly from a jump in the preferred value of $\theta_{23}$ from the second octant (corresponding to the best-fit value of $\theta_{23}$, used to simulate ORCA's data) to the first one. We have tested that the change of behavior in the sensitivity curves actually disappears when a value of $\sin ^{2} \theta_{23}<0.5$ is chosen to simulate the fake data. The reason of this feature is somewhat subtle, but it is related to the fact that larger modifications of the oscillation probabilities due to neutrino decay are expected close to the matter effect resonance, as explained in Sec. 8.2. In this region, there is a large influence coming from the neutrino appearance probabilities, $P_{\nu_{e, \mu} \rightarrow \nu_{\mu, e}}$, mainly driven by a term proportional to $\sin ^{2} \theta_{23}$ (contrary to the muon neutrino disappearance probability, dominated by $\left.\sin ^{2} 2 \theta_{23}\right) 152$. Because a larger decay essentially implies less track-like events $\left(\nu_{\mu}\right.$ contains more $\nu_{3}$ than $\nu_{e}$ does $)$, and the $\theta_{23}$ dependence makes the muon disappearance probability almost flat around $\sin ^{2} \theta_{23}=0.5$ when compared to the appearance probabilities, this reduction of track-like signatures due to neutrino decay can be compensated with a lower rate of flavor oscillations 

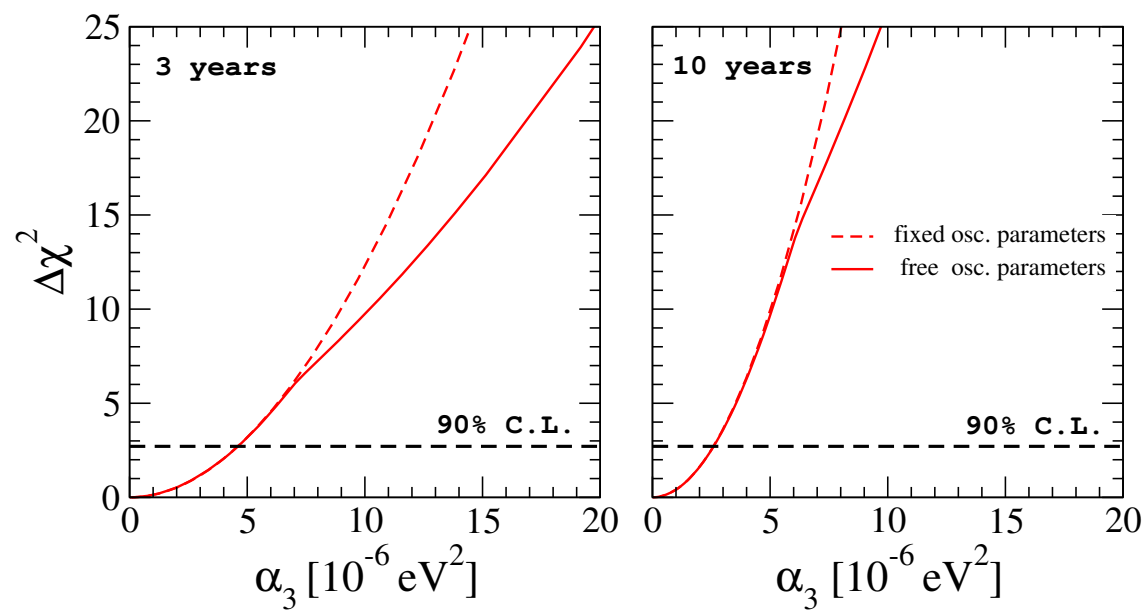

Figure 8.3: Sensitivity of ORCA to the neutrino decay constant $\alpha_{3}$. Solid (dashed) lines correspond to the analysis with free (fixed) oscillation parameters $\Delta m_{31}^{2}$ and $\theta_{23}$. The left (right) panel shows the results for three (ten) years of operational time. Figure taken from Ref. [16].

\begin{tabular}{|c|c|c|}
\hline time & $\alpha_{3}\left[\mathrm{eV}^{2}\right]$ & $\tau_{3} / m_{3}[\mathrm{~s} / \mathrm{eV}]$ \\
\hline 3 years & $<4.6 \times 10^{-6}$ & $>1.4 \times 10^{-10}$ \\
\hline 10 years & $<2.6 \times 10^{-6}$ & $>2.5 \times 10^{-10}$ \\
\hline
\end{tabular}

Table 8.3: Expected 90\% C.L. limits from ORCA after 3 and 10 years of running time.

in the $\nu_{\mu} \rightarrow \nu_{e}$ channel, achieved switching the fitted atmospheric mixing angle from the upper to the lower octant.

From Fig. 8.3, one can read off the expected limits on the invisible neutrino decay from ORCA, summarized in Tab. 8.3 for 3 and 10 years of running time, too. Using the relation $\alpha_{3}=m_{3} / \tau_{3}$, it is straightforward to convert the bounds on $\alpha_{3}$ to limits on $\tau_{3} / m_{3}$, in order to compare with the existing limits in the literature, discussed in Sec. 8.1. We find that, within the three-neutrino framework, ORCA could improve the current bounds on $\alpha_{3}$ from oscillation experiments by approximately two orders of magnitude. 


\subsubsection{Effect of decay on standard oscillation parameters}

The existence of invisible neutrino decay could affect the determination of the standard oscillation parameters in ORCA. Here we perform an analysis with the $\chi^{2}$ function in Eq. 8.11 marginalized with respect to the decay constant $\alpha_{3}$,

$$
\chi_{\text {atm }}^{2}\left(\sin ^{2} \theta_{23}, \Delta m_{31}^{2}\right)=\min _{\alpha_{3}}\left[\chi^{2}\left(\sin ^{2} \theta_{23}, \Delta m_{31}^{2}, \alpha_{3}\right)\right],
$$

for each pair of values $\left(\sin ^{2} \theta_{23}, \Delta m_{31}^{2}\right)$, where $\alpha_{3}$ is varied freely in the fit. The result of our simulation is presented in Fig. 8.4, where one can see that neutrino decay does not affect the sensitivity of ORCA to the mass splitting, and only worsens very slightly the sensitivity to the atmospheric angle.

We have also tested the implications of neutrino decay in the ability of ORCA to determine the neutrino mass ordering. To do so, we have simulated data for a given true ordering (TO) of neutrino masses and different assumed true values of the decay constant, $\alpha_{3}^{\text {true }}: N_{i j}^{\text {dat }}\left(\sin ^{2} \theta_{23}^{\mathrm{BF}}, \Delta m_{31}^{2 \mathrm{BF}}, \alpha_{3}^{\text {true }}, \mathrm{TO}\right)$, where we have fixed the oscillation parameters to their best fit value in Tab. 8.1. Then, we evaluate the $\chi^{2}$ function in Eq. 8.11) assuming the other (wrong) mass ordering (WO) and marginalize over the two oscillation parameters as well as the decay constant $\alpha_{3}$, with $N_{i j}\left(\sin ^{2} \theta_{23}, \Delta m_{31}^{2}, \alpha_{3}\right.$, WO $)$.

Our results, presented in Fig. 8.5, show ORCA's sensitivity to the ordering of the neutrino mass spectrum when the true ordering is assumed to be normal (blue lines) and inverted (magenta lines). In the limit of very small $\alpha_{3}$, we recover the standard ORCA sensitivity to the mass ordering, as expected. From the figure, we can also appreciate a reduction of ORCA's mass ordering discrimination power for $\alpha_{3} \sim 10^{-4} \mathrm{eV}^{2}$, when true normal mass ordering is assumed. For values of $\alpha_{3}$ larger than $10^{-4} \mathrm{eV}^{2}$, a general increase on the neutrino mass ordering sensitivity is obtained. Note, however, that these values of the decay constant are already excluded by current oscillation limits. On the other hand, this enhanced sensitivity to the mass ordering arises from the different oscillatory patterns between NO and IO induced by the neutrino decay, rather than by matter effects and neutrino flux differences. In any case, in the region of interest where the experiment is most sensitive, corresponding to $\alpha_{3} \sim 10^{-6} \mathrm{eV}^{2}$, the sensitivity to the neutrino mass ordering is robust and does not show a large dependence on the decay. Notice that the robustness of ORCA's sensitivity to the neutrino mass ordering is mostly due to the broad number of baselines and energies accessible to atmospheric neutrinos. In consequence, this result can not be automatically extended to the case of accelerator-based long-baseline oscillation experiments, where the fixed baselines and narrow energy spectrum might hinder the ability of 



Figure 8.4: ORCA sensitivity to the atmospheric oscillation parameters in the standard picture (filled regions) and with neutrino decay after marginalizing over $\alpha_{3}$ (black lines) at $95 \%$ and $99 \%$ C.L. after 3 years (left panel) and 10 years (right panel) of running time. Figure taken from Ref. [16].

disentangling the effect of flavor oscillations from that of neutrino decay. Dedicated analysis will be required to investigate the impact of neutrino decay on the mass ordering sensitivity of these experiments.

\subsubsection{Other future prospects for invisible neutrino decay}

The sensitivity to the invisible neutrino decay at different future experiments has been recently estimated at several studies. Here we will summarize the most relevant results we have found in the literature.

Ref. [323] analyzes the sensitivity of the future long-baseline neutrino experiment DUNE. Considering a run of 5 years in neutrino mode plus other 5 years in antineutrino mode, it is shown that a bound of $\tau_{3} / m_{3}>4.50 \times 10^{-11} \mathrm{~s} / \mathrm{eV}$ could be obtained at $90 \%$ C.L. for the case of normal mass ordering. See also Ref. [324] for a similar analysis.

The authors of Ref. [325], on the other hand, study the sensitivity to neutrino decay focusing on reactor experiments. They find that a bound of $\tau_{3} / m_{3}>$ $9.1 \times 10^{-11} \mathrm{~s} / \mathrm{eV}$ at $90 \%$ C.L. could be obtained after 5 years of run time in JUNO. Note, however, that in this work the authors performed their analysis marginalizing over $1 \sigma$ ranges of neutrino oscillation parameters and, therefore, a marginalization over the $3 \sigma$ ranges could worsen this bound. 


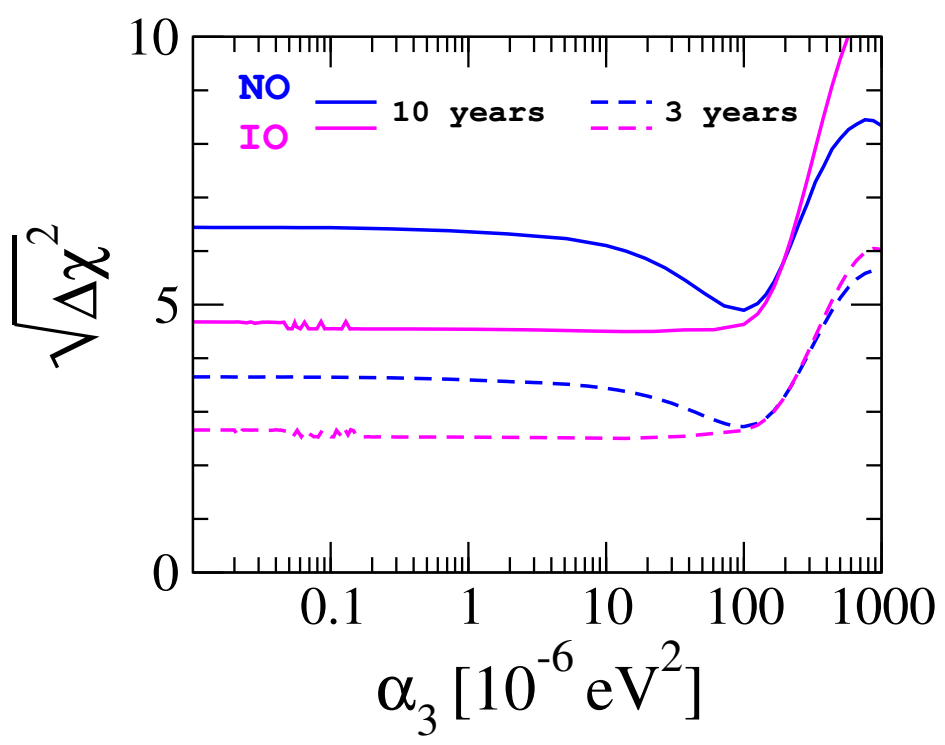

Figure 8.5: ORCA sensitivity to the neutrino mass ordering as a function of the decay constant $\alpha_{3}$. Blue (magenta) lines correspond to the case in which the true ordering is assumed to be normal (inverted), and solid (dashed) lines correspond to an exposure time of 10 years (3 years). Figure taken from Ref. [16]. 
Concerning atmospheric neutrinos, the sensitivity to the invisible neutrino decay at the proposed India-based Neutrino Observatory (INO) has been analyzed in Ref. [326]. After 10 years of data taking, it is found that INO could put a limit of $\tau_{3} / m_{3}>1.51 \times 10^{-10} \mathrm{~s} / \mathrm{eV}$ at $90 \%$ C.L.

Therefore, assuming that ORCA will run for ten years, we find that, besides improving the current bounds on the invisible neutrino decay by approximately two orders of magnitude, it will be, at least, as competitive as DUNE, JUNO and INO, if not a bit better. 


\section{Conclusions}

Neutrino masses and mixing provide the first departure from the standard model of particle physics. With tiny, but yet non-zero, masses, neutrino flavor oscillations occur, as observed with undoubted evidence. In Chapter 1, we have seen how to describe these oscillations in vacuum and in matter. We discussed several approximations to calculate the neutrino oscillation probability in constant matter, finding that DMP [39, 40 gives the most accurate results. As of now, most of the oscillation parameters in the standard 3-neutrino framework are very well measured. We have discussed in detail the status of the remaining unknowns, namely neutrino mass ordering, CP violation and octant discrimination, analyzing the interplay between the different neutrino oscillation data samples. Thanks to the combination of T2K neutrino and antineutrino data, we have now an improved sensitivity to $\mathrm{CP}$ violation. Indeed, $\mathrm{T} 2 \mathrm{~K}$ is the first experiment showing a sensitivity on its own, excluding some values of $\delta$ before combining with reactor data. In the analysis of Chapter 2, we have obtained a strong preference for values of the $\mathrm{CP}$ phase in the range $[\pi, 2 \pi]$, excluding values close to $\pi / 2$ at more than $4 \sigma$. Concerning the octant of $\theta_{23}$, the global analysis prefers the second octant slightly. We have found that, for normal neutrino mass ordering, the upper atmospheric octant is now preferred with $\Delta \chi^{2}=1.6$, while for the case of inverted ordering, values of the atmospheric mixing angle in the lower octant are allowed with $\Delta \chi^{2} \geq 3.2$. More remarkably, our global analysis favors the normal mass ordering over the inverted one at 3.4 $\sigma$. Part of the sensitivity to the mass ordering comes from the recent atmospheric analysis of Super-K. This new analysis shows a preference for normal over inverted mass ordering with $\Delta \chi^{2}=3.5$. On the other hand, a mismatch between the values of $\theta_{13}$ preferred by long-baseline and reactor data (larger for IO) also gives a relevant contribution to the global sensitivity to the mass ordering. This effect is also enhanced due to a tension between the preferred values of the atmospheric mass splitting by $\mathrm{T} 2 \mathrm{~K}$ and reactor experiments. 
In Chapters 4 and 5, we have discussed how this standard neutrino oscillation picture will evolve in the next 15 years approximately. By then, all of the oscillation parameters (except $\delta$ ) will be measured with below $1 \%$ precision. The measurement of CP violation will improve considerably in DUNE and T2HK and also the neutrino mass ordering will be measured in future atmospheric, long baseline and reactor neutrino oscillation experiments.

We also explored the advantages of muon capture on Argon nuclei, a process that improves the sensitivity to the neutrino mass ordering using atmospheric neutrino events at the Liquid Argon Time Projection Chamber DUNE far detector. This is a very relevant result, since it comes without any extra cost. Furthermore, it can be combined with DUNE beam neutrino results, allowing for an enhancement in the total sensitivity to the mass ordering determination. It is important to notice that this result is applicable to any experiment using Argon. In the case of accelerator-based neutrinos, where significant $\nu_{\mu}$ contamination exists in the $\bar{\nu}_{\mu}$ beam, statistical neutrino/antineutrino separation based on muon capture could also be used to enhance DUNE oscillation sensitivities.

The results of these chapters have, however, all been obtained in the case of standard oscillations. Being the CPT theorem one of the few solid predictions of local relativistic quantum field theories, the implications of its potential violation cannot be underestimated. We have shown in Chapter 3 that the impressive limit on CPT violation from the neutral kaon system, normally referred to as the world best bound on CPT invariance violation, turns out to be very weak if viewed as a constraint on the mass squared. For a true test one has to turn to neutrino oscillation experiments, where stronger bounds can be obtained. As discussed then in Chapter 6. DUNE has the capability of obtaining the best limit on the possible CPT violation in mass-squared of particles and antiparticles testing the region where a potential CPT violation arising from non-local quantum gravity, which is suppressed by Planck scale, might be within reach.

As we have explicitly shown, the separate analysis of the neutrino and antineutrino runs is not an option. Otherwise, imposter solutions which do not capture the physics of either mode crop up in the joint analysis. The impact of a potential CPT violation is such that it is imperative to distinguish it from any other unknown physics that can lead to similar experimental signatures. NSI are a simple way to parameterize many types of new physics which may be relevant to neutrino oscillations. We have shown that, although NSI can mimic fake CPT violation, its experimental signature can be distinguished from a genuine CPT violation due to the bounds on the strength of the NSI arising from other experiments. Indeed, we have found that the different results for the neutrino and 
antineutrino parameters measured by the T2K Collaboration may be interpreted in terms of a CPT-conserving scenario in combination with neutrino NSI with matter. However, this equivalence has a caveat, since the size of the diagonal NSI coupling required, $\epsilon_{\tau \tau}^{m} \simeq-0.3$, is excluded by current neutrino oscillation data. Therefore, the future cannot be more exciting. If the slight indications of CPT violation in the mixing angles offered by the separate analysis of the neutrino and antineutrino data sets by T2K are confirmed by the DUNE experiment, genuine CPT violation, i.e. the one that challenges our understanding of Nature in terms of local relativistic quantum field theory, will be the only answer.

In Chapter 7, we have studied the sensitivity of the DUNE and JUNO experiments to quasi-Dirac neutrino oscillations. We have found that, within this scenario, the determination of the neutrino mixing angles becomes much more complicated, if not impossible, even for next-generation experiments such as DUNE or JUNO. The main reason is that the new angles and phases are strongly correlated to the corresponding ones in the active sector, leading to very strong degeneracies. Despite these degeneracies affecting the angles, we have seen that we can define new observables which clearly allow to distinguish the standard oscillation case from the quasi-Dirac neutrino scenario. While most of the parameter space for these observables is still allowed at present, we have shown that DUNE and JUNO can considerably improve the current bounds on these quantities. We have also seen that, if quasi-Dirac neutrino oscillations are real, the new generation of experiments will have the potential to discover quasi-Dirac neutrinos, which would be a big breakthrough in particle physics.

Finally, in Chapter 8, we have performed an analysis of the ORCA experiment in the context of invisible neutrino decay. We have found that, the data on atmospheric neutrinos to be obtained in ORCA in the $\mathrm{GeV}$ energy range could improve the bounds on the decay constant from current oscillation experiments, when including three-neutrino oscillations, by roughly a factor of 100. After ten years, ORCA could constrain the decay parameter to $\tau_{3} / m_{3}>2.5 \times 10^{-10} \mathrm{~s} / \mathrm{eV}$ at $90 \%$ C.L. This means that, in the context of future neutrino oscillation experiments, ORCA will better constrain the invisible neutrino decay in comparison to other experiments such as DUNE, JUNO or INO, although not very significantly. We have also shown that the decay of the heaviest neutrino would not affect the determination of the atmospheric oscillation parameters at ORCA. In particular, the determination of the neutrino mass ordering would remain very robust against the presence of invisible neutrino decay.

Summing up, we have discussed the current status of neutrino oscillations in the standard picture and, using a similar data set, bounded CPT violation in the 
neutrino sector. We have also shown how the determination of the oscillation parameters will improve in the future and finally discussed the sensitivity of DUNE, JUNO and ORCA to different new physics scenarios. 


\section{Appendix A}

\section{Neutrino oscillation parameters 2017}

In Chapter 2 we discussed the results published in Ref. 2]. Here we present the results from a former global fit, prepared in August 2017. We will not enter into too much details, but rather discuss the differences in data considered. In the following section, we discuss the data sets which do not coincide with the ones from Chapter 2. If some experiment is not mentioned, it is automatically assumed that the data sample analyzed coincides with the one discussed before.

\section{A.1 Data included in the 2017 fit}

\section{Reactors}

The 2017 version of our global fit included 500 days of RENO data 327] instead of the 1500 days included in Chapter 2. The analysis was performed the same way as before. In Fig. A.1 we see the larger RENO regions from this analysis, compared to the ones from Daya Bay and Double Chooz, which are the same.

\section{Atmospheric experiments}

In August 2017, the $\chi^{2}$ grid for Super-K phases I-IV was not available yet. For this reason we included only Super-K phases I-III [328]. The main difference is that, in this analysis, Super-K had no sensitivity to $\delta, \theta_{13}$ and neither to the neutrino mass ordering. Therefore, the Super-K data was only affecting the determination of atmospheric neutrino oscillation parameters. In Fig. A.2 we 

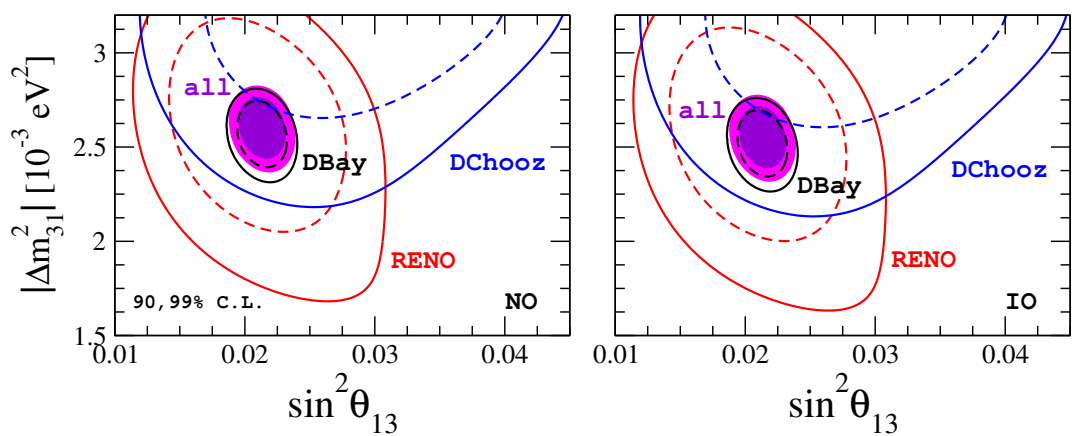

Figure A.1: 90 and 99\% C.L. (2 d.o.f.) allowed regions at the $\sin ^{2} \theta_{13^{-}}-\Delta m_{31}^{2}$ with old RENO data. The left (right) panels correspond to normal (inverted) mass ordering.

show the results from this analysis comparing with the results from DeepCore and ANTARES discussed in Chapter 2.

\section{Long-baseline accelerators}

Our 2017 analysis included the first T2K anti-neutrino sample, as well as data in the neutrino mode [103,104. We used the combined analysis with a total of $7.48 \times 10^{20} \mathrm{POT}$ in the neutrino run and $7.47 \times 10^{20}$ POT in the antineutrino run

Regarding $\mathrm{NO} \nu \mathrm{A}$, we used data corresponding to $6.05 \times 10^{20}$ POT 110,111 in neutrino mode. The main difference with respect to the later fit was that maximal atmospheric mixing was disfavored at around $2.6 \sigma$. This feature can be seen in Fig. A.3 by the two separated regions in $\mathrm{NO} \nu \mathrm{A}$. 

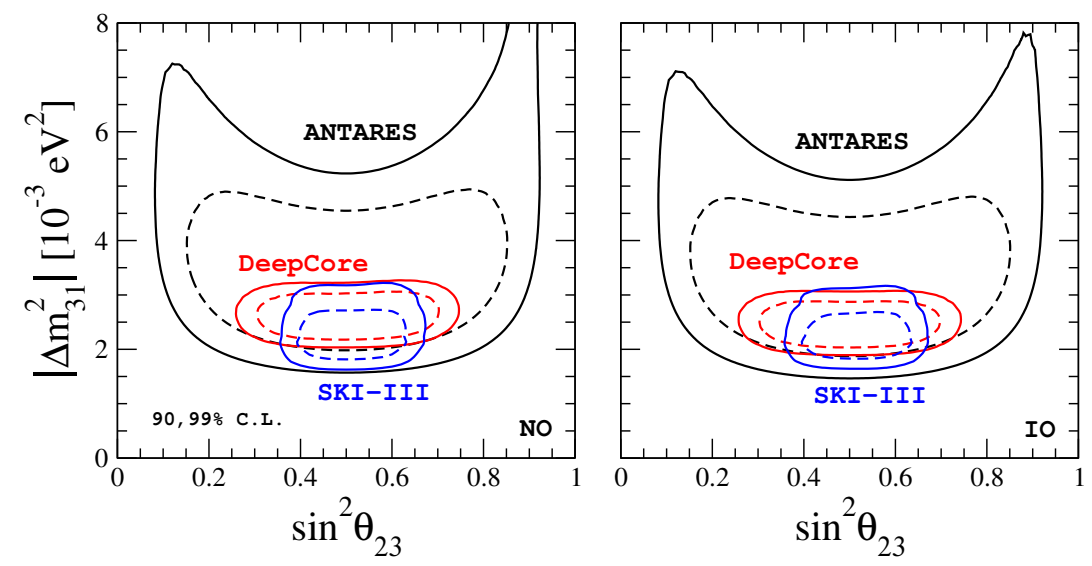

Figure A.2: 90 and 99\% C.L. (2 d.o.f.) allowed regions at the $\sin ^{2} \theta_{23}-\Delta m_{31}^{2}$ plane obtained from the atmospheric neutrino experiments for normal (left) and inverted ordering (right) with only SK I-III.



Figure A.3: 90 and 99\% C.L. (2 d.o.f.) allowed regions at the $\sin ^{2} \theta_{23}-\Delta m_{31}^{2}$ plane for normal (left) and inverted mass ordering (right) as restricted from the long-baseline experiments.

\section{A.2 Global fit 2017}

The results obtained in this former global neutrino oscillation fit are summarized in Table A.1 as well as in Figs. A.4 and A.5 for normal and inverted mass ordering. Thanks to the combination of $\mathrm{T} 2 \mathrm{~K}$ neutrino and antineutrino 
data, we had for the first time sensitivity to $\mathrm{CP}$ violation coming from one single experiment on its own.

\begin{tabular}{|lcc|}
\hline parameter & best fit $\pm 1 \sigma$ & $3 \sigma$ range \\
\hline$\Delta m_{21}^{2}\left[10^{-5} \mathrm{eV}^{2}\right]$ & $7.56 \pm 0.19$ & $7.05-8.14$ \\
$\left|\Delta m_{31}^{2}\right|\left[10^{-3} \mathrm{eV}^{2}\right](\mathrm{NO})$ & $2.55 \pm 0.04$ & $2.43-2.67$ \\
$\left|\Delta m_{31}^{2}\right|\left[10^{-3} \mathrm{eV}^{2}\right](\mathrm{IO})$ & $2.47_{-0.05}^{+0.04}$ & $2.34-2.59$ \\
$\sin ^{2} \theta_{12} / 10^{-1}$ & $3.21_{-0.16}^{+0.18}$ & $2.73-3.79$ \\
$\theta_{12} /^{\circ}$ & $34.5_{-1.0}^{+1.1}$ & $31.5-38.0$ \\
$\sin ^{2} \theta_{23} / 10^{-1}(\mathrm{NO})$ & $4.30_{-0.18}^{+0.20}$ & $3.84-6.35$ \\
$\theta_{23} /^{\circ}$ & $41.0^{\circ} 1.1$ & $38.3-52.8$ \\
$\sin ^{2} \theta_{23} / 10^{-1}(\mathrm{IO})$ & $5.98_{-0.15}^{+0.17}$ & $3.89-4.88 \& 5.22-6.41$ \\
$\theta_{23} /^{\circ}$ & $50.7_{-0.9}^{+1.0}$ & $38.6-44.3 \& 46.2-53.2$ \\
$\sin ^{2} \theta_{13} / 10^{-2}(\mathrm{NO})$ & $2.155_{-0.075}^{+0.090}$ & $1.89-2.39$ \\
$\theta_{13} /{ }^{\circ}$ & $8.44_{-0.15}^{+0.18}$ & $7.9-8.9$ \\
$\sin ^{2} \theta_{13} / 10^{-2}(\mathrm{IO})$ & $2.155_{-0.092}^{+0.076}$ & $1.90-2.39$ \\
$\theta_{13} /{ }^{\circ}$ & $8.44_{-0.18}^{+0.15}$ & $7.9-8.9$ \\
$\delta / \pi(\mathrm{NO})$ & $1.40_{-0.20}^{+0.31}$ & $0.00-2.00$ \\
$\delta /{ }^{\circ}$ & $252_{-36}^{+56}$ & $0-360$ \\
$\delta / \pi(\mathrm{IO})$ & $1.56_{-0.26}^{+0.22}$ & $0.00-0.17 \& 0.83-2.00$ \\
$\delta /{ }^{\circ}$ & $281_{-47}^{+39}$ & $0-31 \& 149-360$ \\
\hline
\end{tabular}

Table A.1: Neutrino oscillation parameters summary determined from the 2017 global analysis. The ranges for inverted ordering refer to the local minimum of this neutrino mass ordering.

Concerning the octant of $\theta_{23}$, this global analysis preferred the lower octant, in contrast to the one in Chapter 2. Note that, in both cases, this preference is not very significant. We found that, for normal neutrino mass ordering, the lower atmospheric octant was preferred with $\Delta \chi^{2}=2.1$, while for the case of inverted ordering we obtained a local minimum in the second octant at $\Delta \chi^{2}=4.3$ with respect to the global minimum. Maximal atmospheric mixing was disfavored at $\Delta \chi^{2}=6.0$ for the case of normal ordering. The global fit showed a slight preference for normal neutrino mass ordering, with $\Delta \chi^{2}=4.3$. This sensitivity to the mass ordering came partly from a tension in the preferred values of $\theta_{23}$ in $\mathrm{T} 2 \mathrm{~K}$ and $\mathrm{NO} \nu \mathrm{A}$, found to be stronger for the case of inverted mass ordering. 
Note also that the mismatch between the values of $\theta_{13}$ preferred by long-baseline and reactor data for IO also gives a relevant contribution to the global sensitivity to the mass ordering as is the case in Chapter 2 .
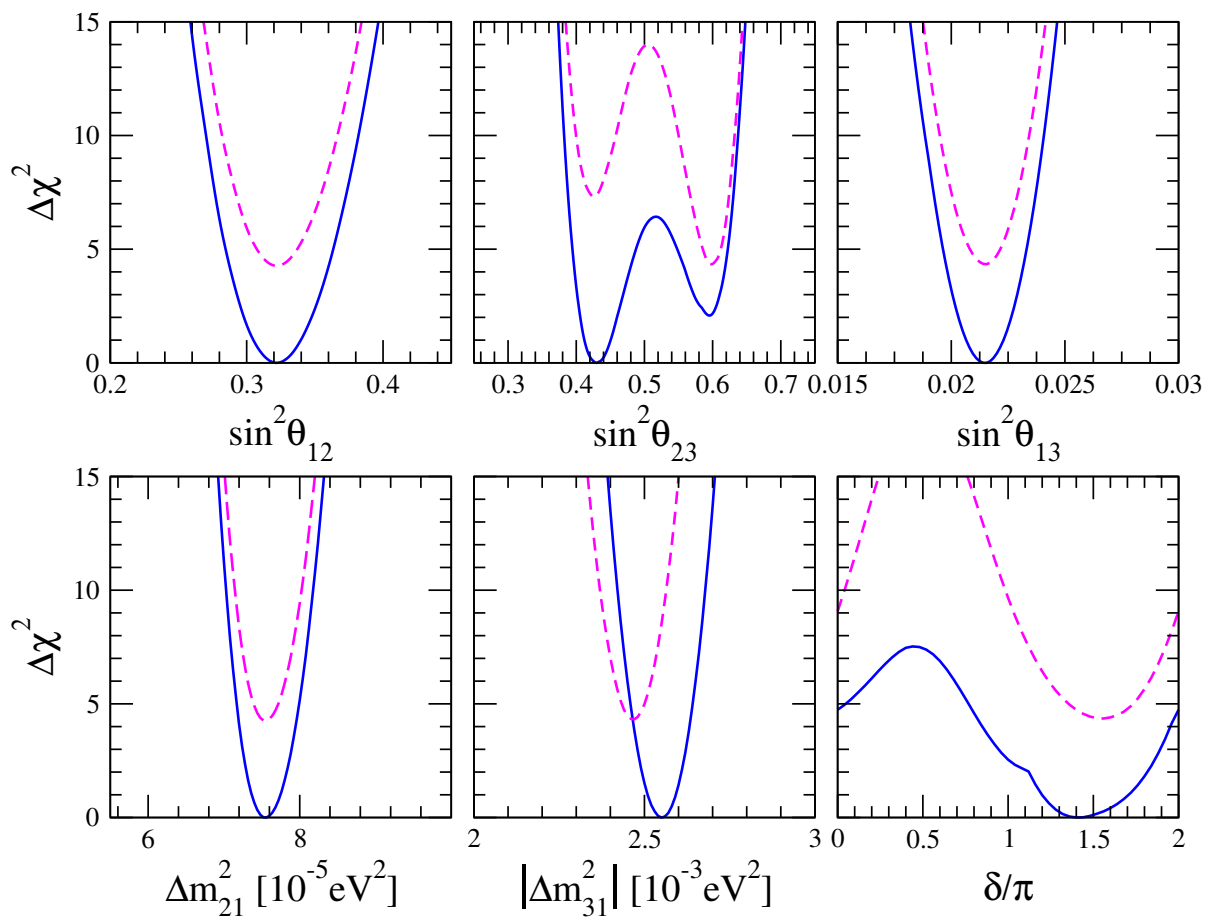

Figure A.4: Summary of neutrino oscillation parameters, 2017. Blue (solid) lines correspond to NO and magenta (dashed) lines to IO. 

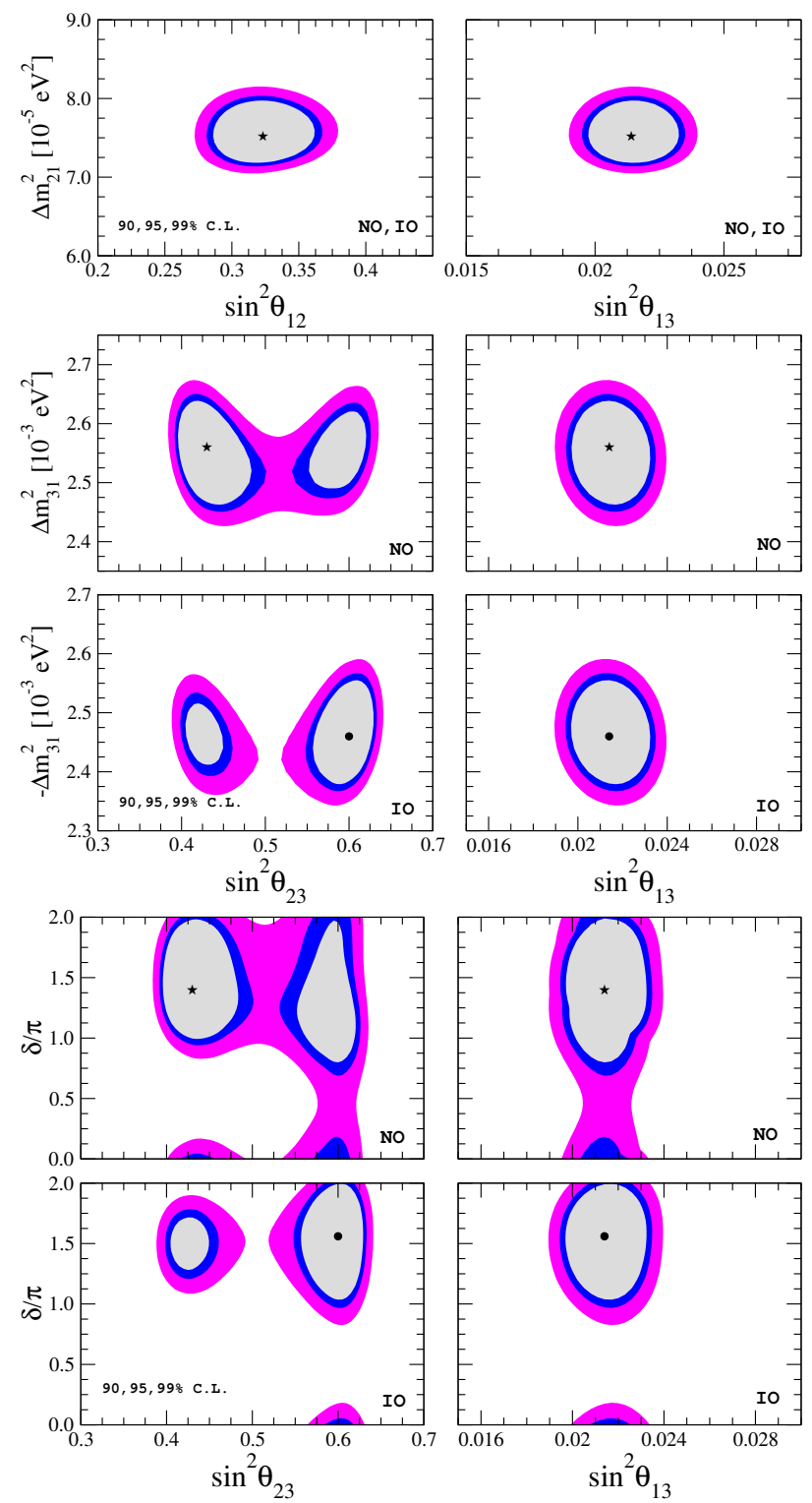

Figure A.5: Global Fit summary 2017. In the two four-panel figures, the upper ones correspond to normal ordering and the lower ones to inverted mass ordering. Global fit regions correspond to 90, 95 and 99\% C.L. (2 d.o.f.). 


\section{Appendix B}

\section{Neutrino oscillation parameters 2020}

Currently, we are working on an update of Ref. 2]. Here we present the preliminary results of this new global fit. Since no combined analysis has been performed yet, here we only discuss the most recent data and show the updated results of the analyses of single experiments.

\section{B.1 Data to be included in the 2020 fit}

\section{Reactors}

For the new global fit, we update our analysis of RENO and Daya Bay. In the case of RENO, we include now 2200 days of live data [83, 50\% more data than in Chapter 2. Also the analysis of Daya Bay has been updated. We now use 1958 days of data 84. Both experiments have further improved their sensitivity to measure $\Delta m_{31}^{2}$ and $\sin ^{2} \theta_{13}$. The results of our updated analysis are shown in Fig. B.1. As can be seen, the analysis will still be dominated by Daya Bay.

\section{Atmospheric experiments}

For the next global fit, we update the analysis of DeepCore data. The experiment includes now, in addition to track-like events, shower-like events, which increases the number of events from roughly 6000 [93] to around 20000 [135, 329]. The analyzed data correspond to 3 years of observation time from April 2012 to May 2015. The analysis is described in Ref. [329] and the new data sample can be downloaded from Ref. [330]. The result of our analysis is shown in Fig. B.2. 

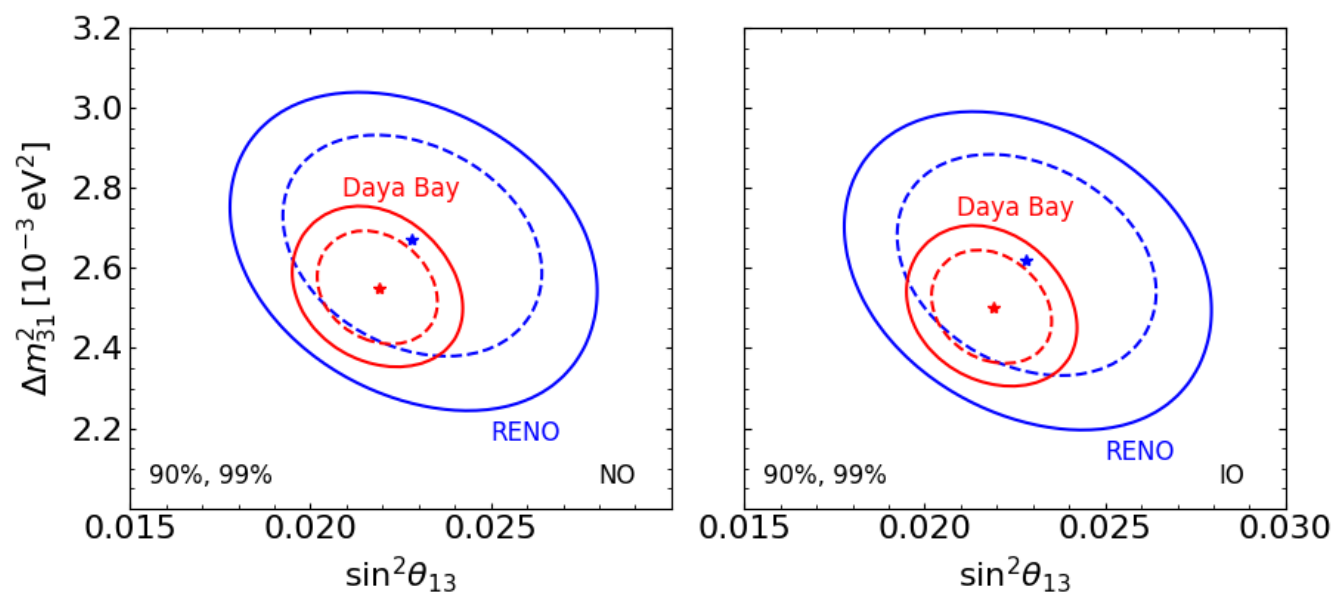

Figure B.1: 90 and 99\% C.L. (2 d.o.f.) allowed regions at the $\sin ^{2} \theta_{13^{-}}-\Delta m_{31}^{2}$ for a new 2020 global fit. The best fit points are indicated with stars. The left (right) panels correspond to normal (inverted) mass ordering.

\section{Long-baseline accelerators}

We have also updated our analysis of $\mathrm{T} 2 \mathrm{~K}$ and $\mathrm{NO} \nu \mathrm{A}$. In the case of $\mathrm{T} 2 \mathrm{~K}$, the updated data sample corresponds to an exposure at Super-Kamiokande of $1.49 \times 10^{21}$ POT in neutrino mode and $1.63 \times 10^{21}$ POT in antineutrino mode and has been collected from January 2010 until June 2018 331, 332. T2K observed 243 (140) muon (anti-muon) events and 75 (15) electron (positron) events. In addition, there are 15 electron events where also a pion is produced. These results improve the former results [164, allowing now to exclude CP-conserving values of $\delta$ at close to $3 \sigma$ confidence level.

On the other hand, $\mathrm{NO} \nu \mathrm{A}$ has also released data in antineutrino mode. They use now $8.85 \times 10^{20}$ POT in neutrino mode 102 and $12.33 \times 10^{20}$ POT in antineutrino mode 333. NO $\nu$ A finds 113 (102) muon (anti-muon) events in the disappearance channel, expecting 730 (476) without oscillations, and 58 (27) electron (positron) events in appearance channel. The 27 events in antineutrino mode consist the first ever significant observation of $\bar{\nu}_{e}$ appearance in a long baseline experiment. Unlike in $\mathrm{T} 2 \mathrm{~K}$, in $\mathrm{NO} \nu \mathrm{A}$ the best fit is obtained for $\delta=0$, which is in tension with the $\mathrm{T} 2 \mathrm{~K}$ result. However, the $\mathrm{NO} \nu \mathrm{A}$ data can not exclude any value of $\delta$ with meaningful significance at the moment and, therefore, this new best fit value might be due to a statistical fluctuation. In both cases, the measurement worsens when relaxing the reactor prior on $\theta_{13}$, which is used in 

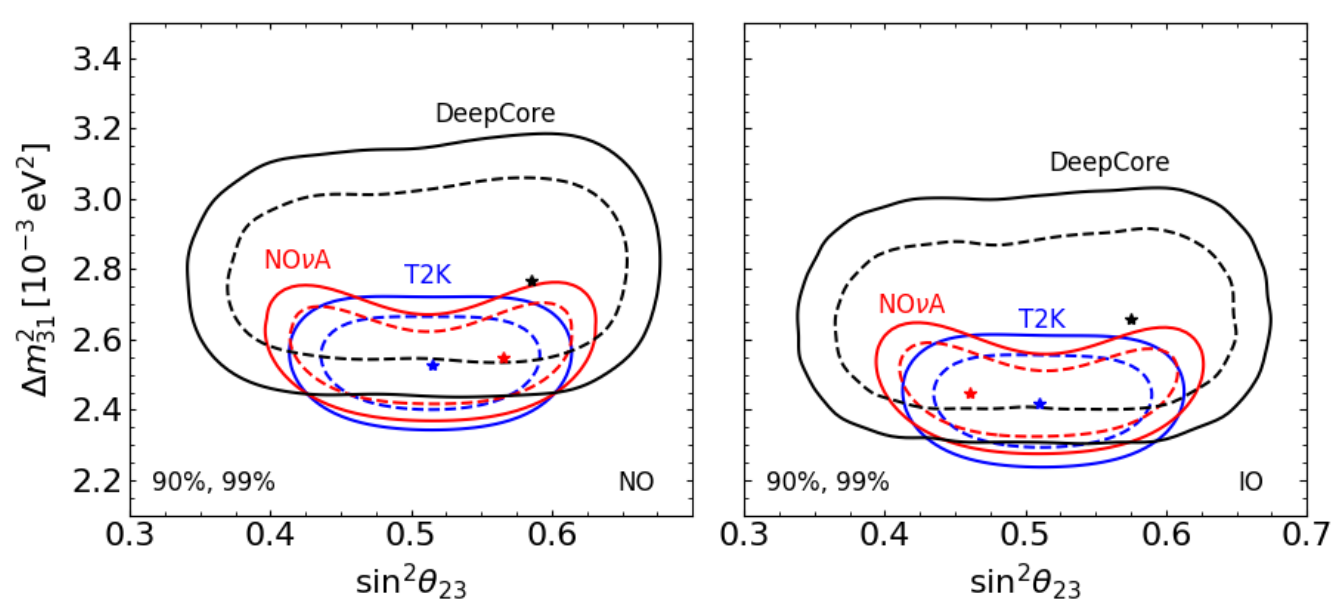

Figure B.2: 90 and $99 \%$ C.L. (2 d.o.f.) allowed regions at the $\sin ^{2} \theta_{23}-\Delta m_{31}^{2}$ plane for normal (left) obtained from the updated analyses of $\mathrm{T} 2 \mathrm{~K}, \mathrm{NO} \nu \mathrm{A}$ and DeepCore. The best fit points are indicated with stars.

both experiments. The results of our new analysis (without prior) are plotted in Figs. B.2 and B.3. As can be seen, the accelerator experiments give more precise results in the determination of the atmospheric neutrino oscillation parameters than DeepCore. However, note that the analysis of DeepCore still does not include the full 10 year data set, which has not been released yet. Maybe with the full data set DeepCore will become competitive with the long-baseline accelerators. In the case of $\mathrm{T} 2 \mathrm{~K}$ and $\mathrm{NO} \nu \mathrm{A}$, we can also measure $\theta_{13}$ and have some sensitivity to $\delta$. Note that, in the upcoming global fit these results will be combined with reactor data, improving also the determination of $\delta$, by bounding the allowed region for $\theta_{13}$. In terms of neutrino mass ordering, both experiments prefer normal ordering with $\Delta \chi^{2} \approx 1.6$. As in the case of $\delta$, this will improve further after combining with reactors. Likewise, the addition of the atmospheric data set will further increase the sensitivity to the neutrino mass ordering. 

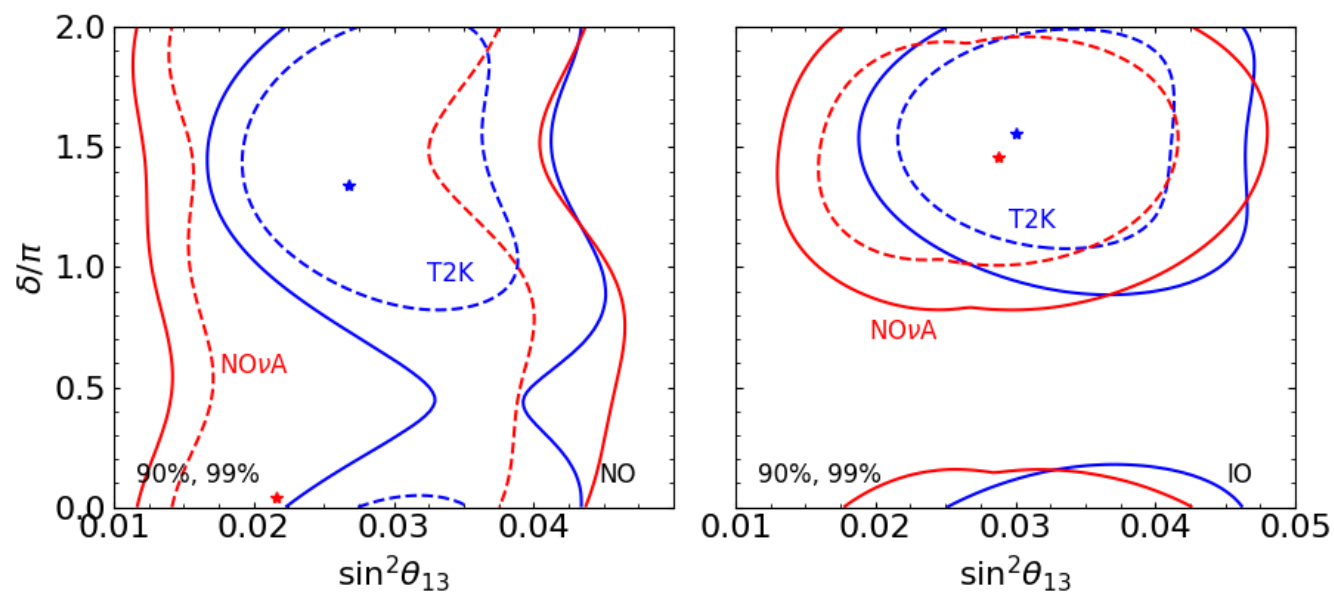

Figure B.3: 90 and 99\% C.L. (2 d.o.f.) allowed regions at the $\sin ^{2} \theta_{13^{-}} \delta$ plane obtained from the analysis of $\mathrm{T} 2 \mathrm{~K}$ and $\mathrm{NO} \nu \mathrm{A}$ data. 


\section{Statement of contributions}

Since large parts of the thesis are based on published papers, here, I summarize my contribution to each chapter of the thesis. First, regarding the writing, I participated in the writing of the text for all the papers. My contribution to the text grew over time. While in the first papers I was writing smaller sections (for example, CPT violation at DUNE), I wrote large part of some of the text in later papers (for example, quasi-Dirac neutrinos), as I was gaining more experience.

Regarding the analyses:

1. The analysis for this chapter was performed by several persons. Some of the expressions are very large and it is easy to make mistakes when copying them. Therefore we made first sure to obtain the same results among ourselves. This means that, in cooperation with the other co-authors, I produced all of the main results of this paper.

2. I performed the analysis of data coming from the atmospheric experiments DeepCore and ANTARES, and from the accelerator experiments T2K and $\mathrm{NO} \nu \mathrm{A}$. For the analysis of atmospheric data I developed my own Fortranroutines and cross-checked my results with the ones obtained by my colleague Pablo Fernández de Salas. For accelerators I used GLoBES changing the computation of the $\chi^{2}$ to allow for the analysis of real data, and I was the only person in charge of this part of the analysis. I also worked here closely with my supervisor in deciding which combinations of data to show and I created the figures for the paper.

3. Similarly to the previous point, I was in charge of the $\mathrm{T} 2 \mathrm{~K}$ and $\mathrm{NO} \nu \mathrm{A}$ data analyisis (since the data samples are those analysed in Appendix A), but separating neutrinos from antineutrinos. In addition I calculated the $\Delta x$ profiles from the profiles obtained from the neutrino and antineutrino profiles. The results of this chapter are more detailed here than in the actual paper, where the focus was set on DUNE. 
4. There is no analysis in this chapter.

5. The first part of this chapter was originally performed only for this thesis. For the second part of the chapter (based on Ref. [10]), the analysis was divided in two parts. First, we analyzed the propagation of events from the atmosphere to the detector and then we performed a statistical analysis. My contribution to the first part, where the number of events as a function of oscillation parameters was calculated, was small. However, I contributed significantly to the development of the numerical code for the statistical analysis, where I performed the minimization over systematic uncertainties in the analysis using python MINUIT.

6. I learned to use GLoBES when making the analysis of the first part of this chapter. Therefore, I developed my "GLoBES core-code" which I was continuously improving since then. Note that even though this paper came out after the global fit, the analyses were performed more or less in parallel. So I used similar core-codes for DUNE in this paper and $\mathrm{NO} \nu \mathrm{A}$ and $\mathrm{T} 2 \mathrm{~K}$ in the global fit. For the second part of the chapter, I used for the first time snu.c to include NSI in the analysis. I had to get familiar with snu.c and therefore this work was very useful for understanding how to change the neutrino oscillation probability in GLoBES.

7. For the analysis of this chapter I had to change for the first time snu.c. This gave me a lot of insight on how snu.c actually works. I used then my DUNE codes in combination with the modified version of snu.c to perform the DUNE analysis. The JUNO $\chi^{2}$ could be parameterized as a function of the mixing angles for this paper. While this was done by someone else, I used the results to make the combination with the DUNE analysis.

8. I wrote the piece of code which calculated the neutrino oscillation probability with neutrino decay. Even though the main analysis was performed by someone else, I contributed with advice and discussion in the development of this code. The final plots were created by me. 


\section{Resumen de la Tesis}

El tema principal de esta tesis es la fenomenología de neutrinos. Se trata de uno de los campos de investigación más activos, que interconecta la física de partículas, la astrofísica y la cosmología. Aquí nos enfocamos en la fenomenología de las oscilaciones de neutrinos, que resultan de la superposición cuántica de los autoestados de masa y de mezcla. Para poder oscilar, por lo menos dos neutrinos tienen que tener masa. Por lo tanto, la observación de las oscilaciones de neutrinos confirma que son masivos, lo cual constituye la primera evidencia de física más allá del modelo estándar. Sin embargo, el mecanismo que crea la masa de los neutrinos es desconocido, aunque muchos modelos han sido propuestos. En estos modelos, que pueden ser probados en experimentos actuales y futuros, el contenido de partículas tiene que ser extendido respecto al modelo estándar y también nuevas interacciones son posibles. Entonces, la descripción más sencilla de las oscilaciones de neutrinos, el paradigma de tres neutrinos, podría estar incompleto. Desafortunadamente, los experimentos actuales no tienen la sensibilidad suficiente para detectar efectos subdominantes. Quizá será posible verlos en experimentos futuros. Durante este doctorado se ha estudiado varios escenarios de oscilaciones estándar y también de oscilaciones más allá del paradigma de tres neutrinos. La tesis está dividida en dos partes: primero hablamos del estado actual de la determinación de los parámetros que describen las oscilaciones de los neutrinos. En la segunda parte hablamos de las sensibilidades de experimentos futuros para mejorar estas medidas y además vemos las sensibilidades de algunos experimentos para descubrir efectos de nueva física.

\section{Oscilaciones de neutrinos}

En el capítulo 1 hemos derivado las fórmulas que describen las oscilaciones de los neutrinos. Hemos visto que, en el caso de tres neutrinos, las oscilaciones 
dependen de 6 parámetros: $\Delta m_{21}^{2}, \Delta m_{31}^{2}, \theta_{12}, \theta_{13}, \theta_{23}$ y $\delta$. La probabilidad de conversión de un sabor inicial $\alpha$ a un sabor $\beta$ está dada por

$$
\begin{aligned}
P_{\alpha \beta}(E, L) & =\delta_{\alpha \beta}-4 \sum_{k>j} \operatorname{Re}\left[U_{\alpha k}^{*} U_{\beta k} U_{\alpha j} U_{\beta j}^{*}\right] \sin ^{2}\left(\frac{\Delta m_{k j}^{2} L}{4 E}\right) \\
& +2 \sum_{k>j} \operatorname{Im}\left[U_{\alpha k}^{*} U_{\beta k} U_{\alpha j} U_{\beta j}^{*}\right] \sin \left(\frac{\Delta m_{k j}^{2} L}{2 E}\right),
\end{aligned}
$$

donde $E$ es la energía del neutrino y $L$ la distancia recorrida. La matriz de mezcla leptónica está dada por

$$
\begin{aligned}
U & =\left(\begin{array}{ccc}
1 & 0 & 0 \\
0 & c_{23} & s_{23} \\
0 & -s_{23} & c_{23}
\end{array}\right)\left(\begin{array}{ccc}
c_{13} & 0 & s_{13} e^{-i \delta} \\
0 & 1 & 0 \\
-s_{13} e^{i \delta} & 0 & c_{13}
\end{array}\right)\left(\begin{array}{ccc}
c_{12} & s_{12} & 0 \\
-s_{12} & c_{12} & 0 \\
0 & 0 & 1
\end{array}\right) \\
& =\left(\begin{array}{ccc}
c_{12} c_{13} & s_{12} c_{13} & s_{13} e^{-i \delta} \\
-s_{12} c_{23}-c_{12} s_{23} s_{13} e^{i \delta} & c_{12} c_{23}-s_{12} s_{23} s_{13} e^{i \delta} & s_{23} c_{13} \\
s_{12} s_{23}-c_{12} c_{23} s_{13} e^{i \delta} & -c_{12} s_{23}-s_{12} c_{23} s_{13} e^{i \delta} & c_{23} c_{13}
\end{array}\right)
\end{aligned}
$$

donde $s_{i j}=\sin \theta_{i j}$ and $c_{i j}=\cos \theta_{i j}$. Cuando los neutrinos se propagan dentro de materia, estas fórmulas son alteradas por las interacciones de los neutrinos con los electrones dentro del medio. En este caso, es muy difícil derivar fórmulas exactas para las oscilaciones, pero se puede derivar fórmulas aproximadas usando cálculo de perturbaciones en pequeños parámetros cuando la densidad de materia es constante en el trayecto del neutrino. En la parte principal de este capítulo, hemos discutido varias de estas aproximaciones y hemos encontrado que la más exacta es la denominada DMP. Para obtener las expresiones simplemente hay que reemplazar los parámetros de oscilación por sus correspondientes cantidades en 
materia:

$$
\begin{aligned}
\sin ^{2} \widetilde{\theta}_{13} & =\frac{1}{2}\left(1-\frac{\Delta m_{e e}^{2} \cos 2 \theta_{13}-a}{\Delta \widetilde{m}_{e e}^{2}}\right), \\
\Delta \widetilde{m^{2}} e e & =\Delta m_{e e}^{2} \sqrt{\left(\cos 2 \theta_{13}-a / \Delta m_{e e}^{2}\right)^{2}+\sin ^{2} 2 \theta_{13}} \\
\sin ^{2} \widetilde{\theta}_{12} & =\frac{1}{2}\left(1-\frac{\Delta m_{21}^{2} \cos 2 \theta_{12}-a_{12}}{\Delta \widetilde{m}^{2}}\right), \\
\operatorname{con} \quad a_{12} & =\frac{1}{2}\left(a+\Delta m_{e e}^{2}-\Delta \widetilde{m}^{2} e e\right), \\
\Delta \widetilde{m^{2}} 21 & =\Delta m_{21}^{2} \sqrt{\left(\cos 2 \theta_{12}-a_{12} / \Delta m_{21}^{2}\right)^{2}+\cos ^{2}\left(\theta_{13}-\widetilde{\theta}_{13}\right) \sin ^{2} 2 \theta_{12}}, \\
\Delta \widetilde{m^{2}}{ }_{31} & =\Delta m_{31}^{2}+\frac{a}{4}+\frac{1}{2}\left(\Delta \widetilde{m^{2}}{ }_{21}-\Delta m_{21}^{2}\right)+\frac{3}{4}\left(\Delta \widetilde{m^{2}} e-\Delta m_{e e}^{2}\right) .
\end{aligned}
$$

Aquí $a=2 E V$, donde $V=\sqrt{2} G_{F} n_{e}$ es el potencial efectivo en materia que depende de la densidad de electrones en el medio, $n_{e}$.

\section{Los parámetros de oscilación de neutrinos}

En el capítulo 2 discutimos cómo extraer los parámetros de oscilación a partir de los datos experimentales. Para esto se ha de hacer un análisis estadístico de los mismos. La cantidad importante aquí es el número de eventos esperados para un conjunto de parámetros de oscilación, $N^{\text {exp }}$, que se tiene que comparar con el número de eventos observados, $N^{\text {dat }}$. Esta comparación se hace mediante un análisis de $\chi^{2}$. Generalmente, tenemos

$$
\chi^{2}=\sum_{n} \frac{\left(N^{\mathrm{dat}}-N^{\mathrm{exp}}\right)^{2}}{\sigma^{2}}+\chi_{\mathrm{sys}}^{2}
$$

asumiendo estadística Gaussiana, o

$$
\chi^{2}=2 \sum_{n}\left(N^{\exp }-N^{\text {dat }}+N^{\text {dat }} \log \frac{N^{\text {dat }}}{N^{\exp }}\right)+\chi_{\text {sys }}^{2}
$$

asumiendo estadística Poissoniana. Aquí $\chi_{\text {sys }}^{2}$ contiene posibles penalizaciones en el tratamiento de posibles incertidumbres sistemáticas. De forma simplificada, el número de eventos se calcula según 


\begin{tabular}{|lccc|}
\hline parámetro & best fit $\pm 1 \sigma$ & rango de $2 \sigma$ & rango de $3 \sigma$ \\
\hline$\Delta m_{21}^{2}\left[10^{-5} \mathrm{eV}^{2}\right]$ & $7.55_{-0.16}^{+0.20}$ & $7.20-7.94$ & $7.05-8.14$ \\
$\left|\Delta m_{31}^{2}\right|\left[10^{-3} \mathrm{eV}^{2}\right](\mathrm{NO})$ & $2.50 \pm 0.03$ & $2.44-2.57$ & $2.41-2.60$ \\
$\left|\Delta m_{31}^{2}\right|\left[10^{-3} \mathrm{eV}^{2}\right](\mathrm{IO})$ & $2.42_{-0.04}^{+0.03}$ & $2.34-2.47$ & $2.31-2.51$ \\
$\sin ^{2} \theta_{12} / 10^{-1}$ & $3.20_{-0.16}^{+0.20}$ & $2.89-3.59$ & $2.73-3.79$ \\
$\sin ^{2} \theta_{23} / 10^{-1}(\mathrm{NO})$ & $5.47_{-0.30}^{+0.20}$ & $4.67-5.83$ & $4.45-5.99$ \\
$\sin ^{2} \theta_{23} / 10^{-1}(\mathrm{IO})$ & $5.51_{-0.30}^{+0.18}$ & $4.91-5.84$ & $4.53-5.98$ \\
$\sin ^{2} \theta_{13} / 10^{-2}(\mathrm{NO})$ & $2.160_{-0.069}^{+0.083}$ & $2.03-2.34$ & $1.96-2.41$ \\
$\sin ^{2} \theta_{13} / 10^{-2}(\mathrm{IO})$ & $2.220_{-0.076}^{+0.074}$ & $2.07-2.36$ & $1.99-2.44$ \\
$\delta / \pi(\mathrm{NO})$ & $1.32_{-0.15}^{+0.21}$ & $1.01-1.75$ & $0.87-1.94$ \\
$\delta / \pi(\mathrm{IO})$ & $1.56_{-0.15}^{+0.13}$ & $1.27-1.82$ & $1.12-1.94$ \\
\hline
\end{tabular}

Tabla 1: Resumen de los parámetros de oscilación de los neutrinos determinado en este análisis global.

$$
N_{\beta}^{\exp } \sim \sum_{\gamma=e, \mu, \tau} \phi_{\gamma} P_{\gamma \beta} R_{\beta}
$$

Aquí, $\phi_{\gamma}$ es un flujo de neutrinos iniciales de sabor $\gamma, P_{\gamma \beta}$ es la probabilidad de oscilación de un sabor $\gamma$ a otro sabor $\beta$ y $R_{\beta}$ es una función que contiene todos los efectos posibles en los detectores, como secciones eficaces, eficiencias y la reconstrucción de eventos. Para el análisis global, se ha analizado datos de todos los tipos de experimentos de oscilación de neutrinos: experimentos que observan neutrinos solares, neutrinos de reactores nucleares, neutrinos creados en la atmósfera y neutrinos creados en aceleradores de partículas. Los resultados de este análisis están resumidos en la tabla 1 y la figura 1 . Hemos encontrado que los parámetros $\Delta m_{21}^{2}, \theta_{12}$ y $\theta_{13}$ ya están medidos con mucha precisión. Sin embargo, quedan tres parámetros desconocidos: primero, el valor de la fase $\delta$. Gracias a la combinación de datos de neutrinos y de antineutrinos tomados en el experimento T2K, obtenemos por primera vez intervalos cerrados en este parámetro. De hecho, T2K es el primer experimento que puede medir la fase solo, es decir, sin combinar con los resultados de otros experimentos, especialmente de los reactores. Hemos visto que ahora se prefiere valores en el intervalo $[\pi, 2 \pi]$, mientras valores cerca de $0.5 \pi$ quedan desfavorecidos a $\sim 4 \sigma$. Otro desconocido es el octante del ángulo $\theta_{23}$ $\left(\sin ^{2} \theta_{23}<0.5 \mathrm{o} \sin ^{2} \theta_{23}<0.5\right)$. El análisis global prefiere ligeramente el segundo octante, aunque soluciones en el primer octante son posibles con $\Delta \chi^{2}<1.6$ para jerarquía normal y $\Delta \chi^{2}<3.2$ para jerarquía inversa. El último desconocido es la jerarquía de masas de neutrinos (si $m_{1}<m_{2}<m_{3}$ (normal) o $m_{3}<m_{1}<m_{2}$ 

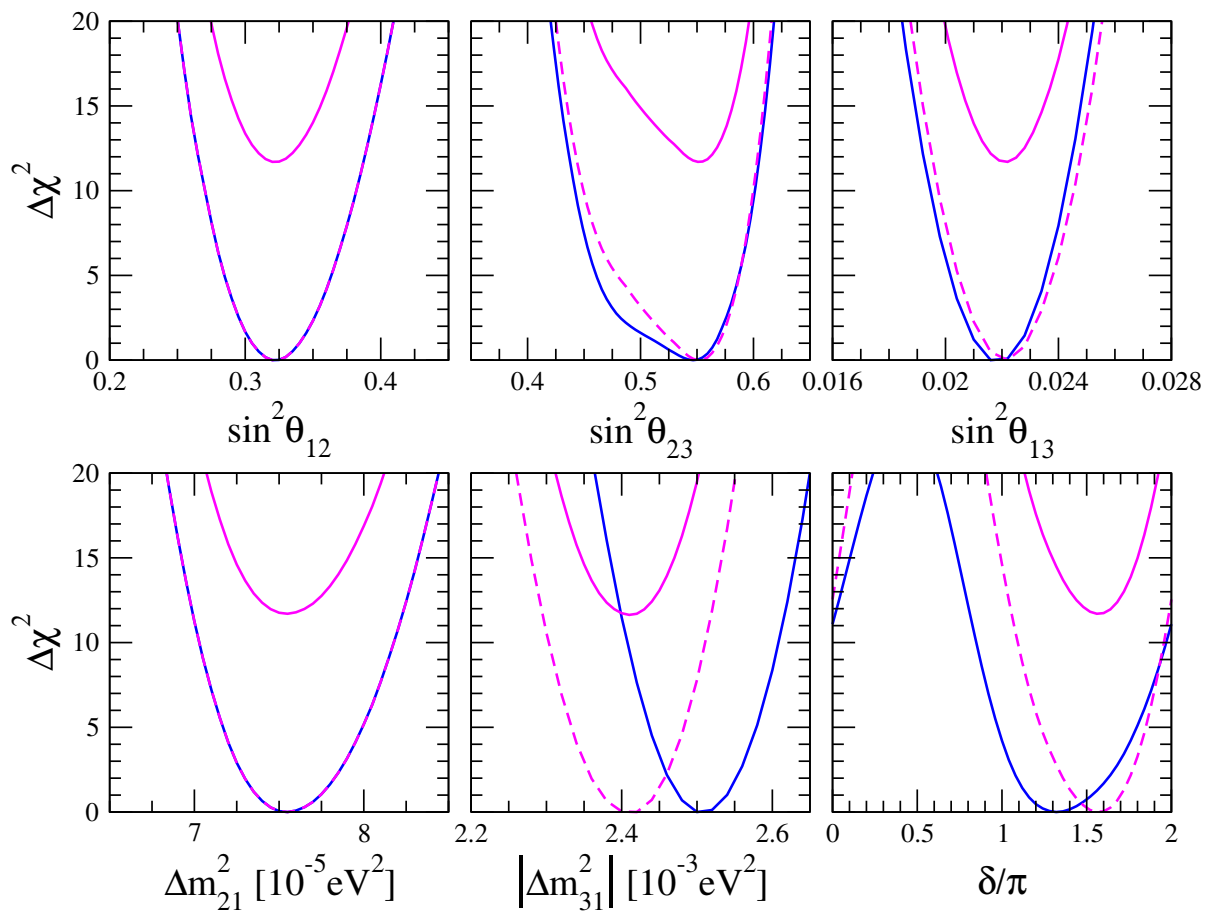

Figura 1: Los perfiles de los parámetros de oscilación obtenidos en el análisis global. Las líneas azules (magenta) corresponden al caso de jerarquía normal (inversa). Figura tomada de referencia [2].

(inversa)). En nuestro análisis global obtenemos por primera vez una preferencia de $3.4 \sigma$ en favor de la jerarquía normal. Parte de esta preferencia viene del experimento Super-K, que obtiene $\Delta \chi^{2}=3.5$ en favor de esta última. Por otro lado, hay un desacuerdo entre los experimentos de aceleradores y de reactores en la medida de $\theta_{13}$. Este desacuerdo es más pronunciado para la jerarquía inversa, lo cual resulta en una penalización más grande para esta jerarquía. La combinación de aceleradores y reactores da una preferencia de $\Delta \chi^{2}=7.5$, mientras el análisis de aceleradores solo da $\Delta \chi^{2}=2.0$.

\section{Los neutrinos y la violación de la simetría CPT}

Los resultados de estos capítulos fueron obtenidos en el paradigma de tres neutrinos. En el capítulo 3 , hemos discutido por primera vez un escenario más allá del modelo estándar. Dado que el teorema de CPT es una de las predicciones más 
sólidas de la teoría cuántica de campos relativista, una violación de esta simetría resultaría en un impacto enorme en la física de partículas. Usando datos de oscilación de neutrinos, podemos acotar observables de violación de la simetría CPT en el sector de los neutrinos. Para esto analizamos los datos de neutrinos y los de antineutrinos por separado, haciendo básicamente un análisis global de datos de neutrinos y un análisis global de datos de antineutrinos. Asumimos que las oscilaciones de neutrinos están descritas por los parámetros $\theta_{12}, \theta_{13}, \theta_{23}, \Delta m_{21}^{2}, \Delta m_{31}^{2}, \delta$, mientras las de los antineutrinos por $\bar{\theta}_{12}, \bar{\theta}_{13}, \bar{\theta}_{23}, \Delta \bar{m}_{21}^{2}, \Delta \bar{m}_{31}^{2}, \bar{\delta}$. Podemos usar los mismos datos como en el caso anterior, solo que tenemos que excluir los datos de experimentos atmosféricos, ya que no permiten distinguir entre eventos creados por neutrinos y antineutrinos. Entonces, podemos medir los parámetros de oscilación usando los siguientes conjuntos de datos:

- datos de neutrinos solares: $\theta_{12}, \Delta m_{21}^{2}, \theta_{13}$

- datos de neutrinos de experimentos de aceleradores K2K, MINOS, T2K y $\mathrm{NO} \nu \mathrm{A}: \theta_{23}, \Delta m_{31}^{2}, \theta_{13}$

- datos del experimento de reactor KamLAND: $\bar{\theta}_{12}, \Delta \bar{m}_{21}^{2}, \bar{\theta}_{13}$

- datos de los experimentos de reactor Daya Bay, RENO y Double Chooz: $\bar{\theta}_{13}, \Delta \bar{m}_{31}^{2}$

- datos de antineutrinos de experimentos de aceleradores K2K, MINOS, T2K y NO $\nu \mathrm{A}: 1]$ MINOS y T2K: $\bar{\theta}_{23}, \Delta \bar{m}_{31}^{2} \bar{\theta}_{13}$

Por el momento, no hay suficiente sensibilidad para medir los valores de $\delta$ y $\bar{\delta}$. Según el teorema de CPT, los dos análisis deberían coincidir. Es decir, los parámetros obtenidos tendrían que ser idénticos en los dos casos. El resultado de este análisis está presentado en la figura 2. Con este resultado, podemos calcular los perfiles de los observables de violación de CPT. La definición de estos observables está dada por:

$$
\Delta x=|x-\bar{x}|,
$$

donde $x$ es cualquiera de los parámetros de oscilación. A estos observables les asignamos las funciones de $\chi^{2}$ :

$$
\chi^{2}(\Delta x)=\chi^{2}(|x-\bar{x}|)=\chi^{2}(x)+\chi^{2}(\bar{x}) .
$$

\footnotetext{
${ }^{1} \mathrm{El}$ experimento K2K tomó solo datos de neutrinos. El experimento $\mathrm{NO} \nu \mathrm{A}$ no había publicado sus resultados con antineutrinos cuando se hizo este análisis.
} 

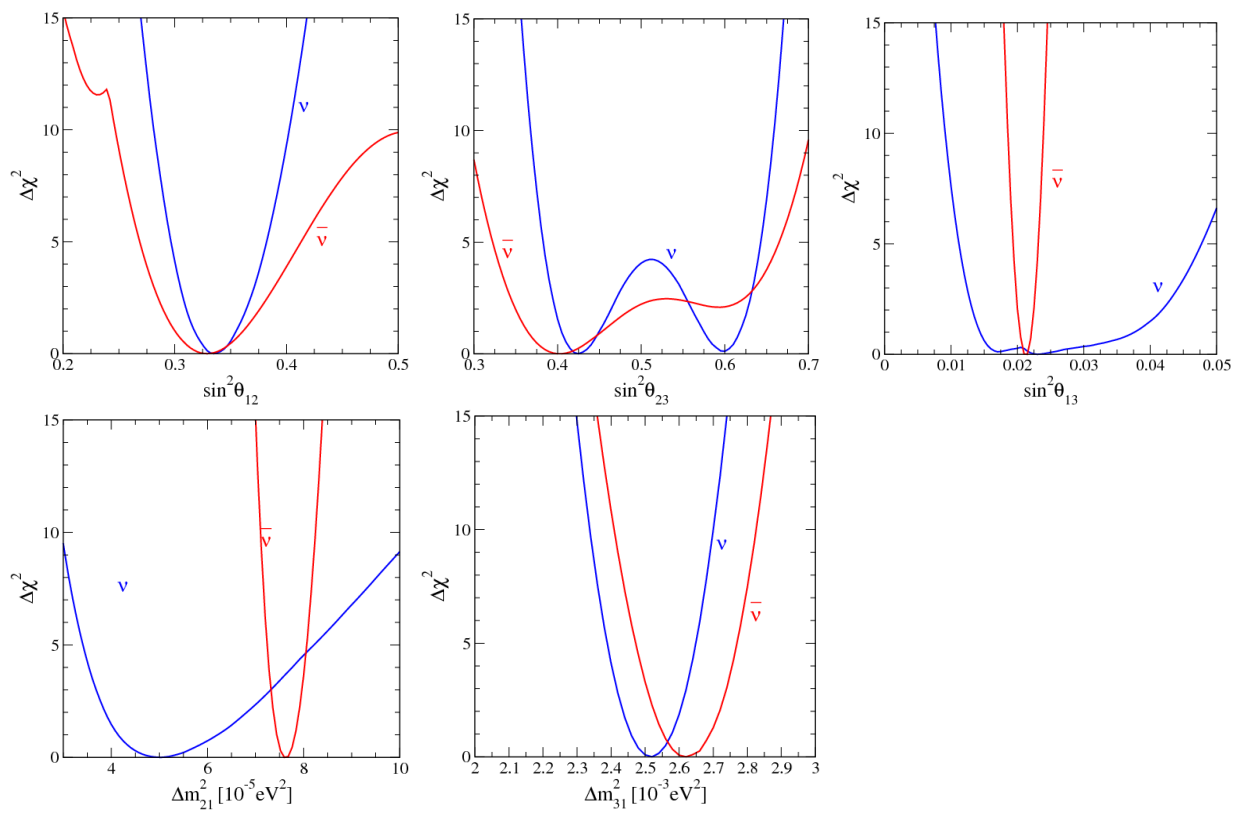

Figura 2: Los perfiles de los parámetros de oscilación obtenidos en un análisis global de datos de neutrinos (azul) y de antineutrinos (rojo).

Haciendo este análisis, obtenemos los resultados mostrados en la figura 3 . En números, estos perfiles se traducen en:

$$
\begin{aligned}
\left|\Delta m_{21}^{2}-\Delta \bar{m}_{21}^{2}\right| & <4.7 \times 10^{-5} \mathrm{eV}^{2} \\
\left|\Delta m_{31}^{2}-\Delta \bar{m}_{31}^{2}\right| & <3.7 \times 10^{-4} \mathrm{eV}^{2} \\
\left|\sin ^{2} \theta_{12}-\sin ^{2} \bar{\theta}_{12}\right| & <0.14 \\
\left|\sin ^{2} \theta_{13}-\sin ^{2} \bar{\theta}_{13}\right| & <0.03 \\
\left|\sin ^{2} \theta_{23}-\sin ^{2} \bar{\theta}_{23}\right| & <0.32
\end{aligned}
$$

a un nivel de confianza de $3 \sigma$. Estos resultados suponen las mejores cotas sobre violación de la simetría CPT en el sector de neutrinos hasta ahora.

\section{Oscilaciones de neutrinos en futuros experimentos}

En la segunda parte de la tesis hablamos de las capacidades de futuros experimentos, empezando en el capítulo 4 con una visión general de los futuros expe- 

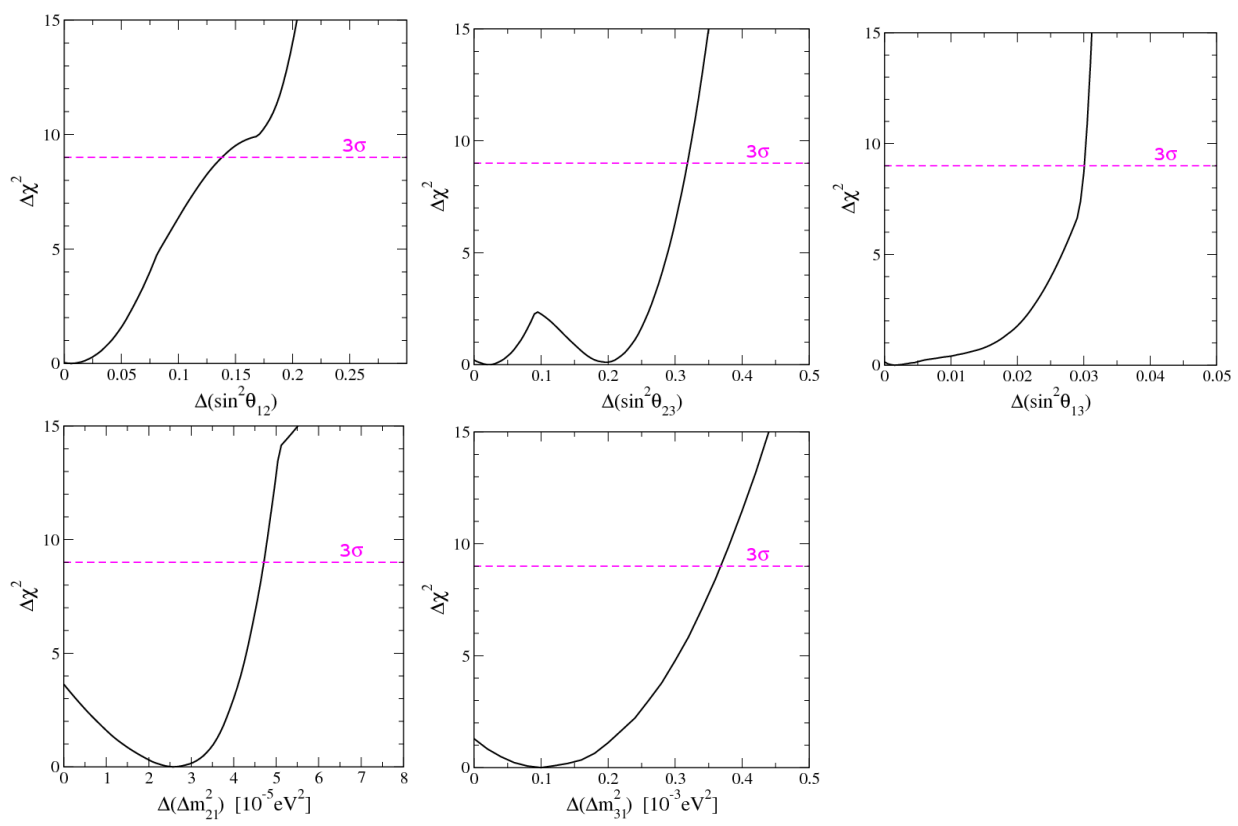

Figura 3: Los perfiles de $\chi^{2}$ de los observables de violación de CPT.

rimentos. Como veremos, los parámetros de oscilación se van a medir con mucha precisión. El futuro experimento de reactor JUNO va a medir los parámetros $\theta_{12} \mathrm{y}$ $\Delta m_{21}^{2}$ con un error de menos del 1\%. Los experimentos ORCA y PINGU medirán posiblemente el octante de $\theta_{23}$ y mejorarán la medida de $\Delta m_{31}^{2}$. Finalmente, los experimentos DUNE y T2HK van a poder medir la fase $\delta$ con muchísima precisión, además de contribuir a las medidas de $\Delta m_{31}^{2}$ y $\theta_{23}$. Además, todos estos experimentos van a poder medir la jerarquía de masas de los neutrinos. En el capítulo 5, nos enfocamos más en el caso de DUNE. Tras explicar cómo simular este futuro experimento de acelerador, discutimos la sensibilidad a los parámetros de oscilación. Tras 7 años tomando datos, DUNE mejorará considerablemente las medidas, como se aprecia en la figura 4. Desafortunadamente, la medida de $\theta_{13}$ en DUNE no va a ser tan buena como la medida actual en los experimentos de reactor. Como $\theta_{13}$ y $\theta_{23}$ están correlacionados, imponer un prior sobre $\theta_{13}$ afecta el análisis, como se ve en el panel central de la figura. Esta correlación es mucho menos fuerte en el caso de los otros dos parámetros como puede apreciarse.

En la segunda parte del capítulo 5 discutimos la posibilidad de medir la jerarquía de masas de neutrinos usando DUNE como detector de neutrinos atmosféricos. Para aumentar la sensibilidad, hemos propuesto usar la captura de muones 

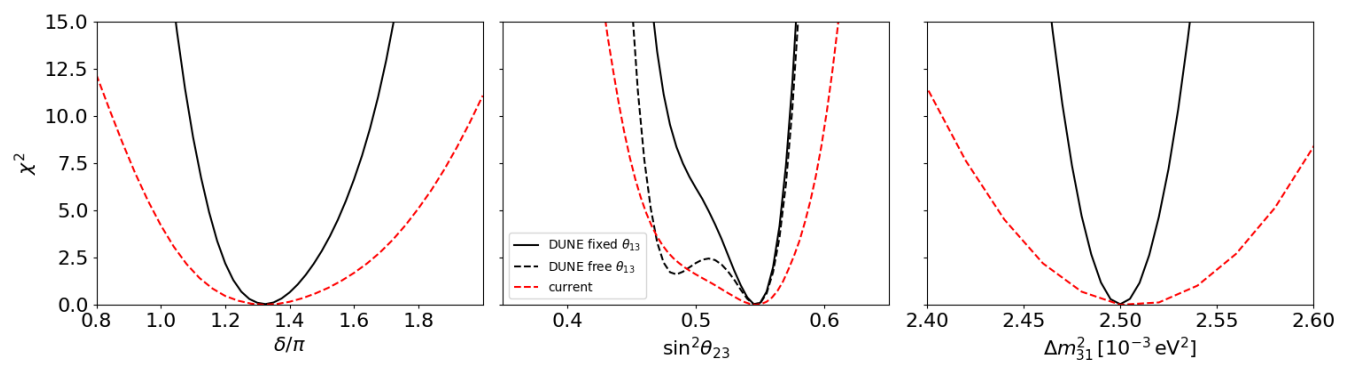

Figura 4: Los perfiles de $\chi^{2}$ para $\delta$ (izquierda), $\sin ^{2} \theta_{23}$ (centro) y $\Delta m_{31}^{2}$ (derecha). En el panel central mostramos como afecta $\theta_{13}$ a la determinación de $\theta_{23}$. La curva sólida corresponde al análisis con prior en $\theta_{13}$, mientras la curva discontinua refleja el análisis con $\theta_{13}$ libre. Para comparar, mostramos en rojo los perfiles actuales, obtenidos en el capítulo 2 .

en Argón. Cuando un neutrino muónico interactua con el Argón del detector de DUNE, se produce un muon. Este muon puede desintegrarse o puede ser capturado por el Argón. Esto pasa en un $(71.9 \pm 0.3) \%$ de los casos. Este proceso no es posible para antimuones. Por lo tanto, en un $72 \%$ de los casos aproximadamente se puede distinguir neutrinos de antineutrinos. Hemos simulado la señal atmosférica en DUNE después de 7 años de toma de datos y hemos hecho el análisis para obtener información sobre la jerarquía de masas de los neutrinos. Para comparar, se ha considerado un análisis con y un análisis sin la captura de muones en Argón. El resultado está presentado en la figura 5, en función del valor verdadero de $\theta_{23}$.

\section{La violación de CPT en DUNE}

En el capítulo 6 retomamos la discusión iniciada en el capítulo 3 en el contexto de DUNE. Hemos mostrado que DUNE podrá mejorar varias de las cotas obtenidas en el capítulo 3. Además de esto, hemos estudiado varios aspectos de la violación de CPT. En análisis como el presentado en el capítulo 2 se asume que la simetría CPT está conservada. Entonces, se analiza de forma conjunta los datos de neutrinos y antineutrinos. Sin embargo, si CPT fuera violada en la naturaleza, esto produciría errores en el análisis dando lugar a en soluciones falsas, que hemos llamado soluciones impostor. Hemos creado un conjunto de datos asumiendo diferentes ángulos de mezcla para neutrinos y antineutrinos. En el caso de neutrinos, tenemos $\sin ^{2} \theta_{23}=0.5 \mathrm{y}$, en el caso de antineutrinos, 


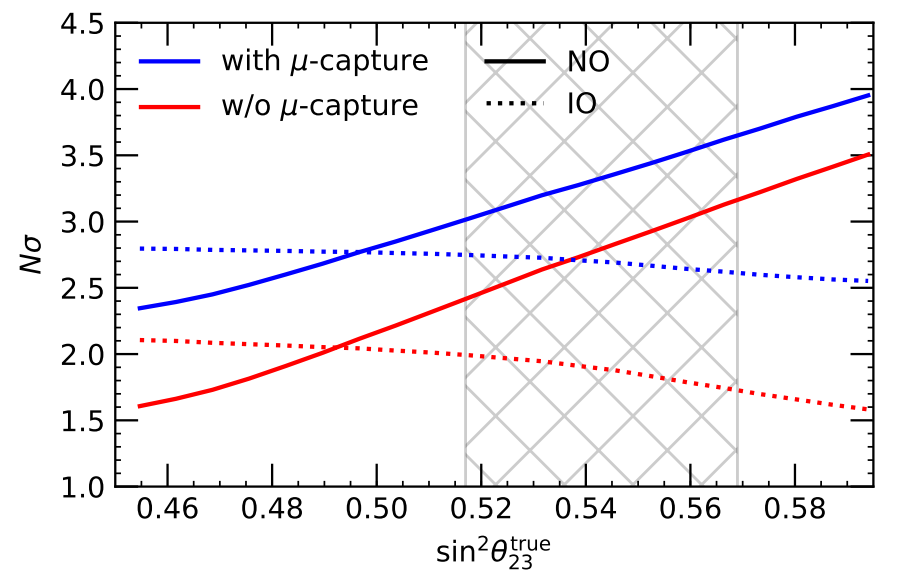

Figura 5: Sensibilidad de DUNE para medir la jerarquía de masas de los neutrinos en función de $\sin ^{2} \theta_{23}^{\text {true }}$. Las líneas azules (rojas) corresponden al análisis con ( $\sin$ ) usar la captura de muones en Argón. Las líneas sólidas asumen jerarquía normal como verdadera, mientras que las líneas discontinuas corresponden a la jerarquía inversa como verdadera. La banda gris corresponde al intervalo actual de la medida de $\sin ^{2} \theta_{23}$ a $1 \sigma$. Figura tomada de referencia [10].

$\sin ^{2} \theta_{23}=0.43$. El resto de parámetros son idénticos para ambos casos. Después hemos analizado estos datos asumiendo que CPT se conserva. El resultado está mostrado en la figura 6. Como se ve en la figura, obtenemos una nueva solución para $\sin ^{2} \theta_{23}^{\text {comb }}=0.467$. Los valores que asumimos como verdaderos quedan desfavorecidos a $3 \sigma$ y $6 \sigma$ aproximadamente.

Estos valores son cercanos a los medidos en el experimento T2K en un análisis separando neutrinos y antineutrinos. Los valores que se midieron son $\Delta m_{31}^{2}=$ $2.60 \times 10^{-3} \mathrm{eV}^{2}$ y $\sin ^{2} \theta_{23}=0.51$ en neutrinos y $\Delta \bar{m}_{31}^{2}=2.62 \times 10^{-3} \mathrm{eV}^{2}$ y $\sin ^{2} \bar{\theta}_{23}=0.42$ en antineutrinos. En el caso de T2K, las incertidumbres eran muy grandes de forma que el análisis no da indicaciones sobre una posible violación de CPT. En nuestro trabajo hemos investigado que ocurre si estos valores son verdaderos y hemos hecho el análisis en DUNE simulando los eventos esperados con estos valores de los parámetros. El resultado está presentado en la figura 7. Se ve claramente que las regiones están separadas. En este caso, el resultado sería una indicación de una violación de CPT. En la última parte de este capítulo, hemos estudiado si este resultado se podría confundir con alguna otra nueva física. Hemos encontrado que, efectivamente, es posible reproducir una señal como la estudiada con interacciones no estándar de los neutrinos (NSI, de "non-standard 


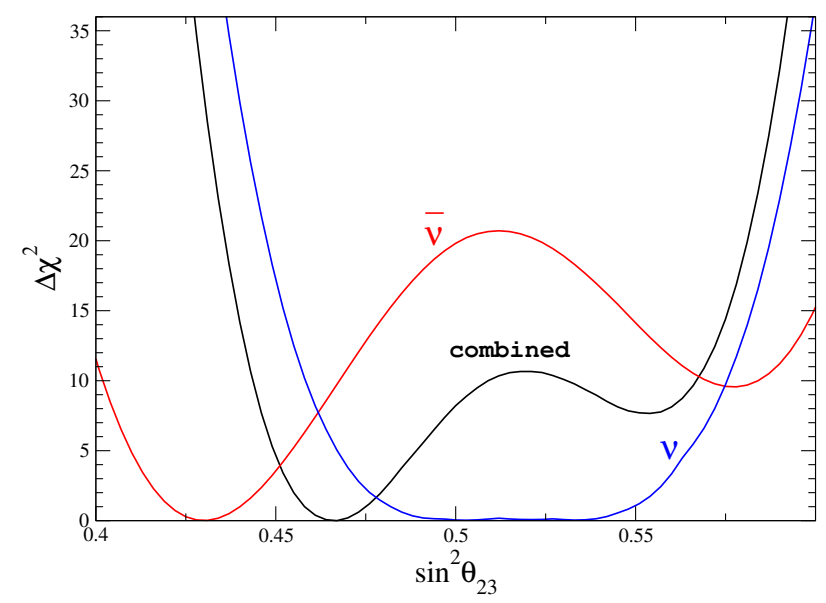

Figura 6: Sensibilidad de DUNE a la medida de $\sin ^{2} \theta_{23}$ para neutrinos (azul), antineutrinos (rojo) y la combinación de los dos bajo la hipótesis de conservación de la simetría CPT (negro). Figura tomada de referencia [5].
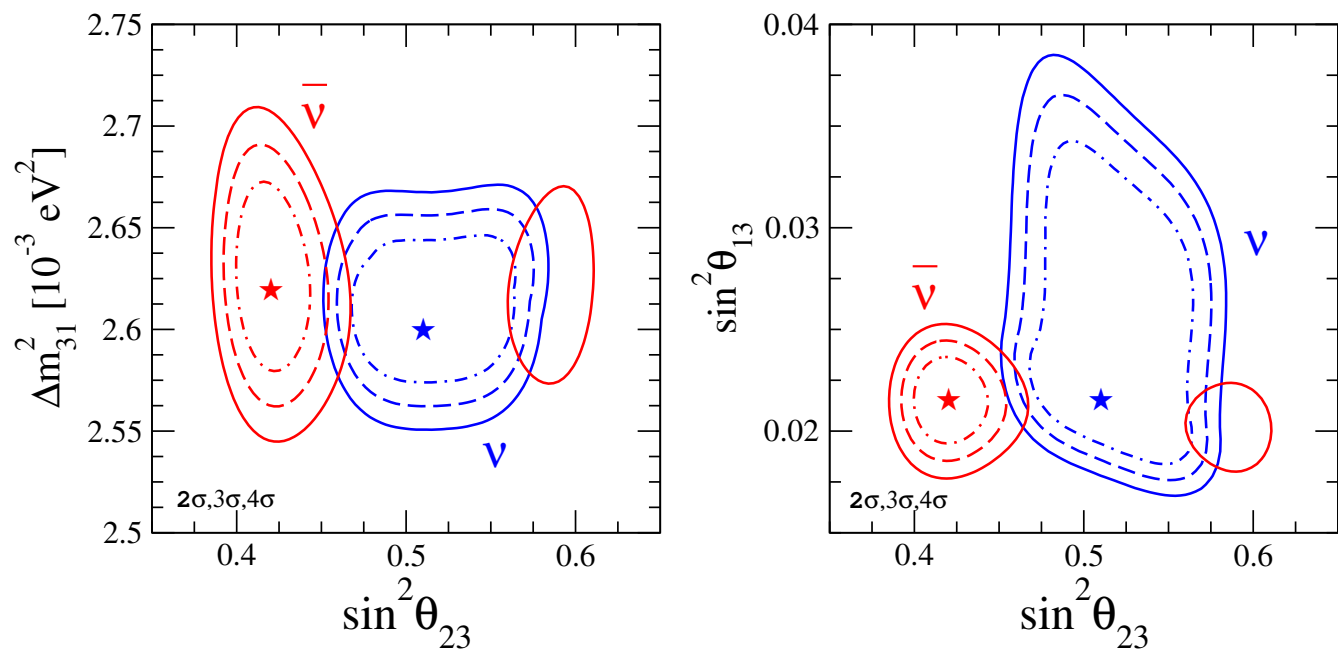

Figura 7: Regiones esperadas en DUNE para $2 \sigma, 3 \sigma$ y $4 \sigma$ en el plano $\sin ^{2} \theta_{23}-$ $\Delta m_{31}^{2}$ (izquierda) y $\sin ^{2} \theta_{23}-\sin ^{2} \theta_{13}$ (derecha) para neutrinos (azul) y antineutrinos (rojo). Figura tomada de referencia [5].

interactions"). En la figura 8 vemos que los espectros de eventos para el caso de CPT y el caso de NSI son idénticos. Sin embargo, para crear el espectro de NSI se necesita considerar otros parámetros más en el análisis. Uno de estos parámetros 

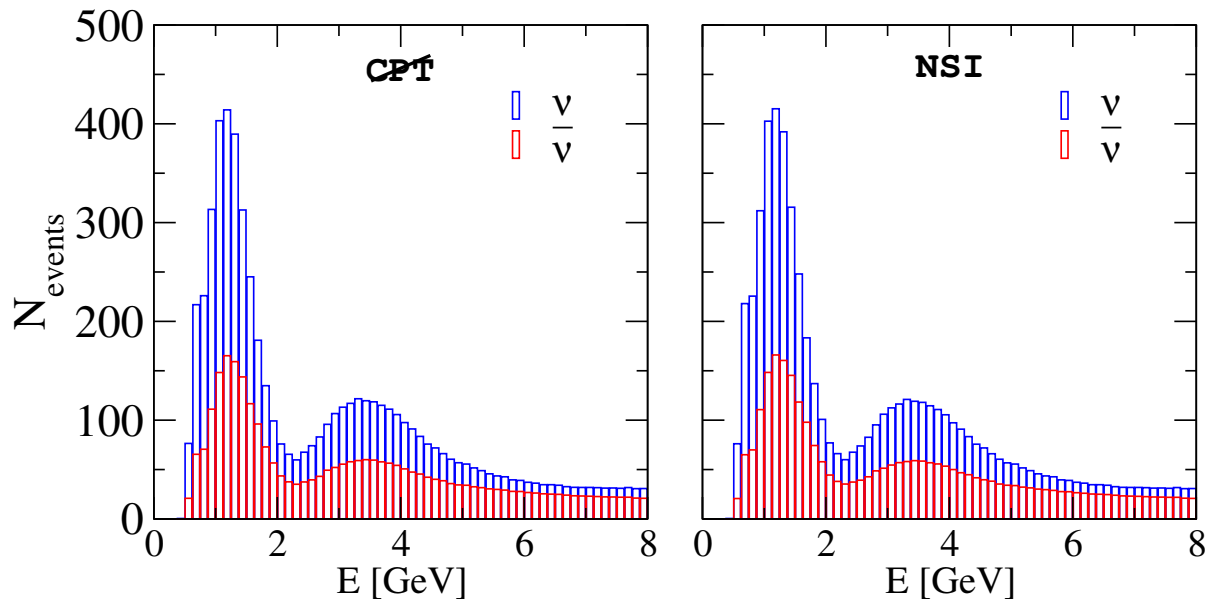

Figura 8: Número de eventos de neutrinos muónicos (azul) y antineutrinos muónicos (rojo) en el caso de violación de CPT (izquierda) y de conservación de CPT con NSI (derecha). Figura tomada de referencia 13.

tendría que tener un valor muy grande, que está excluido ya por experimentos actuales. Por lo tanto, una observación de este tipo sería verdaderamente una señal de violación de CPT.

\section{Oscilaciones de neutrinos quasi-Dirac en DUNE y JUNO}

Los experimentos de oscilaciones no pueden distinguir si los neutrinos son partículas de Dirac o partículas de Majorana. Generalmente, el caso de Dirac con $n$ neutrinos es un caso limite del caso de Majorana con $2 n$ neutrinos. Este límite se obtiene poniendo a cero los términos de masa de Majorana en el lagrangiano. Es posible obtener neutrinos de tipo quasi-Dirac si ambos términos, el de Majorana y el de Dirac, están presentes en el lagrangiano con una masa de Majorana muy pequeña. En este caso, aparecen nuevos ángulos y nuevas diferencias de masas en al análisis, lo cual puede afectar las oscilaciones de neutrinos. En el capítulo 7, hemos estudiado la sensibilidad de los experimentos DUNE y JUNO a este escenario con los 3 neutrinos estándar y sus 3 parejas de tipo quasiDirac. Como en este caso tenemos seis neutrinos, la matriz de mezcla se extiende a una matriz $6 \times 6$, con muchos ángulos y fases nuevas. Debido a la presencia de estos ángulos nuevos, una determinación de los parámetros de oscilación se hace 
básicamente imposible, porque cada ángulo estándar puede estar degenerado con uno o más ángulos nuevos. Por este motivo, definimos los nuevos observables

$$
\begin{aligned}
& X_{1}=\left|\mathbf{U}_{e 3}\right|^{2}+\left|\mathbf{U}_{e 6}\right|^{2}, X_{2}=\left|\mathbf{U}_{e 2}\right|^{2}+\left|\mathbf{U}_{e 5}\right|^{2}, \\
& X_{3}=\left|\mathbf{U}_{\mu 3}\right|^{2}+\left|\mathbf{U}_{\mu 6}\right|^{2}, X_{4}=\left|\mathbf{U}_{\mu 2}\right|^{2}+\left|\mathbf{U}_{\mu 5}\right|^{2}, \\
& X_{5}=\left|\mathbf{U}_{e 3} \mathbf{U}_{\mu 3}^{*}+\mathbf{U}_{e 6} \mathbf{U}_{\mu 6}^{*}\right|^{2}, X_{6}=\left|\mathbf{U}_{e 2} \mathbf{U}_{\mu 2}^{*}+\mathbf{U}_{e 5} \mathbf{U}_{\mu 5}^{*}\right|^{2}, \\
& X_{7}=\left(\mathbf{U}_{e 3} \mathbf{U}_{\mu 3}^{*}+\mathbf{U}_{e 6} \mathbf{U}_{\mu 6}^{*}\right)\left(\mathbf{U}_{e 2} \mathbf{U}_{\mu 2}^{*}+\mathbf{U}_{e 5} \mathbf{U}_{\mu 5}^{*}\right),
\end{aligned}
$$

que son funciones de todos los ángulos de mezcla. Las probabilidades de oscilación se pueden escribir en función de estos nuevos observables según

$$
\begin{aligned}
P\left(\nu_{e} \rightarrow \nu_{e}\right) & =1+\left(1-X_{1}-X_{2}\right) X_{2} \mathcal{A}_{21} \\
& +\left(1-X_{1}-X_{2}\right) X_{1} \mathcal{A}_{31}+X_{1} X_{2} \mathcal{A}_{32} \\
P\left(\nu_{\mu} \rightarrow \nu_{\mu}\right) & =1+\left(1-X_{3}-X_{4}\right) X_{4} \mathcal{A}_{21} \\
& +\left(1-X_{3}-X_{4}\right) X_{3} \mathcal{A}_{31}+X_{3} X_{4} \mathcal{A}_{32} \\
P\left(\nu_{e} \rightarrow \nu_{\mu}\right) & =-\left(X_{6}+\operatorname{Re} X_{7}\right) \mathcal{A}_{21}-\left(X_{5}+\operatorname{Re} X_{7}\right) \mathcal{A}_{31} \\
& +\operatorname{Re} X_{7} \mathcal{A}_{32}+\operatorname{Im} X_{7}\left(\mathcal{B}_{21}-\mathcal{B}_{31}+\mathcal{B}_{32}\right)
\end{aligned}
$$

donde $\mathcal{A}_{i j} \equiv-4 \sin ^{2}\left[\left(m_{i}^{2}-m_{j}^{2}\right) L /(4 E)\right]$ y $\mathcal{B}_{i j} \equiv 2 \sin \left[\left(m_{i}^{2}-m_{j}^{2}\right) L /(2 E)\right]$. En nuestro análisis incluimos un prior procedente de la medida de Daya Bay, que en este caso no es sobre $\theta_{13}$, sino sobre $\left(1-X_{1}\right) X_{1}$. El resultado de nuestro análisis combinado de DUNE y JUNO está mostrado en la figura 9. El panel izquierdo muestra la sensibilidad para determinar cada uno de los observables $X_{i}$. El panel derecho muestra la sensibilidad para medir la combinación $1-X_{5} / X_{1} X_{3}$. En el caso de oscilaciones estándar esta cantidad debe ser igual a cero. De nuestros resultados se deduce que DUNE y JUNO van a poder mejorar las medidas actuales considerablemente.

\section{La desintegración invisible de neutrinos en ORCA}

En el último capítulo de la tesis estudiamos el caso de neutrinos no estables. Una desintegración de neutrinos afectaría la probabilidad de oscilación y, por tanto, el número de eventos en un experimento. Nosotros estudiamos el caso donde un neutrino $\nu_{i}$ se desintegra en un neutrino más ligero $\nu_{j}$ y un nuevo bosón, el Majoron $J$, según $\nu_{i} \rightarrow \nu_{j}+J$ o $\nu_{i} \rightarrow \bar{\nu}_{j}+J$. Si el neutrino ligero 

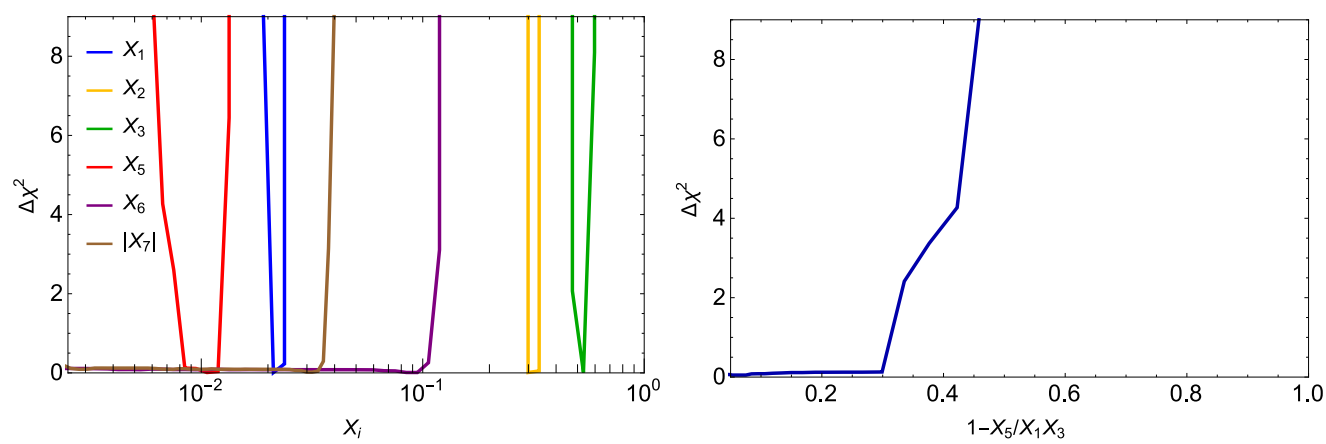

Figura 9: Panel izquierdo: Perfiles de $\chi^{2}$ para los observables $X_{i}$. Panel derecho: Perfil del observable $1-X_{5} / X_{1} X_{3}$, que en el caso de oscilaciones estándar debe ser cero. Figura tomada de referencia [15].

$\nu_{j}$ es un neutrino activo, se habla de desintegración visible mientras que, cuando se trata de un neutrino estéril se trataría de una desintegración invisible. Aquí nos enfocamos solamente en la desintegración invisible $\nu_{3} \rightarrow \nu_{4}+J$. Además asumimos que el neutrino estéril $\nu_{4}$ no se mezcla con los neutrinos activos. De esta forma los neutrinos se mezclan según

$$
\left(\begin{array}{l}
\nu_{\alpha} \\
\nu_{s}
\end{array}\right)=\left(\begin{array}{ll}
U & 0 \\
0 & 1
\end{array}\right)\left(\begin{array}{l}
\nu_{k} \\
\nu_{4}
\end{array}\right),
$$

donde $U$ es la matriz de mezcla estándar de tres neutrinos. Para tener en cuenta la desintegración de $\nu_{3}$ hay que adaptar el Hamiltoniano

$$
H=\frac{1}{2 E}\left[H_{0}+H_{m}+H_{D}\right],
$$

donde los primeros dos términos corresponden a las oscilaciones estándar en vacío y a los efectos de materia

$$
H_{0}=U\left(\begin{array}{ccc}
0 & 0 & 0 \\
0 & \Delta m_{21}^{2} & 0 \\
0 & 0 & \Delta m_{31}^{2}
\end{array}\right) U^{\dagger}, \quad H_{m}=\left(\begin{array}{ccc}
V & 0 & 0 \\
0 & 0 & 0 \\
0 & 0 & 0
\end{array}\right),
$$



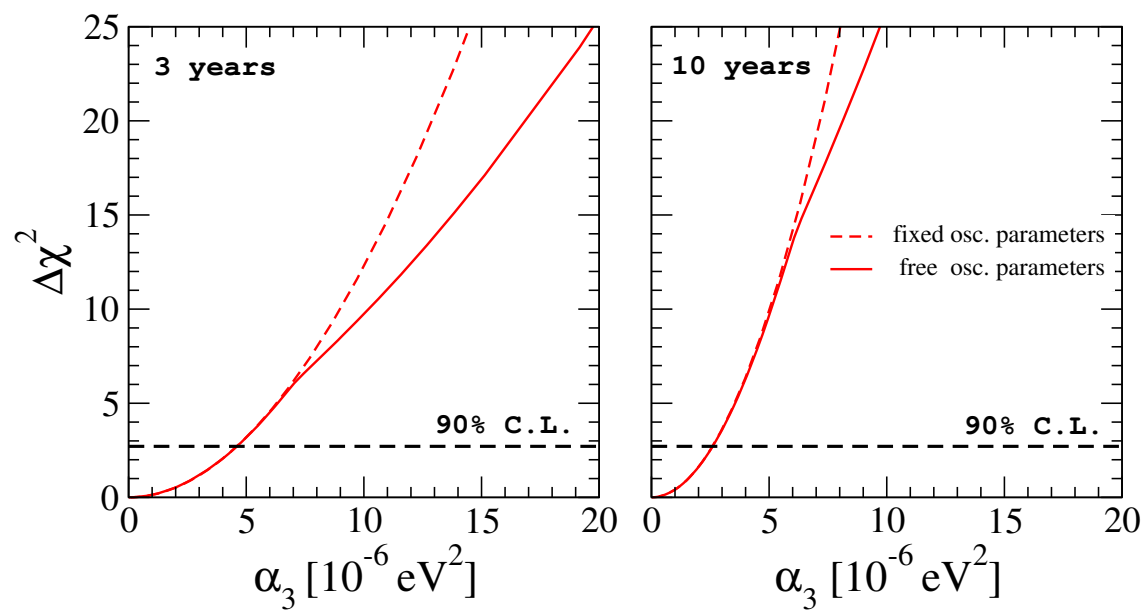

Figura 10: Sensibilidad de ORCA al parámetro $\alpha_{3}$. Las líneas sólidas (discontinuas) corresponden al análisis con $\Delta m_{31}^{2}$ y $\theta_{23}$ libre (fijado). El panel izquierdo (derecho) corresponde a tres (diez) años de datos. Figura tomada de referencia $[16$.

con $V=2 E \sqrt{2} G_{F} N_{e}$. $E$ es la energía del neutrino, $G_{F}$ la constante de Fermi y $N_{e}$ la densidad de electrones. El último termino incorpora la desintegración,

$$
H_{D}=U\left(\begin{array}{ccc}
0 & 0 & 0 \\
0 & 0 & 0 \\
0 & 0 & -i \alpha_{3}
\end{array}\right) U^{\dagger}
$$

En el capítulo 8 hemos estudiado la sensibilidad del futuro telescopio de neutrinos ORCA a acotar el parámetro $\alpha_{3}=m_{3} / \tau_{3}$ y cómo esta desintegración podría afectar la sensibilidad a los parámetros estándar. Para el análisis hemos asumido 3 y 10 años de datos. Se ha hecho un análisis teniendo en cuenta la variación de los parámetros de oscilación estándar y otro fijándolos a sus valores de mejor ajuste. La sensibilidad obtenida está mostrada en la figura 10 y la tabla 2 . Curiosamente, hemos encontrado que la desintegración de $\nu_{3}$ no afecta a la sensibilidad de medir los parámetros de oscilación estándar, como se ve en la figura 11. Además, hemos encontrado que la desintegración tampoco afectaría a la sensibilidad de ORCA a la medida de la jerarquía de masas de los neutrinos, uno de los principales objetivos del experimento. 


\begin{tabular}{|c|c|c|}
\hline tiempo & $\alpha_{3}\left[\mathrm{eV}^{2}\right]$ & $\tau_{3} / m_{3}[\mathrm{~s} / \mathrm{eV}]$ \\
\hline 3 años & $<4.6 \times 10^{-6}$ & $>1.4 \times 10^{-10}$ \\
\hline 10 años & $<2.6 \times 10^{-6}$ & $>2.5 \times 10^{-10}$ \\
\hline
\end{tabular}

Tabla 2: Cotas esperadas (90\% C.L.) tras 3 y 10 años de toma de datos en ORCA.
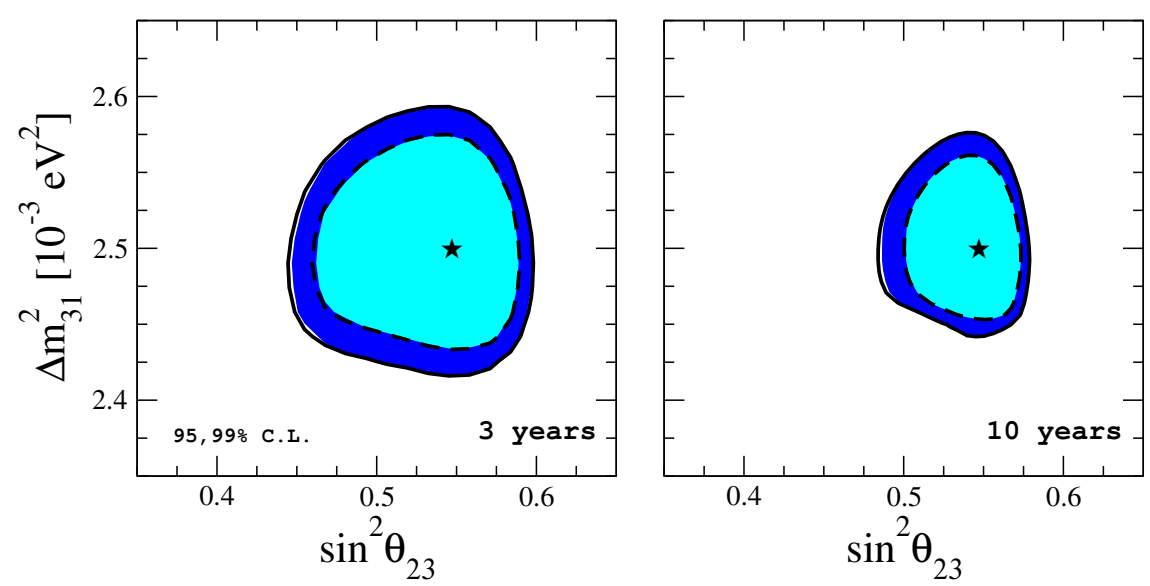

Figura 11: Sensibilidad de ORCA a medir los parámetros de oscilación en el caso de tres neutrinos estables (regiones coloreadas) e incluyendo la desintegración de $\nu_{3}$ (líneas negras) a $95 \%$ y $99 \%$ C.L., después de 3 años (izquierda) y 10 años (derecha) de datos. Figura tomada de referencia [16. 


\section{Bibliography}

[1] G. Barenboim, P. B. Denton, S. J. Parke, C. A. Ternes, Neutrino Oscillation Probabilities through the Looking Glass, Phys. Lett. B791 (2019) 351-360. arXiv:1902.00517, doi:10.1016/j.physletb.2019.03.002

[2] P. F. de Salas, D. V. Forero, C. A. Ternes, M. Tortola, J. W. F. Valle, Status of neutrino oscillations 2018: $3 \sigma$ hint for normal mass ordering and improved CP sensitivity, Phys. Lett. B782 (2018) 633-640. arXiv:1708. 01186, doi:10.1016/j.physletb.2018.06.019.

[3] S. Gariazzo, M. Archidiacono, P. F. de Salas, O. Mena, C. A. Ternes, M. Tortola, Neutrino masses and their ordering: Global Data, Priors and Models, JCAP 1803 (03) (2018) 011. arXiv:1801.04946, doi:10.1088/ $1475-7516 / 2018 / 03 / 011$

[4] P. F. De Salas, S. Gariazzo, O. Mena, C. A. Ternes, M. Tortola, Neutrino Mass Ordering from Oscillations and Beyond: 2018 Status and Future Prospects, Front. Astron. Space Sci. 5 (2018) 36. arXiv:1806.11051, doi:10.3389/fspas.2018.00036

[5] G. Barenboim, C. A. Ternes, M. Tortola, Neutrinos, DUNE and the world best bound on CPT invariance, Phys. Lett. B780 (2018) 631-637. arXiv: 1712.01714, doi:10.1016/j.physletb.2018.03.060.

[6] G. Barenboim, M. Tórtola, C. A. Ternes, CPT and CP, an entangled couplearXiv:2005.05975.

[7] S. Gariazzo, C. Giunti, C. A. Ternes, Status of 3+1 global fits of shortbaseline neutrino oscillations (preliminary).

[8] G. A. Barenboim, P. Martínez-Miravé, C. A. Ternes, M. A. Tórtola, Sterile neutrinos with altered dispersion relations revisited, JHEP 03 (2020) 070. arXiv: 1911.02329. 
[9] A. de Gouvea, V. de Romeri, C. A. Ternes, Probing neutrino quantum decoherence at reactor experimentsarXiv:2005.03022.

[10] C. A. Ternes, S. Gariazzo, R. Hajjar, O. Mena, M. Sorel, M. Tórtola, Neutrino Mass Ordering at DUNE: an Extra $\nu$-Bonus, Phys. Rev. D100 (2019) 093004. arXiv:1905.03589, doi:10.1103/PhysRevD.100.093004.

[11] R. Srivastava, C. A. Ternes, M. Tortola, J. W. F. Valle, Testing a lepton quarticity flavor theory of neutrino oscillations with the DUNE experiment, Phys. Lett. B778 (2018) 459-463. arXiv:1711.10318, doi:10.1016/j. physletb.2018.01.014

[12] R. Srivastava, C. A. Ternes, M. Tortola, J. W. F. Valle, Zooming in on neutrino oscillations with DUNE, Phys. Rev. D97 (9) (2018) 095025. arXiv:1803.10247, doi:10.1103/PhysRevD.97.095025.

[13] G. Barenboim, C. A. Ternes, M. Tórtola, New physics vs new paradigms: distinguishing CPT violation from NSI, Eur. Phys. J. C79 (5) (2019) 390. arXiv:1804.05842, doi:10.1140/epjc/s10052-019-6900-7.

[14] G. Barenboim, M. Masud, C. A. Ternes, M. Tortola, Exploring the intrinsic Lorentz-violating parameters at DUNE, Phys. Lett. B788 (2019) 308-315. arXiv:1805.11094, doi:10.1016/j.physletb.2018.11.040

[15] G. Anamiati, V. De Romeri, M. Hirsch, C. A. Ternes, M. Tórtola, QuasiDirac neutrino oscillations at DUNE and JUNO, Phys. Rev. D100 (3) (2019) 035032. arXiv:1907.00980, doi:10.1103/PhysRevD.100.035032.

[16] P. F. de Salas, S. Pastor, C. A. Ternes, T. Thakore, M. Tórtola, Constraining the invisible neutrino decay with KM3NeT-ORCA, Phys. Lett. B789 (2019) 472-479. arXiv:1810.10916, doi:10.1016/j.physletb.2018.12. 066 .

[17] B. Pontecorvo, Mesonium and anti-mesonium, Sov. Phys. JETP 6 (1957) 429, [Zh. Eksp. Teor. Fiz.33,549(1957)].

[18] B. Pontecorvo, Inverse beta processes and nonconservation of lepton charge, Sov. Phys. JETP 7 (1958) 172-173, [Zh. Eksp. Teor. Fiz.34,247(1957)].

[19] V. N. Gribov, B. Pontecorvo, Neutrino astronomy and lepton charge, Phys. Lett. 28B (1969) 493. doi:10.1016/0370-2693(69)90525-5. 
[20] Z. Maki, M. Nakagawa, S. Sakata, Remarks on the unified model of elementary particles, Prog. Theor. Phys. 28 (1962) 870-880. doi:10.1143/ PTP. 28.870.

[21] B. Pontecorvo, Neutrino Experiments and the Problem of Conservation of Leptonic Charge, Sov. Phys. JETP 26 (1968) 984-988, [Zh. Eksp. Teor. Fiz.53,1717(1967)].

[22] Y. Fukuda, et al., Evidence for oscillation of atmospheric neutrinos, Phys. Rev. Lett. 81 (1998) 1562-1567. arXiv:hep-ex/9807003, doi:10.1103/ PhysRevLett.81.1562.

[23] Q. R. Ahmad, et al., Direct evidence for neutrino flavor transformation from neutral current interactions in the Sudbury Neutrino Observatory, Phys. Rev. Lett. 89 (2002) 011301. arXiv:nucl-ex/0204008, doi:10. 1103/PhysRevLett.89.011301.

[24] A. B. McDonald, Nobel Lecture: The Sudbury Neutrino Observatory: Observation of flavor change for solar neutrinos, Rev. Mod. Phys. 88 (3) (2016) 030502. doi:10.1103/RevModPhys.88.030502.

[25] T. Kajita, Nobel Lecture: Discovery of atmospheric neutrino oscillations, Rev. Mod. Phys. 88 (3) (2016) 030501. doi:10.1103/RevModPhys.88. 030501 .

[26] C. Giunti, C. W. Kim, Fundamentals of Neutrino Physics and Astrophysics, 2007.

[27] L. Wolfenstein, Neutrino Oscillations in Matter, Phys. Rev. D17 (1978) 2369-2374. doi:10.1103/PhysRevD.17.2369.

[28] V. D. Barger, K. Whisnant, S. Pakvasa, R. J. N. Phillips, Matter Effects on Three-Neutrino Oscillations, Phys. Rev. D22 (1980) 2718. doi:10.1103/ PhysRevD.22.2718.

[29] S. P. Mikheyev, A. Yu. Smirnov, Resonance Amplification of Oscillations in Matter and Spectroscopy of Solar Neutrinos, Sov. J. Nucl. Phys. 42 (1985) 913-917.

[30] S. P. Mikheev, A. Yu. Smirnov, Resonant amplification of neutrino oscillations in matter and solar neutrino spectroscopy, Nuovo Cim. C9 (1986) 17-26. doi: 10.1007/BF02508049. 
[31] H. W. Zaglauer, K. H. Schwarzer, The Mixing Angles in Matter for Three Generations of Neutrinos and the Msw Mechanism, Z. Phys. C40 (1988) 273. doi:10.1007/BF01555889.

[32] J. Arafune, M. Koike, J. Sato, CP violation and matter effect in long baseline neutrino oscillation experiments, Phys. Rev. D56 (1997) 30933099, [Erratum: Phys. Rev.D60,119905(1999)]. arXiv:hep-ph/9703351, doi:10.1103/PhysRevD.60.119905,10.1103/PhysRevD.56.3093.

[33] A. Cervera, A. Donini, M. B. Gavela, J. J. Gomez Cadenas, P. Hernandez, O. Mena, S. Rigolin, Golden measurements at a neutrino factory, Nucl. Phys. B579 (2000) 17-55, [Erratum: Nucl. Phys.B593,731(2001)]. arXiv:hep-ph/0002108, doi:10.1016/ S0550-3213(00)00606-4,10.1016/S0550-3213(00)00221-2.

[34] K. Asano, H. Minakata, Large-Theta(13) Perturbation Theory of Neutrino Oscillation for Long-Baseline Experiments, JHEP 06 (2011) 022. arXiv: 1103.4387, doi:10.1007/JHEP06(2011) 022 .

[35] M. Freund, Analytic approximations for three neutrino oscillation parameters and probabilities in matter, Phys. Rev. D64 (2001) 053003. arXiv: hep-ph/0103300, doi:10.1103/PhysRevD.64.053003.

[36] E. K. Akhmedov, R. Johansson, M. Lindner, T. Ohlsson, T. Schwetz, Series expansions for three flavor neutrino oscillation probabilities in matter, JHEP 04 (2004) 078. arXiv:hep-ph/0402175, doi:10.1088/1126-6708/ $2004 / 04 / 078$.

[37] A. Friedland, C. Lunardini, Two modes of searching for new neutrino interactions at MINOS, Phys. Rev. D74 (2006) 033012. arXiv: hep-ph/0606101, doi:10.1103/PhysRevD.74.033012.

[38] H. Minakata, S. J. Parke, Simple and Compact Expressions for Neutrino Oscillation Probabilities in Matter, JHEP 01 (2016) 180. arXiv:1505. 01826, doi:10.1007/JHEP01(2016)180.

[39] P. B. Denton, H. Minakata, S. J. Parke, Compact Perturbative Expressions For Neutrino Oscillations in Matter, JHEP 06 (2016) 051. arXiv:1604. 08167, doi:10.1007/JHEP06(2016) 051.

[40] P. B. Denton, S. J. Parke, Addendum to "Compact perturbative expressions for neutrino oscillations in matter", JHEP 06 (2018) 109. arXiv:1801. 06514, doi:10.1007/JHEP06(2018) 109 . 
[41] H. Nunokawa, S. J. Parke, R. Zukanovich Funchal, Another possible way to determine the neutrino mass hierarchy, Phys. Rev. D72 (2005) 013009. arXiv:hep-ph/0503283, doi:10.1103/PhysRevD.72.013009.

[42] S. Parke, What is $\Delta m_{e e}^{2}$ ?, Phys. Rev. D93 (5) (2016) 053008. arXiv: 1601.07464, doi:10.1103/PhysRevD.93.053008.

[43] R. Acciarri, et al., Long-Baseline Neutrino Facility (LBNF) and Deep Underground Neutrino Experiment (DUNE)arXiv:1601.05471.

[44] K. J. Kelly, S. J. Parke, Matter Density Profile Shape Effects at DUNE, Phys. Rev. D98 (1) (2018) 015025. arXiv:1802.06784, doi:10.1103/ PhysRevD.98.015025.

[45] D. S. Ayres, et al., NOvA: Proposal to Build a 30 Kiloton Off-Axis Detector to Study $\nu_{\mu} \rightarrow \nu_{e}$ Oscillations in the NuMI BeamlinearXiv: hep-ex/0503053

[46] Y. Itow, et al., The JHF-Kamioka neutrino project, in: Neutrino oscillations and their origin. Proceedings, 3rd International Workshop, NOON 2001, Kashiwa, Tokyo, Japan, December 508, 2001, 2001, pp. 239-248. arXiv:hep-ex/0106019.

[47] K. Abe, et al., A Long Baseline Neutrino Oscillation Experiment Using JPARC Neutrino Beam and Hyper-Kamiokande, 2014. arXiv:1412.4673.

[48] K. Abe, et al., Physics potential of a long-baseline neutrino oscillation experiment using a J-PARC neutrino beam and Hyper-Kamiokande, PTEP 2015 (2015) 053C02. arXiv: 1502.05199, doi:10.1093/ptep/ptv061.

[49] E. Baussan, et al., A very intense neutrino super beam experiment for leptonic $\mathrm{CP}$ violation discovery based on the European spallation source linac, Nucl. Phys. B885 (2014) 127-149. arXiv:1309.7022, doi:10.1016/ j.nuclphysb.2014.05.016.

[50] C. Albright, et al., Physics at a neutrino factoryarXiv:hep-ex/0008064.

[51] S. J. Parke, Theta(13), in: Particles and nuclei : Seventeenth International Conference on Particles and Nuclei, Santa Fe, New Mexico, 23-30 October 2005, 2005.

URL http://lss.fnal.gov/cgi-bin/find_paper.pl?conf-05-603 
[52] S. J. Parke, Neutrinos: in and out of the standard model, AIP Conf. Proc. 885 (2007) 69-84. doi:10.1063/1.2563180.

[53] H. Nunokawa, S. J. Parke, J. W. F. Valle, CP Violation and Neutrino Oscillations, Prog. Part. Nucl. Phys. 60 (2008) 338-402. arXiv:0710.0554, doi:10.1016/j.ppnp.2007.10.001.

[54] H. Minakata, S. J. Parke, Correlated, precision measurements of $\theta_{23}$ and $\delta$ using only the electron neutrino appearance experiments, Phys. Rev. D87 (11) (2013) 113005. arXiv:1303.6178, doi:10.1103/PhysRevD.87. 113005 .

[55] S. K. Agarwalla, Y. Kao, T. Takeuchi, Analytical approximation of the neutrino oscillation matter effects at large $\theta_{13}$, JHEP 04 (2014) 047. arXiv: 1302.6773, doi:10.1007/JHEP04(2014) 047.

[56] P. B. Denton, S. J. Parke, The effective $\Delta m_{e e}^{2}$ in matter, Phys. Rev. D98 (9) (2018) 093001. arXiv:1808.09453, doi:10.1103/PhysRevD.98.093001

[57] P. B. Denton, S. J. Parke, X. Zhang, Rotations Versus Perturbative Expansions for Calculating Neutrino Oscillation Probabilities in Matter, Phys. Rev. D98 (3) (2018) 033001. arXiv:1806.01277, doi:10.1103/PhysRevD. 98.033001.

[58] J. Kopp, Efficient numerical diagonalization of hermitian $3 \times 3$ matrices, Int. J. Mod. Phys. C19 (2008) 523-548. arXiv:physics/0610206, doi: 10.1142/S0129183108012303.

[59] C. Argüelles, J. Salvado, C. Weaver, https://github.com/Arguelles/ nuSQuIDS

[60] G. Cardano, Ars Magna, 1545.

[61] Eigen 3.3.4, http://eigen.tuxfamily.org.

[62] T. Hahn, Routines for the diagonalization of complex matricesarXiv: physics/0607103.

[63] Feynarts, http://www.feynarts.de/diag/

[64] O. Yasuda, Vacuum mimicking phenomena in neutrino oscillations, Phys. Lett. B516 (2001) 111-115. arXiv:hep-ph/0106232, doi:10.1016/ S0370-2693(01)00920-0. 
[65] B. Abi, et al., The DUNE Far Detector Interim Design Report Volume 1: Physics, Technology and StrategiesarXiv:1807.10334.

[66] B. Abi, et al., The DUNE Far Detector Interim Design Report, Volume 2: Single-Phase ModulearXiv:1807.10327.

[67] B. Abi, et al., The DUNE Far Detector Interim Design Report, Volume 3: Dual-Phase ModulearXiv:1807.10340

[68] W. J. Marciano, Extra long baseline neutrino oscillations and CP violationarXiv:hep-ph/0108181.

[69] H. Minakata, H. Nunokawa, Exploring neutrino mixing with low-energy superbeams, JHEP 10 (2001) 001. arXiv:hep-ph/0108085, doi:10.1088/ $1126-6708 / 2001 / 10 / 001$.

[70] Y. Nakano, PhD Thesis, University of Tokyo, http://www-sk.icrr. u-tokyo.ac.jp/sk/_pdf/articles/2016/doc_thesis_naknao.pdf (2016).

[71] W. C. Haxton, R. G. Hamish Robertson, A. M. Serenelli, Solar Neutrinos: Status and Prospects, Ann. Rev. Astron. Astrophys. 51 (2013) 21-61. arXiv:1208.5723, doi:10.1146/annurev-astro-081811-125539.

[72] B. T. Cleveland, T. Daily, R. Davis, Jr., J. R. Distel, K. Lande, C. K. Lee, P. S. Wildenhain, J. Ullman, Measurement of the solar electron neutrino flux with the Homestake chlorine detector, Astrophys. J. 496 (1998) 505526. doi:10.1086/305343

[73] F. Kaether, W. Hampel, G. Heusser, J. Kiko, T. Kirsten, Reanalysis of the GALLEX solar neutrino flux and source experiments, Phys. Lett. B685 (2010) 47-54. arXiv:1001.2731, doi:10.1016/j.physletb.2010. 01.030 .

[74] J. N. Abdurashitov, et al., Measurement of the solar neutrino capture rate with gallium metal. III: Results for the 2002-2007 data-taking period, Phys. Rev. C80 (2009) 015807. arXiv:0901.2200, doi:10.1103/PhysRevC.80. 015807 .

[75] G. Bellini, et al., Precision measurement of the 7Be solar neutrino interaction rate in Borexino, Phys. Rev. Lett. 107 (2011) 141302. arXiv: 1104.1816, doi:10.1103/PhysRevLett.107.141302. 
[76] G. Bellini, et al., Final results of Borexino Phase-I on low energy solar neutrino spectroscopy, Phys. Rev. D89 (11) (2014) 112007. arXiv:1308. 0443, doi:10.1103/PhysRevD.89.112007.

[77] J. Hosaka, et al., Solar neutrino measurements in super-Kamiokande-I, Phys. Rev. D73 (2006) 112001. arXiv:hep-ex/0508053, doi:10.1103/ PhysRevD.73.112001.

[78] J. P. Cravens, et al., Solar neutrino measurements in Super-KamiokandeII, Phys. Rev. D78 (2008) 032002. arXiv:0803.4312, doi:10.1103/ PhysRevD.78.032002.

[79] K. Abe, et al., Solar neutrino results in Super-Kamiokande-III, Phys. Rev. D83 (2011) 052010. arXiv:1010.0118, doi:10.1103/PhysRevD.83. 052010.

[80] B. Aharmim, et al., An Independent Measurement of the Total Active B8 Solar Neutrino Flux Using an Array of He-3 Proportional Counters at the Sudbury Neutrino Observatory, Phys. Rev. Lett. 101 (2008) 111301. arXiv:0806.0989, doi:10.1103/PhysRevLett.101.111301.

[81] B. Aharmim, et al., Low Energy Threshold Analysis of the Phase I and Phase II Data Sets of the Sudbury Neutrino Observatory, Phys. Rev. C81 (2010) 055504. arXiv:0910.2984, doi:10.1103/PhysRevC.81.055504

[82] S. Abe, et al., Precision Measurement of Neutrino Oscillation Parameters with KamLAND, Phys. Rev. Lett. 100 (2008) 221803. arXiv:0801.4589, doi:10.1103/PhysRevLett.100.221803.

[83] G. Bak, et al., Measurement of Reactor Antineutrino Oscillation Amplitude and Frequency at RENO, Phys. Rev. Lett. 121 (20) (2018) 201801. arXiv: 1806.00248, doi:10.1103/PhysRevLett.121.201801.

[84] D. Adey, et al., Measurement of the Electron Antineutrino Oscillation with 1958 Days of Operation at Daya Bay, Phys. Rev. Lett. 121 (24) (2018) 241805. arXiv:1809.02261, doi:10.1103/PhysRevLett.121.241805.

[85] A. Hernandez-Cabezudo, S. J. Parke, S.-H. Seo, Constraint on the solar $\Delta m^{2}$ using 4000 days of short baseline reactor neutrino data, Phys. Rev. D 100 (11) (2019) 113008. arXiv:1905.09479, doi:10.1103/PhysRevD. 100.113008 . 
[86] F. P. An, et al., Measurement of electron antineutrino oscillation based on 1230 days of operation of the Daya Bay experiment, Phys. Rev. D95 (7) (2017) 072006. arXiv:1610.04802, doi:10.1103/PhysRevD.95.072006

[87] S.-H. Seo, New Results from RENO using 1500 Days of Data, in: 15th International Conference on Topics in Astroparticle and Underground Physics (TAUP 2017) Sudbury, Ontario, Canada, July 24-28, 2017, 2017. arXiv:1710.08204.

[88] M. Y. Pac, Recent Results from RENO, PoS NuFact2017 (2018) 038. arXiv: 1801.04049, doi:10.22323/1.295.0038.

[89] M. Ishitsuka, Double Chooz Reactor Antineutrino Experiment, https://indico.in2p3.fr/event/12279/session/3/contribution/ 173/material/slides/0.pdf (March 2016).

[90] J. K. Ahn, et al., RENO: An Experiment for Neutrino Oscillation Parameter $\theta_{13}$ Using Reactor Neutrinos at YonggwangarXiv:1003.1391.

[91] J. H. Choi, et al., Observation of Energy and Baseline Dependent Reactor Antineutrino Disappearance in the RENO Experiment, Phys. Rev. Lett. 116 (21) (2016) 211801. arXiv:1511.05849, doi:10.1103/PhysRevLett. 116.211801 .

[92] E. Vitagliano, I. Tamborra, G. Raffelt, Grand Unified Neutrino Spectrum at EartharXiv: 1910.11878.

[93] M. G. Aartsen, et al., Determining neutrino oscillation parameters from atmospheric muon neutrino disappearance with three years of IceCube DeepCore data, Phys. Rev. D91 (7) (2015) 072004. arXiv:1410.7227, doi:10.1103/PhysRevD.91.072004.

[94] S. Adrian-Martinez, et al., Measurement of Atmospheric Neutrino Oscillations with the ANTARES Neutrino Telescope, Phys. Lett. B714 (2012) 224-230. arXiv:1206.0645, doi:10.1016/j.physletb.2012.07.002.

[95] K. Abe, et al., Atmospheric neutrino oscillation analysis with external constraints in Super-Kamiokande I-IV, Phys. Rev. D97 (7) (2018) 072001. arXiv:1710.09126, doi:10.1103/PhysRevD.97.072001.

[96] M. Jiang, et al., Atmospheric Neutrino Oscillation Analysis with Improved Event Reconstruction in Super-Kamiokande IV, PTEP 2019 (5) (2019) 053F01. arXiv:1901.03230, doi:10.1093/ptep/ptz015 
[97] IceCube Oscillations: 3 years muon neutrino disappearance data, https: //icecube.wisc.edu/science/data/nu_osc (October 2016).

[98] M. Honda, M. Sajjad Athar, T. Kajita, K. Kasahara, S. Midorikawa, Atmospheric neutrino flux calculation using the NRLMSISE-00 atmospheric model, Phys. Rev. D92 (2) (2015) 023004. arXiv:1502.03916, doi:10.1103/PhysRevD.92.023004.

[99] M. Honda, Atmospheric neutrino flux updates, http://www.icrr. u-tokyo.ac.jp/ mhonda/ (Feb 2015).

[100] A. M. Dziewonski, D. L. Anderson, Preliminary reference earth model, Phys. Earth Planet. Interiors 25 (1981) 297-356. doi:10.1016/ 0031-9201(81) 90046-7.

[101] http://www-sk.icrr.u-tokyo.ac.jp/sk/publications/data/sk.atm.data.release.tar.gz.

[102] M. A. Acero, et al., New constraints on oscillation parameters from $\nu_{e}$ appearance and $\nu_{\mu}$ disappearance in the NOvA experiment, Phys. Rev. D98 (2018) 032012. arXiv:1806.00096, doi:10.1103/PhysRevD.98.032012

[103] K. Abe, et al., Updated T2K measurements of muon neutrino and antineutrino disappearance using $1.5 \times 10^{21}$ protons on target, Phys. Rev. D96 (1) (2017) 011102. arXiv:1704.06409, doi:10.1103/PhysRevD.96.011102.

[104] K. Abe, et al., Combined Analysis of Neutrino and Antineutrino Oscillations at T2K, Phys. Rev. Lett. 118 (15) (2017) 151801. arXiv:1701.00432, doi:10.1103/PhysRevLett.118.151801.

[105] M. Hartz, T2K NEUTRINO OSCILLATION RESULTS WITH DATA UP TO 2017 SUMMER, http://www.t2k.org/docs/talk/282 (August 2017).

[106] P. Adamson, et al., Combined analysis of $\nu_{\mu}$ disappearance and $\nu_{\mu} \rightarrow$ $\nu_{e}$ appearance in MINOS using accelerator and atmospheric neutrinos, Phys. Rev. Lett. 112 (2014) 191801. arXiv:1403.0867, doi:10.1103/ PhysRevLett.112.191801.

[107] M. H. Ahn, et al., Measurement of Neutrino Oscillation by the K2K Experiment, Phys. Rev. D74 (2006) 072003. arXiv:hep-ex/0606032, doi:10.1103/PhysRevD.74.072003. 
[108] P. Huber, M. Lindner, W. Winter, Simulation of long-baseline neutrino oscillation experiments with GLoBES (General Long Baseline Experiment Simulator), Comput. Phys. Commun. 167 (2005) 195. arXiv:hep-ph/ 0407333, doi:10.1016/j.cpc.2005.01.003

[109] P. Huber, J. Kopp, M. Lindner, M. Rolinec, W. Winter, New features in the simulation of neutrino oscillation experiments with GLoBES 3.0: General Long Baseline Experiment Simulator, Comput. Phys. Commun. 177 (2007) 432-438. arXiv:hep-ph/0701187, doi:10.1016/j.cpc.2007.05.004.

[110] P. Adamson, et al., Measurement of the neutrino mixing angle $\theta_{23}$ in NOvA, Phys. Rev. Lett. 118 (15) (2017) 151802. arXiv:1701.05891, doi:10. 1103/PhysRevLett.118.151802

[111] P. Adamson, et al., Constraints on Oscillation Parameters from $\nu_{e}$ Appearance and $\nu_{\mu}$ Disappearance in NOvA, Phys. Rev. Lett. 118 (23) (2017) 231801. arXiv:1703.03328, doi:10.1103/PhysRevLett.118.231801.

[112] A. Himmel, New neutrino oscillation results from NOVA, https://indico. cern.ch/event/696410/ (January 2018).

[113] T2K, neutrino beam flux prediction 2016, http://t2k-experiment.org/ result_category/flux/ (Oct 2015).

[114] M. Blennow, P. Coloma, E. Fernandez-Martinez, Reassessing the sensitivity to leptonic CP violation, JHEP 03 (2015) 005. arXiv:1407.3274, doi: 10.1007/JHEP03(2015)005.

[115] X. Qian, A. Tan, W. Wang, J. Ling, R. McKeown, C. Zhang, Statistical Evaluation of Experimental Determinations of Neutrino Mass Hierarchy, Phys. Rev. D 86 (2012) 113011. arXiv:1210.3651, doi:10.1103/ PhysRevD.86.113011.

[116] E. Ciuffoli, J. Evslin, X. Zhang, Confidence in a neutrino mass hierarchy determination, JHEP 01 (2014) 095. arXiv:1305.5150, doi:10.1007/ JHEP01(2014)095.

[117] M. Blennow, P. Coloma, P. Huber, T. Schwetz, Quantifying the sensitivity of oscillation experiments to the neutrino mass ordering, JHEP 03 (2014) 028. arXiv:1311.1822, doi:10.1007/JHEP03(2014)028

[118] M. Blennow, On the Bayesian approach to neutrino mass ordering, JHEP 01 (2014) 139. arXiv:1311.3183, doi:10.1007/JHEP01(2014)139 
[119] O. Vitells, A. Read, A comment on estimating sensitivity to neutrino mass hierarchy in neutrino experimentsarXiv:1311.4076.

[120] P. Huber, M. Lindner, T. Schwetz, W. Winter, First hint for CP violation in neutrino oscillations from upcoming superbeam and reactor experiments, JHEP 11 (2009) 044. arXiv:0907.1896, doi:10.1088/1126-6708/2009/ $11 / 044$.

[121] S. S. Chatterjee, P. Pasquini, J. W. F. Valle, Resolving the atmospheric octant by an improved measurement of the reactor angle, Phys. Rev. D96 (1) (2017) 011303. arXiv:1703.03435, doi:10.1103/PhysRevD.96.011303

[122] S. S. Chatterjee, P. Pasquini, J. W. F. Valle, Probing atmospheric mixing and leptonic CP violation in current and future long baseline oscillation experiments, Phys. Lett. B771 (2017) 524-531. arXiv:1702.03160, doi: $10.1016 / j$.physletb.2017.05.080

[123] I. Esteban, M. C. Gonzalez-Garcia, A. Hernandez-Cabezudo, M. Maltoni, T. Schwetz, Global analysis of three-flavour neutrino oscillations: synergies and tensions in the determination of $\theta_{23}, \delta_{C P}$, and the mass ordering, JHEP 01 (2019) 106. arXiv:1811.05487, doi:10.1007/JHEP01(2019)106.

[124] F. Capozzi, E. Lisi, A. Marrone, A. Palazzo, Current unknowns in the three neutrino framework, Prog. Part. Nucl. Phys. 102 (2018) 48-72. arXiv: 1804.09678, doi:10.1016/j.ppnp.2018.05.005.

[125] R. F. Streater, A. S. Wightman, PCT, spin and statistics, and all that, 1989.

[126] G. Barenboim, J. D. Lykken, A Model of CPT Violation for Neutrinos, Phys. Lett. B554 (2003) 73-80. arXiv:hep-ph/0210411, doi:10.1016/ S0370-2693(02) 03262-8

[127] G. Barenboim, J. Salvado, Cosmology and CPT violating neutrinos, Eur. Phys. J. C77 (11) (2017) 766. arXiv:1707.08155, doi:10.1140/epjc/ s10052-017-5347-y.

[128] S. Eliseev, et al., Direct Measurement of the Mass Difference of ${ }^{163} \mathrm{Ho}$ and ${ }^{163}$ Dy Solves the $Q$-Value Puzzle for the Neutrino Mass Determination, Phys. Rev. Lett. 115 (6) (2015) 062501. arXiv:1604.04210, doi:10.1103/ PhysRevLett.115.062501. 
[129] A. Giachero, et al., Measuring the electron neutrino mass with improved sensitivity: the HOLMES experiment, JINST 12 (02) (2017) C02046. arXiv:1612.03947, doi:10.1088/1748-0221/12/02/C02046.

[130] M. Aker, et al., Improved Upper Limit on the Neutrino Mass from a Direct Kinematic Method by KATRIN, Phys. Rev. Lett. 123 (22) (2019) 221802. arXiv:1909.06048, doi:10.1103/PhysRevLett.123.221802

[131] G. Barenboim, J. D. Lykken, MINOS and CPT-violating neutrinos, Phys. Rev. D80 (2009) 113008. arXiv:0908.2993, doi:10.1103/PhysRevD.80. 113008 .

[132] P. Adamson, et al., Measurement of Neutrino and Antineutrino Oscillations Using Beam and Atmospheric Data in MINOS, Phys. Rev. Lett. 110 (25) (2013) 251801. arXiv:1304.6335, doi:10.1103/PhysRevLett. 110.251801 .

[133] K. Abe, et al., Search for Differences in Oscillation Parameters for Atmospheric Neutrinos and Antineutrinos at Super-Kamiokande, Phys. Rev. Lett. 107 (2011) 241801. arXiv:1109.1621, doi:10.1103/PhysRevLett. 107.241801 .

[134] T. Ohlsson, S. Zhou, Extrinsic and Intrinsic CPT Asymmetries in Neutrino Oscillations, Nucl. Phys. B893 (2015) 482-500. arXiv:1408.4722, doi: 10.1016/j.nuclphysb.2015.02.015.

[135] M. G. Aartsen, et al., Measurement of Atmospheric Neutrino Oscillations at 6-56 GeV with IceCube DeepCore, Phys. Rev. Lett. 120 (7) (2018) 071801. arXiv:1707.07081, doi:10.1103/PhysRevLett.120.071801.

[136] A. Gando, et al., Constraints on $\theta_{13}$ from A Three-Flavor Oscillation Analysis of Reactor Antineutrinos at KamLAND, Phys. Rev. D83 (2011) 052002. arXiv:1009.4771, doi:10.1103/PhysRevD.83.052002

[137] Y. Abe, et al., Improved measurements of the neutrino mixing angle $\theta_{13}$ with the Double Chooz detector, JHEP 10 (2014) 086, [Erratum: JHEP02,074(2015)]. arXiv:1406.7763, doi:10.1007/JHEP02(2015) 074, 10.1007/JHEP10(2014)086.

[138] A. Himmel, First Oscillation Results with Neutrino and Antineutrino Beams in NOvA, http://nova-docdb.fnal.gov/cgi-bin/ ShowDocument?docid=30273 (June 2018). 
[139] K. Abe, et al., J-PARC Neutrino Beamline Upgrade Technical Design ReportarXiv: 1908.05141.

[140] R. Acciarri, et al., Long-Baseline Neutrino Facility (LBNF) and Deep Underground Neutrino Experiment (DUNE)arXiv:1512.06148.

[141] J. Strait, et al., Long-Baseline Neutrino Facility (LBNF) and Deep Underground Neutrino Experiment (DUNE)arXiv:1601.05823.

[142] R. Acciarri, et al., Long-Baseline Neutrino Facility (LBNF) and Deep Underground Neutrino Experiment (DUNE)arXiv:1601.02984.

[143] B. Abi, et al., Deep Underground Neutrino Experiment (DUNE), Far Detector Technical Design Report, Volume I Introduction to DUNEarXiv: 2002.02967.

[144] B. Abi, et al., Deep Underground Neutrino Experiment (DUNE), Far Detector Technical Design Report, Volume II DUNE PhysicsarXiv:2002.03005.

[145] B. Abi, et al., Deep Underground Neutrino Experiment (DUNE), Far Detector Technical Design Report, Volume III DUNE Far Detector Technical CoordinationarXiv:2002.03008.

[146] B. Abi, et al., Deep Underground Neutrino Experiment (DUNE), Far Detector Technical Design Report, Volume IV Far Detector Single-phase TechnologyarXiv:2002.03010.

[147] V. De Romeri, E. Fernandez-Martinez, M. Sorel, Neutrino oscillations at DUNE with improved energy reconstruction, JHEP 09 (2016) 030. arXiv: 1607.00293, doi:10.1007/JHEP09(2016)030.

[148] K. Abe, et al., Hyper-Kamiokande Design ReportarXiv:1805.04163.

[149] K. Abe, et al., Physics potentials with the second Hyper-Kamiokande detector in Korea, PTEP 2018 (6) (2018) 063C01. arXiv:1611.06118, doi:10.1093/ptep/pty044.

[150] P. Ballett, S. F. King, S. Pascoli, N. W. Prouse, T. Wang, Sensitivities and synergies of DUNE and T2HK, Phys. Rev. D96 (3) (2017) 033003. arXiv:1612.07275, doi:10.1103/PhysRevD.96.033003.

[151] F. Capozzi, S. W. Li, G. Zhu, J. F. Beacom, DUNE as the Next-Generation Solar Neutrino Experiment, Phys. Rev. Lett. 123 (13) (2019) 131803. arXiv:1808.08232, doi:10.1103/PhysRevLett.123.131803. 
[152] S. Adrian-Martinez, et al., Letter of intent for KM3NeT 2.0, J. Phys. G43 (8) (2016) 084001. arXiv:1601.07459, doi:10.1088/0954-3899/43/ $8 / 084001$.

[153] J. P. Yanez, A. Kouchner, Measurement of atmospheric neutrino oscillations with very large volume neutrino telescopes, Adv. High Energy Phys. 2015 (2015) 271968. arXiv:1509.08404, doi:10.1155/2015/271968

[154] M. Ribordy, A. Y. Smirnov, Improving the neutrino mass hierarchy identification with inelasticity measurement in PINGU and ORCA, Phys. Rev. D87 (11) (2013) 113007. arXiv:1303.0758, doi:10.1103/PhysRevD.87. 113007 .

[155] W. Winter, Atmospheric Neutrino Oscillations for Earth Tomography, Nucl. Phys. B908 (2016) 250-267. arXiv:1511.05154, doi:10.1016/j. nuclphysb.2016.03.033.

[156] M. G. Aartsen, et al., Letter of Intent: The Precision IceCube Next Generation Upgrade (PINGU)arXiv:1401.2046.

[157] F. Capozzi, E. Lisi, A. Marrone, PINGU and the neutrino mass hierarchy: Statistical and systematic aspects, Phys. Rev. D91 (2015) 073011. arXiv: 1503.01999, doi:10.1103/PhysRevD.91.073011.

[158] S. Ahmed, et al., Physics Potential of the ICAL detector at the Indiabased Neutrino Observatory (INO), Pramana 88 (5) (2017) 79. arXiv: 1505.07380, doi:10.1007/s12043-017-1373-4.

[159] A. Ghosh, T. Thakore, S. Choubey, Determining the Neutrino Mass Hierarchy with INO, T2K, NOvA and Reactor Experiments, JHEP 04 (2013) 009. arXiv:1212.1305, doi:10.1007/JHEP04(2013)009.

[160] S. T. Petcov, M. Piai, The LMA MSW solution of the solar neutrino problem, inverted neutrino mass hierarchy and reactor neutrino experiments, Phys. Lett. B533 (2002) 94-106. arXiv:hep-ph/0112074, doi: 10.1016/S0370-2693(02)01591-5.

[161] F. An, et al., Neutrino Physics with JUNO, J. Phys. G43 (3) (2016) 030401. arXiv:1507.05613, doi:10.1088/0954-3899/43/3/030401.

[162] Y. Farzan, M. Tortola, Neutrino oscillations and Non-Standard Interactions, Front.in Phys. 6 (2018) 10. arXiv:1710.09360, doi:10.3389/fphy. 2018.00010. 
[163] T. Alion, et al., Experiment Simulation Configurations Used in DUNE CDRarXiv:1606.09550.

[164] K. Abe, et al., Search for CP Violation in Neutrino and Antineutrino Oscillations by the T2K Experiment with $2.2 \times 10^{21}$ Protons on Target, Phys. Rev. Lett. 121 (17) (2018) 171802. arXiv:1807.07891, doi: 10.1103/PhysRevLett.121.171802.

[165] O. Peres, A. Smirnov, Atmospheric neutrinos: LMA oscillations, U(e3) induced interference and CP violation, Nucl. Phys. B 680 (2004) 479-509. arXiv:hep-ph/0309312, doi:10.1016/j.nuclphysb.2003.12.017.

[166] E. K. Akhmedov, M. Maltoni, A. Y. Smirnov, Neutrino oscillograms of the Earth: Effects of 1-2 mixing and CP-violation, JHEP 06 (2008) 072. arXiv:0804.1466, doi:10.1088/1126-6708/2008/06/072.

[167] O. L. Peres, A. Smirnov, Oscillations of very low energy atmospheric neutrinos, Phys. Rev. D 79 (2009) 113002. arXiv:0903.5323, doi:10.1103/ PhysRevD.79.113002.

[168] E. K. Akhmedov, S. Razzaque, A. Yu. Smirnov, Mass hierarchy, 2-3 mixing and CP-phase with Huge Atmospheric Neutrino Detectors, JHEP 02 (2013) 082, [Erratum: JHEP07,026(2013)]. arXiv:1205.7071, doi: 10.1007/JHEP02(2013)082,10.1007/JHEP07(2013)026.

[169] S. Razzaque, A. Yu. Smirnov, Super-PINGU for measurement of the leptonic CP-phase with atmospheric neutrinos, JHEP 05 (2015) 139. arXiv: 1406.1407, doi:10.1007/JHEP05(2015) 139.

[170] K. J. Kelly, P. A. Machado, I. Martinez Soler, S. J. Parke, Y. F. Perez Gonzalez, Sub-GeV Atmospheric Neutrinos and CP-Violation in DUNE, Phys. Rev. Lett. 123 (8) (2019) 081801. arXiv:1904.02751, doi:10.1103/ PhysRevLett.123.081801.

[171] M. C. Banuls, G. Barenboim, J. Bernabeu, Medium effects for terrestrial and atmospheric neutrino oscillations, Phys. Lett. B513 (2001) 391-400. arXiv:hep-ph/0102184, doi:10.1016/S0370-2693(01)00723-7.

[172] J. Bernabeu, S. Palomares-Ruiz, A. Perez, S. Petcov, The Earth mantle core effect in matter induced asymmetries for atmospheric neutrino oscillations, Phys. Lett. B 531 (2002) 90-98. arXiv:hep-ph/0110071, doi:10.1016/ S0370-2693(02) 01358-8. 
[173] J. Bernabeu, S. Palomares Ruiz, S. T. Petcov, Atmospheric neutrino oscillations, theta(13) and neutrino mass hierarchy, Nucl. Phys. B669 (2003) 255276. arXiv:hep-ph/0305152, doi:10.1016/j.nuclphysb.2003.07.025

[174] T. Tabarelli de Fatis, Prospects of measuring sin**2 2 Theta(13) and the sign of Delta $\mathrm{m}^{* * 2} 2$ with a massive magnetized detector for atmospheric neutrinos, Eur. Phys. J. C24 (2002) 43-50. arXiv:hep-ph/0202232, doi: $10.1007 / \mathrm{s} 100520200935$

[175] A. B. Sousa, First MINOS+ Data and New Results from MINOS, AIP Conf. Proc. 1666 (1) (2015) 110004. arXiv:1502.07715, doi:10.1063/1. 4915576.

[176] S. Palomares-Ruiz, S. T. Petcov, Three-neutrino oscillations of atmospheric neutrinos, theta(13), neutrino mass hierarchy and iron magnetized detectors, Nucl. Phys. B712 (2005) 392-410. arXiv:hep-ph/0406096, doi:10.1016/j.nuclphysb.2005.01.045

[177] D. Indumathi, M. V. N. Murthy, A Question of hierarchy: Matter effects with atmospheric neutrinos and anti-neutrinos, Phys. Rev. D71 (2005) 013001. arXiv:hep-ph/0407336, doi:10.1103/PhysRevD.71.013001.

[178] R. Gandhi, P. Ghoshal, S. Goswami, P. Mehta, S. Uma Sankar, Probing the nu mass hierarchy via atmospheric nu(mu) + anti-nu(mu) survival rates in megaton water Cerenkov detectorsarXiv:hep-ph/0506145

[179] S. T. Petcov, T. Schwetz, Determining the neutrino mass hierarchy with atmospheric neutrinos, Nucl. Phys. B740 (2006) 1-22. arXiv:hep-ph/ 0511277, doi:10.1016/j.nuclphysb.2006.01.020.

[180] P. Huber, M. Maltoni, T. Schwetz, Resolving parameter degeneracies in long-baseline experiments by atmospheric neutrino data, Phys. Rev. D71 (2005) 053006. arXiv:hep-ph/0501037, doi:10.1103/PhysRevD.71. 053006 .

[181] A. Samanta, The Mass hierarchy with atmospheric neutrinos at INO, Phys. Lett. B673 (2009) 37-46. arXiv:hep-ph/0610196, doi:10.1016/ j.physletb.2009.01.067

[182] R. Gandhi, P. Ghoshal, S. Goswami, P. Mehta, S. U. Sankar, S. Shalgar, Mass Hierarchy Determination via future Atmospheric Neutrino Detectors, Phys. Rev. D76 (2007) 073012. arXiv:0707.1723, doi:10.1103/ PhysRevD.76.073012 
[183] R. Gandhi, P. Ghoshal, S. Goswami, S. U. Sankar, Resolving the Mass Hierarchy with Atmospheric Neutrinos using a Liquid Argon Detector, Phys. Rev. D78 (2008) 073001. arXiv:0807.2759, doi:10.1103/PhysRevD.78. 073001 .

[184] A. Samanta, Discrimination of mass hierarchy with atmospheric neutrinos at a magnetized muon detector, Phys. Rev. D81 (2010) 037302. arXiv: 0907.3540, doi:10.1103/PhysRevD.81.037302.

[185] V. Barger, R. Gandhi, P. Ghoshal, S. Goswami, D. Marfatia, S. Prakash, S. K. Raut, S. U. Sankar, Neutrino mass hierarchy and octant determination with atmospheric neutrinos, Phys. Rev. Lett. 109 (2012) 091801. arXiv:1203.6012, doi:10.1103/PhysRevLett.109.091801.

[186] M. Blennow, T. Schwetz, Identifying the Neutrino mass Ordering with INO and NOvA, JHEP 08 (2012) 058, [Erratum: JHEP11,098(2012)]. arXiv: 1203.3388, doi:10.1007/JHEP08(2012) 058, 10.1007/JHEP11(2012) 098

[187] A. Ghosh, S. Choubey, Measuring the Mass Hierarchy with Muon and Hadron Events in Atmospheric Neutrino Experiments, JHEP 10 (2013) 174. arXiv:1306.1423, doi:10.1007/JHEP10(2013)174.

[188] M. M. Devi, T. Thakore, S. K. Agarwalla, A. Dighe, Enhancing sensitivity to neutrino parameters at INO combining muon and hadron information, JHEP 10 (2014) 189. arXiv:1406.3689, doi:10.1007/JHEP10(2014)189.

[189] O. Mena, I. Mocioiu, S. Razzaque, Neutrino mass hierarchy extraction using atmospheric neutrinos in ice, Phys. Rev. D78 (2008) 093003. arXiv:0803. 3044, doi:10.1103/PhysRevD .78.093003.

[190] M. Aartsen, et al., Development of an analysis to probe the neutrino mass ordering with atmospheric neutrinos using three years of IceCube DeepCore data, Eur. Phys. J. C 80 (1) (2020) 9. arXiv:1902.07771, doi:10.1140/ epjc/s10052-019-7555-0.

[191] F. Capozzi, E. Lisi, A. Marrone, Probing the neutrino mass ordering with KM3NeT-ORCA: Analysis and perspectives, J. Phys. G45 (2) (2018) 024003. arXiv:1708.03022, doi:10.1088/1361-6471/aa9503

[192] S.-F. Ge, K. Hagiwara, C. Rott, A Novel Approach to Study Atmospheric Neutrino Oscillation, JHEP 06 (2014) 150. arXiv:1309.3176, doi:10. 1007/JHEP06 (2014)150 
[193] S.-F. Ge, K. Hagiwara, Physics Reach of Atmospheric Neutrino Measurements at PINGU, JHEP 09 (2014) 024. arXiv:1312.0457, doi: 10.1007/JHEP09(2014)024.

[194] S. K. Agarwalla, T. Li, O. Mena, S. Palomares-Ruiz, Exploring the Earth matter effect with atmospheric neutrinos in icearXiv:1212.2238.

[195] D. Franco, C. Jollet, A. Kouchner, V. Kulikovskiy, A. Meregaglia, S. Perasso, T. Pradier, A. Tonazzo, V. Van Elewyck, Mass hierarchy discrimination with atmospheric neutrinos in large volume ice/water Cherenkov detectors, JHEP 04 (2013) 008. arXiv:1301.4332, doi:10. 1007/JHEP04(2013)008

[196] W. Winter, Neutrino mass hierarchy determination with IceCube-PINGU, Phys. Rev. D88 (1) (2013) 013013. arXiv:1305.5539, doi:10.1103/ PhysRevD.88.013013

[197] M. Blennow, T. Schwetz, Determination of the neutrino mass ordering by combining PINGU and Daya Bay II, JHEP 09 (2013) 089. arXiv:1306. 3988, doi:10.1007/JHEP09(2013) 089 .

[198] M. G. Aartsen, et al., PINGU Sensitivity to the Neutrino Mass Hierarchy, in: Cosmic Frontier Workshop: Snowmass 2013 Menlo Park, USA, March 6-8, 2013, 2013. arXiv:1306.5846

URL http://www.slac.stanford.edu/econf/C1307292/docs/ submittedArxivFiles/1306.5846.pdf

[199] J. LoSecco, Measuring the muon-neutrino to muon anti-neutrino ratio in a high statistics atmospheric neutrino experiment, Phys. Rev. D 59 (1999) 117302. arXiv:hep-ph/9806318, doi:10.1103/PhysRevD.59.117302.

[200] C. Adams, et al., The Long-Baseline Neutrino Experiment: Exploring Fundamental Symmetries of the Universe, in: Snowmass 2013: Workshop on Energy Frontier Seattle, USA, June 30-July 3, 2013, 2013. arXiv: 1307.7335 .

URL http://lss.fnal.gov/archive/2014/pub/fermilab-pub-14-022. pdf

[201] F. Cavanna, M. Kordosky, J. Raaf, B. Rebel, LArIAT: Liquid Argon In A TestbeamarXiv:1406.5560. 
[202] R. Acciarri, et al., Michel Electron Reconstruction Using Cosmic-Ray Data from the MicroBooNE LArTPC, JINST 12 (09) (2017) P09014. arXiv: 1704.02927, doi:10.1088/1748-0221/12/09/P09014.

[203] M. Sorel, Expected performance of an ideal liquid argon neutrino detector with enhanced sensitivity to scintillation light, JINST 9 (2014) P10002. arXiv:1405.0848, doi:10.1088/1748-0221/9/10/P10002.

[204] R. W. Huff, Decay rate of bound muons, Annals of Physics 16 (2) (1961) 288 - 317. doi:10.1016/0003-4916(61)90039-2.

[205] T. Suzuki, D. F. Measday, J. P. Roalsvig, Total Nuclear Capture Rates for Negative Muons, Phys. Rev. C35 (1987) 2212. doi:10.1103/PhysRevC. 35.2212 .

[206] M. Tanabashi, et al., Review of Particle Physics, Phys. Rev. D98 (3) (2018) 030001. doi:10.1103/PhysRevD.98.030001.

[207] A. V. Klinskikh, S. Brianson, V. B. Brudanin, V. G. Egorov, C. Petitjean, M. V. Shirchenko, Muon capture in ar. the muon lifetime and yields of cl isotopes, Bulletin of the Russian Academy of Sciences: Physics 72 (6) (2008) 735-736. doi:10.3103/S106287380806004X URL https://doi .org/10.3103/S106287380806004X

[208] W. M. Foreman, A Demonstration of Light-Augmented Calorimetry For Low-Energy Electrons in Liquid Argon., Ph.D. thesis, Chicago U. (2019). doi:10.2172/1495330

URL http://lss.fnal.gov/archive/thesis/2000/ fermilab-thesis-2019-01.pdf

[209] A. Bertin, A. Vitale, A. Placci, Nuclear Capture of Muons in Argon and Neon, Phys. Rev. A 7 (1973) 2214-2217. doi:10.1103/PhysRevA.7.2214.

[210] S. Amoruso, et al., Measurement of the mu decay spectrum with the ICARUS liquid argon TPC, Eur. Phys. J. C33 (2004) 233-241. arXiv: hep-ex/0311040, doi:10.1140/epjc/s2004-01597-7.

[211] R. Acciarri, et al., Construction and Assembly of the Wire Planes for the MicroBooNE Time Projection Chamber, JINST 12 (03) (2017) T03003. arXiv: 1609.06169, doi:10.1088/1748-0221/12/03/T03003. 
[212] G. D. Barr, T. K. Gaisser, P. Lipari, S. Robbins, T. Stanev, A Three dimensional calculation of atmospheric neutrinos, Phys. Rev. D70 (2004) 023006. arXiv:astro-ph/0403630, doi:10.1103/PhysRevD.70.023006

[213] M. Honda, T. Kajita, K. Kasahara, S. Midorikawa, T. Sanuki, Calculation of atmospheric neutrino flux using the interaction model calibrated with atmospheric muon data, Phys. Rev. D75 (2007) 043006. arXiv:astro-ph/ 0611418, doi:10.1103/PhysRevD.75.043006.

[214] M. Honda, T. Kajita, K. Kasahara, S. Midorikawa, Improvement of low energy atmospheric neutrino flux calculation using the JAM nuclear interaction model, Phys. Rev. D83 (2011) 123001. arXiv:1102.2688, doi: 10.1103/PhysRevD.83.123001.

[215] C. Andreopoulos, et al., The GENIE Neutrino Monte Carlo Generator, Nucl. Instrum. Meth. A614 (2010) 87-104. arXiv:0905.2517, doi:10. $1016 /$ j.nima.2009.12.009.

[216] I. F. Albuquerque, G. F. Smoot, Measuring atmospheric neutrino oscillations with neutrino telescopes, Phys. Rev. D 64 (2001) 053008. arXiv: hep-ph/0102078, doi:10.1103/PhysRevD.64.053008

[217] A. Friedland, S. W. Li, Understanding the energy resolution of liquid argon neutrino detectors, Phys. Rev. D99 (3) (2019) 036009. arXiv:1811.06159, doi:10.1103/PhysRevD.99.036009.

[218] M. Gonzalez-Garcia, M. Maltoni, T. Schwetz, Status of the CPT violating interpretations of the LSND signal, Phys. Rev. D 68 (2003) 053007. arXiv: hep-ph/0306226, doi:10.1103/PhysRevD.68.053007.

[219] G. Balieiro Gomes, D. V. Forero, M. M. Guzzo, P. C. De Holanda, R. L. N. Oliveira, Quantum Decoherence Effects in Neutrino Oscillations at DUNEarXiv:1805.09818.

[220] A. Capolupo, S. Giampaolo, G. Lambiase, Decoherence in neutrino oscillations, neutrino nature and CPT violation, Phys. Lett. B 792 (2019) 298-303. arXiv:1807.07823, doi:10.1016/j.physletb.2019.03.062.

[221] J. Carrasco, F. Díaz, A. Gago, Probing CPT breaking induced by quantum decoherence at DUNE, Phys. Rev. D 99 (7) (2019) 075022. arXiv:1811. 04982, doi:10.1103/PhysRevD.99.075022. 
[222] V. A. Kostelecky, M. Mewes, Lorentz and CPT violation in neutrinos, Phys. Rev. D69 (2004) 016005. arXiv:hep-ph/0309025, doi:10.1103/ PhysRevD.69.016005.

[223] J. S. Diaz, Correspondence between nonstandard interactions and CPT violation in neutrino oscillationsarXiv:1506.01936.

[224] S. Kumar Agarwalla, M. Masud, Can Lorentz Invariance Violation affect the Sensitivity of Deep Underground Neutrino Experiment?arXiv:1912. 13306 .

[225] J. W. F. Valle, Resonant Oscillations of Massless Neutrinos in Matter, Phys. Lett. B199 (1987) 432-436. doi:10.1016/0370-2693(87)90947-6.

[226] E. Roulet, MSW effect with flavor changing neutrino interactions, Phys. Rev. D44 (1991) 935-938. doi:10.1103/PhysRevD.44.R935.

[227] M. M. Guzzo, A. Masiero, S. T. Petcov, On the MSW effect with massless neutrinos and no mixing in the vacuum, Phys. Lett. B260 (1991) 154-160. doi:10.1016/0370-2693(91) 90984-X.

[228] J. Kopp, P. A. N. Machado, S. J. Parke, Interpretation of MINOS data in terms of non-standard neutrino interactions, Phys. Rev. D82 (2010) 113002. arXiv:1009.0014, doi:10.1103/PhysRevD.82.113002

[229] M. C. Gonzalez-Garcia, M. Maltoni, J. Salvado, Testing matter effects in propagation of atmospheric and long-baseline neutrinos, JHEP 05 (2011) 075. arXiv:1103.4365, doi:10.1007/JHEP05(2011)075.

[230] A. Esmaili, A. Yu. Smirnov, Probing Non-Standard Interaction of Neutrinos with IceCube and DeepCore, JHEP 06 (2013) 026. arXiv: 1304.1042, doi: 10.1007/JHEP06 (2013) 026

[231] J. Salvado, O. Mena, S. Palomares-Ruiz, N. Rius, Non-standard interactions with high-energy atmospheric neutrinos at IceCube, JHEP 01 (2017) 141. arXiv:1609.03450, doi:10.1007/JHEP01(2017)141.

[232] J. Kopp, M. Lindner, T. Ota, J. Sato, Non-standard neutrino interactions in reactor and superbeam experiments, Phys. Rev. D77 (2008) 013007. arXiv:0708.0152, doi:10.1103/PhysRevD.77.013007. 
[233] F. T. Avignone, III, S. R. Elliott, J. Engel, Double Beta Decay, Majorana Neutrinos, and Neutrino Mass, Rev. Mod. Phys. 80 (2008) 481-516. arXiv: 0708.1033, doi:10.1103/RevModPhys.80.481.

[234] F. F. Deppisch, M. Hirsch, H. Pas, Neutrinoless Double Beta Decay and Physics Beyond the Standard Model, J. Phys. G39 (2012) 124007. arXiv: 1208.0727, doi:10.1088/0954-3899/39/12/124007.

[235] J. W. F. Valle, Neutrinoless Double Beta Decay With Quasi Dirac Neutrinos, Phys. Rev. D27 (1983) 1672-1674. doi:10.1103/PhysRevD.27.1672.

[236] L. Wolfenstein, Different Varieties of Massive Dirac Neutrinos, Nucl. Phys. B186 (1981) 147-152. doi:10.1016/0550-3213(81)90096-1

[237] S. T. Petcov, On Pseudodirac Neutrinos, Neutrino Oscillations and Neutrinoless Double beta Decay, Phys. Lett. 110B (1982) 245-249. doi: 10.1016/0370-2693(82)91246-1.

[238] M. Doi, M. Kenmoku, T. Kotani, H. Nishiura, E. Takasugi, PSEUDODIRAC NEUTRINO, Prog. Theor. Phys. 70 (1983) 1331. doi:10.1143/ PTP.70.1331,

[239] S. M. Bilenky, B. Pontecorvo, Neutrino Oscillations With Large Oscillation Length in Spite of Large (Majorana) Neutrino Masses?, Sov. J. Nucl. Phys. 38 (1983) 248, [Yad. Fiz.38,415(1983)].

[240] S. M. Bilenky, S. T. Petcov, Massive Neutrinos and Neutrino Oscillations, Rev. Mod. Phys. 59 (1987) 671, [Erratum: Rev. Mod. Phys.60,575(1988)]. doi:10.1103/RevModPhys.59.671

[241] C. Giunti, C. W. Kim, U. W. Lee, Oscillations of pseudoDirac neutrinos and the solar neutrino problem, Phys. Rev. D46 (1992) 3034-3039. arXiv: hep-ph/9205214, doi:10.1103/PhysRevD.46.3034.

[242] G. Dutta, A. S. Joshipura, PseudoDirac neutrinos in seesaw model, Phys. Rev. D51 (1995) 3838-3842. arXiv:hep-ph/9405291, doi:10.1103/ PhysRevD.51.3838.

[243] A. S. Joshipura, S. D. Rindani, Phenomenology of pseudoDirac neutrinos, Phys. Lett. B494 (2000) 114-123. arXiv:hep-ph/0007334, doi:10.1016/ S0370-2693(00) 01148-5. 
[244] Y. Nir, PseudoDirac solar neutrinos, JHEP 06 (2000) 039. arXiv:hep-ph/ 0002168, doi:10.1088/1126-6708/2000/06/039.

[245] B. Brahmachari, S. Choubey, Viability of bimaximal solution of the Zee mass matrix, Phys. Lett. B531 (2002) 99-104. arXiv:hep-ph/0111133, doi:10.1016/S0370-2693(02)01366-7.

[246] P. H. Frampton, M. C. Oh, T. Yoshikawa, Zee model confronts SNO data, Phys. Rev. D65 (2002) 073014. arXiv:hep-ph/0110300, doi:10.1103/ PhysRevD.65.073014.

[247] X.-G. He, Is the Zee model neutrino mass matrix ruled out?, Eur. Phys. J. C34 (2004) 371-376. arXiv:hep-ph/0307172, doi:10.1140/epjc/ s2004-01669-8

[248] A. Geiser, PseudoDirac neutrinos as a potential complete solution to the neutrino oscillation puzzle, Phys. Lett. B444 (1999) 358. arXiv:hep-ph/ 9901433, doi:10.1016/S0370-2693(98)01419-1.

[249] W. Krolikowski, Option of three pseudoDirac neutrinos, Acta Phys. Polon. B31 (2000) 663-672. arXiv:hep-ph/9910308.

[250] K. R. S. Balaji, A. Kalliomaki, J. Maalampi, Revisiting pseudoDirac neutrinos, Phys. Lett. B524 (2002) 153-160. arXiv:hep-ph/0110314, doi:10.1016/S0370-2693(01)01356-9.

[251] E. Ma, P. Roy, Model of four light neutrinos prompted by all desiderata, Phys. Rev. D52 (1995) R4780-R4783. arXiv:hep-ph/9504342, doi:10. 1103/PhysRevD.52.R4780.

[252] S. Goswami, A. S. Joshipura, Neutrino anomalies and quasi Dirac neutrinos, Phys. Rev. D65 (2002) 073025. arXiv:hep-ph/0110272, doi:10.1103/ PhysRevD.65.073025.

[253] M. Cirelli, G. Marandella, A. Strumia, F. Vissani, Probing oscillations into sterile neutrinos with cosmology, astrophysics and experiments, Nucl. Phys. B708 (2005) 215-267. arXiv:hep-ph/0403158, doi:10.1016/j. nuclphysb.2004.11.056.

[254] A. de Gouvea, W.-C. Huang, J. Jenkins, Pseudo-Dirac Neutrinos in the New Standard Model, Phys. Rev. D80 (2009) 073007. arXiv:0906.1611, doi:10.1103/PhysRevD.80.073007. 
[255] J. F. Beacom, N. F. Bell, D. Hooper, J. G. Learned, S. Pakvasa, T. J. Weiler, PseudoDirac neutrinos: A Challenge for neutrino telescopes, Phys. Rev. Lett. 92 (2004) 011101. arXiv:hep-ph/0307151, doi:10.1103/ PhysRevLett.92.011101

[256] A. Esmaili, Pseudo-Dirac Neutrino Scenario: Cosmic Neutrinos at Neutrino Telescopes, Phys. Rev. D81 (2010) 013006. arXiv:0909.5410, doi:10. 1103/PhysRevD.81.013006.

[257] A. Esmaili, Y. Farzan, Implications of the Pseudo-Dirac Scenario for Ultra High Energy Neutrinos from GRBs, JCAP 1212 (2012) 014. arXiv:1208. 6012, doi:10.1088/1475-7516/2012/12/014

[258] A. S. Joshipura, S. Mohanty, S. Pakvasa, Pseudo-Dirac neutrinos via a mirror world and depletion of ultrahigh energy neutrinos, Phys. Rev. D89 (3) (2014) 033003. arXiv:1307.5712, doi:10.1103/PhysRevD.89.033003

[259] G. J. Stephenson, Jr., J. T. Goldman, B. H. J. McKellar, M. Garbutt, Large mixing from small: PseudoDirac neutrinos and the singular seesaw, Int. J. Mod. Phys. A20 (2005) 6373-6390. arXiv:hep-ph/0404015, doi: 10.1142/S0217751X05028466.

[260] K. L. McDonald, B. H. J. McKellar, The Type-II Singular See-Saw Mechanism, Int. J. Mod. Phys. A22 (2007) 2211-2222. arXiv:hep-ph/0401073, doi:10.1142/S0217751X07036567

[261] Y. H. Ahn, S. K. Kang, C. S. Kim, A Model for Pseudo-Dirac Neutrinos: Leptogenesis and Ultra-High Energy Neutrinos, JHEP 10 (2016) 092. arXiv:1602.05276, doi:10.1007/JHEP10(2016) 092.

[262] D. Chang, O. C. W. Kong, Pseudo-Dirac neutrinos, Phys. Lett. B477 (2000) 416-423. arXiv:hep-ph/9912268, doi:10.1016/S0370-2693(00) 00228-8.

[263] L. A. Sanchez, W. A. Ponce, R. Martinez, SU(3) (c) x SU(3) $(\ell) \times \mathrm{U}(1)$ $(X)$ as an E(6) subgroup, Phys. Rev. D64 (2001) 075013. arXiv:hep-ph/ 0103244, doi:10.1103/PhysRevD.64.075013.

[264] R. M. Fonseca, M. Hirsch, Lepton number violation in 331 models, Phys. Rev. D94 (11) (2016) 115003. arXiv:1607.06328, doi:10.1103/ PhysRevD.94.115003. 
[265] S. Abel, A. Dedes, K. Tamvakis, Naturally small Dirac neutrino masses in supergravity, Phys. Rev. D71 (2005) 033003. arXiv:hep-ph/0402287, doi:10.1103/PhysRevD.71.033003.

[266] C. Athanassopoulos, et al., Evidence for $\bar{\nu}_{\mu} \rightarrow \bar{\nu}_{e}$ oscillations from the LSND experiment at LAMPF, Phys. Rev. Lett. 77 (1996) 3082-3085. arXiv:nucl-ex/9605003, doi:10.1103/PhysRevLett.77.3082

[267] A. Aguilar-Arevalo, et al., Evidence for neutrino oscillations from the observation of anti-neutrino(electron) appearance in a anti-neutrino(muon) beam, Phys. Rev. D64 (2001) 112007. arXiv:hep-ex/0104049, doi: 10.1103/PhysRevD.64.112007.

[268] J. N. Abdurashitov, et al., Measurement of the response of a Ga solar neutrino experiment to neutrinos from an Ar-37 source, Phys. Rev. C73 (2006) 045805. arXiv:nucl-ex/0512041, doi:10.1103/PhysRevC.73.045805.

[269] M. Laveder, Unbound neutrino roadmaps, Nucl. Phys. Proc. Suppl. 168 (2007) 344-346. doi:10.1016/j.nuclphysbps .2007.02.037.

[270] C. Giunti, M. Laveder, Short-Baseline Active-Sterile Neutrino Oscillations?, Mod. Phys. Lett. A22 (2007) 2499-2509. arXiv:hep-ph/0610352, doi: 10.1142/S0217732307025455.

[271] G. Mention, M. Fechner, T. Lasserre, T. A. Mueller, D. Lhuillier, M. Cribier, A. Letourneau, The Reactor Antineutrino Anomaly, Phys. Rev. D83 (2011) 073006. arXiv:1101.2755, doi:10.1103/PhysRevD.83.073006.

[272] S. Gariazzo, C. Giunti, M. Laveder, Y. F. Li, Model-independent $\bar{\nu}_{e}$ shortbaseline oscillations from reactor spectral ratios, Phys. Lett. B782 (2018) 13-21. arXiv:1801.06467, doi:10.1016/j.physletb.2018.04.057.

[273] M. Dentler, A. Hernandez-Cabezudo, J. Kopp, M. Maltoni, T. Schwetz, Sterile neutrinos or flux uncertainties? - Status of the reactor antineutrino anomaly, JHEP 11 (2017) 099. arXiv:1709.04294, doi:10.1007/ JHEP11(2017)099.

[274] P. Adamson, et al., Search for active-sterile neutrino mixing using neutralcurrent interactions in NOvA, Phys. Rev. D96 (7) (2017) 072006. arXiv: 1706.04592, doi:10.1103/PhysRevD.96.072006. 
[275] M. G. Aartsen, et al., Search for sterile neutrino mixing using three years of IceCube DeepCore data, Phys. Rev. D95 (11) (2017) 112002. arXiv: 1702.05160, doi:10.1103/PhysRevD.95.112002.

[276] P. Adamson, et al., Limits on Active to Sterile Neutrino Oscillations from Disappearance Searches in the MINOS, Daya Bay, and Bugey-3 Experiments, Phys. Rev. Lett. 117 (15) (2016) 151801, [Addendum: Phys. Rev. Lett.117,no.20,209901(2016)]. arXiv:1607.01177, doi:10.1103/ PhysRevLett.117.151801,10.1103/PhysRevLett.117.209901.

[277] N. Agafonova, et al., Limits on muon-neutrino to tau-neutrino oscillations induced by a sterile neutrino state obtained by OPERA at the CNGS beam, JHEP 06 (2015) 069. arXiv: 1503.01876, doi:10.1007/JHEP06(2015) 069.

[278] K. Abe, et al., Limits on sterile neutrino mixing using atmospheric neutrinos in Super-Kamiokande, Phys. Rev. D91 (2015) 052019. arXiv:1410.2008, doi:10.1103/PhysRevD.91.052019.

[279] P. Adamson, et al., Search for Sterile Neutrinos Mixing with Muon Neutrinos in MINOS, Phys. Rev. Lett. 117 (15) (2016) 151803. arXiv: 1607.01176, doi:10.1103/PhysRevLett.117.151803.

[280] P. Adamson, et al., Search for sterile neutrinos in MINOS and MINOS+ using a two-detector fit, Phys. Rev. Lett. 122 (9) (2019) 091803. arXiv: 1710.06488, doi:10.1103/PhysRevLett.122.091803.

[281] A. Albert, et al., Measuring the atmospheric neutrino oscillation parameters and constraining the $3+1$ neutrino model with ten years of ANTARES data, JHEP 06 (2019) 113. arXiv:1812.08650, doi:10.1007/JHEP06 (2019) 113.

[282] K. Abe, et al., Search for light sterile neutrinos with the T2K far detector Super-Kamiokande at a baseline of $295 \mathrm{~km}$, Phys. Rev. D99 (7) (2019) 071103. arXiv:1902.06529, doi:10.1103/PhysRevD.99.071103.

[283] P. Adamson, et al., Improved Constraints on Sterile Neutrino Mixing from Disappearance Searches in the MINOS, MINOS+, Daya Bay, and Bugey-3 ExperimentsarXiv:2002.00301.

[284] S. Gariazzo, C. Giunti, M. Laveder, Y. F. Li, Updated Global 3+1 Analysis of Short-BaseLine Neutrino Oscillations, JHEP 06 (2017) 135. arXiv:1703. 00860, doi:10.1007/JHEP06(2017) 135 . 
[285] M. Dentler, A. Hernandez-Cabezudo, J. Kopp, P. A. N. Machado, M. Maltoni, I. Martinez-Soler, T. Schwetz, Updated Global Analysis of Neutrino Oscillations in the Presence of eV-Scale Sterile Neutrinos, JHEP 08 (2018) 010. arXiv:1803.10661, doi:10.1007/JHEP08(2018)010.

[286] A. Diaz, C. A. Arguelles, G. H. Collin, J. M. Conrad, M. H. Shaevitz, Where Are We With Light Sterile Neutrinos?arXiv:1906.00045.

[287] C. Giunti, T. Lasserre, eV-scale Sterile NeutrinosarXiv:1901.08330, doi: 10.1146/annurev-nucl-101918-023755.

[288] S. Boser, C. Buck, C. Giunti, J. Lesgourgues, L. Ludhova, S. Mertens, A. Schukraft, M. Wurm, Status of Light Sterile Neutrino SearchesarXiv: 1906.01739 doi:10.1016/j.ppnp.2019.103736.

[289] J. Schechter, J. W. F. Valle, Neutrino Masses in SU(2) x U(1) Theories, Phys. Rev. D22 (1980) 2227. doi:10.1103/PhysRevD.22.2227.

[290] G. Anamiati, R. M. Fonseca, M. Hirsch, Quasi Dirac neutrino oscillations, Phys. Rev. D97 (9) (2018) 095008. arXiv:1710.06249, doi: 10.1103/PhysRevD.97.095008

[291] A. De Gouvea, K. J. Kelly, G. V. Stenico, P. Pasquini, Physics with Beam Tau-Neutrino Appearance at DUNE, Phys. Rev. D100 (1) (2019) 016004. arXiv:1904.07265, doi:10.1103/PhysRevD.100.016004.

[292] A. Ghoshal, A. Giarnetti, D. Meloni, On the role of the $\nu_{\tau}$ appearance in DUNE in constraining standard neutrino physics and beyond, JHEP 12 (2019) 126. arXiv:1906.06212, doi:10.1007/JHEP12(2019) 126.

[293] F. J. Escrihuela, D. V. Forero, O. G. Miranda, M. Tortola, J. W. F. Valle, On the description of nonunitary neutrino mixing, Phys. Rev. D92 (5) (2015) 053009, [Erratum: Phys. Rev.D93,no.11,119905(2016)]. arXiv:1503.08879, doi:10.1103/PhysRevD.93.119905,10.1103/ PhysRevD.92.053009.

[294] M. Blennow, P. Coloma, E. Fernandez-Martinez, J. Hernandez-Garcia, J. Lopez-Pavon, Non-Unitarity, sterile neutrinos, and Non-Standard neutrino Interactions, JHEP 04 (2017) 153. arXiv:1609.08637, doi:10.1007/ JHEP04 (2017) 153. 
[295] Y. Chikashige, R. N. Mohapatra, R. D. Peccei, Are There Real Goldstone Bosons Associated with Broken Lepton Number?, Phys. Lett. 98B (1981) 265-268. doi:10.1016/0370-2693(81)90011-3.

[296] G. B. Gelmini, M. Roncadelli, Left-Handed Neutrino Mass Scale and Spontaneously Broken Lepton Number, Phys. Lett. 99B (1981) 411-415. doi:10.1016/0370-2693(81)90559-1.

[297] J. Schechter, J. W. F. Valle, Neutrino Decay and Spontaneous Violation of Lepton Number, Phys. Rev. D25 (1982) 774. doi:10.1103/PhysRevD. 25. 774 .

[298] G. B. Gelmini, J. W. F. Valle, Fast Invisible Neutrino Decays, Phys. Lett. 142B (1984) 181-187. doi:10.1016/0370-2693(84)91258-9

[299] M. C. Gonzalez-Garcia, J. W. F. Valle, Fast Decaying Neutrinos and Observable Flavor Violation in a New Class of Majoron Models, Phys. Lett. B216 (1989) 360-366. doi:10.1016/0370-2693(89)91131-3

[300] K. Eguchi, et al., A High sensitivity search for anti-nu(e)'s from the sun and other sources at KamLAND, Phys. Rev. Lett. 92 (2004) 071301. arXiv: hep-ex/0310047, doi:10.1103/PhysRevLett.92.071301.

[301] A. M. Gago, R. A. Gomes, A. L. G. Gomes, J. Jones-Perez, O. L. G. Peres, Visible neutrino decay in the light of appearance and disappearance long baseline experiments, JHEP 11 (2017) 022. arXiv:1705.03074, doi: 10.1007/JHEP11(2017)022.

[302] P. Coloma, O. L. G. Peres, Visible neutrino decay at DUNEarXiv:1705. 03599

[303] M. V. Ascencio-Sosa, A. M. Calatayud-Cadenillas, A. M. Gago, J. JonesPerez, Matter effects in neutrino visible decay at future long-baseline experiments, Eur. Phys. J. C78 (10) (2018) 809. arXiv:1805.03279, doi:10.1140/epjc/s10052-018-6276-0.

[304] Z. Moss, M. H. Moulai, C. A. Arguelles, J. M. Conrad, Exploring a nonminimal sterile neutrino model involving decay at IceCube, Phys. Rev. D97 (5) (2018) 055017. arXiv:1711.05921, doi:10.1103/PhysRevD .97.055017.

[305] G. Pagliaroli, A. Palladino, F. L. Villante, F. Vissani, Testing nonradiative neutrino decay scenarios with IceCube data, Phys. Rev. D92 (11) (2015) 113008. arXiv:1506.02624, doi:10.1103/PhysRevD.92.113008. 
[306] Y. P. Porto-Silva, S. Prakash, O. L. G. Peres, H. Nunokawa, H. Minakata, Constraining visible neutrino decay at KamLAND and JUNOarXiv:2002. 12134

[307] J. N. Bahcall, N. Cabibbo, A. Yahil, Are neutrinos stable particles?, Phys. Rev. Lett. 28 (1972) 316-318. doi:10.1103/PhysRevLett.28.316.

[308] A. Acker, S. Pakvasa, Solar neutrino decay, Phys. Lett. B320 (1994) 320322. arXiv:hep-ph/9310207, doi:10.1016/0370-2693(94)90663-7.

[309] Z. G. Berezhiani, G. Fiorentini, M. Moretti, A. Rossi, Fast neutrino decay and solar neutrino detectors, Z. Phys. C54 (1992) 581-586. doi:10.1007/ BF01559483.

[310] Z. G. Berezhiani, M. Moretti, A. Rossi, Matter induced neutrino decay and solar anti-neutrinos, Z. Phys. C58 (1993) 423-428. doi:10.1007/ BF01557699.

[311] A. Bandyopadhyay, S. Choubey, S. Goswami, MSW mediated neutrino decay and the solar neutrino problem, Phys. Rev. D63 (2001) 113019. arXiv:hep-ph/0101273, doi:10.1103/PhysRevD.63.113019.

[312] A. Bandyopadhyay, S. Choubey, S. Goswami, Neutrino decay confronts the SNO data, Phys. Lett. B555 (2003) 33-42. arXiv:hep-ph/0204173, doi:10.1016/S0370-2693(03)00044-3.

[313] R. Picoreti, M. M. Guzzo, P. C. de Holanda, O. L. G. Peres, Neutrino Decay and Solar Neutrino Seasonal Effect, Phys. Lett. B761 (2016) 70-73. arXiv:1506.08158, doi:10.1016/j.physletb.2016.08.007

[314] J. M. Berryman, A. de Gouvea, D. Hernandez, Solar Neutrinos and the Decaying Neutrino Hypothesis, Phys. Rev. D92 (7) (2015) 073003. arXiv: 1411.0308, doi:10.1103/PhysRevD.92.073003.

[315] G.-Y. Huang, S. Zhou, Constraining Neutrino Lifetimes and Magnetic Moments via Solar Neutrinos in the Large Xenon Detectors, JCAP 02 (2019) 024. arXiv:1810.03877, doi:10.1088/1475-7516/2019/02/024

[316] P. Lipari, M. Lusignoli, On exotic solutions of the atmospheric neutrino problem, Phys. Rev. D60 (1999) 013003. arXiv:hep-ph/9901350, doi: 10.1103/PhysRevD.60.013003. 
[317] M. C. Gonzalez-Garcia, M. Maltoni, Status of Oscillation plus Decay of Atmospheric and Long-Baseline Neutrinos, Phys. Lett. B663 (2008) 405409. arXiv:0802.3699, doi:10.1016/j.physletb.2008.04.041

[318] R. A. Gomes, A. L. G. Gomes, O. L. G. Peres, Constraints on neutrino decay lifetime using long-baseline charged and neutral current data, Phys. Lett. B740 (2015) 345-352. arXiv:1407.5640, doi:10.1016/j.physletb. 2014.12.014.

[319] S. Choubey, D. Dutta, D. Pramanik, Invisible neutrino decay in the light of NOvA and T2K data, JHEP 08 (2018) 141. arXiv:1805.01848, doi: 10.1007/JHEP08(2018) 141.

[320] P. B. Denton, I. Tamborra, Invisible Neutrino Decay Could Resolve IceCube's Track and Cascade Tension, Phys. Rev. Lett. 121 (12) (2018) 121802. arXiv:1805.05950, doi:10.1103/PhysRevLett.121.121802.

[321] M. D. Messier, Evidence for neutrino mass from observations of atmospheric neutrinos with Super-Kamiokande, Ph.D. thesis, Boston U. (1999). URL http://wwwlib.umi.com/dissertations/fullcit?p9923965

[322] E. A. Paschos, J. Y. Yu, Neutrino interactions in oscillation experiments, Phys. Rev. D65 (2002) 033002. arXiv:hep-ph/0107261, doi:10.1103/ PhysRevD.65.033002.

[323] S. Choubey, S. Goswami, D. Pramanik, A study of invisible neutrino decay at DUNE and its effects on $\theta_{23}$ measurement, JHEP 02 (2018) 055. arXiv: 1705.05820, doi:10.1007/JHEP02(2018) 055

[324] A. Ghoshal, A. Giarnetti, D. Meloni, Neutrino Invisible Decay at DUNE: a multi-channel analysisarXiv:2003.09012.

[325] T. Abrahao, H. Minakata, H. Nunokawa, A. A. Quiroga, Constraint on Neutrino Decay with Medium-Baseline Reactor Neutrino Oscillation Experiments, JHEP 11 (2015) 001. arXiv:1506.02314, doi:10.1007/ JHEP11(2015)001.

[326] S. Choubey, S. Goswami, C. Gupta, S. M. Lakshmi, T. Thakore, Sensitivity to neutrino decay with atmospheric neutrinos at the INO-ICAL detector, Phys. Rev. D97 (3) (2018) 033005. arXiv:1709.10376, doi: 10.1103/PhysRevD.97.033005. 
[327] S. H. Seo, et al., Spectral Measurement of the Electron Antineutrino Oscillation Amplitude and Frequency using 500 Live Days of RENO Data, Phys. Rev. D98 (1) (2018) 012002. arXiv:1610.04326, doi:10.1103/PhysRevD. 98.012002

[328] R. Wendell, et al., Atmospheric neutrino oscillation analysis with subleading effects in Super-Kamiokande I, II, and III, Phys. Rev. D81 (2010) 092004. arXiv:1002.3471, doi:10.1103/PhysRevD.81.092004.

[329] M. G. Aartsen, et al., Measurement of Atmospheric Tau Neutrino Appearance with IceCube DeepCore, Phys. Rev. D99 (3) (2019) 032007. arXiv:1901.05366, doi:10.1103/PhysRevD.99.032007.

[330] Three-year high-statistics neutrino oscillation samples, https://icecube. wisc.edu/science/data/highstats_nuosc_3y (2019).

[331] K. Abe, et al., Search for Electron Antineutrino Appearance in a Longbaseline Muon Antineutrino Beam, Phys. Rev. Lett. 124 (16) (2020) 161802. arXiv:1911.07283, doi:10.1103/PhysRevLett.124.161802.

[332] K. Abe, et al., Constraint on the matter-antimatter symmetry-violating phase in neutrino oscillations, Nature 580 (7803) (2020) 339-344. arXiv: 1910.03887, doi:10.1038/s41586-020-2177-0.

[333] M. A. Acero, et al., First Measurement of Neutrino Oscillation Parameters using Neutrinos and Antineutrinos by NOvA, Phys. Rev. Lett. 123 (15) (2019) 151803. arXiv:1906.04907, doi:10.1103/PhysRevLett. 123.151803 . 